\title{
Local myocardial adaptations during chronic ventricular pacing
}

Citation for published version (APA):

Oosterhout, M. F. M. (1999). Local myocardial adaptations during chronic ventricular pacing. [Doctoral Thesis, Maastricht University]. Universiteit Maastricht. https://doi.org/10.26481/dis.19990528mo

Document status and date:

Published: 01/01/1999

DOI:

10.26481/dis.19990528mo

Document Version:

Publisher's PDF, also known as Version of record

\section{Please check the document version of this publication:}

- A submitted manuscript is the version of the article upon submission and before peer-review. There can be important differences between the submitted version and the official published version of record.

People interested in the research are advised to contact the author for the final version of the publication, or visit the DOI to the publisher's website.

- The final author version and the galley proof are versions of the publication after peer review.

- The final published version features the final layout of the paper including the volume, issue and page numbers.

Link to publication

\footnotetext{
General rights rights.

- You may freely distribute the URL identifying the publication in the public portal. please follow below link for the End User Agreement:

www.umlib.nl/taverne-license

Take down policy

If you believe that this document breaches copyright please contact us at:

repository@maastrichtuniversity.nl

providing details and we will investigate your claim.
}

Copyright and moral rights for the publications made accessible in the public portal are retained by the authors and/or other copyright owners and it is a condition of accessing publications that users recognise and abide by the legal requirements associated with these

- Users may download and print one copy of any publication from the public portal for the purpose of private study or research.

- You may not further distribute the material or use it for any profit-making activity or commercial gain

If the publication is distributed under the terms of Article $25 \mathrm{fa}$ of the Dutch Copyright Act, indicated by the "Taverne" license above, 


\title{
LOCAL MYOCARDIAL ADAPTATIONS DURING CHRONIC VENTRICULAR PACING
}

\author{
PROEFSCHRIFT
}

Ter verkrijging van de graad van doctor aan

de Universiteit Maastricht,

op gezag van de Rector Magnificus,

Prof.dr. A.C. Nieuwenhuijzen Kruseman,

volgens het besluit van het College van Decanen,

in het openbaar te verdedigen

op vrijdag 28 mei 1999 om 16.00 uur

door

Mattheus Franciscus Maria van Oosterhout

geboren te Amsterdam op 23 juni 1966 
Promotores:

Prof.dr. R.S. Reneman

Prof.dr.ir. T. Arts

Co-Promotor:

Dr. F.W. Prinzen

Beoordelingscommissie:

Prof.dr. G.J. van der Vusse (voorzitter)

Prof.dr. M.J.A.P. Daemen

Prof.dr. L. Kappenberger (Cantre Hospitalier Universitaire Vaudois, Lausanne, Zwitserland)

Prof.dr. H.J.J. Wellens

Prof.dr. N. Westerhof

This thesis comprises studies carried out in the department of Physiology of the Cardiovascular Research Institute Maastricht (CARIM), University Maastricht, Maastricht, The Netherlands.

The studies described were supported by a grant of Bakken Research Center, Medtronic.

Financial support by the Netherlands Heart Foundation and the Stichting Hartsvrienden Rescar

for the publication of this thesis is greatfully acknowledged.

We thank Bakken Research Center, Heart Failure Management and Perkin-Elmer for their additional support. 


\section{Chapter 1 Introduction}

Chapter 2 Literature review

Chapter 3 Fluorescent microspheres to measure organ perfusion:

validation of a simplified sample processing technique

Chapter 4 Fluorescent microspheres are superior to radioactive microspheres in chronic blood flow measurements

Chapter 5 Asynchronous electrical activation induces asymmetrical hypertrophy of the left ventricular wall

Chapter 6 Regional blood flow and activity of metabolic enzymes in canine hearts with pacing induced asymmetric hypertrophy

Chapter 7 Ventricular pacing induces remodeling in hypertrophying dog hearts

Chapter 8 Optimizing of ventricular function by improving the activation sequence during ventricular pacing

Chapter 9 General Discussion

Chapter 10 Summary

Samenvatting

Curriculum vitae

Publications

Dankwoord

\section{CONTENTS} 1 
GENERAL INTRODUCTION 
$\mathrm{O}$ ptimal cardiac function depends on the coordination of contraction of all ventricular myocytes. This coordination is mediated by the conduction of the impulse through the rapid conduction pathways (the His bundle and the Purkinje fibers) within the ventricular myocardium. In healthy human hearts all myocardial cells are depolarized within $80-100 \mathrm{~ms}$ : In patients with a defective conduction system, as in left bundle branch block, or in disturbed conduction, as in ventricular pacing, the time ror tota' utprobicantion ima' incease sigmificant. ly. Already in 1925 it was shown that abnormal asynchronous electrical activation has a great impact on the pattern and extent of regional wall motion, and on global pump function. More recently it was shown that in short term experiments ventricular pacing reduces global pump function. ${ }^{24}$ Asynchronous electrical activation has also important effects on regional myocardial function. In previous studies it was shown that ventricular pacing decreases fiber shortening, contractile work, myocardial blood flow and oxygen consumption in early activated regions and increases these parameters in late activated. regions. ${ }^{6.8}$

The ventricular wall is known to adapt to global changes in workload by changing cardiomyocyte size and extracellular matrix composition. The major question addressed in this thesis is whether a long lasting condition of local differenies in workload between various regions in the left ventricle leads to locally different degrees of myocardial grovth (hypertrophy).

Changes in cardiomyocyte size and extracellular matrix are supposed to be regulated by neurohumoral factors ${ }^{910}$ and crdiac load. ${ }^{112}$ The exact contribution of neurohumoral fictors, like angiotensin $\mathrm{Il}$, alpha-1 agonists and thumsin so hypertrophy in vivo, however, is not clear, because their growth promoting action may be confounded by their hemodynamic effects. Alternatively, local cellular loading may induce growth at the level of individual myocytes. Mathematical simulations ${ }^{13}$ and experimental studies on unloaded papillary muscles ${ }^{\text {It }}$ and isolated myocytes ${ }^{12}$ support the role of locally load regulated growth.

Whatever the true stimuli are, it is well-known that different loading conditions result in different kinds of hypertrophy, both at the organ and at the (sub)cellular level. In pressure overload, as in hypertension, aortic valve stenosis and aortic coarctation, concentric hypertrophy will develop. In concentric hypertrophy the LV wall thickness increases with an unaltered LV chamber 
volume. In volume overload, as in aortic and mitral valve insufficiency, endurance training and atrial septum defects, eccentric hypertrophy develops. In eccentric hypertrophy LV cavity volume increases with a proportional increase in LV wall thickness. Also, in such genetic disorders as hypertrophic obstructive cardiomyopathy (HOCM) hypertrophy may develop. Depending on the etiologic factor, hypertrophy of the ventricle is associated with increased myocyte volume (change in myocyte length, width or both), a change in the composition of the extracellular matrix (collagen) and/or a change in capillarization. In hypertrophy changes in cellular metabolism, electrophysiology and mechanical properties have been shown to occur. In chapter 2 the relevant literature concerning these aspects will be reviewed in detail.

\section{RATIONALE}

The aim of this thesis is to study the adaptation of the myocardium in chronic ventricular pacing. The major hypothesis to be tested is that in the in situ heart asynchronous electrical activation leads to asymmetric hypertrophy due to an inhomogeneous distribution of workload. This hypothesis was tested in normal hearts and hearts hypertrophying due to pressure overload. Furthermore, it is investigated whether local differences in the degree of hypertrophy are associated with local differences in myocardial blood flow and in metabolic enzyme activity. The effect of chronic pacing on global pump function was investigated as well. Finally, it was investigated whether in pacing hemodynamic function can be optimized by changing and/or combining pacing sites.

The clinical rationale of this study is to obtain better insight into the etiology of myocardial adaptation in asynchronous electrical activation. Currently, worldwide approximately 400.000 ventricular pacemakers are implanted yearly. Conduction abnormalities, like left bundle branch block, are present in $0.02-0.3 \%$ of the general population, the prevalence increases with age. Nevertheless, the consequences of long term asynchronous electrical activation for myocardial function and structure are not well-known. Systematic studies on the structural changes during chronic asynchronous activation have hardly been performed. Karpawich et al. ${ }^{14}$ showed fiber disarray and other histologic abnormalities in chronically paced puppies. These investigators also 
showed that these derangements could be prevented when the hearts were paced from the high ventricular septum instead of the conventionally used right ventricular apex, In high septal pacing the absence of histologic abnormalities was associated with more synchronous electrical activation and contraction patterns."

\section{OVERVIEW OF THE THESIS}

Because the occurence of hypertrophy was anticipated and myocardial hypertrophy is often associated with myocardial underperfusion leading to myocardial infarction, first a method was developed to measure blood flow in chronic animal studies. Microspheres labeled with radioactive isotopes are considered to be the 'gold standard' for blood flow measurements in the experimental setting. The use of radioactivity, however, has serious drawbacks, especially in chronic experiments: special animal housing is needed and, more importantly, with longer duration radioactivity of the isotopes may decay to undetectable levels. Recently, the use of microspheres labeled with fluorescent dyes were introduced as an alternative by Glenny et al..$^{\text {th }}$ We further improved this technique and in chapter 3 a novel method for tissue digestion and microsphere recovery, the sedimentation method, is described and validated for the use in normal and ischemic myocardium and various other organs in dogs. In chapter 4 the fluorescent microsphere technique is validated for the use in chronic experiments.

Next, in normal hearts we studied the effects of chronic asynchronous activation, as induced by left ventricular (LV) pacing, on regional geometry and microscopic structure of the LV wall and on global ventricular geometry and performance (chapter 5). Healthy, adult dogs were paced from the LV free wall for six months. LV geometry was studied by means of 2D echocardiography and LV function by means of pressure-volume analysis. Post mortem the hearts were analyzed histologically.

Having established that ventricular pacing leads to asymmetric hypertrophy, it was investigated whether local differences in the degree of hypertrophy within the same heart were associated with local differences in myocardial perfusion and the activity of various metabolic enzymes (chapter 6).

Subsequently, we investigated whether ventricular 
pacing can also induce local differences in growth and microscopic structure and in global ventricular geometry and performance in hypertrophic hearts. In this study, LV hypertrophy was induced by coarctation of the ascending aorta in puppies (chapter 7). During the progression of hypertrophy these animals were paced from the (clinically frequently used) RV apex. Local myocardial geometry was studied with the use of 2D- echocardiographic and X-ray marker analysis.

Finally, chapter 8 describes a short-term study in dogs on the hemodynamic effects of pacing. It was investigated whether hemodynamic improvements can be obtained by using other pacing sites than the conventionally used RV apex. Also the hemodynamic effect of multisite pacing was studied.

The thesis starts with a survey of the literature (chapter 2) and is completed with a general discussion (chapter 9). In the latter chapter, the findings of these studies are discussed with special emphasis on the clinical implications.

\section{LITERATURE}

1. Durrer D, Dam v. RT. Freud GE, lanse MT. Meyler H , Arzbaecher RC. Total excitation of the isolated human heart. Circulation. 1970:X1.1.899.912

2. Wiggers CJ. The muscular neactions of the mammalian ventricles to artificial surface stimuli. Am I Physiol. 1925:73:345.378.

3. Burkhotf D, Oikawa RY, Sagawa K. Influence of pacing site on canine left ventricular contraction. Am J Physiol. 1986;251:H428-H435

4. Little WC. Park RC. Freeman GL Effects of regional ischemia and ventricular pacing on LV dP/dtmax-end-diastolic volume relation. Am I Physiol $1087: 252: \mathrm{H} 933-\mathrm{H} 940$

5. Rosenquist M, Isaay K, Botvinick EH, Dae MW, Cockrell I, Abbott JA, Schiller NIS, Griffin IC. Relative importance of activation sequence compared to atrioventricular synchrony in left ventricular function. Am I Cardiol 1991;67:148. 156.

6. Prinzen FW, Augustijn CH, Allessie MA, Arts T, Delhaas T, Reneman RS. The time sequence of electrical and mechanical activation during spontaneous beating and ectopic stimulation. Eur Heart ]. 1992:13:535-543.

7. Prinzen FW, Augustijn CH, Arts T. Allessie MA, Reneman RS. Redistribution of myocardial fiber strain and blood flow by asynchronous activation. Am I Physiol. 1990:259: $\mathrm{H} 300-\mathrm{H} 308$.

8. Delhaas T, Arts T, Prinzen FW, Reneman RS. Regional fibre stress-fibre strain area as an estimate of regional blood. flow and oxygen demand in the canine heart | Physiol (London), 1994:477,3:481-496

9. Dzau VI. Tissue renin-angiotensin system in myocardial hypertrophy and failure Arch Int Med. 1993;153:937-942.

10. Long CS, Kariva K. Karns L, Simpson PC. Trophic factors for cardiac myocytes. i Hypertension. 199():8 (suppl. 7):\$219-5223

11. Cooper IV G. Cardiocyte adaptation to chronically altered load. Ann Rev Physiol 1987:49:501-518.

12. Sadoshima I. Izumo S. The cellular and molecular nesponse of cardiac myocytes to mechanical stress. Annu Rev Physiol 1997:59:551-57]

13. Arts T, Prinzen FW, Snoeckx LHEH, Rijcken IM Reneman RS. Adaptation of cardiac structure by mechanical feedback in the environment of the cell: A model study. Biophysical ]. 1994:66:953-961

14. Karpawich PP. Justice CD, Cavitt DL, Chang C-H. Developmental sequelae of fixed-rate ventricular pacing in the immature canine heart: An electrophysiologic. hemodynamic and histopathologic evaluation. Am Heart I. 1990;119:1077-1083.

15. Karpawich PP, Justice CD, Chang C-H, Gause CY, Kuhns I.R. Septal ventrieular pacing in the immature canine heart: A new perspective. Am Heart J. 1991;121:827 833.

16.Glenny RW. Bernand S, Brinkley M. Validation of fluorescent-labeled microspheres for measurement of regional organ perfusion. I Appl Physiol. 1993;74:2585-2597. 



\section{LITERATURE}

\section{REVIEW}




\section{VENTRICULAR PACING}

\section{Normal electrical impulse conduction}

During normal heart beats rhythmical electrical impulses are generated in the sino-atrial (SA) node, which is located in the right atrial wall near the entrance of the superior caval vein. The action potential spreads from the SA node over the atria to the atrio-ventricular (AV) node, which is located on the low right side of the atrial septum (Fig. 2.1). In man the AV node delays the electrical transmission by about $80 \mathrm{~ms}$. In a standard electrocardiogram (ECG) the time from impulse formation in the SA node to the begining of ventricular activation is reflected by the $\mathrm{PQ}$ interval. The electrical impulse is subsequently transmitted from the atria to the ventricles through the bundle of His. The bundle of His is a bundle of Purkinje fibers that passes through the atrioventricular barrier and

Figure 2.1 Sequence of cardiac excitation: normal and pacing

A: Atrial activation sequence during simus rhytim; P-tone of the ECG. Ventricular actioation during simus rhythm (B), RV apex pacing (C) and LV free wall pacing (D). The signals below the various panels depict the concomitant QRS complex of the ECG.

Lines denote $20 \mathrm{~ms}$ ischrone lines of the depolarization, carliest actioation occurs in black negions and latest activation in white regions.

$\mathrm{SCV}=$ superior caval vein. divides into the right and left bundle branches, from where the Purkinje fibers spread over the endocardium of both ventricles. By way of this specialized conduction tissue the ventricles are depolarized almost simultaneously. In isolated human hearts it was found that the anterior fasciculus of the left bundle activates the anterolateral area of the left ventricle (LV), whereas the posterior
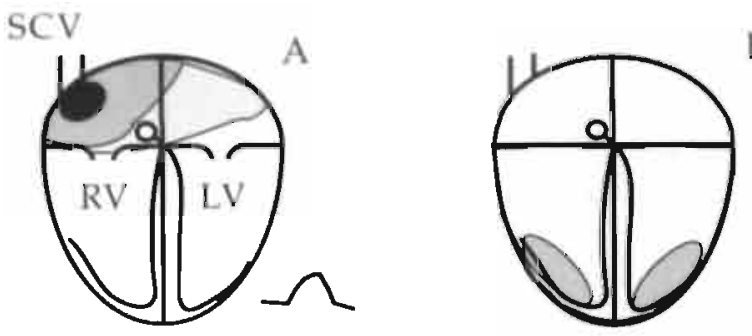

B

Sinus rhythm
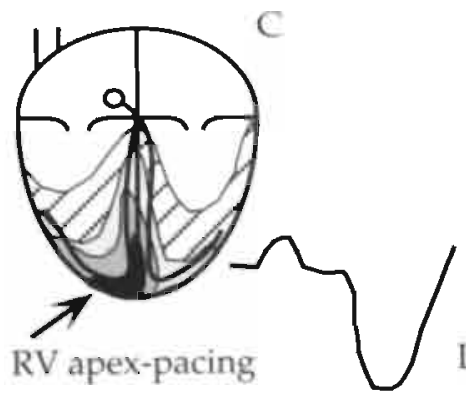

D
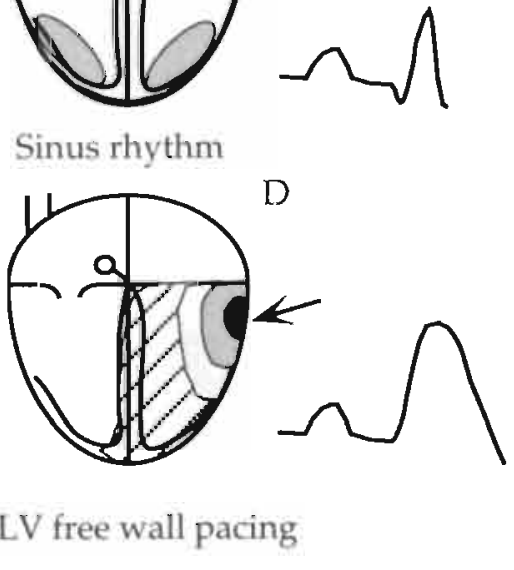
fasciculus activates the postero-inferior area of the LV. The posterobasal area is last activated. Total activation of the ventricles takes 62 to $80 \mathrm{~ms}$, corresponding with a QRS

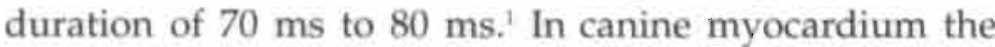
ventricles are activated within 35 to $40 \mathrm{~ms}^{2}$

\section{Abnormal electrical impulse conduction}

A malfunction at any level in the activation sequence may lead to distinctive disturbances in cardiac rhythm and/or coordination of contraction. Clinically the major problems in impulse formation and propagation are located at the level of the sinus node, the AV node, bundle of His, bundle branches and intraventricular purkinje system. Malfunction of the sinus node, induced by disturbances in impulse formation or impulse conduction, may lead to bradyarrhytmias and subsequently to syncope. A complete heart block (third degree AV block) leads to uncoupling of atrial and ventricular activation and resultins in uncoupling of the atrial and ventricular contraction sequence. This blockade is recognized on the ECG by $\mathrm{P}$ waves occuring independently from the QRS complex. Usually, in this condition the atrial rhythm is normal. Ventricular pacemaking is taken over by cardiomyocytes distal from the blockade. The resulting ventricular rhythm is much slower than the sinus rhythm. This abnormality often leads to ventricular hypertrophy and a reduction of cardiac pump function. The most common therapy for both sick sinus disease and complete heart block is implantation of a pacemaker.

Prolongation of ventricular activation can be observed in diseases affecting the ventricular conduction system. For example, blockade of the left or right bundle branch (LBBB, RBBB) markedly prolongs ventricular activatio. Asynchronous activation is also observed during ventricular extra systoles. This occurs because with an ectopic endogenous pacemaker in the ventricle, the electrical impulse largely bypasses the Purkinje system through much slower myocardial cell to cell conduction. Another cause of asynchronous electrical activation is pre-excitation of a myocardial region due to an accessory pathway bypassing the AV node, as is the case in the Wolff-Parkinson-White syndrome. Moreover, and quite relevant to this thesis, ventricular pacing with an exogenous pacemaker causes asynchronous electrical activation of the ventricles.

\section{Asynchronous electrical activation by ventricular pacing}

During epicardial ventricular pacing the propagation of the electrical impulse initially occurs by myocyte-to- 
myocyte condution, as opposed to normal ventricular activation over the intraventricular conduction system. Therefore, initially the electrical impulse propagates relatively slowly $(0.5-1 \mathrm{~m} / \mathrm{sec}$, Fig. 2.1). Presumably, at a particular site the impulse wave, conducted by the myocytes, enters the rapidly conducting $(2-4 \mathrm{~m} / \mathrm{sec})$ Purkinje system. The site of pacing (endocardial vs epicardial) determines the moment at which the impulse wave enters the Purkinje system. Due to these time consuming conduction phenomena ventricular pacing leads to an increase of the QRS duration in canine hearts from $35-40 \mathrm{~ms}$ during sinus rhythm to up to $150 \mathrm{~ms}$ during ventricular pacing. ${ }^{24}$ The direction and speed of myocardial activation depends on the pacing site. $^{2}$ In patients the most commonly used site for permanent cardiac pacing is the right ventricular (RV) apex. This location provides good fixation and a low stimulation threshold. RV apex pacing in man increases QRS duration to about $180 \mathrm{~ms}$ as compared to $100 \mathrm{~ms}$ during sinus rhythm. In left bundle branch block (LBBB) and in RV apex pacing the septum is activated relatively early. ${ }^{2.7}$ Therefore RV apex pacing is sometimes used as "experimental LBBB".

\section{Pacing modes}

Two major kinds of pacing can be recognized: single chamber pacing of either the atrium or the ventricle and synchronized pacing of atrium and ventricle. This AV synchronized pacing can be performed by pacing the ventricles a certain time interval after the moment of sensing the atrial activation or after the moment of pacing the atria. Pacemakers are categorized with a basic three-letter identification code according to the site of the pacing electrodes and the mode of pacing (see Table 2.1 for coding): $\mathrm{A}=$ atrium, $\mathrm{V}=$ ventricle, $\mathrm{D}=\mathrm{dual}(\mathrm{A}$ and $\mathrm{V}$ ). The first

\begin{tabular}{|c|c|c|c|}
\hline $\begin{array}{l}\text { Position } \\
\text { Category }\end{array}$ & $\begin{array}{c}\text { I } \\
\text { Chamber(s) } \\
\text { Paced }\end{array}$ & $\begin{array}{c}\text { Ii } \\
\text { Chamber(s) } \\
\text { Sensed }\end{array}$ & $\begin{array}{l}\text { III } \\
\text { Response } \\
\text { to sensing }\end{array}$ \\
\hline Letters & $\begin{array}{l}0=\text { none } \\
A=\text { atrium } \\
V=\text { Ventricle } \\
D=\text { dual }(A+V)\end{array}$ & $\begin{array}{l}0=\text { none } \\
A=\text { atrium } \\
V=\text { Ventricie } \\
D=\text { dual }(A+V)\end{array}$ & $\begin{array}{l}0=\text { none } \\
T=\text { triggered } \\
I=\text { inhibited } \\
D=\text { dual }(A+V)\end{array}$ \\
\hline
\end{tabular}

Table 2.1 The NASPE/BPEG generic Pacemaker coding

Positions I to III are exclusively used for antibradyarrhytmia function. Positions IV and V are used in some pacemakers and denote programmability and antitachyarrhytmia, respectively, and are not further discussed here. NASPE $=$ North American Socyity of Pacing and Electroplysiology, $B P E G=$ British Pacing and Electrophysiology Group (adapted from (122)). 
position denotes the chamber paced, the second position indicates the chamber sensed and the third position indicates the response to sensing, with $\mathrm{O}$ indicating not inhibited by spontaneous ventricular activation, I indicating inhibited response, $\mathrm{T}$ triggered response and $\mathrm{D}$ indicating the combination of $\mathrm{I}$ and $\mathrm{T}$ response. For example during VVI pacing, a single chamber (ventricle) will be paced only if there is no spontaneous ventricular activity sensed. During DDD pacing both the atrium and ventricle will be paced if no spontaneous activity is sensed. In case of spontaneous atrial rhythm and heart block, the atrial activity is sensed and triggers the pacemaker to pace the ventricle after a preset interval.

\section{Hemodynamic effects of ventricular pacing}

Ventricular pump function during pacing is modified by two factors: the AV-interval and the site of ventricular pacing. With ventricular single chamber pacing (VVI, VOO) the contraction of atria and ventricles is uncoupled, leading to the loss of the atrial contribution to LV filling. In patients cardiac output decreased by about $20 \%$ when decreasing the AV interval from $100-130$ to $0 \mathrm{~ms}^{10.11}$ Others found a decrease in cardiac output of $4-16 \% \%^{1213}$ Rosenqvist et al.," however, found in (healthy) dogs no significant hemodynamic change when the AV interval was varied between 60 and $150 \mathrm{~ms}$. Therefore, the AV interval may be less critical in hearts of young and healthy subjects.

Many studies indicate that abnormal, asynchronous activation of the ventricles plays an important role in the reduction of ventricular function during ventricular pacing. ${ }^{1+10}$ Some of the earlier studies suggest little or no influence of the site of pacing on ventricular performance, ${ }^{1724}$ while other studies report the contrary. Several, more recent, studies show that myocardial function is better preserved when pacing from LV sites than from RV sites. ${ }^{424.32}$

\section{Redistribution of workload during ventricular pacing}

Asynchronous electrical activation alters global LV dynamics, ${ }^{24.31}$ regional wall thickening ${ }^{34}$ and regional wall motion. ${ }^{373536}$ Prinzen et al. ${ }^{3736}$ and Delhaas et al. ${ }^{3040}$ studied the short-term effects of asynchronous electrical activation on regional myocardial function in more detail. The results obtained in these studies are summarized in Figure 2.2. The fiber strain tracings show two important aspects. The onset of local fiber shortening (or: "mechanical activation") is closely linked to the sequence of local electrical 


\section{electrogram}

A
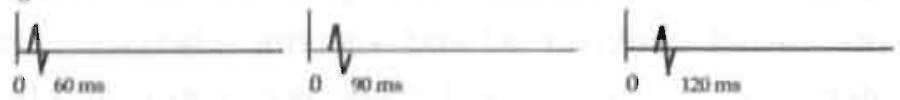

B

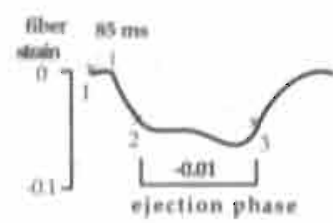

fiber

strom

C
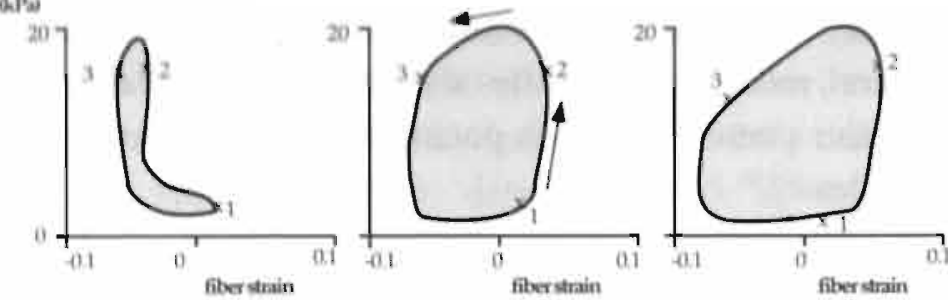

J) total mechanical
work $(\mathrm{mW} / \mathrm{g}$
150

5.00

8.50

blood flow

$(\mathrm{ml} / \mathrm{min} / \mathrm{g})$

0.55

0.70

0.90

oxygen

consumption

(mmol/min/g) 275

3.50

450

early activated normal
Fig 2.2 Seluematic representation of the relationslip betueen sequence of electrical activation, local fiber strain, and mechanical work, based on results from previous studies."

(A) Local electrograms with the pacing artifact (vertical line), local activation complex and the time of electrical activation $(0=$ pacing artifact $)$.

(B) Tracings of local fiber strain. Figures in ms indicate the onset of local fiber shortening ("mechanical activation"), the figures next to the bar indicating the ejection phase depict net fiber strain during the ejection phase (fiber strain $=-0.1$ represents fiber shortening of $10 \%$ ).

(C) Local fiber stress-strain dingrams. Figunes 1,2 and 3 in panels B and C indicate the moments of end-diastole, begin ejection and end ejection of the $L V$, respectively. The area of these diagrams is equal to external mechanical work (hatched area). Total mechamical work was calculated as the sum of external and potential mechanical work."

(D) Average values of total mechanical work, blood flow, and oxygen consumption in the three regions (adapted fron ${ }^{22}$ ).

activation. ${ }^{34}$ Accordingly, changing pacing sites also changes the sequence of mechanical activation. In relatively early activated regions (close to the site of pacing) fibers shorten considerably during the isovolumic contraction phase..$^{37.38}$ During the ejection phase fiber strain in the early activated regions is small (less than -0.05 , as compared to -0.10 during sinus rhythm), probably because these fibers operate at a relatively small sarcomere length when ventricular pressure and wall stress are high. Fiber strain during the ejection phase increases with increasing distance from the pacing site to more than -0.15 in late activated regions, 
due to the prestretch during the isovolumic contraction phase (B, Figure 2.2). Notwithstanding the large differences in local fiber strain during asynchronous electrical activation, systolic fiber stress is not significantly different between early and late activated regions ( $C$, Figure 2.2). Thus, total mechanical work is $50 \%$ lower in early activated regions and $50 \%$ higher in late activated regions, as compared to atrial pacing. The differences found in mechanical load are in agreement with the finding that regional blood flow $w^{3,41}$ as well as oxygen consumption is approximately $30 \%$ lower in early than in late activated regions (D, Figure 2.2). These data show that ventricular pacing induces considerable local differences in mechanical load within the ventricular wall.

\section{Chronic effects of pacing: adaptation?}

Although there is a wealth of information on the short-term effects of pacing, the structural and functional adaptations of the heart to long-term pacing are only sporadically studied. Considering that ventricular pacing considerably affects regional and global myocardial function and that annually 400.000 pacemakers are implanted worldwide, the lack of studies on the effects of chronic ventricular pacing is surprising.
In a study to evaluate battery longevity of ventricular asynchronous pacemakers, 21 healthy dogs were paced chronically and 3 of these dogs died of chronic congestive heart failure, but the time intervals after which these dogs died were regrettably not reported. ${ }^{42}$ In another study 5 months of single-chamber RV pacing in two AV blocked dogs led to diminished cardiac function. ${ }^{4}$

Several clinical studies suggest that the asynchronous activation induced by ventricular pacing leads to detrimental changes in the heart. In this respect large retrospective $\mathrm{e}^{\text {tis }}$ and prospective $\mathrm{e}^{\text {t..123 }}$ studies on long term pacing (5 years) in patients with sick sinus disease are of interest. In these patients both atrial pacing and ventrictilar pacing can be employed. In these studies atrial pacing (AAI) was associated with a significantly better cardiac function, a better NYHA classification, less atrial fibrillation, fewer throboembolic complications, a smaller atrial diameter and the use of less diuretics as compared to ventricular pacing (VVI).$^{4-46,123}$ Furthermore mortality in the ventricular pacing group $(23-30 \%)$ is significantly higher than in the atrial pacing group $(8-13 \%),{ }^{44.45 / 7}$ Part of the differences between AAI and VVI pacing could be explained by the absence of atrio-ventricular coupling in VVI pacing. Santini et al. ${ }^{47}$ compared AAI, DDD and VVI 
pacing. These investigators found that, compared to the AAI group, cardiac mortality was significantly elevated in the VVI, but not in the DDD group. For patients over 70 years of age, however, total mortality was also significantly higher in DDD than in AAI paced patients, while cardiac mortality tended to be higher. Considering the limited size of the groups (ranging from 79 to 135 patients) and the limited period of the study (mean follow-up of 5 years) differences are expected to become more significant in larger scale studies. ${ }^{7}$ Further evidence for adverse effects of asynchronous activation during ventricular pacing was found by Tse et al. ${ }^{+5}$ In a group of 43 patients these investigators found that long term (3.5 year) DDD pacing from the RV apex was associated with myocardial perfusion defects, mainly in the inferior and apical LV region, and apical wall motion abnormalities. The development of these abnormalities was associated with reduction of global LV pump function.

More detailed evidence for (pathophysiological) changes in paced hearts came from studies in young beagles. ${ }^{4050}$ In the immature hearts of these animals chronic epicardial pacing resulted in hemodynamic and histologic changes. These consisted of elevation of right atrial pressure and pulmonary artery pressure, alterations in sinus node function, myofibrillar cellular disarray, dystrophic calcifications, prominent subendocardial Purkinje cells and an increase in variable-sized disorganized mitochondria." Myofiber disarray was also found after pacing in adult canine hearts. In a subsequent study Karpawich et al. ${ }^{50}$ studied the effects of high septal pacing. Under these circumstances there was less asynchronous activation of the ventricles and there were smaller hemodynamic and histological changes than during RV apex pacing. In addition Lee et al. ${ }^{12}$ found that 3 months of RV apex pacing (DDD and VVI) in AV-blocked adult dogs was associated with an increase in tissue catecholamine activity. These observations support the idea that abnormal electrical activation of the ventricles plays an important role in the left ventricular dysfunction seen in cardiac pacing.

In conclusion, pacemakers are nowadays widely used and are of great help to restore heart function in several cardiac diseases. The asynchronous electrical activation induced by ventricular pacing, however, reduces global pump function and leads to a redistribution of myocardial work and blood flow, the long term consequences of which are not well known. The reduction in global pump function may lead to activation of neurohumoral pathways. The redistribution of myocardial work 
may trigger locally acting stimuli leading to structural changes, i.e. regional hypertrophy in the late activated and atrophy in the early activated regions. This was studied in the present thesis. Several types of hypertrophy are often associated with altered myocyte composition, myocardial fibrosis and eventually cardiac failure. Therefore, in the present thesis the changes in cardiac structure and function during chronic ventricular pacing were studied as well. In the next section some aspects of cardiac hypertrophy will be discussed.

\section{CARDIAC HYPERTROPHY}

\section{Introduction}

The heart adapts its structure to changes in hemodynamic demand. The normal human heart increases in weight from approximately $20 \mathrm{~g}$ at birth to about $325 \mathrm{~g}$ in adulthood. ${ }^{51}$ This increase in heart weight is due to the increase in body size and thereby the increased circulatory demand with increasing age. Other increases in cardiac demand may lead to larger heart size as well. Heart weight may rise to about $500 \mathrm{~g}$ in exercise training and even to higher weights in hypertension and valvular stenosis or regurgitation. Up to some days after birth myocytes can increase in number (hyperplasia), but thereafter cardiac growth is mainly due to hypertrophy (increase in cell volume) of myocytes. In extreme hypertrophy, cardiac enlargement may be caused by a combination of myocyte hypertrophy and myocyte hyperplasia." Cardiac hypertrophy may also partly result from proliferation of non-myocytes, i.e. connective tissue cells (fibroblasts, involved in the production of type 1 and type III collagen), vascular cells (smooth muscle cells and endothelial cells), macrophages and mast cells and increased deposition of the extracellular matrix. The increased deposition of collagen, associated with some forms of hypertrophy, may lead to interstitial fibrosis (56-58 for review).

\section{Major forms of hypertrophy}

Based on geometry three different types of hypertrophy can be distinguished. I: Eccentric hypertiophy is generally caused by volume overload. In this form of hypertrophy the increase in wall mass is accompanied by an increase in chamber volume, the ratio of cavity to wall volume is normal. II: Concentric hypertrophy is mainly caused by pressure overload. In this form of hypertrophy the increase in wall mass is due to increased wall thickness, accompanied by normal chamber volume. III: Nonuniform 
Normal

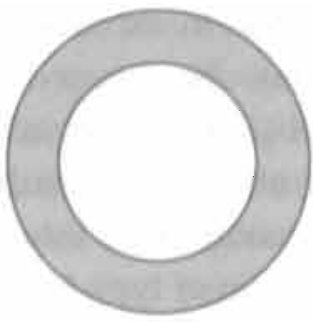

Volume overload hypertrophy

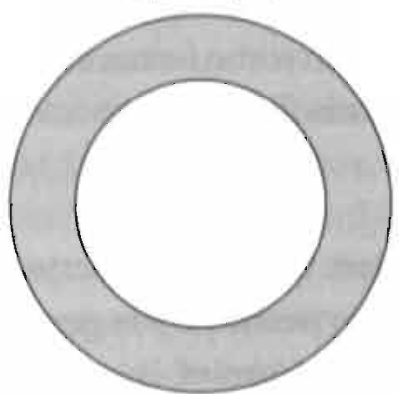

Pressure overload

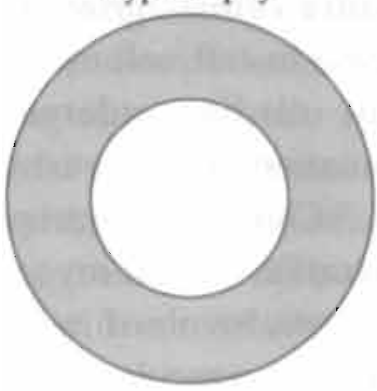

Nonuniform hypertrophy

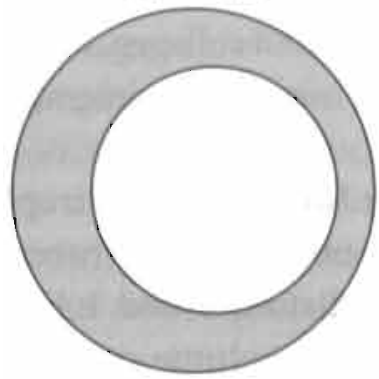
hypertrophy

Figure 2.3 Different forms of hypertrophy.

Top left: drawing of $L V$ cavity and wall geometry of cross section of normal left ventricle. Both volume (bottom left) and pressure overload (top right) lead to hypertrophy, but volume overload results in eccentric hypertrophy and pressure overload in concentric hypertrophy. Non-uniform hypertrophy (bottom, right) occurs, for example, in hypertrophic candiomyopathy. hypertrophy. This type is mostly found in patients with congenital derangements. (Figure 2.3). Based on the cause of hypertrophy various forms of hypertrophy can be distinguished, four of which will be discussed.

\section{1) Volume overload hypertrophy}

Volume overload is prominent in patients with aortic-and mitral-valve insufficiency or atrial-septal defect. Depending on the severity and duration, the volume overload may lead to substantial hypertrophy (increase in heart weight by $60-100 \%$ and even more in aortic regurgitation). In animal studies volume overload hypertrophy has been induced by mitral valve insufficiency ${ }^{30,00}$ aortocaval shunt $t^{50,6)}$ or atrioventricular-block. ${ }^{02-64}$ In volume overload eccentric hypertrophy is found, causing an increase in weight of the LV by $20 \%$ or more and the RV by $40-70 \%$. In compensated volume overload hypertrophy the structure of the myocardium is largely preserved. Hypertrophy in volume overload is mainly caused by an elongation of cardiomyocytes. Collagen fraction and capillary density are not significantly changed. .6.61.05 $^{5.5}$

\section{2) Pressure overload hypertrophy}

In patients and animal models chronic pressure 
overload may be induced by systemic hypertension, aortic stenosis or pulmonary stenosis, and lead to a considerable amount of concentric hypertrophy (heart weight in man > 500 gram). ${ }^{*}$ Pressure overload hypertrophy is generally associated with increased myocyte diameter ${ }^{550}$ and interstitial fibrosis. ${ }^{\text {.M }}$ Because increased myofiber thickness is not associated with an increase in the number of capillaries, capillary density is significantly reduced. Resting myocardial perfusion is generally normal. Increased loading conditions such as tachycardia or strenuous exercise, however, may lead to subendocardial underperfusion. ${ }^{71}$ Hypertrophic hearts due to pressureoverload are also more sensitive to ischemia..$^{72}$

\section{3) Hypertrophic cardiomyopathy (HCM)}

$\mathrm{HCM}$ is characterized by $\mathrm{LV}$ and/or RV hypertrophy, which is usually asymmetric and can affect different regions of the ventricles. Selective hypertrophy of the septum in the subaortic region is most common, leading to the obstructive form of cardiomyopathy (HOCM) and an increased blood pressure gradient between the LV and the aorta. Structural changes include myocyte hypertrophy, myocyte and myofibrillar disarray, abnormal intramural coronary arteries and myocardial fibrosis.
Myocardial perfusion abnormalities, associated with ischemia, have been described. ${ }^{7,4}$ Arrhythmias and premature death may occur. The overall prevalence of HCM is approximately $0.2 \%$, as has been estimated by echocardiography in a large cohort of apparently healthy young adults. HCM is genetically transmitted as an autosomal dominant trait. In a review Bonne et al. ${ }^{70}$ described the molecular basis of the disease. All genetic mutations found so far concern genes coding for sarcomeric proteins: myofilaments, thin-filaments and myosin binding proteins.

\section{4) Exercise induced hypertrophy}

The form of hypertrophy depends on the kind of exercise. In endurance-trained athletes generally eccentric hypertrophy ${ }^{77}$ is present whereas in resistance trained athletes the hypertrophy is concentric. Collagen content, myocardial capillary density, blood flow and coronary resistance are usually normal at least in endurance training induced hypertrophy."

\section{Cardiac (dys)function in LV hypertrophy}

Initially, the development of ventricular hypertrophy is a physiological adaptation to maintain norma! ventricular function. Then the increase in cardiac weight compensates 
fully for the increase in cardiac load. At some point, however, the compensatory mechanisms may not be able to keep up with the increase in load, which may lead to heart failure. Until now, no reliable markers to predict the transition from compensated to decompensated heart function during hypertrophy are available.

In cardiac hypertrophy myocardial substrate preference is shifted from fatty acids toward glucose utilization, while the activity of some metabolic enzymes is altered. Total creatine kinase activity is frequently reduced, leading to a relative high fraction of the isoenzyme creatine kinase-MB, ${ }^{\text {s }}$ while the activity of lactate dehydrogenase may be decreased. ${ }^{.5}$

One of the hypotheses on the origin of heart failure is that in severe LV hypertrophy (LVH) a biological limit of hypertrophy is reached, beyond which hypertrophic compensation to elevated cardiac load is impossible. Carabello et al. showed that ventricular dysfunction was more common in dogs with mitral regurgitation than in dogs with aortic banding, even when they were matched to the same stroke work." The poorer ventricular function was associated with a less pronounced hypertrophy in the mitral regurgitation dogs than in the aorta banded dogs. ${ }^{\circ}$ Also in patients with mitral regurgitation, patients with heart failure had a smaller LV mass to volume ratios than patients without heart failure. ${ }^{\mathrm{r} m}$ It is however not completely understood why hypertrophy was less in the volume overloaded hearts.

Loss of systolic and diastolic cardiac function may also be caused by a disproportionate increase in non-myocyte mass. In various forms of LV pressure overloads, LVH is characterized by reactive fibrosis. ${ }^{57}$

Cardiomyocytes isolated from failing hearts contract poorly, indicating that function is also changed at the myocyte level. Indeed, during hypertrophy, alterations in intracellular myocyte composition have been observed and are suggested to play a role in cardiac failure. These alterations include, amongst others, loss of myofibrils ${ }^{610}$ and an increase in microtubules. ${ }^{8690}$ In small rodents, a shift in myosin ATPase isoforms has been found, leading to a less functional protein..$^{1,92}$ Also modifications in calcium handling proteins have been observed in failing hearts, like a reduction in myocardial ryanodine receptors, sarcoplasmic reticulum Ca2+-ATPase (SERCa) activity ${ }^{94,95}$ and phospholamban activity.

In summary, cardiac hypertrophy constitutes a delicate balance between functional adaptations and potentially deleterious alterations. 


\section{Mechanisms of myocardial hypertrophy}

Myocardial hypertrophy is supposed to be controlled by both neurohumoral factors ${ }^{5,5 e}$ and mechanical load..$^{\text {p.10t }}$ The exact contribution of each of these factors is incompletely understood.

\section{Neurolumoral factors}

Evidence that non-hemodynamic stimuli may induce hypertrophy came from the clinical observation of a poor match between the degree of hypertension and the degree of hypertrophy. ${ }^{* /}$ Such neurohumoral factors as catecholamines, angiotensin, aldosterone, endothelin.102 and thyroxin (103 for review) have been shown to induce hypertrophic response. Part of the hypertrophic response of these substances, however, may be induced by their hemodynamic, mechanical, effect and thus does not represent a direct hypertrophic action. An indication that at least part of the hypertrophy induced by norepinephrine (alpha1-adrenoreceptors) is independent of its hemodynamic activity is that even in quiescent (non beating cells) stimulation causes hypertrophy in neonatal rat. cells.

The renin-angiotensin system (RAS, 105, 106 for review) has been most widely investigated in this respect.
About 2 decades ago different lines of evidence showed that circulating renin-angiotensin either directly or in concert with elevated blood pressure, initiates cardiac hypertrophy. Angiotensin converting enzyme (ACE) inhibitors, preventing the formation of biological active angiotensin II (AngII), proved to be very effective in reversing or preventing the cardiac hypertrophy associated with a state of systemic hypertension. The correlation between the state of activation of the plasma RAS and the hypotensive effect of the ACE inhibitors, however, was unexpectedly low. It is now weil established that in addition to the classical circulating components of the RAS, in the heart, and in many other organs, the RAS is expressed locally (tissue resident RAS). ${ }^{100}$ Events, like myocyte stretch, lead to a local upregulation of the myocardial RAS, including an increase in Angll receptors and Angll formation (105 for review). The locally formed AngII and the circulating AngII are both able to induce a hypertrophic response after binding to myocardial AngII receptors at the myocytes. This hypertrophic response may consist of protein accumulation, ${ }^{110}$ proliferation of fibroblasts and other cell types and increased collagen deposition. Therefore, Ang:II is not only involved in neurohumoral regulation of cardiac growth but also, 
through paracrine and autocrine mechanisms in loadregulated growth of cardiomyocytes.

\section{Mechanical factors}

The notion that hypertrophy occurs in states of increased myocardial workload suggests that mechanical load itself is the trigger for hypertrophy. Because under normal circumstances all cells in the ventricle bear the same load, each cell might react individually to increased load. This concept of hypertrophy regulation at the local level is quite relevant to this thesis, because during asynchronous electrical activation workload in the heart is different locally. Several lines of evidence indicate that hypertrophy can indeed be regulated at the local level. The possibility to control hypertrophy at the local level was evaluated in a mathematical model of LV adaptation to hemodynamic changes." In this model it was assumed that each myocyte and its environment can respond to early systolic length and systolic length changes. Using these assumptions a stable, physiological transmural distribution of fiber direction was obtained as well as appropriate adaptation of wall mass and cavity volume to hemodynamic loading." It was also found that fiber stress is not a compelling condition for the control of cardiac growth.
Also in vitro a stretch induced increase of load leads to a hypertrophic response in myocytes. Stretching of cultured cardiomyocytes increases total mRNA content, which is followed by protein synthesis and cell growth. Stretching of the myocardium also leads to release of growth promoting substances, such as Ang II by myocytes ${ }^{10,112}$ and endothelin by endothelial cells. ${ }^{113}$

Evidence for load regulated cardiac growth in vivo was given by Cooper et al." In a first experimental approach, pulmonary hypertension was evoked by chronic hypoxia. This experimental set-up resulted in selective hypertrophy of the hemodynamically overloaded RV, whereas the LV was not affected. The same results were obtained during beta-blockade or after sympathectomy, indicating that increased hemodynamic load, rather than systemic stimuli, induces hypertrophy. Further evidence that load is an important determinant of the myocardial structure was obtained by in vivo experiments. In these experiments, regional unjoading was achieved by transsection of the chordae tendinae of an intact single RV papillary muscle in cat hearts. Unloading resulted in rapid and marked cellular dedifferentiation. It was found that regional unloading reduces strength and velocity of contraction and increases 
passive stiffness. These changes were fully reversible after reloading of the papillary muscle by re-attachment of the chordae tendinae. ${ }^{\omega}$

\section{Mechanoperception and mechanotransduction}

The concept of load induced hypertrophy requires a sensing mechanism. It is incompletely understood how cells sense mechanical stimuli and how these stimuli are transformed into a biochemical signal inducing the growth response. Deformation of the cell is likely to be important in this respect. Although disputed by some investigators, ${ }^{1 / 4}$ potential transducers of cellular deformation are the cytoskeleton ${ }^{115}$ and stretch-activated channels in the sarcolemma. ${ }^{115}$ Integrins are transmembrane receptors that couple components of the extracellular matrix with the actin cytoskeleton and are also candidates for a role in mechanoperception. ${ }^{100}$ Stretch might also activate membrane bound proteins like adenylate cyclase and phospholipases, enzymes which are related to important second messenger systems involved in the hypertrophic response. ${ }^{100}$

The resulting deformation signal is supposed to be transferred to the nucleus (mechanotransduction), where it can initiate alterations in gene expression. Mechanical load (stretch) as well as growth promoting hormones leads to complex intracellular responses. These include increased intracellular calcium concentration, activation of protein kinases, membrane bound phospholipase C, tyrosine kinases, mitogen-activated kinases $(100,116,117$ for review) and calcineurin. ${ }^{\text {tin }}$ All pathways ultimately lead to induction of immediate-early genes and activation of protein synthesis. Often a fetal gene program is upregulated, such as alpha smooth muscle actin.

Currently it is clear that one trigger (stretch, AngII, for example) gives rise to stimulation of multiple pathways. Generally, several pathways are used by more than one trigger (100, 120 for review). It is incompletely understood, however, why different forms of mechanical overload (pressure or volume overload) lead to different forms of hypertrophy.

\section{CONCLUSION AND RATIONALE}

In summary, neurohumoral factors play an important role in global cardiac hypertrophy and cardiac growth regulation, while the role of local mechanical factors is less clear but likely. The type of hypertrophy largely depends on the kind of perturbations the heart is 
exposed to. Most of this knowledge has been derived from hearts subjected to globally elevated workload, such as hypertension, valvular stenosis or regurgitation. The changes in (regional) structure and geometry due to heterogeneous workload as a consequence of asynchronous electrical activation, however, have been hardly investigated.

In this thesis the major hypothesis to be tested is that in the heart in situ asynchronous electrical activation leads to asymmetric hypertrophy due to an inhomogeneous distribution of workload. This will be investigated in normal (non-hypertrophic) (chapter 5) and hypertrophic hearts (chapter 7). Because hypertrophy, as induced by globally acting stimuli, is often associated with changes in myocardial blood flow and in the activity of metabolic enzymes as well as remodeling of the myocardium including capillarization, myocyte dimensions and collagen content, these aspects were studied as well during chronic ventricular pacing (chapter 6). To study regional myocardial blood flow the fluorescent microsphere technique was further developed (chapter 3) and its applicability in chronic experiments was explored (chapter 4).

Ventricular pacing reduces ventricular function within minutes. Therefore, the effect of chronic pacing on global pump function was investigated as well (chapters 5,7). Finally, it was studied whether in pacing hemodynamic functin can be optimized by proper choice of the site of pacin; or a combination of pacing sites (chapter 8).

\section{REFERENCES}

- Durrer, D, R. T Dam y G. E Freud, M I lanse, E. L Meyler, and R. C Arzbaecher. Total excitatio of the isolated human heart. Circulation. 1970. XI. $1899-912$.

2 Lister, J. W. D H Klot, S. L. Jomain, I H Stuckey, and B. F Hoffman. Effect of pacemaker site on cardiac output and ventricular activation in dogs with complete heart block. Am. I. Cardiol. 1964. 14:494-503.

3. Vassallo, J. A., D. M. Cassidy, F. E. Marchlinski, A. E. Buxton, H. L. Waxman, J. U. Doherty, and M. E. Josephson. Endocandial activation of lefi bundle branch block. Circulation. 1984. 69:914.923.

4. Rosenquist, M. L. Bergfeldt, Y. Haga, J. Rydén, L. Rydên, and A. Cwall. The effect of ventricular activation sequence on cardiac performance during pacing. Pace. $1996,19: 1279-1286$

5. Vassallo, J. A., D. M. Cassidy, J. M. Miller, A. E. Buxton, F. E. Marchlinski, and M. E. Josephson. Left ventricular endocardial activation during right ventricular pacing; effect of underlying heart disease. I. Am. Col!. Cardiol. 1986, 7:1228-1233.

6. Askenazi, I. L. H. Alexander, D. I. Koenigsberg, N. Belic, and M. Lesch Alteration of left ventricular performance by left bundle branch block simulated with atrioventricular sequential pacing. Am. I. Cardicl. 1984. 53:99-104.

7. Resenbush, S W. N. Ruggie, D. A. Tumer, P. L Von Behren, P. Denes, E. W. Fordham, M. W. Groch, and I. V. Messer. Sequence and timing of ventricular wall motion in patients with bundie branch block. Circulation. 1982. 66:113-119.

8. Hirzel, H. O., M. Senn, K. Nuesch, C. Buettner, A. Pfeiffer, O. M. Hess, and H. P. Kravenbuehl. Thallium-201 scintigraphy in complete left bundie branch block. Am. I. Candiol. 1984, 53:76+769.

9. Ono, S, R. Nohara, H. Kambara, K. Okuda, and C. Kawai, Regional myocardial perfusion and glucose metabolism in experimental left bundle branch block. Circulation 1902 85:1125-1131.

10. Samet, P. P. Castillo, and W. H. Bernstein. Hemodynamic consequences of sequential atrioventricular pacing. Am. J Cardiol, 1968, 21:207-212.

11. Faerestrand, 5, and O.-1. Ohm. A time-related study of the hemodynamic benefit of atrioventricular synchronous pacing evaluated by doppler echocardiography. PACE. 1985. 8:838-848. 
12. Masuyama, T., K. Kodama, S. Nakatani, and A. Kitabatake. Effects of atrioventricular interval on left ventricular diastolic filling assessed with pulsed Doppler exhocardiography. Cardiovasc. Res. 1989. 23:1034-1042.

13. Mehta, D., S. Gilmour, D. E. Ward, and A. I. Camm. Optimal atrioventricular delay at rest and during exercise in patients with dual chamber pacemakers: a noninvasive asessment by continuous wave Doppler. Br. Heart J. 1989, 61:161-166.

14. Tanabe, A. T. Mohri, M. Ohga, O. Yoshiga, Y. Hidaka, H. Ikeda, K. Hivamuta, Y. Koga, and H. Toshima. The effects of pacing-induced left bundle branch block on left ventricular systolic and diastolic performances. Ipn. Heart 1. 1990. 31:309-317.

15. Zile, M. R., A. S. Blaustein, G, Shimizu, and W. H. Gaasch. Right ventricular pacing reduces the rate of left ventricular ralaxation and filling. I. Am. Coll. Candiol. 1987. $10-702-709$

16. Bedotto, J. B., P. A. Grayburn, W. H. Black, T. E. Raya, W. McBride, H. H. Hsia, and E. I. Eichhorn. Alterations in left ventricular relaxation during atrioventricular pacing in humans. I. Am. Coll. Cardiol. 1990. 15 (No. 3)-658-664.

17. Miyazawa, K., K. Shirato, T. Haneda, T. Honna, T. Arai, and T. Nakajima. Effects of varying pacemaker sites on left ventricular performance. Tohoku J exp med. 1976. $120: 301-308$

18. Starzl, T. E. R. A. Gaerther, and R. C. Webb. The effects of repetitive electric cardiac stimulation in dogs with normal hearts, complete heart block and experimental cardiac arrest. Circulation. 1955. 11:952-962.

19. Levy, I. M. E. Mesel, and A. M. Rudolph. Unequal right and left ventricular ejection with ectopic beats. Am. J. Physiol. 1962. 203:1141-1144.

20. Fetcher, E. W., E. O. Theilen, M. S. Lawrence, and J. W. Evans. Effect of pacemaker location on cardiac function in complete atrio-ventricular block. Am. J. Physiol. 1963. 205:1232-1234.

21. William-Olsen, G., and M. N. Andersen. The effect of pacemaker electrode site on cardiac output. I. Thoracic Cardiovasc. Surg. 1963. 45:618-621

22. Tsagaris, T. J., R. B. Sutton, and H. Kuida. Hemodynamic effects of varying pacemaker sites. Am. J. Physiol. 1970. 218 (1):246-250.

23. Gibson, D. G., D. A. Chanberlain, D. J. Coltart, and J. Mercer. Effect of changes in ventricular activation on cardiac hemodynamics in man: Comparison of right ventricular, left ventricular, and simultaneous pacing of both ventricles. Brit. Heart J. 1971. $33 \cdot 397-400$.

24. Grover, M. and S. A. Glantz. Endocardial pacing site affects left ventricular enddiastolic volume and performance in the intact anesthetized dog. Circ. Res. 1983. 53:72-85.

25. Daggett, W. M., J. A. Bianco, W. J. Powell, and W. G. Austen. Relative contribution of the atrial systole-ventricular systole interval and of patterns of ventricular activation to ventricular function during electrical pacing of the dog heart. Circ. Res. 1970, 27:69-79.

26. Finney, J. O. Hemodynamic alterations in left ventricular function consequent to ventricular pacing. Am I P'ysiol. 1965. 208:275-282
27. Gilmore, I. P. S. I. Samoff, I. H. Mitchell, and R. I. Linden. Synchronicity of ventricular contraction: observations comparing haemodynamic effects of atrial and ventricular pacing. Brit. Heart I. 1963.25:290-307.

28. Wiggers, C. J. The muscular reactions of the mammalian ventricles to artificial surface stimuli. Am I Physiol. 1925, 73:345-378

29. Park, R. C. W. C. Little, and R. A. O Rourke. Effect of alteration of left ventricular activation sequence on the left ventricular end-systolic pressure-volume relation in closed-chest dogs. Cire Res. 1985. 57;706-717.

30. Little, W. C., R. P Park, and G. L Freman. Effects of regional ischemia and ventricular pacing on $\mathrm{LV}$ dP/dtmax-end-diastolic volume relation. Am. I. Physiol. 1987. 252: $\mathrm{H} 933-\mathrm{H} 940$.

31. Rosenqvist, M., K. Isaaz, E. H. Botvinick, M. W. Dae, I. Cockrell, I. A. Abbott, N. B. Schiller, and J. C. Griffin. Relative importance of activation sequence compared to atrioventricular synchrony in left ventricular function. Am. I. Cardiol. 1991. 67:148-156.

32. Burkhoff, D., R. Y. Oikawa, and K. Sagawa. Influence of pacing site on canine left ventricular contraction. Am. ). Physiol. 1986. 251:H428-H435.

33. Miyazawa, K. T Honna, T. Haneda, K. Shirato, T. Nakajima, and T. Arai, Dynamic geometry of the left ventricle during ventricular pacing: correlation with cardiac pumping action. Tohoku ]. Exp. Med. 1978. 124:261-266.

34. Wilson, I. R. P. Douglas, W. F. Hickey, V. Lanoce, N. Ferraro, A Muhammad, and N. Reichek. Experimental congestive heart failure produced by rapid ventricular pacing in the dog: cardiac effects. Circulation. 1987, 75:857-867.

35. Badke, F. R., P. Boinay, and J. W. Covell. Effect of ventricular pacing on regional left ventricular performance in the dog. Am. I. Physiol. 1980. 238:H858-H867.

36. Little, W. C., R. C. Reeves, J. Anciniegas, R. E. Katholi, and E. W. Rogers. Mechanism of abnomal interventricular septal motion during delayed left ventricular activation. Circ. Res. 1982. 65:1486-1490.

37. Prinzern, E. W. C. H. Augustijn, T Arts, M. A Allessie and R. S Reneman, Redistribution of myocardial fiber strain and blood flow by asynchronous activation. Am. J. Physiol. 1990: 259:H300-H308.

38. Prinzen, F. W. C. H. Augustijn. M. A. Allessie, T. Arts, T. Delhaas, and R. S. Reneman. The time sequence of electrical and mechanical activation during spontaneous beating and ectopic stimulation. Eur. Heart J. 1992. 13:535-543.

39. Delhaas, T., T. Arts, E W. Prinzen, and R. S. Reneman. Relation between regional electrical activation time and subepicardial fiber strain in the canine left ventricle. Eur: J. Physiol. (Pfluegers Arch.). 1993. 423:78-87.

40. Delhaas, T., T. Arts, F. W. Prinzen, and R. S. Reneman. Regional fibre stress-fibre strain area as an estimate of regional blood flow and oxygen demand in the carine heart. I. Physiol. (London). 1994, 477,3:481-496.

41. Beppu. S., H. Matsuda, T. Shishido, and K. Miyatake. Functional myocardial perfusion abnormality induced by left ventricular asynchronous contraction: experimental study using myocardial contrast echocardiography. J. Am. Coll. Cardiol. 1997, 29:1632-1638. 
42. Zhou, J,- L, and G. $-\mathrm{Y}$. Yu. Hemodynamis findings during sinus thythm, atrial and AV sequential pacing compared to ventricular pacing in a dog model. PACE. 1986. 10:118-124.

43. Krayenbuehl, H. P. I. Babotai, H. Mehmel, W. Meier, and M. Turina. Effects of chronic paired pacing upon left ventricular dynamics in the dog. Arch. Kreisiaufforschg, 1971.6423-32

44. Rosenqvist, M. . Brandt, and H. Schullex. Atrial versus ventricular pacing in sinus node disease: A treatment comparison study, Am Heart J. 1986. 111:292-297.

45. Rosenqvist, M., I. Brandt, and H. Schueller. Long-term pacing in sinus node disease effects of stimulation mode on candiovascular morbidity and mortality. Am. Heart I. 1988 . 116.16-22.

46. Nielsen, J. C., H. R. Andenen, P. E B. Thomsen, L. Thuesen, P. T. Mortensen, T. Vesterlund, and A. K. Pedersen. Heart fallure and echocardiographic changes during long-term follow-up of patients with sick sinus syndrome randornized to singlechamber atrial or ventricular pacing. Circulation. 1998, 97-987-995.

47. Santini, M. G. Alexidou, G. Ansalone, C. Cacciatore, R. Cini, and G. Turitto. Retation of prognosis in sick sinus syndrome to age, conduction defects and modes of permanent cardiac pacing. Am. J. Cardiol. 1990. 65:729-735.

48. Tse, H-F, and C-P. Lau. Long-term effect of right ventricular pacing on myocandial perfusion and function. I. Am. Coll Cardiol. 1997. 29:744-749.

49. Karpawich, F. I., C. D. Justice, D. L. Cavitt, and C.H. Chang. Developmental sequelas of fixed-rate ventricular pacing in the immature canine heart: An electrophysiologic, hemodynamic and histopathologic evaluation. Am. Heart J. $1990,119: 1077-1083$

50. Karpawich. P. P. C. D. Justice, C.H. Chang, C. Y. Gause, and L R. Kuhns, Septal ventricular pacing in the immature canine heart: A new perspective. Am. Heart I. 1991. 121:827-833

51. Adomian, G. E. Mvofibrillar disarray produced in normal hearts by chromic electrical pacing. Am Heart 1. 1986. 112:79-83.

52 Lee, M. A. M. W. Dae, I. J. Langberg, J. C. Griffin, M. C. Chin, W. E. Finkbeiner, J. W. OConnell, E. Botvinick, M. M. Scheinman, and M. Rosenqvist. Effects of long. term right ventricular apical pacing on left ventricular perfusion, inneryation. function and histology. J. Am. Coll. Cardiol. 1994. 24:225-232.

53 Vliegen, H. W. Myocardial changes in ventricular hypertrophy. A morphometric, flow cytometric and biochemical study Leiden. The Netherlands. 1987.

54. Linzbach, A. I. Heart failure from the point of view of quantitative anatomy. Am. 1. Card. 1060. (march) $370-382$

55. Anversa, P. I. Kaistura, W. Cheng, K. Reiss, E Cigola, and G. Olivetti Insulin-like growth factor-l and myocyte growth: the danger of a dogma Part II. Induced myocardial growth: pathologic hypertrophy, Cardiovasc. Res, 1996, 32:484-495.

56. Weber, K. T., and C. G. Brilla. Pathological hypertrophy and cardiac interstitium. Fibrosis and renin-angiotensin-aldosterone system. Circulation. 1991. 83:1849-1865.

57. Brilla. C. G. and B Maisch. Regulation of the structural remodelling of the myourdium: from hypertrophy to heart failure. Eur. Heart J. 1994. 15 (suppl. D) $45-52$.
58. Weber, K. I., Y.S. Sun, and E Guarda. Structural remodeling in hypertensive heart disease and the role of hormones. Hypertension 199423 (part 2) $869-877$

59. Carabello, B. A., M. R. Zile, R. Tanaka, and G. Cooper IV Left ventricular hypertrophy due to volume overload vensus presoure overlosd. Am. I. Fhysiol. 1992. 263:H1137-H1144.

60. Urabe, Y, D. I Manm, R. I Kent, K. Nakano, R. I. Tomanek, B. A. Carabello, and C. Cooper IV. Cellular and ventricular contractile dysfunction in experimental canine mitral regurgitation. Circ. Res. 1992. 70:131-147

61. Legault. F. I. L Rouleau, C luneau, C Rose, and K. Rakusan Functional and morphological characteristics of compensated and decompensated cardiac hypertrophy in dogs with chronic infrarenal aorto-caval fistulas. Circ. Res. 1990 $66,846-859$

62. Wusten, B, W. Flameng, B. Winkier, and W. Schaper. Role of cardiac contractility in hypertrophy from volume overloading. Cardiovasc Res. 1977. 11:132-140.

63. Newman, W. H. Volume overload heart failure: length-tension curves, and the response to beta-agonists, Ca2+, and glucagon. Am. J. Physiol. 1976. 235.H690H700.

64. Vos, M. A. S. H. M. de Groot, S. C. Verduyn, J van der Zande, H. D. M. Leunissen, I. P. M. Cleutjens, M. van Bilzen, M. J. A. P. Daemen, J. J. Schreuder. M. A. Allessie, and H. I. J. Wellens. Enhanced susceptibility for acquired torsade de pointes arrbytmias in the dog with chronic, complete AV block is related to cardiac hypertrophy and electrical remodeling. Circulation. 1998. 98.1125-1135.

65. LeWinter, M. M. R. L Engler, and I, S, Karliner. Enhanced left ventricular shortening during chronic volume overload in conscious dogs. Am I Physiol. 1980. 238: $\mathrm{H126- \textrm {H } 1 3 3}$

66. Vliegen, H. W., A. Laarse van der, J. A. N. Huysman, E. C. Wijnvoord, M. Mentar: C. J. Comelisse, and F. Eulderink. Morphometric quantification of myocyte dimensions validated in normal growing rat hearts and applied to hypertrophic human hearts. Cardiovasc. Res. 1987. 21:352-357.

67. Anversa, P., R. Ricci, and. G. Olivetti. Quantitative structural analysis of the myocardium during physiological growth and induced cardiac hypertrophy. ). Am. Coll Cardiol. 1986. 7:1140-1149.

68. Brilla, C. G., R. Pick, L. P. Tan, J. S. Janicki, and K. T. Weber. Remodeling of the rat right and left ventricles in experimental hypertension. Circ. Res. 1990. 67:13551364 .

69. Rakusan, K., P. W. Hrdina, Z. Turek, E. G. Lakatta, H. A. Spurgeon, and G. D. Wolford. Cell size and capillary supply of the hypertensive rat heart: quantitative study. Basic. Res, Cardiol. 1984. 79:389-395.

70. Tomanek, R. L. K A Schalk, M. L Marcus, and D G Harrison. Coronary angiogenesis during long-term hypertension and left ventricular hypertrophy in dogs, Cine Res. 1989, 65352-359.

71. Hittinger, L. I. Mirsky, Y-T Shen. T. A. Patrick, S. P. Bishop, and S. F Vatner. Hemodynamic mechanisms responsible for reduced subendocardial coronary reserve in dogs with severe left ventricular hypertrophy. Cinculation. 1995, 92-978-986. 
72. Snoeckx, L. H. E. H. G. J, van der Vusse, W. A. Coumans, and R. S. Reneman. The effects of global ischemia and reperfusion on compensated hypertrophied hearts of aorta-banded rats. J. Mol. Cell. Cardiol. 1990. 22:1439-1451.

73. Wigle, E. D. H. Rakowskd, B. P. Kimball, and W. G. Williams. Hypertrophic cardiomyopathy. Clinical spectrum and treatment. Circulation 1995. 92-1680-1692

74. Kappenberger, L. C Linde, C. Daubert, W. McKerna, E Meisel, N. Sadoul, I. Choinowska. L. Guize, D. Gras, X. Jeanrenaud, L. Ryden, and a. t. P. s. group. Pacing in hypertrophic obstructive cardiomyopathy. Eur Heart J. 1997. 18:12491256.

75. Maron, B. J. f. M. Gardin, J. M. Flack, S. S. Gidding, I. T. Kurosaki, and D. E. Bild Prevalence of hypertrophic candiomyopathy in a general population of young adults: echocardiographic analysis of 4111 subjects in the CARDIA study. Circulation. 1995, 92:785-789.

76. Bonne, G., L. Carrier, P. Richard, B. Hainque, and K. Schwartz. Familial hypertrophic cardiomyopathy. From mutations to functional defects. Circ. Res. 1998. 83:580-593

77. Snoeckx, L. H. E. H. H. F. M. Abeling, J. A. C. Lambregts, J. I. F. Schmitsz, F. T. J. Verstappen, and R. S. Reneman. Cardiac dimensions in athletes in relation to variations in their training program. Eur. J. Appl. Physiol. 1983. 52:20-28.

78. George, K. P. L. A. Wolfe, and G. W. Burggral. The "athletic heart syndrome", a critical review: Sports medecine. 1991. 11 (5) 300-331

79. Mall, G., G. Zimmer, S. Baden, and I. Mattfeldt. Capillary neoformation in the ra! heart-stereological studies on papillary muscles in hypertrophy and physiological growth. Basic Res, Cardiol. 1990. 85:521-540.

80. Kagaya, Y. Y. Kanno, D. Takeyama, N. Ishide, Y. Muruyama, T. Takahashi, T. Ido. and T. Takishima. Effects of long-term pressure overload on regional myocardial glucose and free fatty acid uptake in rats. a quantative autoradiographic study. Circulation. 1990, 81:1353-1361

81. Taegtmeyer, H., and M. L. Overturf. Effects of moderate hypertension on cardiac function and mefabolism in the rabbit, Hypertension. 1988. 11:416-426.

82. Bishop, S. P. and R. A. Altschuld. Increased glycolytic metabolism in cardiac hypertrophy and congestive failure, Am J Physiol. 1970. 218:153-159.

83. Smith, S. H., M. F. Kramer, I. Reis, S. F. Bishop, and I. S. Ingwall. Regional changes in creatine kinase and myocyte size in hypertensive and nonhypertensive cardiac hypertrophy. Circ. Res, 1990. 67:1334-1344.

84. Ingwall, J. S., M. F. Kramer, M. A. Fifer, B. H. Lorell, R. Shemin, W. Grossman, and P. D. Allen. The creatine kinase system in normal and diseased human myocardium. N. Fngl. 1. Med. 1985; 313:1050-1054.

85. Laarse van der, A., L. Hollaar, H. W. Vliegen, J. M. Egas, N. J. Dijkshoorn, C. J. Comelisse, A. J I. C Bogers, and I. M. Quagebeur Myocardial (iso)enzyme activities, DNA concentration and nuclear polyploidy in hearts of patients operated upon for congenital heart disease, and in normal and hypertrophic adult human hearts at autopsy. Eur J Clin Invest. 1989. 19:192-200.
86. Tagawa, H. M. Koide, H. Sato, M. R. Zile, B. A. Carabello, and G. Cooper IV. Cytoskeletal role in the transition from compensated to decompensated hypertrophy during adult canine left ventricular pressure overicading. Cire Res. 1998. $82-751-761$.

87. Corin, W. L. E S Monrad, T Murakami, H. Nonogi, O. M. Hess and H. P Krayenbuehl. The relationship of afterload to ejection performance in chronic mitral regurgitation. Circulation. 1987. 76:59-67.

88. Schuller, G. K. L Peterson, A. Johnson, G. Francis, G. Dennish, / Utely, P O. Daily W. Ashburn, and J. Ross. Temporal response of left ventricular performance to mitral valve surgery, Circulation. 1979. 59:1218-1231

89. Wong, C. Y. H. and H, M. Spotnitz. Systolic and diastolic properties of the humnan left ventricle during valve replacement for chronic mitral ngurgitation. Am. J. Candiol. 1981. 47:40-50

90. Tsutsui, H. H. Tagawa, R. L Kent, P. L McCollam, K. Ishihara, M. Nagatsu, and G. Cooper IV. Role of microtubules in contractile dysfunction of hypertmphied candiocytes Circulation. 1994. 90:533-555.

91. Dom II, G. W., J. Robbins, N. Ball, and R. A. Walsh. Myosin heavy chain regulation and myocyte contractile depression after LV hypertrophy in aortic banded mice. Am. I. Physiol. 1994. 267:H400-H405

92. Swynghedauw, B. K. Schwartz, and C. S. Apstein. Decreased contractility after myocardial hypertrophy: cardiac failure or successful adaptation? Am, J. Cardiol. 1984. $54,437-440$

93 Vatner, D. E, N. Sato, K. Kiuchi, R. P. Shannon, and S E Vatmer Decruase in myocardial ryanodine receptors and aftered excitation-contraction coupling early in the development of heart failure. Circulation. 1994. 90:1423-1430.

94. Bastie, D. d. I., D. Levitsky, L Rappaport, I-1. Mercadier, F. Marotte, C. Wisnewsky, V. Brovkovich, K. Schwartz and A. Lompré. Function to the sarcoplasmic reticulum and expression of its Caz+-ATPase Gene in pressure overload-induced. cardiac hypertrophy in the rat. Circ, Res, 1990, 66:554-564.

95. Oi, M. T R. Shannon, D. E. Fuler, D M Bers, and A. M Samarel Downregulation of sarcoplasmic reticulum $\mathrm{Ca}_{2}+$-ATPase during progression of left ventricular hypertrophy. Am. I. Physial. 1997. 272:H2416-H2424.

96. Kiss, E., N. A. Ball, E. G. Kranias, and R. A. Walsh. Differential changes in cardiac phospholamban and sarcoplasmic reticular $\mathrm{C} 2 \mathrm{2}$-ATPase protein levels. Effects on Ca2 + transport and mechanics in compensated pressure-overload hypertrophy and congestive heart failure. Circ Res, 1995, 77:759-764.

97. Dzau, V. I. Tissue renin-angiotensin system in myocardial hypertrophy and failure. Arch. Int. Med. 1993. 153:937-942

98. Long, C. S., K. Kariya, L. Karns, and P. C. Simpson. Trophic factors for cardiac myocytes. J. Hypertension. 1990. 8 (suppl. 7):S219-\$223.

99. Cooper IV, G. Cardiocyte adaptation to chronically altered load. Ann. Rev. Physiol. 1987. $49: 501-518$ 
100.Sadoshima, J4 and \$. Izumo. The cellular and molecular response of cardiac myocytes to mechanical stress. Annu. Rev. Physiol. 1997. 59:551-571.

10I.Kent, R. L, and P. I. McDermott. Passive load and angiotensin II evoke differentia nesponses of gene expression and protein synthesis in cardiac myocytes. Cinc. Res. 1996. 78:829-838.

102. Harada, M. H. Itoh, O, Nakagawa, Y. Ogawa, Y. Miyamoto, K. Kuwahara, F Ogawa, T. Igaki, I. Yamashita, I. Masuda, T. Yoshimasa, I. Tanaka, Y. Saito, and K Nakao. Significance of ventricular myocytes and nonmyocytes interaction during candiocyle hypertrophy. Evidence for endothelin-1 as a paracrine hypertrophic factor from candiac nonmyocyles. Circulation. 1997.96:3737-3744.

103. Morgan, H. E, and K. M. Baker. Cardiac hypertrophy. Mechanical, neural and endocrine dependence. Circulation. 1991, 83(1):13-25.

104.Clark, W. A. S. I Rudnick. I I LaPres, L. C. Andersen, and M. C. LaPointe Regulation of hypertrophy and atrophy in cultured adult heart cells. Circ. Res. 1993, 73:1163-1167

105. Venkatesh, K., B. S. Raman, Y, A. Lee, and K. Lindpaintner. The candiac renin aneiotensinaldosterone system and hypertensive cardiac hypertrophy. Am. Cardiol. 1995, 76:18D-23D.

106. Ruzicka, M., B. Yuan, E. Harmsen, and E. H. H. Leenen. The renin-angiotensin system and volume overload-induced cardiac hypertrophy in rats: Etfects of angiotensin converting enzyme inhibition versus angiotensin II receptor blockade. Circulation. 1993, 87:921-930

107 Schunkert, H. B. Jackson, S. S. Tang, F. J. Schoen, J. F. M. Smits, C. S. Apstein, and B. H. Lorell. Distribution and functional significance of cardiac angiotensin converting enzyme in hypertrophied rat hearts. Circulation. 1993. 87:1328-1339.

108. Lee, Y. A., C. S. Liang, M. A. Lee, and K. Lindpaintner. Local stress, not systematic factors, regulate gene expression of the cardiac renin- angiotensin system in vivo: A comprehensive study of all its components in the dog. Proc. Natl. Acad. Sci. 1996. 93:11035-1040.

109.Iwai, N. H. Shimoike, and M. Kinoshita. Cardiac renin-angiotension system in. the hypertrophied heart. Circulation. 1995, 92:2690-2696.

110. Schumkert. H I I Sadoshima, T Comelins, Y Kagaya, E. O. Weinberg, S. Inumo, G. Riegger, and B. H. Lorell. Angiotensin H-induced growth responses in isolated adult rat hearts. Evidence for load-independent induction of candiac protein synthesis by angiotensin II. Circ. Res, 1995, 76:489-497.

111.Arts, T., F. W. Prinzen, L H. E. H. Snoeckx, I. M. Rijcken, and R. S. Reneman Adaptation of cardiac structure by mechanical feedback in the environment of the cell: A model study. Biophysical f. 1994. 66:953-961.

112. Sadoshima, I Y Xu, H S Slayter, and S Izumo Autocrine release of angiotensin 11 mediates stretch-induced hypertrophy of cardiac myocytes in vitro. Cell. 1993. $75: 977.984$

113.Carosi. J. A., S, G. Eskin, and L. V. Mclntire. Cyclical strain effects on production of vasagctive materials in cultured endothelial cells. I. Cell Physiol. 1992 151:29. 36.
114.Sadoshima, J. 1. T. Takahash L. Jahu, and S. Lzumo. Roles of mechano-sensitive ion channels, cytoskeleton, ad contractile activity in stretch-induced immediategene expression and hypertrohy of cardiac myocytrs. Proc. Natl. Acad. Sci. 1992. 89.9095 .9979 .

115. Watson, P. A. Function follors form: generation of intracellular signals by cell deformation. FASEB I. $1991+2013-2019$

116.Sugden, P. H., and M. A. Begoyevitch, Intracellular signalling through protein kinases in the heart. Cardiovsc. Rev. 1995. 30-478-492.

117.van Bilsen, M. Signal transdction revisited: recent developments in angiotensin II signaling in the cardiovasular system. Cardiovasc. Res. 1997. 36:310-322

118.Sussman, M. A., H. W. Lim N. Gude, T. Taigen, E. N. Olson, J. Robbins, M. C. Colbert, A. Gualberto, D. F. lieczorek, and J. D. Molkentin. Prevention of cardiac hypertrophy in mice by calcieurin inhibition. Science. 1998. 281(5383):1690-1693.

119.Molkentin, f. D. I. R. Lu. C. I Antos, B. Markham, I. Richardson, I. Robbins, 5. R. Grant, and E. N. Olson. A alcineurin-dependent transcriptional pathway for cardiac hypertrophy, Cell 198, 93:215,228.

120. Reneman, R. S. T Arts, M an Bilsen, L H. F H Snoecks, and G I san der Vusse. 1995. Mechanoperception and mechanotransduction in cardiac adaptation: mechanical and molecular aspects. In Molecular and subcellular cardiology: Effects of structure and function. S. Sideman, and R. Beyar, editors. Plenum Press, New York. 185-193.

121.Prinzen, E. W., M. F. M. yan Oosterhout, T. Arts, and R. S. Reneman. Local functional and structural changes in the myocardium during ventricular pacing. 1. interven Cardiol. 1996, 9:319-326.

122. Bernstein, A. D., A. I. Camm, and R. D. Fletcher. The NASPE/BPEG generic pacemaker code for antibradyarrhytmia and adaptational pacing in antitachyarrhytmia devices. PACE. 1987. 10:794.

23. Andersen HR, Nielsen KC. Thomsen PFB, Thuesen L, Mortensen PT Vesterlund T Pedersen AK. Long-term follow-up of patients from a randomised trial of atrial versus ventricular pacing for sick-sinus syndrome. Lancet. 1997;350:1210-1216. 


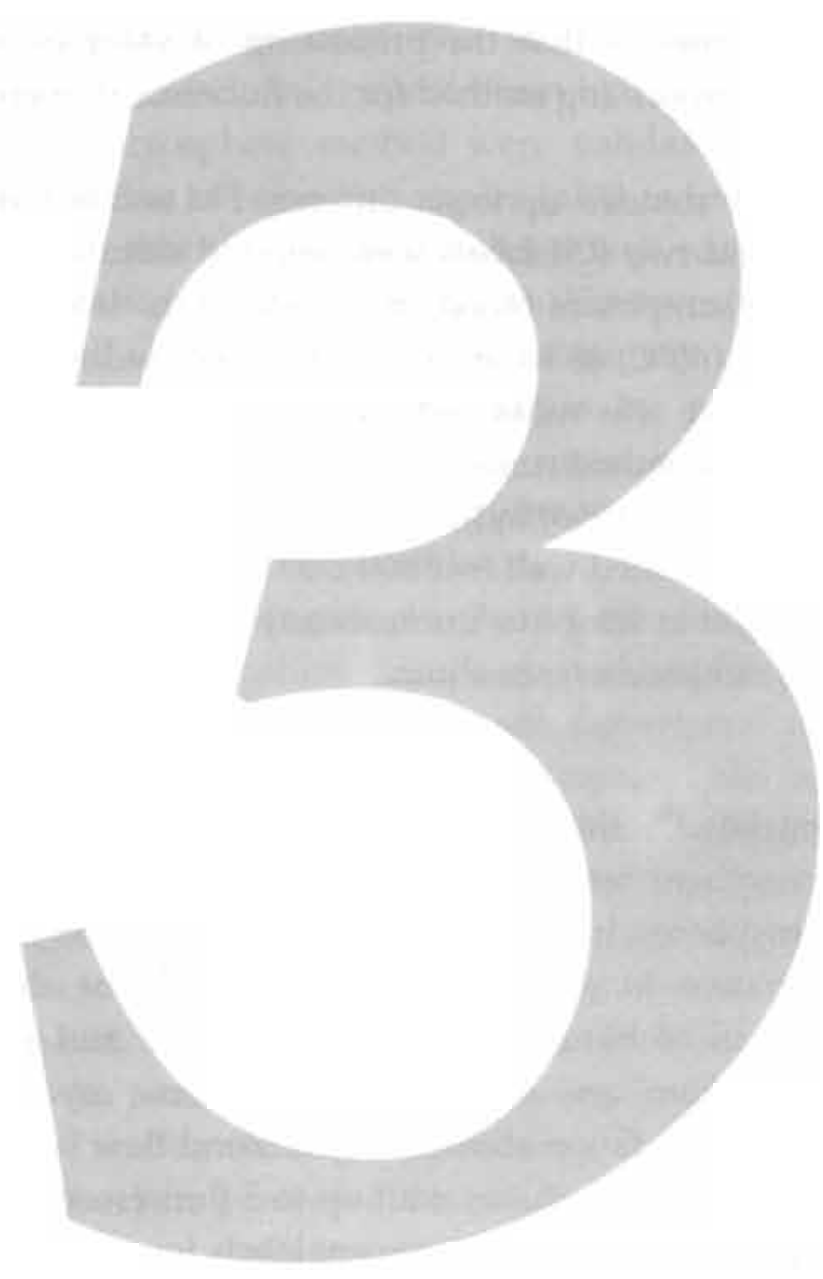

\section{FLUORESCENT MICROSPHERES TO MEASURE ORGAN PERFUSION: VALIDATION OF A SIMPLIFIED SAMPLE PROCESSING TECHNIQUE}

M.F.M. van Oosterhout, H.M.M. Willigers,

R.S. Reneman, F.W. Prinzen

Cardiovascular Research Institute Maastricht (CARIM), The Netherlands

(Am. J. Physiol, 269:H725-H733, 1995) 
Background - A disadvantage of non-radioactive microsphere techniques is that the processing of samples is timeconsuming and complex. We developed and validated a simplified processing method for the fluorescent microsphere (FM) technique.

Methods and Resuits - In seven anesthetized dogs with coronary artery stenosis up to six different FM and five different radioactively labeled microspheres (RM) were injected. Two FM and two RM labels were injected simultaneously to enable inter- and intramethod comparison. After gamma-counting samples of blood, myocardium ( $\mathrm{n}=168$ ) and other organs $(n=59)$ were digested in test tubes with $2 \mathrm{~N}$ ethanolic $\mathrm{KOH} \quad\left(60^{\circ} \mathrm{C}, 48 \mathrm{~h}\right)$, microspheres were sedimentated by centrifugation, dye was extracted in the same tube, and fluorescence was measured. With this processing method, recovery of FM was nearly $100 \%$. Good correlations for inter- and intramethod comparisons were found, $[\mathrm{r}=0.985 \pm 0.01$ (mean \pm s.d.)]. The lower intermethod correlation for blue microspheres $(r=0.958)$ indicate that the use of this label is less desirable, RM and FM endocardial-to-epicardial blood flow ratios correlated well ( $\mathrm{r}=0.974)$.

Conclusions - With this one-vessel centrifugal sedimentation method and at least five fluorescently labeled microspheres, blood flow can be reliably measured in various organs, including ischemic myocardium.

$\mathbf{R}$ adioactive microspheres are used to measure regional organ blood flow. This method has been validated in many studies ( 8 and 14 for reviews) and is currently regarded as a "gold standard" for (regional) blood flow measurements in experimental cardiovascular research. The use of radioactivity is becoming increasingly problematic due to restrictive legislation and higher costs of storage and disposal. These factors, along with the desire to reduce employee radiation exposure, have stimulated the development of nonradioactive microsphere techniques. Recently, fluorescently labeled microspheres have been demonstrated to be a reliable alternative to radioactive microspheres for the determination of blood flow in lung, kidney and normoxic myocardium 7 and normoxic and ischemic myocardium. ${ }^{2}$ In these validation studies, organ blood flow was estimated using microspheres with up to 5 fluorescent labels for lung perfusion and 3 fluorescent labels for myocardial and 
kidney perfusion. In only one study ${ }^{2}$ blood flow measurements in ischemic myocardium by means of the fluorescent microsphere method were validated. In a restricted number of ischemic samples ( $n=11$ in four dogs) it was shown that the results correlated well with those obtained with radioactive microspheres. However, no data were reported on the validity of the assessment of endocardial-to-epicardial blood flow ratios by means of fluorescent microspheres. Blood flow measurement with fluorescent microspheres in other organs, such as the liver, brain and skin, has not been validated.

Before quantifying the number of microspheres in a sample, a microsphere isolation and purification procedure is required. For this purpose, digestion of solid tissues and blood with $\mathrm{KOH}{ }^{1,7}$ or proteases ${ }^{2}$ has been used. Subsequently the homogenate is isolated by negative pressure filtration ${ }^{7}$ or the use of sucrose gradients. ${ }^{2}$ As has been suggested before? the disadvantage of these procedures is that sample processing does not take place in a single vial, which could result in loss of microspheres. Moreover, the procedures are relatively expensive (filters, proteinases) and time consuming. When fatty tissues (e.g., brain and liver) are processed, filters are easily occluded.

This paper describes and validates a simplified processing technique in which tissue digestion, microsphere isolation, and dye extraction are performed in one vessel and in which centrifugal sedimentation rather than microfiltration is used. The number of spheres is quantified by extracting the dyes in the solvent and subsequently measuring the fluorescence. This method is validated in canine nonischemic and ischemic myocardium, as well as in various other organs, using microspheres labeled with up to six different fluorescent dyes.

\section{METHODS}

\section{Animal preparation}

The experiments were performed on 7 mongrel dogs of either sex and unknown age, ranging in weight from 24 to $35 \mathrm{~kg}$. The animals were premedicated with Fentanyl $(20 \mu \mathrm{g} / \mathrm{kg}$ body wt im). Anesthesia was induced with thiopental sodium $330 \mathrm{mg} / \mathrm{kg}$ body wt iv) and, after endotracheal intubation, was maintained with nitrous oxide in oxygen ( 60 :40 by volume) and halothane 0.5 $1 \%$ using a volume controlled respirator (Servo 900, Siemens). The preparation has been described in detail before. ${ }^{12}$ in short, the heart was exposed through a left anterior thoracotomy. An inflatable occluder cuff. was placed around the left anterior descending coronary artery (LAD) to induce a pressure-controled stenosis." Saline-filled catheters were placed in the left atrium for microsphere injection and in the femoral artery for taking reference blood samples. 


\section{Microsphere injection}

In each experiment microspheres with blue, blue-green, yellow-green, orange, red and crimson fluorescent labels (polystyrene, $15.5 \mu \mathrm{m} \pm 2 \%$. Molecular Probes (Eugene, OR)), and microspheres with a maximum of five different isotopes: ${ }^{141} \mathrm{Ce}$, ${ }^{113} \mathrm{Sn},{ }^{103} \mathrm{Ru}^{95} \mathrm{Nb}$ and ${ }^{46} \mathrm{Sc}(15.5 \pm 0.1 \mu \mathrm{m}$, New England Nuclear $)$ were injected into the left atrium. For each determination about $3.10^{6}$ microspheres per fluorescent or radioactive label were injected. In one experiment a double number of blue microspheres $\left(6 \cdot 10^{6}\right)$ was injected. Before injection, the radioactively and fluorescently labeled microspheres, suspended in $10 \%$ dextran with $0.05 \%$ Tween 80 , were mixed, sonicated, and vortexed. To allow calculation of absolute blood flow rates, a reference blood sample was taken from the femoral artery at a rate of $20.7 \mathrm{ml} / \mathrm{min}$ with a Harvard suction pump. Withdrawal of blood started $5 \mathrm{~s}$ before injection of the microspheres and was continued for $\geq$ one minute after microsphere injection.

\section{Experimental protocol}

After the surgical procedure and hemodynamic stabilization, the LAD was narrowed by the occluder (distal LAD pressure $=40 \%$ of mean aortic pressure), producing an area of myocardial hypoperfusion. For intermethod (fluorescent vs. radioactive) and intramethod (fluorescent vs. fluorescent and radioactive vs. radioactive) evaluation, microspheres labeled with two different fluorescent dyes and two different radioactive isotopes were mixed in one syringe and injected simultaneously during stabilized I.AD stenosis. Up to 4 other fluorescent and up to 3 other radioactive labels were injected at other time intervals, either during coronary stenosis or after deflation of the cuff. Only the results of the simultaneously injected microsphere pairs are discussed.

The experiments were terminated by administration of an overdose of pentobarbital sodium. From 5 experiments the hearts. were excised and stored at $-20^{\circ}$ or $5^{\circ} \mathrm{C}$ for further processing. Before dissection, nonmuscular structures like fat and vessels at the epicardium as well as chordae tendinae were removed. From each heart about 12 transmural pieces were obtained from the hypoperfused anterior left ventricular wall (ischemic samples) and from the normally perfused posterior wall (nonischemic samples). Each transmural sample was divided into three layers of equal thickness, i.e, the subendocardial, mesocardial, and subepicardial layers, and each subsample was weighed (a total of $n=168$ ). From three experiments samples from skin, kidney, brain, spleen, skeletal muscle, gut, and lung (referred to as 'other organs'; in total $n=59$ ) were collected and weighed. Note that in this protocol "lung blood flow" refers to the microspheres delivered through the bronchial circulation and the pulmonary artery, the latter after shunting through AV anastomoses.

The radioactivity of the tissue and arterial blood samples was determined in a gamma counter (LKB 1282 Compugamma, Wallac).

\section{Tissue and blood digestion}

All tissue samples and reference blood samples were transferred to regular $10 \mathrm{ml}$ glass, screw cap, test tubes (caps with Teflon inlay because of the aggressive reactants used). 


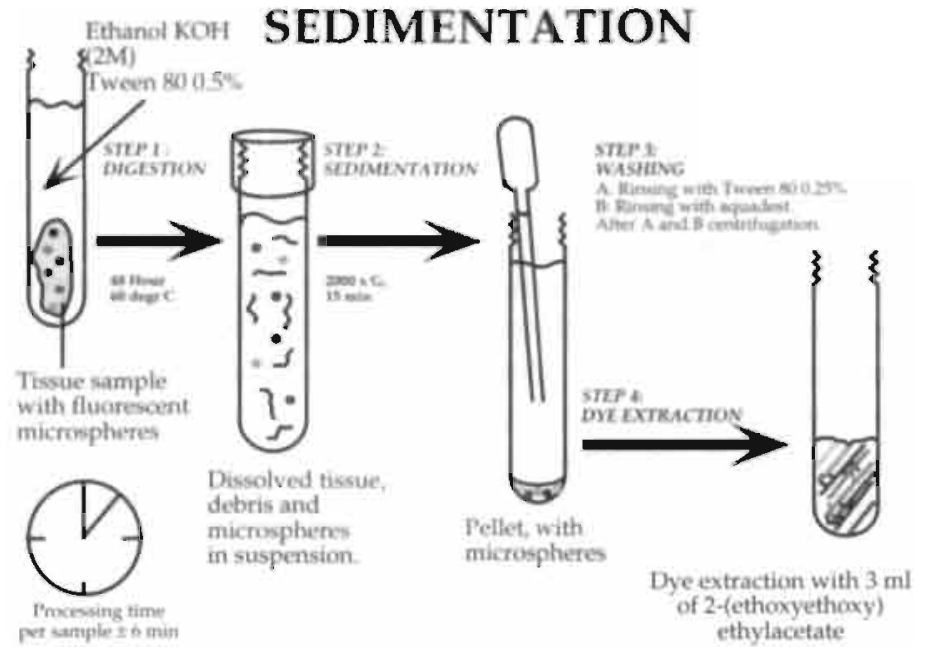

Nonmyocardial samples were allowed to autolyze (without $\mathrm{KOH}$ ) at room temperature in a fumehood for about one week. Myocardium, reference blood, and, after autolysis, other organs were digested in $2 \mathrm{~N}$ ethanolic $\mathrm{KOH}$ (Sigma) with $0.5 \%$ Tween 80 . The ratio of tissue or blood to digestion medium was $\leq 1: 3$. To enhance digestion, the tubes were placed in a $60^{\circ} \mathrm{C}$ water bath for $48 \mathrm{~h}$ and manually shaken after $24 \mathrm{~h}$. Then the tubes with homogenized samples were centrifuged ( $20 \mathrm{~min}, 2000 \mathrm{~g}$ in a swingout bucket centrifuge), and the supernatant carefully removed with a suction system, leaving $<1 \mathrm{ml}$. The difference in specific gravity between digestion medium $\left(0.893 \mathrm{~g} / \mathrm{cm}^{3}\right)$ and fluorescent microspheres $\left(1.055 \mathrm{~g} / \mathrm{cm}^{3}\right)$ allowed the formation of a pellet after centrifugal sedimentation. This pellet, containing microspheres and some debris, was rinsed with $9 \mathrm{ml} 0.25 \%$ Tween 80 in demineralized water $\left(60^{\circ} \mathrm{C}\right)$, vortexed, and centrifuged again at the same force and duration. The pellet was completely resuspended before centrifugation. A last rinsing step was performed with demineralized water without-Tween 80 . After centrifugation, the supernatant was carefully removed while preventing disturbance of the pellet. To avoid clumping, the pellet was not dried, but $<0.5$ $\mathrm{ml}$ was left. Finally, $3 \mathrm{ml}$ of an organic solvent, 2-(2-ethoxyethoxy)ethyl acetate (Aldrich Chemical, Milwaukee, WI), was added to the pellet to extract the fluorescent dye from the microspheres. Tubes were vortexed and allowed to stand for $\geq$ $4 \mathrm{~h}$, vortexed again, and centrifuged (10 mirc, $2000 \mathrm{~g}$ ), leaving a clear solvent in which fluorescence was determined. Fluorescence of myocardial tissue and reference blood samples of one experiment was determined at the same day, whereas fluorescence of the other organs was determined later.

\section{Microsphere recovery}

To confirm that no microspheres were lost during the procedure of digestion and subsequent sedimentation with supernatant removal, two tests were performed. In the first test, the supernatant. of all rinsing steps from the samples of one experiment. was collected and filtered (filter device, Molecular Probes). Fluorescence of any retained microspheres was determined by soaking the filters in solvent $(3 \mathrm{ml})$ and determining the fluorescence. In the second test, $200 \mu \mathrm{l}$ of a suspension containing, orange microspheres was added to empty vials and, as an internal. reference, to vials containing myocardial samples from an experiment in which dyes other than orange had been used. The 
myocardial samples were processed according to the sedimentation method. Orange fluorescence from the microspheres alone (without further processing) and those combined with tissue samples (after sample processing) were compared.

\section{Determination of fluorescence}

Fluorescence was determined with a Perkin-Elmer LS-50B luminescence spectrophotometer with an excitation wavelength range from 200 to $800 \mathrm{~nm}$ and an emission wavelength range from 200 to $900 \mathrm{~nm}$. The machine was equipped with a pulsed xenon light source, variable excitation and emission monochrometers with variable slit widths, a red-sensitive photo multiplier tube (wavelength range $200-900 \mathrm{~nm}$ ) and a cuvette reader or a flow cell (L2:15-1247, Perkin-Elmer). All fluorescence measurements were made with excitation and emission slit widths of $6 \mathrm{~nm}$ and an emission filter blocking all light below $350 \mathrm{~nm}$. The optimal excitation and emission wavelengths for each of the six fluorescent dyes were determined.

To determine the concentration of microspheres in suspension, an aliquot of microspheres of each dye was counted manually with a fluorescence microscope. The counted microspheres were dissolved in $3 \mathrm{ml}$ of solvent, and the fluorescence intensity was determined in a cuvette $(10 \mathrm{~mm}$ path length) or in a flowcell. For each dye the fluorescence per microsphere per $\mathrm{ml}$ solvent was calculated. With these 'standards', a spillover matrix was constructed, and the linearity of the fluorescence signal as a function of microsphere concentration was determined.

In serial measurements the FAC4A software package (developed by Perkin-Elmer and the Fluorescent Microsphere Resource Center, University of Washington, Seattle) allowed automatic scanning of each sample for the 6 specific excitation and emission wavelength pairs.

\section{Blood flow calculation}

Blood flow values for radioactive and fluorescent microspheres were calculated after subtracting radioactive and fluorescent background from raw data, respectively, making corrections for spectral spillover by means of the matrix inversion method." For the fluorescent data, blood flow values with and without correction for spillover were compared. Because no significant difference in blood flow values derived from fluorescent microspheres with and without correction could be detected, the uncorrected data were used.

For myocardial samples, blood flow was calculated with the formula

$$
\mathrm{Q}_{i}=\left(\mathrm{Q}_{\mathrm{ref}} \cdot \operatorname{Int}_{i} / \ln \mathrm{t}_{\mathrm{ref}}\right)
$$

where $Q_{i}$ and $Q_{\text {ref }}$ is the flow in sample $i$ and the reference withdrawal speed, respectively, and Int; and Int $t_{\text {ref }}$ are the radioactivity or fluorescence intensity in sample $i$ and in the reference blood sample, respectively. Myocardial blood flow values are expressed in $\mathrm{ml} \cdot \mathrm{min}^{-1} \cdot$ sample $^{-1}$, or normalized per unit of weight and expressed. in $\mathrm{ml} \cdot \mathrm{min}^{-1} \cdot \mathrm{g}^{-1}$.

The ratio of blood flow $\left(\mathrm{ml} \cdot \mathrm{min}^{-1} \cdot \mathrm{g}^{-1}\right)$ in subendocardial. and subepicardial samples was calculated and defined as the endocardial-to-epicardial ratio.

For the other (non-myocardial) organ samples the relative 
$\left(Q_{\mathrm{rel}_{i}}\right)$ blood flow was calculated using the formula

$$
Q_{\text {rel }_{i}}=\left(\operatorname{Int}_{i} / \operatorname{lnt}_{\text {mean }}\right) \cdot \text { weight }_{i}^{-1}
$$

where Int $t_{i}$ is fluorescence or radioactivity in sample $i$, Int tmean $_{\text {is }}$ mean fluorescence or radioactivity of all organ samples from each experiment, and wheight $t_{i}$ is the weight of sample $i$. For these organs, relative blood flow was used rather than absolute blood flow because the fluorescence of the other organs was not measured on the same day as that of the arterial reference samples. As will be shown, repeatability of fluorescence measurements is less when

Fig 3.1 Inter-method evaluation. Regional myocardial perfusion as determined by simultaneous injection of microspheres labeled with two different fluores-cent and two different radioactive labels each in onte experiment. Blood flow per myocardial sample (in mt-min'sample'). A: Regression plot for inter-method evaluation of the blood flow measured with fluorescent microspheres $\left(Q_{m}\right)$ as a function of the mean value of blood flow determined with niobium ("Nb)- and tin ("'Sn)-labeled microspheres ( $\overline{\mathrm{Q}} \mathrm{s})$. Blood flow was measured in samples from ischemic (subscript i) and non-ischentic (subscript ni) myo-cardium with yellow-green- [YG and YG.j] and with crimson ICR and $C R$ - l labeled microspheres, respectively. The regression equations for ischemic and non-ischemic samples together are: $\bar{Q}=0.05+$ $0.98 \mathrm{Q}$; standard error of estimate $(S E E)=0.022 ; r=0.992$; and $\bar{Q}=0.01$ $+0.99 \mathrm{Q}$ o; $S E E=0.031 ; r=0.984 . B:$ Absolute intermethod error as a function of mean walue of blood flow determined with " $\mathrm{Nb}$-, "Sn-, YG- and CRlabeled microspheres $(\bar{Q})$ Absolute intermethod error $=\bar{Q}_{\mathrm{si}}-\mathrm{Q}_{\mathrm{iai}}=-0.04 \pm$ 0.05 (mean $\pm S D$, in ml-min -samplet) $^{-1}, \bar{Q}-Q_{a}=-0.00 \pm 0.07$ for ischemic samples, and $\bar{Q}_{u}-Q_{\text {row }}=-0.02 \pm 0.08$ and $\bar{Q}_{m}-Q_{0 m}=0.01 \pm 0.12$ for non-ischemic samples.

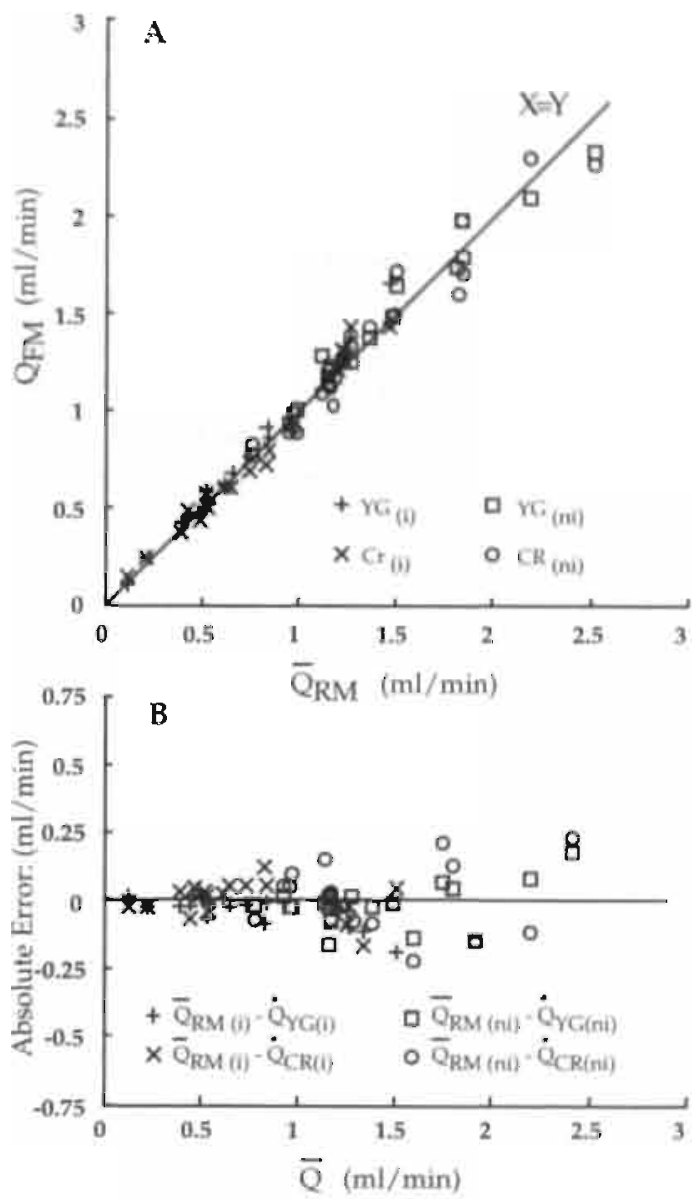


tissue and reference samples are measured on different days rather than on the same day (see Discussion). Absolute blood flow in the other organs, however, could be derived from the radioactive microspheres.

\section{Statistics}

Blood flows determined with each of the two simultaneously injected fluorescently labeled microspheres $\left(Q_{F M_{1} \text { or } 2}\right)$ were compared with the mean of the blood flow values obtained from the two simultaneously injected radioactively labeled microspheres ( $\left.\bar{Q}_{R M}\right)$. $\bar{Q}_{R M}$ was used rather than $\hat{Q}_{R M}$ or 2 from individual isotopes to obtain the most precise blood flow standard. Because the error made with microspheres is related to the number of microspheres per sample, the use of data from two labels will increase accuracy, especially in ischemic samples (see also sources of error in DISCUSSION). For intra- and intermethod comparison, linear regression analysis was performed. Regression coefficients ( $r$ ) and standard error of estimate (SEE) were calculated for the best-fitting lines. The slopes of the lines were compared with unity and the intercepts were compared with the origin. Data were also compared using the analysis of Bland and Altman, where the difference (which is the absolute error) between the two methods ( $\bar{Q}_{R M}-Q_{F M} M_{1 \text { or } 2}$ for intermethod comparison and $\overline{Q R M}_{1}-\overline{Q R M}_{2}$ or QFM - QFM $M_{2}$ for intramethod comparison) was plotted against the average of $\hat{Q R M}_{1}, Q_{R M_{2}}, Q_{F M}$ and $\hat{Q F M}_{2}$ (mean flow: $\bar{Q}$ ). For each individual experiment the mean absolute error \pm standard deviation (SD) was calculated and tested for significant deviation from 0 . The mean absolute error is a measure for systematic overor underestimation of one of the methods; the inter- or intramethod variability is expressed by the SD of the difference. Significance was tested with an unpaired double tailed $t$-test, and $p<0.05$ was considered significant. The intermethod error was also calculated by means of the mean absolute error ( $\mathrm{Err}_{\mathrm{abs}}=\overline{\mathrm{Q}} \mathrm{RM}-\overline{\mathrm{Q}} \mathrm{FM}$ ) and the mean relative error $\left[\mathrm{Err}_{\mathrm{rel}}=200\left(\overline{\mathrm{Q}}_{\mathrm{RM}}-\overline{\mathrm{Q}}_{\mathrm{FM}} / \overline{\mathrm{Q}}_{\mathrm{RM}}+\overline{\mathrm{Q}}_{\mathrm{FM}}\right)\right]$ in both ischemic and nonischemic areas. For most error calculations and regression analysis blood flow per sample was used. To evaluate the effect of flow rate on the accuracy of blood flow measurements, different symbols were used for ischemic and nonischemic samples in plots (Figs. 3.1 and 3.2) and error calculations were performed for ischemic and nonischemic samples separately. To give an impression of the degree of ischemia, blood flow ( $\overline{\mathrm{Q}} \mathrm{RM}$ and $\overline{\mathrm{Q}} \mathrm{FM}$ ) in the subepicardial, mesocardial and subendocardial layers in ischemic and nonischemic myocardium was normalized per gram tissue and expressed in $\mathrm{ml} \cdot \mathrm{min}^{-1} \cdot \mathrm{g}^{-1}$.

The variability of repeated fluorescent signals was quantified by the coefficient of variation $\left(C_{i}\right)$.

\section{RESULTS}

\section{Digestion of tissue}

Myocardium, weighing 0.4-3. g, and reference blood readily digested in $7 \mathrm{ml} .2 \mathrm{~N}$ ethanolic $\mathrm{KOH}$ within $48 \mathrm{~h}$. After one wk of autolyzing without $\mathrm{KOH}$ all other organ tissues softened and weakened, which facilitated subsequent digestion with ethanolic $\mathrm{KOH}$. When large (about $3 \mathrm{~g}$ ) tissue samples of fatty tissue (intestine, liver, or 
skin) were digested, a fatty debris occasionally floated on top of the ethanolic $\mathrm{KOH}$ after $48 \mathrm{~h}$ of digestion. These samples could be completely digested by subdividing the content of one tube over four tubes and repeating the incubation in ethanolic $\mathrm{KOH}$ at $60^{\circ} \mathrm{C}$.

\section{Microsphere recovery}

Filtration of the supernatant from 36 myocardial samples was found to contain $<1 \%$ of the total fluorescence. When, in another test, orange microspheres were added to the tissue samples as an internal reference before digestion and sedimentation, on the average fluorescence was $4 \%$ lower than the fluorescence of the same number of microspheres directly extracted (15.5 \pm 0.9 and $16.1 \pm 0.7$, respectively). This difference, however, was not statistically significant. Both tests showed that there was no significant loss of microspheres with the supernatant, and the recovery of microspheres was about $100 \%$.

\section{Duration and costs of analysis}

When processing a series of 50 samples, the average time for processing one sample with the sedimentation method required $6 \mathrm{~min}$ and costs about $\$ 0.17$ (US).

\section{Fluorescence measurements}

The variability of repeated measurements $(n=10)$ of fluorescent signals from the same sample, as described by $C_{v}$, was $0.3-1.5$ and $0.2-1.1 \%$ for the cuvettes and flowcell, respectively, when measured on the same day. If the same sample was measured every day for 7 consecutive days, the $C_{v}$ was $2.0-9.2 \%$. With the use of optimal

\begin{tabular}{|lrrrrrr|}
\hline \multicolumn{7}{l|}{ Table 3.1: Spillover matrix of fluorescent colors } \\
\hline Color & Blue & $\begin{array}{r}\text { Blue- } \\
\text { Green }\end{array}$ & $\begin{array}{r}\text { Green- Orange } \\
\text { Ged }\end{array}$ & Crimson \\
\hline Blue & 100.0 & 0.0 & 0.0 & 0.0 & 0.0 & 0.0 \\
Blue-Green & 0.8 & 100.0 & 0.1 & 0.0 & 0.0 & 0.0 \\
Yellow-Green & 0.0 & 0.0 & 100.0 & 0.1 & 0.0 & 0.0 \\
Orange & 0.0 & 0.0 & 0.0 & 100.0 & 0.4 & 0.0 \\
Red & 0.0 & 0.0 & 0.0 & 0.5 & 100.0 & 0.5 \\
Crimson & 0.0 & 0.0 & 0.0 & 0.0 & 3.2 & 100.0 \\
Intensity 100sph/ml & 10.26 & 10.49 & 31.57 & 14.12 & 8.52 & 4.39 \\
Excitation (nm) & 358 & 431 & 496 & 530 & 570 & 614 \\
Emission (nm) & 420 & 466 & 506 & 555 & 600 & 639 \\
& & & & & & \\
\hline
\end{tabular}

Table 3.1 For the construction of the spillover table, fluorescence of pure samples was measured in a cuvette or in a flowcell (no difference in spillover, fuores-cence of flowcell $80 \%$ compared to fluorescence measured in cuvette), using excitation and emission wavelength pairs as indicated. Spillover is given in percentages. Fluorescence of 100 microspheres dissolved in $1 \mathrm{ml}$ of solvent neasured in a cuvette is presented. 

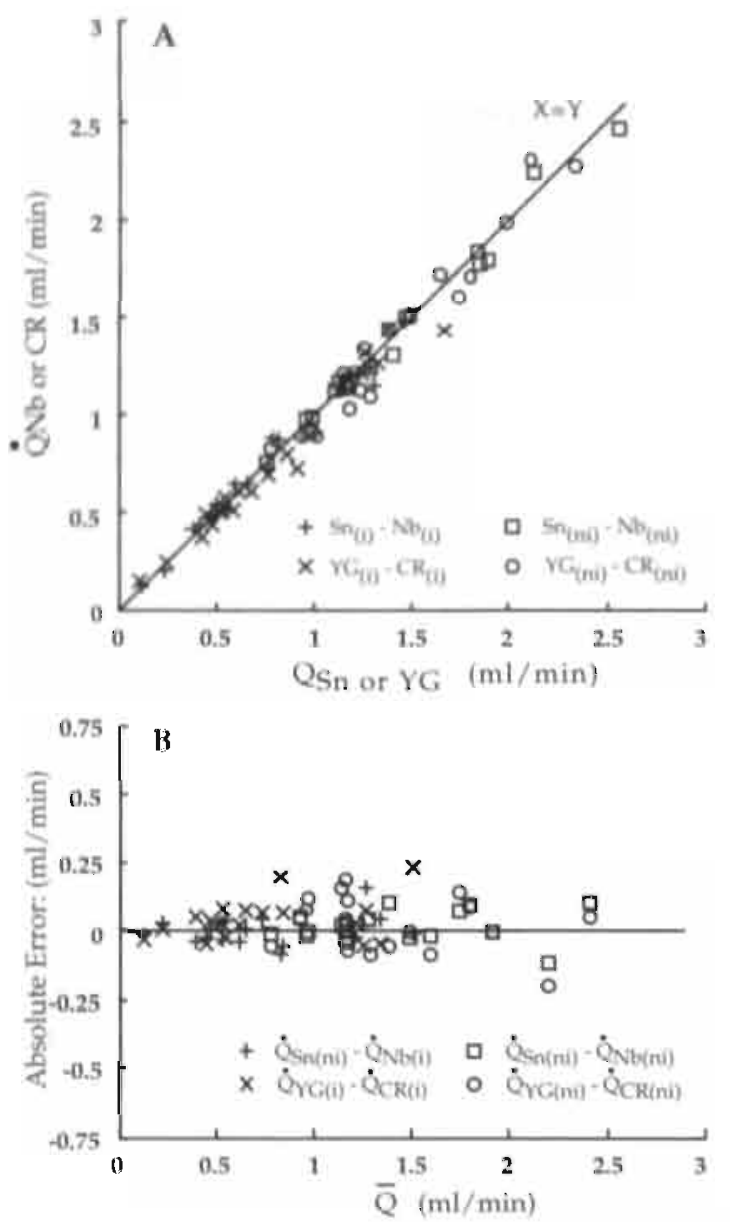

excitation and emission wavelengths for each dye, the spillover of different fluorescent dyes was small and only substantially larger than zero for crimson to red: $3.2 \%$ (Table 3.1). There was a difference in fluorescence per microsphere between colors. The lowest values were found for red and crimson, and the highest values were found for yellow-green and orange (Table 3.1 shows values as measured in a cuvette). Fluorescence measured in the flowcell was $80 \%$ of that measured in a cuvette. Fluorescence intensity ranged from 7 to 400 for all organ and reference blood samples. When fluorescence was $>200$, samples were diluted to be sure that the measure-

Fig3.2 Intra-method comparison. Same experiment and abbreviations as fig 1; blood flow given per myocardial sample ( $\mathrm{ml}^{-} \cdot \mathrm{min}^{2}$-sample'). A: Blood flow meastired with "Nb-labeled microspheres $1 Q$ voo or $Q$ wow l plotted as a function of blood flow measured with "tsu-labeled microspheres $[Q$ mo or $Q$-mil for evaluation of the radioactive intra-method variation. Intra-method evaluation for fluorescent method shown as well; $I Q$ ran or $Q$ mam is plotted as a function of $\dot{Q}$ an or $Q$ and. The regression equations for ischemic and non-ischemic samples together are: $Q_{s}=0.023+0.972 Q s ;$ SEE $=0.053 ; r=0.995$; and $Q a=-0.035+1.00 Q$ rc; SEE $=0.092 ; r=0.986 . B:$ Absolute intra-method error as a function of the mean value of blood flow determined with "Nb-, "Sn-, YG and CR-labeled microspheres $(\bar{Q})$. Absolute intra-method error =

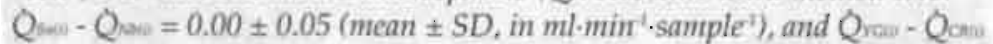

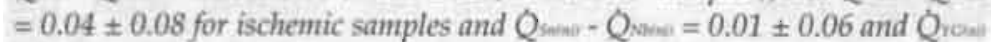
- $Q$ anw $=0.02 \pm 0.10$ for non-ischemic samples. 


\begin{tabular}{|c|c|c|c|}
\hline Sample type & Slope & htercept & Correlation \\
\hline \multicolumn{4}{|l|}{ Myocardial } \\
\hline$\widetilde{Q}_{\text {est vs, }}$ Qru & $0.98 \pm 0.03$ & $004 \pm 0.06$ & $0.985 \pm 0.01$ \\
\hline$Q_{4}$ vs. Qm & $0.99 \pm 0.04$ & $001 \pm 0.07$ & $0.981 \pm 0.02$ \\
\hline Qun vs. Q & $0.99 \pm 0.02$ & $001 \pm 0.01$ & $0.997 \pm 0.00$ \\
\hline \multicolumn{4}{|l|}{ Other organs } \\
\hline Quvs. Q & $0.99 \pm 0.05$ & $-601 \pm 0.01$ & $0.982 \pm 0.01$ \\
\hline
\end{tabular}

Table 3.2 Values are means \pm SD. Regnession analysis of the flow values in myocar-dium ( $n=168$ samples) and variove other organs ( $n=59$ samples) are shown. $Q$ m and $Q_{m}$, blood flow determined with radioactive and fluorescent microspheres, respectively. Pooled data from all experiments are presented.

ments were made in the fluorescence range where the relation between microsphere concentration and fluorescence signal was linear.

\section{Myocardial perfusion studies}

Blood flow values obtained with fluorescent microspheres correlated very well with those obtained with radioactive microspheres, Figure 3.1 shows the data for one typical experiment, and in Table 2 the pooled data for all experiments are presented $(r=0.985 \pm 0.02$, slope and intercept not significantly different from 1 and 0 respectively). In these experiments, blood flow varied from 0.1 to $2.5 \mathrm{ml}=\mathrm{min}^{-1}=\mathrm{g}^{-1}$ myocardial tissue. The intramethod correlation is shown in Fig. 3.2 (the same experiment as Fig. 3.1), and in Table 3.2 the pooled data for all experiments are presented. In one experiment (expt. 5) the data of one of the two simultaneously injected radio-

\begin{tabular}{|c|c|c|c|c|}
\hline Experiment & $\overline{\mathrm{Q}}_{\mathrm{ssr}-\mathrm{Q}_{\mathrm{rm}}}$ & 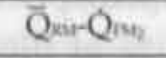 & $Q_{1+1}-Q_{n}$ & $Q_{\text {nat, }}-Q_{\text {new }}$ \\
\hline 1 & $\begin{array}{c}\text { OR } \\
-0.03 \pm 0.09\end{array}$ & $\begin{array}{c}\text { CR } \\
-0.04 \pm 0.07\end{array}$ & $\begin{array}{c}\text { OR-CR } \\
0.01 \pm 0.06\end{array}$ & $\begin{array}{c}\mathrm{Sn}-\mathrm{Nb} \\
0.01 \pm 0.02\end{array}$ \\
\hline 2 & $\begin{array}{c}Y G \\
0.01 \pm 0.16\end{array}$ & $\begin{array}{c}\text { CR } \\
0.04 \pm 0.18\end{array}$ & $\begin{array}{l}\text { YG-CR } \\
0.03 \pm 0.16\end{array}$ & $\begin{array}{c}\mathrm{Ce}-\mathrm{Nb} \\
0.00 \pm 0.04\end{array}$ \\
\hline 3 & $\begin{array}{c}\text { BL * } \\
-0.16 \pm 0.11\end{array}$ & $\begin{array}{c}\text { RD } \\
0.00 \pm 0.05\end{array}$ & $\begin{array}{c}\text { BL-RD * } \\
-0.16 \pm 0.12\end{array}$ & $\begin{array}{c}\mathrm{Sn}-\mathrm{Ru} \\
0.01 \pm 0.04\end{array}$ \\
\hline 4 & $\begin{array}{c}Y G^{*} \\
-0.03 \pm 0.07\end{array}$ & $\begin{array}{c}\text { CR: } \\
0.00 \pm 0.10\end{array}$ & $\begin{array}{l}\text { YG-CR * } \\
0.03 \pm 0.02\end{array}$ & $\begin{array}{c}\mathrm{Sn-Nb} \\
0.00 \pm 0.06\end{array}$ \\
\hline 5 & $\begin{array}{l}\mathrm{Ce}-\mathrm{BL}^{*} \\
0.11 \pm 0.20\end{array}$ & $\begin{array}{l}\text { Ce-BG * } \\
0.05 \pm 0.15\end{array}$ & $\begin{array}{c}\text { BL-BG }^{*} \\
-0.06 \pm 0.13\end{array}$ & \\
\hline
\end{tabular}

Table 3.3 Valies are mean differences $\pm S D\left(\mathrm{ml}^{2} \cdot \mathrm{min}^{-}\right.$-sample' $)$. Inter- and intra-method error of myocardial blood flow determination for each experiment separately. $\bar{Q} \mathrm{su}$, mean flow determined with two radioisotopes, $Q$ $r_{1}=2$, flow determined with one of the two simultancously injected fluorescent micro-spheres (fluorescent dyes used: blue (BL), Btue-green (BG), yellow-green $(Y G)$, orange ( $O R$ ), red $(R D)$, and crimson ( $C R)$; radioisotopes

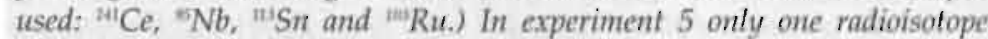
("'Ce) was used. "P $<0.05$ vs, zero. 
active labeled microspheres $\left({ }^{103} \mathrm{Ru}\right)$ were discarded because of unacceptable low radioactivity of this isotope.? For the pooled data the intramethod correlation for the fluorescent method was slightly lower $(r=0.981 \pm 0.01)$ than for the radioactive method $(r=0.997 \pm 0.00)$. In one experiment (expt. 3) the inter- and intramethod correlation for fluorescently labeled microspheres was 0.958 and 0.946 respectively, due to the use of blue microspheres. If the data obtained with the blue microspheres in experiment 3 are discarded, then the pooled intermethod correlation $\left(\overline{\mathrm{Q}}_{\mathrm{RM}}-\mathrm{Q}_{\mathrm{FM}}\right)$ became 0.988 and the intra-method correlation $\left(\dot{Q}_{\mathrm{FM}_{1}}-\dot{\mathrm{Q}}_{\mathrm{FM}}\right) 0.990$.

Figures $3.1 \mathrm{~B}$ and $3.2 \mathrm{~B}$ show plots of the absolute error plotted as a function of blood flow for inter- and intra-method comparison, respectively. Table 3.3 summarizes the values of the mean absolute error for the individual experiments. The pooled data as well as the data for individual experiments show that for most

Table 3.4: Blood flow and intermethod error in nonischemic and ischemic myocardium

\begin{tabular}{|c|c|c|c|c|c|c|c|c|}
\hline \multirow{3}{*}{ 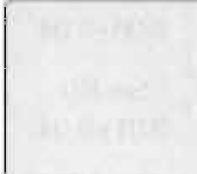 } & \multicolumn{4}{|c|}{ Nonischemic } & \multicolumn{4}{|c|}{ Ischemic } \\
\hline & \multicolumn{3}{|c|}{ 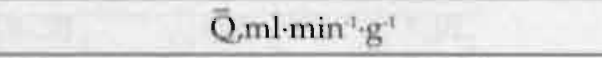 } & & \multicolumn{3}{|c|}{$\overline{\mathrm{Q}}, \mathrm{ml}^{\prime} \cdot \mathrm{min}^{-1} \cdot \mathrm{g}^{\prime}$} & \multirow[b]{2}{*}{ Endo/Epi } \\
\hline & Epi & Meso & Endo & Endo/Epi & Epi & Meso & Endo & \\
\hline RM & $1.12 \pm 0.35$ & $1.30 \pm 0.22$ & $1.48 \pm 0.21$ & $1.38 \pm 0.31$ & $0.90 \pm 0.60$ & $0.56 \pm 0.50$ & $0.38 \pm 0.32$ & $0.44 \pm 0.26$ \\
\hline \multirow[t]{2}{*}{$\mathrm{FM}$} & $1.13 \pm 0.30$ & $1.35 \pm 0.18$ & $1.42 \pm 0.17$ & $1.36 \pm 0.31$ & $0.89 \pm 0.55$ & $0.58 \pm 0.45$ & $0.41 \pm 0.30$ & $0.44 \pm 0.22$ \\
\hline & \multicolumn{2}{|c|}{ Offset } & \multicolumn{2}{|c|}{ Variation } & \multicolumn{2}{|c|}{ Offset } & \multicolumn{2}{|c|}{ Variation } \\
\hline \multirow{2}{*}{\multicolumn{2}{|c|}{$\begin{array}{l}\text { Err. } \mathrm{ml} \cdot \mathrm{min}^{-1} \text { sample' } \\
\mathrm{Err}_{n, i} \%\end{array}$}} & \multirow{2}{*}{$\begin{array}{l}0.02 \pm 0.06 \\
0.32 \pm 2.58\end{array}$} & & \multicolumn{2}{|c|}{$0.00 \pm 0.04$} & \multicolumn{2}{|c|}{$0.07 \pm 0.04$} \\
\hline & & & \multicolumn{2}{|c|}{$8.25 \pm 3.51$} & \multicolumn{2}{|c|}{$-4.46 \pm 10.31$} & \multicolumn{2}{|c|}{$16.16 \pm 15.57(\%)$} \\
\hline
\end{tabular}

Table 3.4 Values are means \pm SD of pooled data from all myocardial samples (except flow values obtained from blue microspheres in experiment 3 ). $\bar{Q}$ mean blood flow assessed with microspheres labeled with two radioactive labels (RM) or with two fluorescent labels (FM), in epicardial (Epi), mesocardial (Meso),

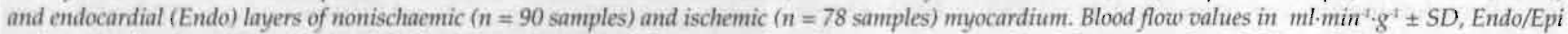
= the ratio of endocandial and epicardial blood flow. Systemic differences and random errors between blood flow assessed with RM and FM are represented by offset and variation, respectively. Err, absolute intermethod error; Err, relative intermethod error. 


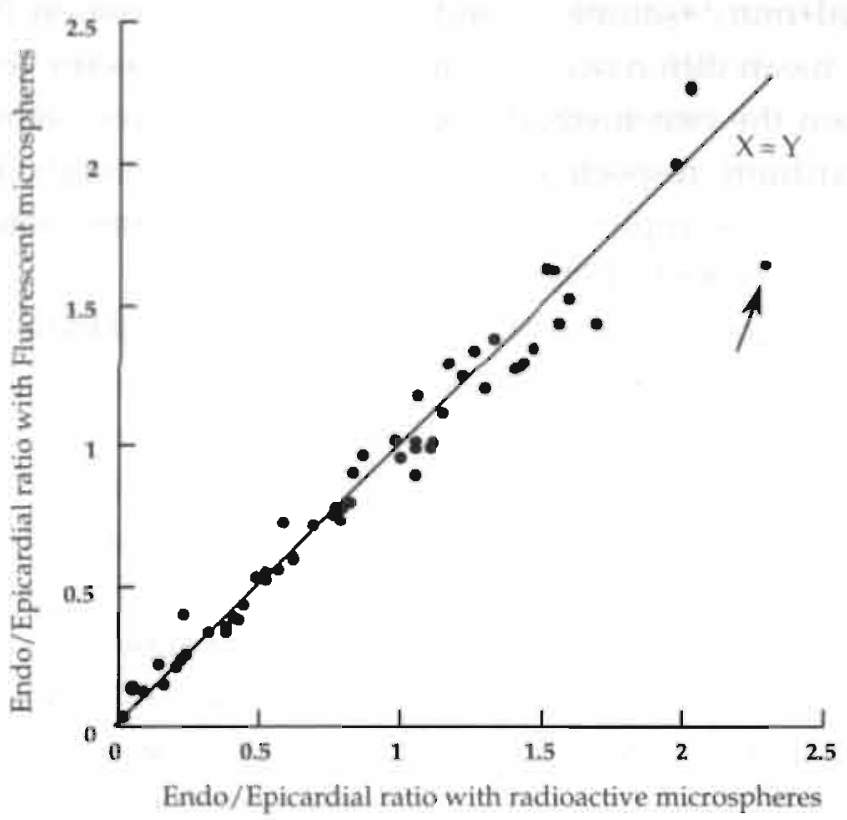

Fig 3.3 Endocandial (Endo) to-epicardial blood flow ratios determined by simultaneous injection of microspheres labeled with two different fluorescent and two different radioactive labels each per experiment. Plot shows pooled data from all experiments; line of unity is plotted. Regression equation for all data points is $y=0.91 x+0.07 ; r=0.974$. Discarding one stray point (arrow), the equation changes to $y=0.96 x+0.03 ; r=0.985$, slope and intercept are not statistically different from identity and origin, respectively. experiments there was no systematic over- or underestimation or large random variation in the fluorescent method compared with the radioactive method. However, blue microspheres led to a slight but significant overestimation of blood flow in experiment 3 and a underestimation of blood flow in experiment 5.

In Fig. 3.1A, the data points from the ischemic myocardial samples scatter around the same line as those from the nonischemic samples. Similarly, Fig. 3.1B shows that the absolute error between the flow values determined with fluorescently and radioactively labeled microspheres at a given flow per sample is similar for ischemic and nonischemic myocardial samples. This indicates that the error between the radioactive and fluorescent methods is independent of flow rate. This aspect is further elaborated in Table 3.4, in which the statistical analysis of the pooled blood flow data for ischemic and nonischemic myocardium is presented.

Mean blood flow in all layers was 0.62 in ischemic myocardium and $1.3 \mathrm{ml} \cdot \mathrm{min}^{-1} \cdot \mathrm{g}^{-1}$ in nonischemic myocardium. There was no significant difference in the blood flow values assessed with fluorescently or radioactively labeled microspheres.

The absolute intermethod errors are presented. 


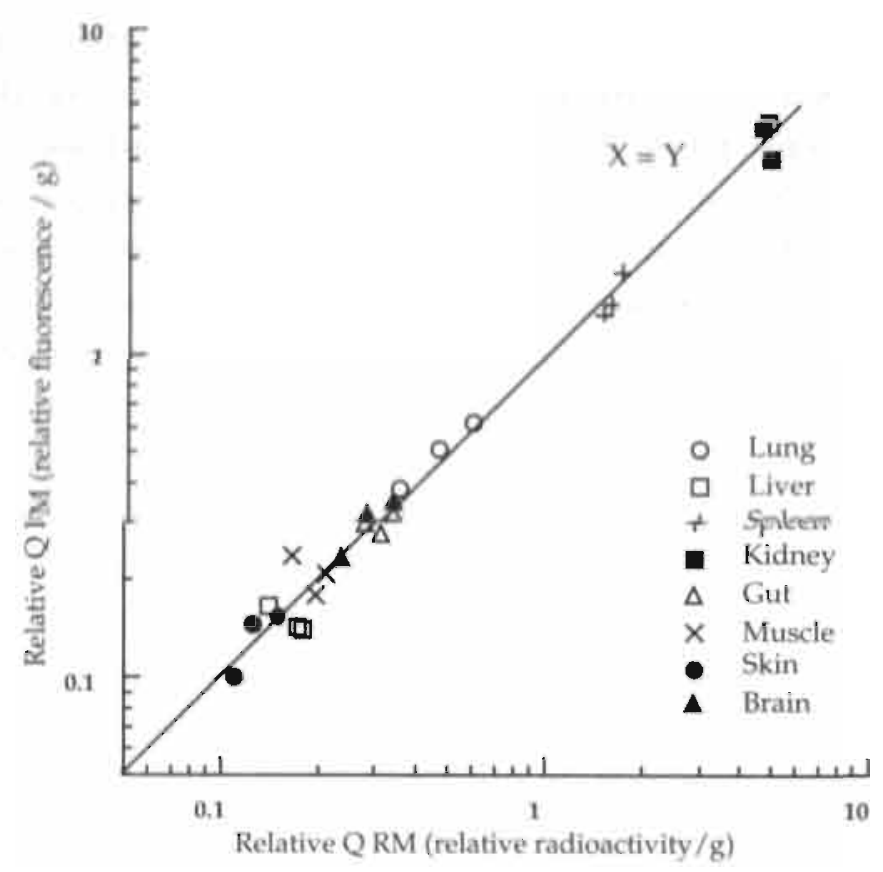

Fig 3.4 Intermethod eculuation of regional perfusion measurements for nonmyocardial organs as determined by simultaneous injection of fluorescently $(\overline{\mathrm{Q}} \mathrm{cm})$ and radionctively labeled microspheres $(\overline{\mathrm{Q}} \mathrm{m})$ for one experiment. Relatioc blood flow (expressed as the relative fluores-cence or radioactivity $\left.g^{-1}\right)$, is plotted on a logarithmic scale. Drawen line is line of unity. Regression equation for all organs together in this experiment is $y=0.995 x$ +0.004 and $r=0.991$. Absolute blood flow (derived from the radionctive microspheres only) in these organs ranged from 0.14 (skin) to $6.11 \mathrm{ml} \cdot \mathrm{min}^{2} \cdot \mathrm{g}^{2}$ (kidney).
There was no significant offset (mean differences, 0.00 and $0.02 \mathrm{ml} \cdot \mathrm{min}^{-1} \cdot$ sample $^{-1}$ ) and only a slight variation (SD of the mean difference, 0.07 and $0.15 \mathrm{ml} \cdot \mathrm{min}^{-1} \cdot$ sample $^{-1}$ ) between the two methods for ischemic and nonischemic myocardium, respectively. For ischemic and nonischemic myocardial samples fogether the relative intermethod error was $-1.90 \pm 11.15 \%$.

A good correlation ( $r=0.974$ ) was found between the endocardial-to-epicardial blood flow ratios as assessed with the fluarescent and radioactive microsphere methods as well (Fig. 3.3). The slope (0.91) and intercept (0.07) of the regression equation were statistically different from identity and origin, respectively. However, the regression equation was strongly influenced by one (unexplained) stray value (arrow, Fig. 3.3). If this point was omitted from the calculation of the regression line, regression improved to $r=0.985$, and the slope $(0.96)$ and the intercept $(0.034)$ were not statistically different from identity and origin, respectively. It was therefore concluded that fluorescent and radioactive microspheres estimated endocardial-toepicardial blood flow ratios equally well.

\section{Other organ perfusion studies}

The intermethod correlation for various non- 
myocardial tissues is shown in Fig. 3.4 (one experiment) and in Table 3.2 (pooled data for all experiments). For these organs the intermethod correlation was also good ( $r$ $=0.982$ ), whereas the intercept and the slope of the regression equation were not significantly different from 0 and 1 , respectively.

\section{DISCUSSION}

A new technique for sample digestion and isolation of fluorescent microspheres is described and validated. In the present study this time- and mone-saving, one vessel, sedimentation technique was suitable for myocardium as well as a variety of other organs. With the use of subsequent dye extraction and fluorimetry, reliable blood flow estimates can be obtained with up to 6 different dyes in the same experiment.

The accuracy of the blood flow measurements, expressed as the intermethod correlation, reported here $(r=0.985)$ is comparable to that found by others using direct extraction from lung tissue ( 5 fluorescent labels, $r=0.99$ ), or negative pressure filtration in myocardial $(r=0.95)$ and kidney samples ( 3 fluorescent labels, $r=0.96$ ), or protease tissue digestion and purification through sucrose gradients in normal and ischemic myocardium (3 fluorescent labels, $r=$ 0.99). ${ }^{2}$ Slopes and intercepts from the regression equations were not statistically different from 1 and 0 , respectively, for all methods. Also, the relative intermethod error for myocardium found in this study $(-1.90 \pm 11.15 \%)$ was also slightly, but not significantly, smaller than that found by Glenny and colleagues $(5.10 \pm 15.49 \%)$. The data obtained in the present study also show that the absolute intermethod error for ischemic myocardium $(0.00 \pm 0.04)$ is smaller than in nonischemic myocardium $(0.02 \pm 0.15)$. The accuracy of the fluorescent microsphere method in ischemic myocardium and in various other organs was excellent, indicating that the sample processing technique is reliable in organs with low perfusion and in organs that are normally hard to digest due to a high fat content (e.g., brain, skin, and liver).

An important practical limitation of the fluorescent or other nonradioactive microspheres is the time and costs involved in processing a sample. For the radioactive microsphere technique this time is relatively short, and the processing is easy. Time and costs involved in the processing of solid tissue samples varies for the different methods used (Table 3.5). With the sedimentation method, processing of a sample takes $6 \mathrm{~min}$, whereas in the 
vacuum filtration ${ }^{2}$ and the sucrose-cushion ${ }^{2}$ techniques 10 min and $\geq 9.5 \mathrm{~min}$ are involved, respectively. This means

Table 3.5: Time and costs of the sedimentation, filtration and sucrose-cushion methods

\begin{tabular}{|c|c|c|c|c|c|c|}
\hline \multirow[b]{2}{*}{ (C) } & \multicolumn{2}{|c|}{ Sedimentation (A) } & \multicolumn{2}{|c|}{ Filtration (B) } & \multicolumn{2}{|c|}{ Sucrose-Cushion } \\
\hline & & & & & & \\
\hline Processing & Time, & Cost, & Time, & Cost & Time, & Cost, \\
\hline Step & min & $\$$ & $\min$ & $\$$ & $\min$ & $\$$ \\
\hline Isolation & 2.9 & 0.05 & 6.9 & 0.45 & $>3$ & 0.05 \\
\hline Other & 2.0 & 0.01 & 2.0 & 0.01 & 2.0 & 5.72 \\
\hline Fluorescence & 1.1 & 0.11 & 1.1 & 0.06 & $* 4.5 / * 15$ & - \\
\hline Total & 6.0 & 0.17 & 10 & 0.52 & $9.5 / 20$ & 5.77 \\
\hline
\end{tabular}

Tabie 3.5 Estimation of time and costs (in US dollars) to process a sample (calculated as $1 / 50$ of the time or cost for a series of 50 samples) by the sedimentation techmique (this paper), the filtration technigue (Glenny of al.') and the sucrose-cushion fechnique (Austin et al.'). Isolation, sedimentation in centrifuge (A,C) or vacuum filtration (B); Other, numbering tubes, weighing samples, and preparing chemicals; Fluorescence, determination of fluorescence by spectroplotometry $(A, B)$ by manual counting with fluorescence microscopy (") or by fluorescence-activated cell sorter $(" *, C)$. Costs were related to cost of tubes (5 times reusable) $(A, B, C)$ and filters (B) for isolation and to chenicals I KOH (A,B) or proteinase/collagenase (Sigma) (C) for other and to 2-(2-ethoxyethoxy) ethyl acetate (Aldrich) (A,B) for fluorescence. Costs of laboratory personal or apparatus were not included. Time was not always subdivided into categories by authors; in B a total time and time for fluorescence was given, in C only an estimation of time for fluorescence was given. We estimated that time for other would not differ for different methods (2 min) and isolation in (C) would take at least 3 min. a reduction of $\geq 37 \%$ with the simplification described in the present study. Total cost for the sedimentation method amounts to $\$ 0.17$, and for the other methods to $\$ 0.52$ and $\$ 5.77$, respectively, which means that a cost reduction of $\geq 67 \%$ can be achieved with the sedimentation method. We therefore conclude, that the sedimentation method saves time and money compared with negative pressure filtration or counting the microspheres.

\section{Sources of error:}

As can be seen in Fig. 3.2, the radioactive microsphere method has small inherent errors, which have been well documented. ${ }^{3.59}$ The error is mainly determined by statistical variation in the distribution of the microspheres and is dependent on the number of microspheres in each tissue and reference sample. A minimum number of 400 microspheres per sample should guarantee a relative error of less than $5 \%$. In the present study, for each label the number of microspheres irijected $\left(3 \cdot 10^{6}\right.$ into left atrium) resulted in a mean of 1580 microspheres, ranging from 10 microspheres in small ischemic samples to 7500 microspheres in normoxic samples.

The results of the present study demonstrate that: the flow values obtained with the fluorescent microsphere 
technique correlate quite well with those obtained with the radioactive microsphere method and that the error made with the fluorescent microsohere technique is only slightly larger than the error made with the radioactive microspheres. This indicates that possible additional sources of error of the fluorescent microsphere technique are small. Such possible errors are incomplete isolation of microspheres from the samples and loss of microspheres or dye from microspheres during sample processing. Incomplete isolation of microspheres from tissue or blood would have resulted in underestimation and overestimation of organ blood flow, respectively, or in a greater variability in the flow values. The same holds for loss of microspheres or loss of dye during sample processing. Absence of loss of microspheres during sample processing was also demonstrated by the very low number of microspheres in the supernatant and the complete recovery of microspheres when these spheres were added as an internal reference. The absence of large systematic bias or large variability between the radioactive and fluorescent microsphere methods demonstrates that these possible sources of error are negligible compared to the error related to the number of microspheres per sample. In addition, the fact that a small, slightly variable amount $(<0.4 \mathrm{ml})$ of fluid (mainly water) remained in the test tubes after the final removal of supernatant does not seem to be a source of error. The presence of this small amount of supernatant together with a relatively large amount $(3 \mathrm{ml})$ of solvent induces only a small error, if any, because the volume of distribution of the highly lipophylic dyes in the solvent is about $100 \%$.

\section{Effects of microsphere density}

Rheological properties of microspheres can influence blood flow estimation. The diameter of microspheres has a significant influence on intraventricular and intrarenal blood flow distribution. ${ }^{60,13} 16$ There are no differences in diameter between radioactive and fluorescent microspheres, but there is a significant difference in specific gravity $(1.3 \mathrm{~g} / \mathrm{ml}$ for radioactive and $1.05 \mathrm{~g} / \mathrm{ml}$ for fluorescent microspheres). To the best of our knowledge no studies are available on the effect of the specific gravity of microspheres on the blood flow values obtained. Although the design of our study can not conclusively answer this question, comparison of endocardial-toepicardial blood flow ratios obtained with fluorescent and radioactive microspheres gives some insight into this aspect. Because of the close similarity between the 
endocardial-to-epicardial ratios as determined with fluorescent and radioactive microspheres found in this study (Fig، 3.3), it may be concluded that the rheological properties of both types of microspheres are not different, despite the difference in specific gravity. If more streaming had occurred with the heavier radioactive microspheres under normoxic circumstances, these microspheres would have been preferentially deposited in the endocardial layers, resulting in significantly higher endocardial-toepicardial blood flow ratios.

\section{Fluorescence technology}

As has be shown before spectrofluorometry provides a sensitive, highly repeatable measure that is linear with respect to the number of fluorescently labeled microspheres per sample. Repeatability of fluorescence measurements was highest when analyzing samples the same day $\left(C_{v}=0.2-1.1 \%\right)$. The repeatability decreased when the samples were analyzed on different days $\left(C_{v}=\right.$ $2-9 \%$ ) which has been observed before. Therefore, in the present study absolute blood flow calculations were only performed if fluorescence in tissue and the arterial reference sample was determined on the same day.

An advantage of fluorescent dyes over radio- isotopes is the miniml spectral spillover, being largest for crimson to red $(3.20)$. The actual overestimation of fluorescence of a dycbecause of spillover from another fluorescent dye is dpendent on 1) the percentage of spillover and 2) the fifference in fluorescence between dyes. For example, th overestimation of red (because of a spillover from crimso to red of $3.2 \%$ ) would be 7 and $35 \%$ whien the ratio of crimson to red fluorescence equals 2:1 and 10:1, respectively: In this study, in all samples the fluorescence of crimson was less $(20-50 \%)$ than the fluorescence of red, so the actual overestimation of red due to spillover from crimson was theoretically $<1.6 \%$. Spillover between the 6 different dyes used in this study did not affect the accuracy of the blood flow values obtained. This was demonstrated by the fact that blood flow values derived from fluorescence data corrected for spillover did not differ significantly from those not corrected for spillover.

It was observed that blue fluorescent microspheres cause a greater variability in the blood flow estimation than other dyes (Table 3). This could be explained by the varying background fluorescence of blue. The solvent used in this study [2-(2-ethoxyethoxy) ethyl acetate] has a significant intrinsic background in the blue excitation/ 
emission wavelength range. Moreover, Tween 80 dissolves in the solvent and has a significant background fluorescence in the blue wavelength range. To circumvent this problem, the final concentrations of Tween 80 were minimized, using water without Tween for the final washing step in the microsphere isolation procedure. Although the mean background fluorescence value was subtracted from the value of each sample, variation of background in individual samples may have contributed to a larger variability in blood flow estimation for the blue microspheres.

Therefore the present study shows that 5 fluorescent dyes can be used in the same experiment (the use of the blue label is less desirable) without the need for spectral spillover correction. When such correction is applied, at least one other fluorescent dye (green) can be used?

\section{Conclusions}

This study shows that 1) with autolysis and ethanolic $\mathrm{KOH}$ digestion, samples from blood and all kinds of organs can be easily digested, 2) all microspheres can be recovered from the samples by subsequent centrifugal sedimentation, 3 ) this easy method saves time and money compared with classical negative pressure filtration, 4) blood flow can be estimated, using up to six different fluorescent labels in the same experiment, with an accuracy similar to that of the radioactive microsphere technique in normally and hypoperfused myocardium and in various other organs, and that 5) endocardial-toepicardial blood flow ratios estimated with fluorescent microspheres equal those estimated with radioactive microspheres.

\section{REFERENCES}

1. Abel, E. L R. H Cooper and R. R. Beck. Use of fluorescent latex microspheres to measure coronary blood flow distribution. Circ. Shock 41: 156-161, 1993.

2. Austin, G. E., D. Martino-Salzman, A. G. Justic, A. C. Brooks, M. B. Tuvlin, R. L. Hunter and N.K. Thompson. Determination of regional nyocardial blood flow using fluorescent microspheres. An . ]. Cardiovasc. Pathol 4:352-357, 1993.

3. Austin, R. E. W. W. Hauck, G. S. Aldea, A. E. Flynn, D. L Coggins and J. I. E. Hoffman. Quantitating error in blood flow measurements with radioactive microspheres. Am. J. Physiol. 257: H280-H288, 1989.

4. Bland. I. M. and D. G. Aliman Statistical methods for assessing agreement between two methods of dinical measurement. The Lancet 307-310, 1986.

5. Buckberg, G. D., I. C. Luck, D. B. Payne, I. I. E. Hoffman, J. P. Archie and D. E. Fixler. Some sources of error in measering regional blood flow with radioactive nicrospheres. J. Appl. Physiol. 31: 598-604, 1971

6. Clausen, G., A. Kirkebo, I Tyssebotn, E. S. Ofjord and K. Aukland. Erroneous estimates of intrarenal blood flow' distribution in the dog with radiolabeled microspheres Acta Physiol Scand. $107 \cdot 385-387$, 1979

7. Glenny, R. W. S. Bernard and M. Brinkley. Validation of fluorescent-labeled microspheres for measurement of regional organ perfusion. J. Appl Physiol 74: 2585-2597, 1993

8. Heymann, M. A., B. D. Payne, I. L. E. Hoffman and A. M. Rudolph. Blood flow measurements with radionuclide-labeled microspheres. Prog. Cardiovasc. Dis, 20, 55-79, 1977 


\section{Chapter 3}

9. Nose, Y., T. Nakamura and M. Nakamura. The microsphere method facilitates statistical assessment of regional blood flow. Basic Res. Cardiol. 80: 417-429, 1985.

10. Ofiord, E. S. G. Clausen and K. Aukland. Skimming of microspheres in vitro: implications for measerment of intrarenal blood flow. Am. J. Physiol. 241: H342. H347, 1981.

11. Prinven, F. W., R. Alewijnse, G. J. Van der Vusse, R. T. I. Kruger, T van der Nagel and R. S. Reneman. Coronary artery stenosis controlled by distal perfusion pressure: description of the servo-system and time dependent changes in regional myocardial blood flow. Basic Res. Candiol. 82: 375-387, 1987.

12. Prinzen, F. W., T. Arts, G. I. Van der Vusse, W. A. Coumans and R.S. Reneman. Gradients in fiber shortening and metabolism across the ischemic left ventricular wall. Am. J. Physiol. 250: $1255-\mathrm{H} 264,1986$

13. Reneman, R. S. A. H. M. Jageneau, W Van Gerven, I. Dony and P Beimaert. The radioactive microsphere method for the assessment of regional myocardial blood flow after coronary artery occlusion. Pfluegers Arch. 353: 337-347, 1975.

14. Rudolph, A. M. and M. A. Heymann (1971). Measurement of flow in perfused oryans, using microsphere techniques, Karolinska Symposia IV 112-127.

15. Schosses, R., K.-E. Arfors and K. Messmer. MIC-11. A program for the determination of cardiac output, arterio-venous shunt and regional blood flow using the radioactive microspheres method. Comput. Progr. Biomed. 9; 19-39, 1979.

16. Utley, J., E. L. Carlson, J. I. E. Hoffman, H. M. Martinez and G. D. Buckberg. Total and regional myocardial blood flow measerments with $25 \mathrm{~m}, 15 \mathrm{~m}, 9 \mathrm{~m}$ and filtered 1-10m diameter microspheres and antipyrine in dogs and sheep. Circ. Res. 34: 391-$405,1974$. 


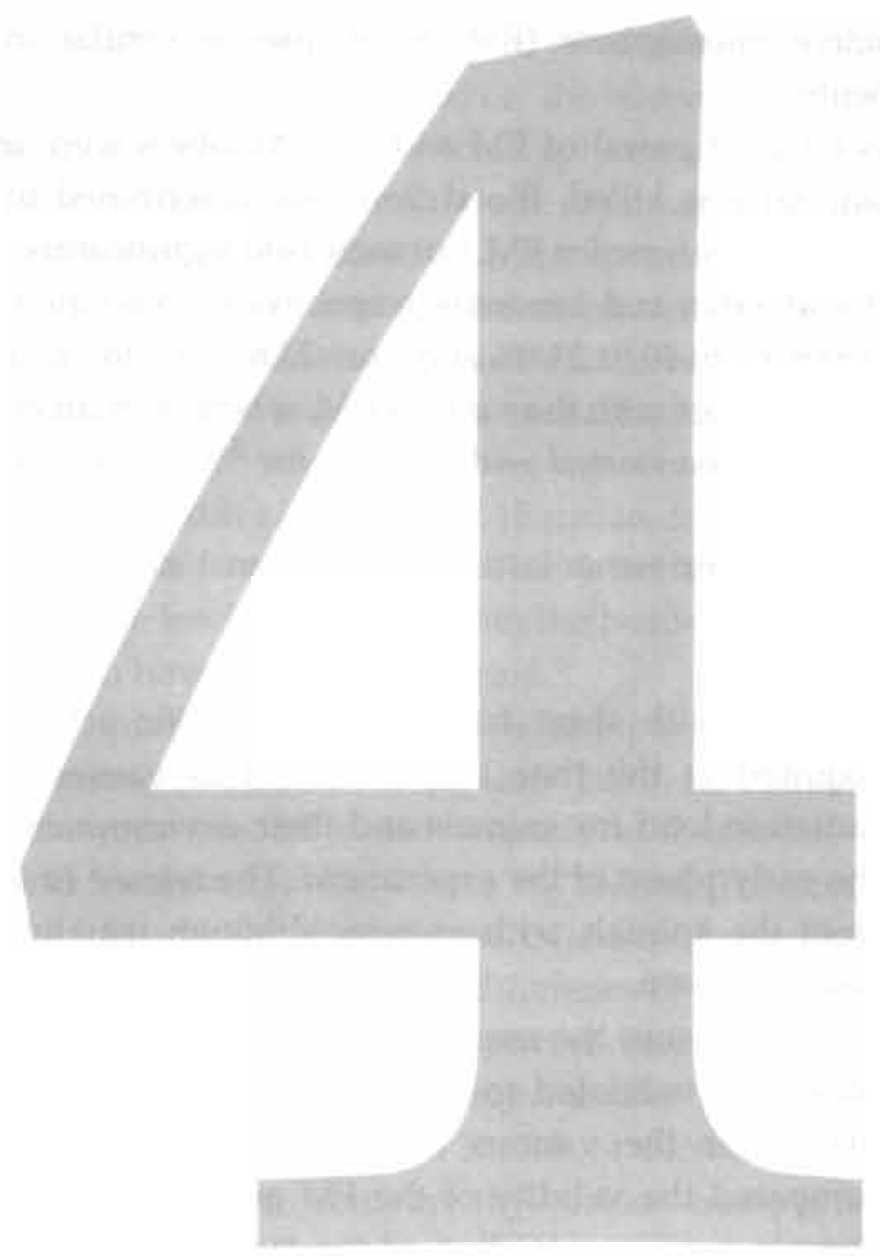

\section{FLUORESCENT MICROSPHERES ARE SUPERIOR TO RADIOACTIVE MICROSPHERES IN CHRONIC BLOOD FLOW MEASUREMENTS}

M.F.M. van Oosterhout', F.W. Prinzen', S. Sakudara2, R.W. Glerıny ${ }^{3}$, J.R.S. Hales ${ }^{2}$ 'Cardiovascular Research Institute Maastricht (CARIM), The Netherlands ${ }^{2}$ School of Physiology and Pharmacology of the University of New South Wales, Sydney NSW, Australia ${ }^{3}$ Departments of Medicine and of Physiology and Biophysics, University of Washington, Seattle, Washington 98195 , U.S.A.

Am.J.Physiol.275:H110-H115, 1998. 
Background - The accuracy of the fluorescent (FM) and radioactive microsphere (RM) techniques is similar in acute experiments but has not been established in chronic experiments.

Methods and Results - In the present study various combinations (at least pairs) of FM and/or RM labels were injected simultaneously between 2 months and 5 min before each animal was killed. Blood flow was determined in many organs. Intramethod mean difference and variation did not change over time for FM, but increased significantly for RM (from $1.8 \pm 1.4 \%$ to $25.6 \pm 21.8 \%$ and from $4.4 \pm 3.2 \%$ to $32.4 \pm 23.0 \%$, at $5 \mathrm{~min}$ and 2 months, respectively). Also the FM-RM intermethod mean difference and variation increased (from $-0.5 \pm 8.5 \%$ to $40.8 \pm 23.8 \%$ and from $23.6 \pm 4.6 \%$ to $71.8 \pm 34.3 \%$, respectively). After 2 months, blood flow estimations were 20-50\% lower with the various RM, whereas brain and liver blood flow values varied even more between isotopes. Underestimation started within 1 day for ${ }^{51} \mathrm{Cr}$ and within two weeks for ${ }^{141} \mathrm{Ce},{ }^{95} \mathrm{Nb}$ and ${ }^{85} \mathrm{Sr}$.

Conclusions - FM are superior to RM for blood flow determination in experiments lasting longer than 1 day, presumably because of leaching of isotopes from RM.

$\mathbf{T}$ he fluorescent microsphere technique has been demonstrated to be an excellent alternative for the radioactive microsphere technique in acute experiments. ${ }^{2.414}$ The accuracy of the FM technique is comparable to that of its radioactive counterpart, and, although the method is more time consuming, the lack of radiation for workers and the environment makes the fluorescent method safer and presents less legislative problems. The high costs of storage and disposal of waste are also avoided.

Nonradioactive microspheres may offer even greater advantages in chronic experiments. In the case of isotopes with short half-life high specific activities are required at the time of injection. This causes a larger radiation load for animals and their environment during the early phase of the experiment. The release of isotopes from the animals with excreta, although usually low, is also a concern.

Because the use of RM in chronic experiments has only been validated to a limited extent and because $\mathrm{IM}$ may offer the various benefits mentioned above, we compared the validity of the FM and RM techniques in chronic experiments. Two of the factors determining the 
accuracy of the microsphere method sen deserve special emphasis in chronic experiments, i.e. the absence of both leaching of the label from the microspheres and disappearance of microspheres from the site of entrapment. ${ }^{10}$ In a study on leaching of radioactive microspheres from the heart Consigny et al. ${ }^{3}$ showed that microspheres appearing in the venous circulation are almost exclusively smaller than $12 \mu \mathrm{m}$. A similar conclusion was reached by a study of Medvedev et al. ${ }^{12}$ who determined the content of ${ }^{46} \mathrm{Sc}$ labeled spheres with a diameter of $15 \mu \mathrm{m}$ in donor hearts and in lungs of the recipients. The latter study also indicated some leaching of ${ }^{46} \mathrm{Sc}$ from the beads. Losses of ${ }^{125}$ land ${ }^{113} \mathrm{~S}$ n have also been reported. ${ }^{7.9}$

The present experiments were performed in rabbits. Various combinations (at least pairs) of FM and RM labels were injected simultaneously at 2 months, 1 month and $5 \mathrm{~min}$ before sacrifice of the animal. The accuracy of the RM and FM methods was evaluated by quantification of the variation and mean difference of blood flow values between RM and FM and between different labels of RM as well as of FM in a large number of organs. Because RM lost label within 1 month, a second set of animals was studied to compare the various isotopes in experiments lasting 1, 7 and 14 days.

\section{METHODS}

\section{In vitro test on leaching}

To test potential leaching of fluorescent labels from $5 \mathrm{M}$, we took 20 samples of $1 \mathrm{ml}$ each from a stock solution containing a mixture of all FM used in the present study; the spheres were suspended in Hemaccel. Samples were stored in the dark for 3 days and for 2 months, either at $37^{\circ} \mathrm{C}$ (the temperature at which microspheres stay while the animal is still alive) or at $-20^{\circ} \mathrm{C}$ (the temperature at which blood samples are stored while the animal is still alive in the chronic situation and at which all samples were stored between gammacounting and processing for fluorimetry). At the end of the storage period the samples were processed like the tissue and blood samples and the fluorescence was determined (see below). Because the product information from the supplier states that decay of the fluorescent labels is small $(<1 \%$ after 6 months storage in the dark), any decrease in the dye content during the 2 months storage was assumed to be due to leaching of the dye from the beads during the storage.

\section{Experimental protocol}

The experiments were performed according to the Guiding Principles in the Care and Use of Animals. New Zealand white rabbits $(1.5-4 \mathrm{~kg})$ were used for this study. At each of the injection times the rabbits were anesthetized with pentobarbitone sodium (30 $\mathrm{mg} / \mathrm{kg}$ body weight) injected via a marginal ear vein. Also, for each injection catheters were placed in a femoral artery via the saphenous artery for withdrawal of a reference sample and into the 

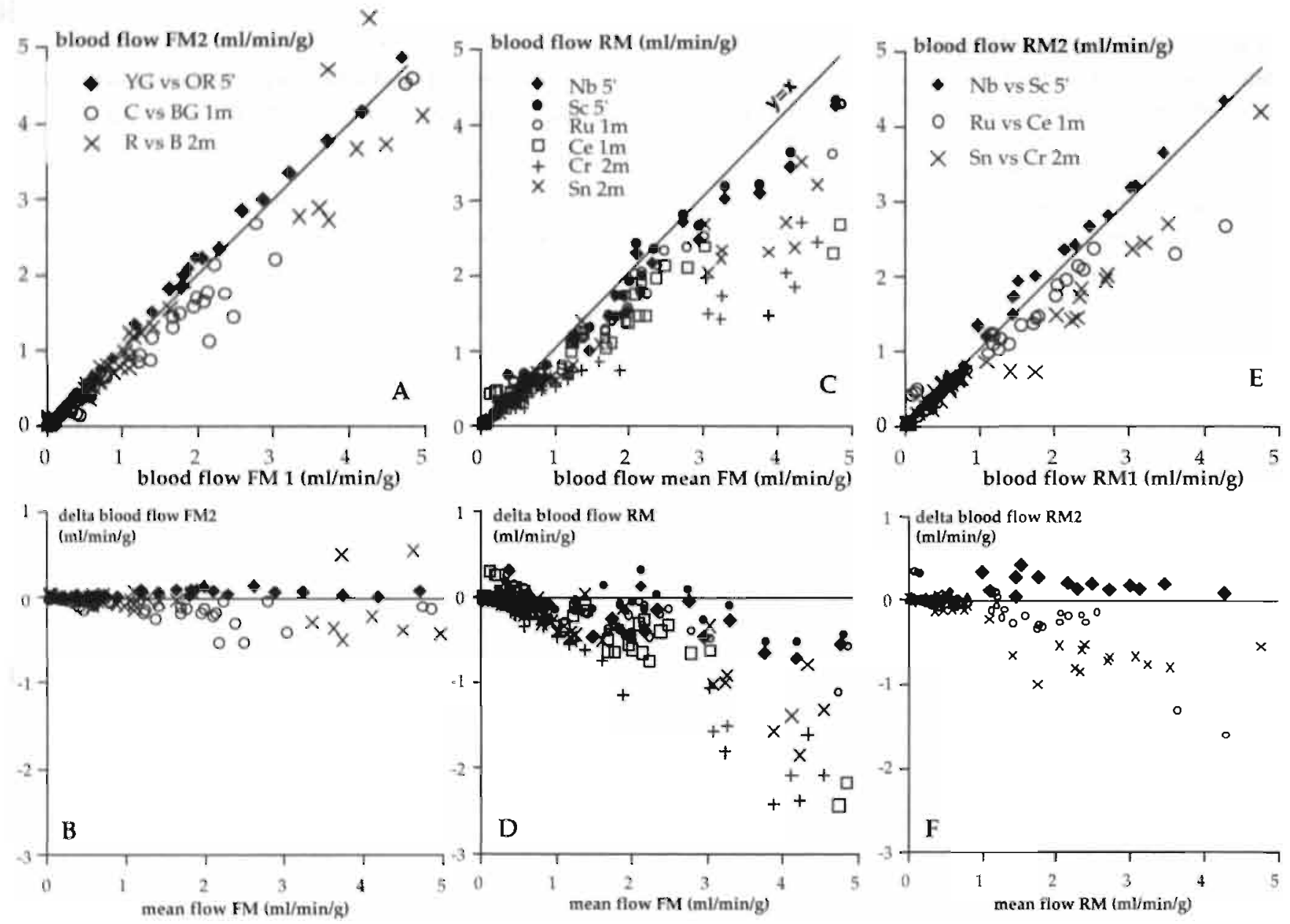
Figure 4.1: Regional blood floto as determined by simultaneous injection of pairs of (FM) and (RM) microspheres at three time intervals (2 months, 1 month and 5 min (acute) before animals twere killed) in one experiment. Top: regression plots for intramethod comparisons of FM (A) and $R M(E)$ and for intermethod comparison (C). In $A, x$-axis us. $y$-axis corresponds to yellowgreen (YG) vs. orange (Or); crimson (C) vs. blue-green (BG); and red ( $R$ ) vs, blue (B). In E, x-axis vs, y-axis cornesponds to Nb vs. Sc; Ru vs. Ce; and Sn vs. Cr. In B and F, x-axis corresponds, respectively, to mean flow of FM pair and RM pair, and $y$-axis corresponds, respectively, to change ( $\Delta)$ in blood flow of FM pair and of RM pair. In D, $x$-axis corresponds to mean flow of all FM labels used. Bottom; absolute ernors as a function of mean blood flow. Data and symbols of $B, D$, and $F$ correspond to regression plots of $A, C$, and E, respectively. Measurements are at 5 min (filled strmbols), 1 month (open symbols), and 2 months before animals were killed (t and $X$ ). In this particular experiment some samples, obtained from heart and lungs showed underestimation by B (2 months) and BG (1 month) compared with $R$ and C. respectively. Howewer, overall the slope and intercept of the regression equations were not statistically different from 7 and 0 , respectively. RM-RM intramethod variation increased with time, which becomes especially clear in F. The two outliers for Ce (see C-F) are from the two kidney samples in this experiment.

it can be seen that the intramethod variability (mean difference and variation) for the FM increased slightly over time; predominantly due to relatively low values for $B$ and BG in samples from heart and lungs in this particular experiment. Nevertheless, slope and intercepts of the regression equations for FM intramethod comparison were not significantly different from unity and zero, respectively $(\mathrm{y}=1.03 \mathrm{x}+0.01 \quad(\mathrm{r}=0.96, \mathrm{SEE}=0.06)$ after $5 \mathrm{~min}, \mathrm{y}=0.89 \mathrm{x}-0.07(\mathrm{r}=0.90, \mathrm{SEE}=0.20)$ after 1 month and $y=0.91 x+0.06(r=0.88, S E E=0.34)$ after 2 months $)$. For the whole group of experiments FM-FM intramethod variation did not change significantly over time (Table 4.2). Also, there was no systematic mean difference for any of the FM labels at any time point.

Figure 4.1, E and F, shows an increase in the intramethod variability for RM over time. Regression equations for RM intramethod comparison were $y=1.05 x+0.01$ $(\mathrm{r}=0.97, \mathrm{SEE}=0.08)$ after $5 \mathrm{~min}, \mathrm{y}=0.72 \mathrm{x}+0.13(\mathrm{r}=0.91$, $\mathrm{SEE}=0.19)$ after 1 month and $y=0.84 x-0.02 \quad(r=0.93$, $\mathrm{SEE}=0.21$ ) after 2 months. After 1 month the slope was significantly lower than unity. For the whole group the increase in RM-RM intra-method variation was statistically significant at 2 months (Table 4.2).

In the experiment shown in Figure 4.1 the intermethod mean difference increased at 1 and 2 months (Figures 4.1C and 4.1D). This was due to a decrease of the slope of the regression equation for the FM-RM comparisons from 0.86 and 0.92 at 5 min (N.S. from 1) to 0.81 and 0.57 after 1 month and 0.49 and 0.57 after 2 months (all significantly lower than unity). Intercepts were not significantly different from zero. For all experiments only the increase in mean difference at 2 months reached the level 
Table 4.2: Relative mean difference and variation within and between FM and RM blood flow determination by injection of microspheres at various time intervals before animal death.

\begin{tabular}{|lrcc|}
\hline & Acute & 1 month & 2 months \\
\hline Mean difference, \% & & & \\
FM-FM & $5.0 \pm 3.9$ & $6.1 \pm 3.7$ & $5.9 \pm 5.7$ \\
FM-RM & $-0.5 \pm 8.5$ & $1.9 \pm 10.3$ & $40.8 \pm 23.5 *$ \\
RM-RM & $1.8 \pm 1.4$ & $13.6 \pm 10.6$ & $25.6 \pm 21.8^{*}$ \\
Variation, \% & & & \\
FM-FM & $10.4 \pm 6.2$ & $9.5 \pm 4.5$ & $13.5 \pm 9.7$ \\
FM-RM & $23.6 \pm 4.6$ & $31.8 \pm 16.1$ & $71.8 \pm 34.3 *$ \\
RM-RM & $4.4 \pm 3.2$ & $28.2 \pm 21.8$ & $32.4 \pm 23.0^{*}$ \\
\hline
\end{tabular}

Table4.2 Mean difference and variation values are means $\pm S D$, expressed as $\%$ of the mean value. The relative mean difference presented is comparable to slope of regression line through data points in figure 1. For calculation of mean difference and variation, see METHODS. Animals were killed 5-(acute), 1 month, or 2 months after injection of spheres. FM, fluorescent microspheres, $R M$, readioactive microspheres, " $P<0.05$ vs. acute; $t P<0.05$ vs. I month, according to 1 -way ANOVA and post hoc testing.

of significance. Because the inter-method mean difference is defined as $\overline{\mathrm{Q}}_{\mathrm{FM}}-\overline{\mathrm{Q}}_{\mathrm{RM}}$ (see METHODS), the positive intermethod mean difference indicates lower blood flow estimations by RM than by FM. The increase in intramethod mean difference at 1 month was not significant because of higher RM blood flow estimates in one experiment. In this particular experiment $\mathrm{Cr}$ proved to result in higher flow values than the other RM and FM labels for several organs. Intermethod variation significantly increased at 1 and 2 months (Table 4.2).

After 2 months blood flow values from RM were on average $40 \%$ lower than those obtained with FM (Table 4.2). This difference was smallest for $\mathrm{Sn}, \mathrm{Ce}$ and $\mathrm{Ru}$ (Figure 4.2). In the case of $\mathrm{Ce}$ and $\mathrm{Ru}$, however, this was due to disproportionally high values in brain ( $\mathrm{Ru}$ and $\mathrm{Ce}$ ) and

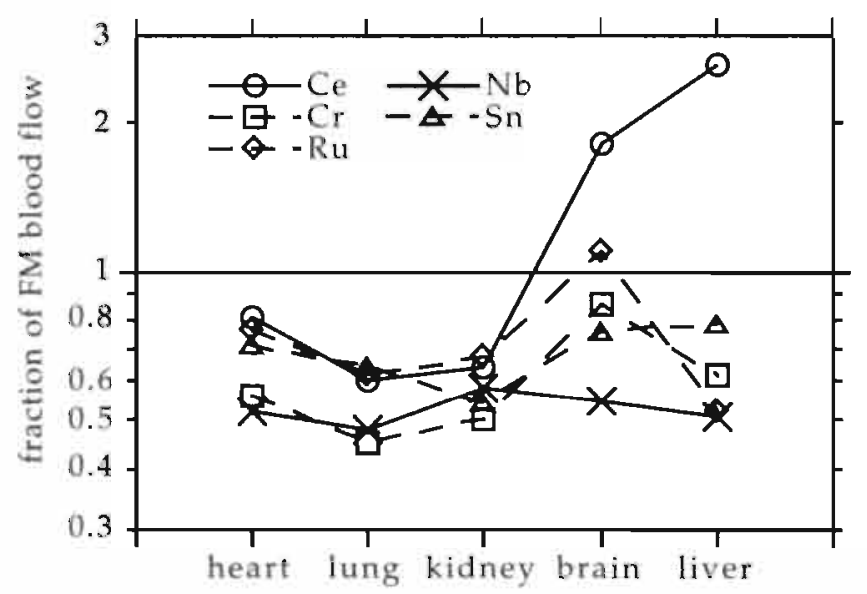

Figure 4.2 Error in blood flow by RM, specified per organ. RM blood flow values are expressed as a fraction of FM blood flow (mean of the two simultaneously injected labels) 2. months before animal was killed. Note log scale on vertical axis. Each data point is average of 3-5 tissue samples from organs of 1-2 animals. 
liver (Ce). In the latter organ Ce blood flow was more than twice the FM values (Figure 4.2). Blood flow values obtained with $\mathrm{Cr}$ and $\mathrm{Nb}$ were approximately $50 \%$ lower than those obtained with FM. This difference was similar in all organs for $\mathrm{Nb}$, whereas brain flow values obtained with $\mathrm{Cr}$ were closer to the FM values (Figure 4.2). These interisotope and interorgan differences in RM blood flow

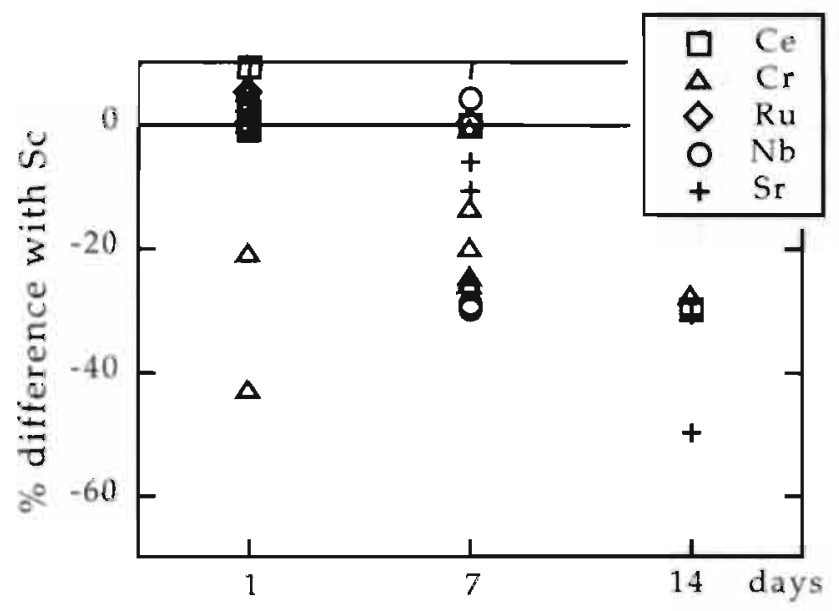

Figure 4.3 Mean differences in blood flow estimation over all organs by RM at 1,7 and 14 days before animal was killed. Each symbol denotes average underestimation by a label from all 40-60 samples in an individual experiment. estimations explain the high FM-RM intermethod variability (Table 4.2). At the time of death significant amounts of $\mathrm{Ce}, \mathrm{Cr}, \mathrm{Ru}$ and $\mathrm{Nb}$ were found in the urine and faeces of some animals. Blood flow estimations from the various FM labels were similar in all organs (data not shown).

When RM were injected 1 day before the animal was killed, all labels except $\mathrm{Cr}$ gave similar blood flow values (Figure 4.3). Injection of RM 7 days before the animal was killed resulted in 15-30\% underestimation of blood flow by $\mathrm{Cr}, \mathrm{Sr}$ and $\mathrm{Nb}$ compared with $\mathrm{Sc}$. During the experiments lasting 14 days a slightly more pronounced underestimation was observed for $\mathrm{Sr}, \mathrm{Cr}$ and $\mathrm{Ce}$ (Figure 4.3).

\section{DISCUSSION}

This study demonstrates that in chronic experiments of up to 2 months blood flow estimation by FM is reproducible, whereas RM lead to variable results and usually lower values than obtained with FM. Therefore, FM are to be preferred for blood flow measurements in experiments lasting longer than 1 day.

The mean difference and variation between any two fluorescent labels do not significantly change within 2 
months. This indicates that FM perform virtually as well in chronic as in acute studies, and that FM are suitable to follow changes in absolute blood flow over time, at least for 2 months. The reliability of FM is probably due to stability of labels. This idea is supported by the in vitro studies.

The present study can not exclude dislodgment of spheres over time. Loss of spheres from a tissue is likely to be reflected in a relatively high lung blood flow value as the spheres are trapped by the lungs ot In the present study, however, comparison of absolute lung flows between various injection times is hampered by variable conditions between injections, like ambient temperature, which may influence shunt flow. Although absence of dislodgment can not be proven in the present study, three previous studies virtually exclude the likelihood of dislodgment of microspheres in chronic studies. Hales and Cliff reported that losses from the rabbit ear or thoracic tissue had stopped within a few minutes after injection and were not detectable for up to 8 weeks. Consigny et al. ${ }^{3}$ demonstrated that over a period of 5 weeks part of the microspheres with a diameter $<12 \mu \mathrm{m}$ disappear from the myocardium, but hardly any dislodging of microspheres with diameters $\geq 15 \mu \mathrm{m}$ occurs. Medvedev et al. ${ }^{12}$ counted activity of hearts fron donor rats, which had previously been injected with Sc-labeled RM. The hearts were retrogradely perfused by attaching the ascending aorta to the abdominal aorta of the recipient animals. Within 4 weeks these investigators did not find accumulation of activity in the lungs of the recipient animal. ${ }^{12}$

The leaching of blue-green and crimson at $37^{\circ} \mathrm{C}$ in vitro may, in theory, lead to underestimation of tissue blood flows determined with these labels, since FM in the tissues remain at this temperature while the related reference blood samples were stored at $-20^{\circ} \mathrm{C}$. Figure $4.1 \mathrm{~A}$ and $B$, shows some examples of increased intramethod variability. However, in vitro leaching was relatively small (10\% in 2 months) compared to the errors observed with the RM method, and in vivo the increase in FM intramethod variability over time was not statistically significant (Table 4.2). Red appeared to be leached from the spheres at a similar rate at $-20^{\circ} \mathrm{C}$ and $37^{\circ} \mathrm{C}$. Therefore, this leaching would not result in a decline of blood flow, calculated with the reference method.

The generally limited extent of dislodgment of 15 $\mu \mathrm{m}$ diameter microspheres in general in combination with the minimal leaching of label from FM indicate that absolute blood flows can be determined accurately with FM in 
chronic experiments. The generally lower and more variable blood flow estimations by RM in chronic experiments, therefore, indicates poor performance of RM under these circumstances. While on average RM underestimate blood flow by up to $40 \%$ as compared to FM, considerable differences are found between isotopes and between organs. This can be observed from the intermethod variation at 2 months, which is considerably larger than both intramethod variations. $\mathrm{Cr}$, $\mathrm{Sr}$ and $\mathrm{Nb}$ underestimated blood flow most, $\mathrm{Cr}$ already within 1 day (Figure 4.3). Although underestimation by $\mathrm{Ce}$ and $\mathrm{Ru}$ appeared to be less compared with other isotopes, this observation is, in part, due to selective overestimation of blood flow in organs like the brain and the liver.

This variability in blood flow estimation by RM is most likely not caused by dislodgment of the spheres, since in that case blood flow to the lungs should be higher for RM than for FM. Instead, lung flows were underestimated by RM as much as other organs like the heart. Therefore, the most likely explanation is the loss of radioactive label from the beads. While loss of activity from the beads in most organs can explain the underestimation of tissue blood flow, selective binding of isotopes in some organs can explain overestimation, as for example, by $\mathrm{Ce}$ in the liver and $\mathrm{Ce}$ and $\mathrm{Ru}$ in the brain.

The inter-organ variation in leaching of the isotopes suggests that leaching is dependent on the environment. This environment is different in the various organs, since microspheres move to the interstitium within 1-3 weeks, ${ }^{, 3}$ thus becoming subject to the chemical environment of each particular organ. In the present study leaching of label is also suggested by the presence of $\mathrm{Ce}$, $\mathrm{Cr}, \mathrm{Ru}$ and $\mathrm{Nb}$ in urine and faeces at the time some animals were killed. The data of the present study also indicate that the leaching process is different between isotopes. Hales et al. attributed falsely high blood flow values for baboon liver obtained with $\mathrm{Sn}$ (manufactured by $3 \mathrm{M}$ ) to minute losses of that label from tissues in general and uptake in the liver. Similarly, ${ }^{125} \mathrm{I}$ appeared to be lost from spheres entrapped in the kidney, gastro intestinal tract and bone and taken up by thyroid and fat in sheep. These observations suggest that coating and/or chemical binding to the resin of the bead is of variable efficiency.

The present results are not in contradiction with those of Consigny et al., ${ }^{3}$ who studied the loss of microspheres from the myocardium. These investigators determined the chronic loss of microspheres from the 
myocardium from the radioactivity counted within and outside the heart rather than counting microspheres. In this approach loss of label from spheres will not be observed of loss is equal in and outside the heart.

The large variability in blood flow estimations between the various radioactive labels, as well as the increasing underestimation of flow with time, starting within a week after injection of the spheres, makes blood flow data from RM in chronic experiments unreliable. Although the inter-isotope variation was limited to $30 \%$ in organs like heart, lungs and kidneys, variation in the brain amounted as much as a factor 3 .

This poor performance of RM stands in contrast to the good correlation between RM and FM blood flow estimation in acute experiments ( $<1$ day), as has also been demonstrated in previous studies. ${ }^{4.14}$ Actually, the accuracy of the RM was slightly better than that of the FM, although in previous studies the accuracies of both methods were found to be similar. This may be due to the fact that in the present study determination of radioactivity was performed soon after the animal was killed, whereas the fluorescence measurements were performed after storage of the samples for up to a year. The in vitro studies show that long-term storage of samples at $-20^{\circ} \mathrm{C}$ may cause some leaching or destuction of spheres.

\section{Conclusions}

In chronic annal experiments the FM method is superior to the RM nethod, presumably because after periods of 1 day andlonger several isotopes are leached from spheres in the issue and may accumulate in other tissues. The accuracyof organ blood flow determination with FM does not leteriorate within 2 months. This advantage in the accuracy of blood flow measurements comes in addition to other advantages of FM, like lack of radiation and minimal decay of the label over time.

\section{REFERENCES}

1. Bland, J. M., and D. G. Altman. Statistical methods for assessing agreement between two methods of clinical measurement. Lancet : 307-310, 1986.

2. Chien, G. L., C. G. Anselone, R. F. Davis, andi D. M. Van Winkle. Fluorescent vs. radioactive microsphere measurement of regional myocardial blood flow. Candiovasc. Res. 30:405-412, 1995.

3. Consigny, P. M. E. D. Verrier, B. D. Payne, G. Edelist I. Jester, R. W. Baer, G. I Vlahakes, and J. I. E. Hoffman. Acute and chronic microsphere loss from canine ventricular myocardium. Am. J. Physiol. 242: H392-H404, 1982.

4. Glenny, R. W., S. Bernard, and M. Brinkley. Validation of fluorescent-labeled microspheres for measurement of regional organ perfusion. J. Appl. Physiol. 74: 2585-2597, 1993.

5. Hales, J. R. S. Practical considerations in the use of microspheres for evaluation of cardiovascular and thermoregulatory interactions. Biomed. Thermol. 13: 127-145, 1994.

6. Hales, I. R. S. Radioactive microsphere measurement of cardiac output and regional tissue blood flow in the sheep. Pfluegers Arch. 344: 119-132, 1973. 
7. Hales, J. R. S., J. W. Bennett, and A. A. Fawcett Effects of acute cold exposure on the distribution of cardiac output in the sheep. Pflugers Anch. 366: 153-157, 1976.

8. Hales, J. R. S., and W. J. Cliff. Direct observations of the behaviour of

microspheres in microvasculature. Bibl. Anat. 15:87-91, 1977.

9. Hales, J. R. S. R. B. King, and A. A. Fawcett. Observations on the validity of using NEN-Trac microspheres for measuring organ blood flow. Pfuegers Arch. 379: 295$296,1979$.

10. Heymann, M. A., B. D. Payne, J. I. E. Hoffman, and A. M. Rudolph. Blood flow measurements with radionuclide-labeled microspheres. Prog. Cardiovasc. Dis. 20: $55-79,1977$

11. Kaihara, S., P. D. Van Heerden, T. Migita, and H. N. Wagner. Measurement of distribution of cardiac output. I. Appl. Physiol. 25: 696-700, 1968

12. Medvedev, O. S., E. R. Martynova, R. S. Akchurin, and V. Y. Khalatov. Experimental estimation of chronic microsphere loss from the rat myocardium. Biull. Eksp. Biol. Med. 103: 8-10, 1987.

13. Prinzen, E. W., and R. W. Glenny. Developments in non-radioactive microsphere techniques for blood flow measurement. Cardiovasc. Res. 28; 1467-1475, 1994.

14. Van Oosterhout, M. F. M. H. M. M. Willigers, R. S. Reneman, and F. W. Prinzen. Validation of fluorescent microsphere technique for measurement of organ perfusion with improved sample processing method. Am. J. Physiol. 269: H725H733, 1995 . 


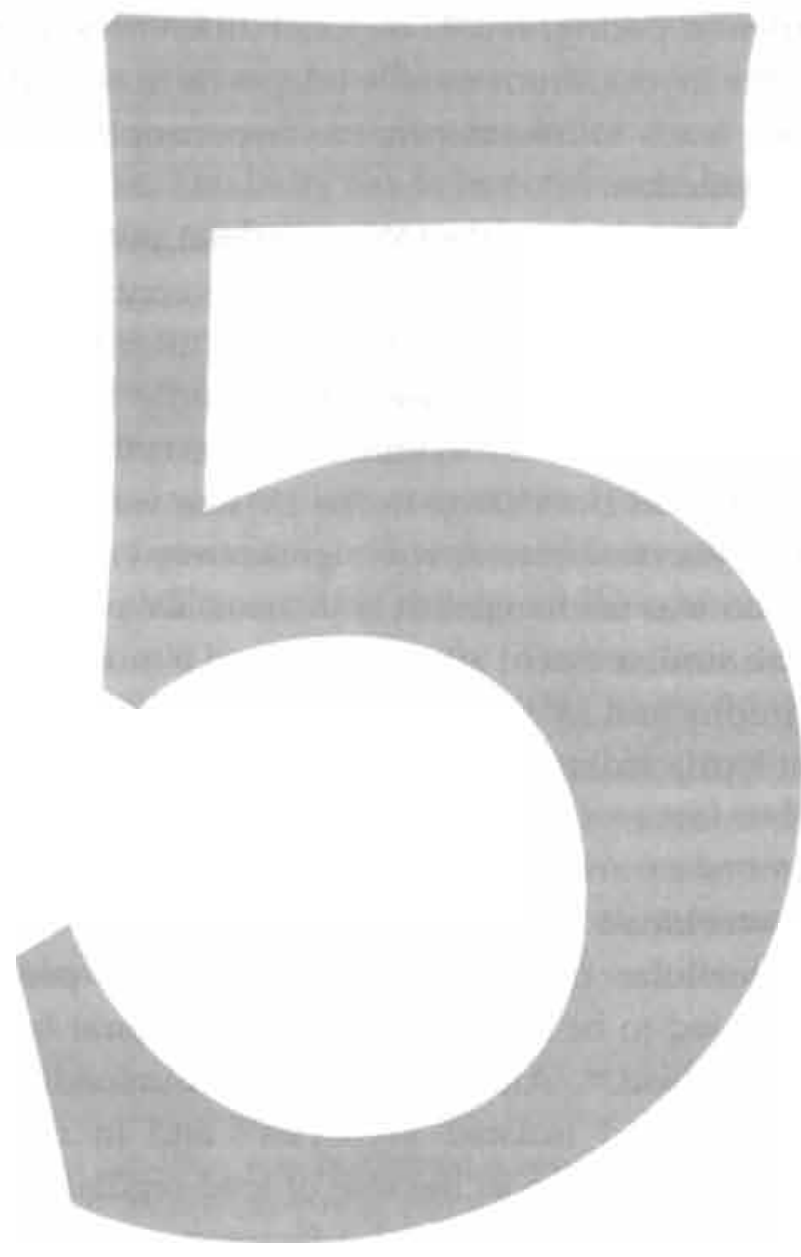

\section{ASYNCHRONOUS ELECTRICAL ACTIVATION INDUCES ASYMMETRICAL HYPERTROPHY OF THE LEFT VENTRICULAR WALL}

M.F.M. van Oosterhout, F.W. Prinzen, T. Arts, J.J. Schreuder, W.Y.R. Vanagt, J.P.M. C'leutjens, R.S. Reneman

Cardiovascular Research Institute Maastricht (CARIM), The Netherlands

(Circulation. 1998;98:588-595) 
Background - Asynchronous electrical activation, induced by ventricular pacing, causes regional differences in workload, which is lower in early- than in late-activated regions. Because the myocardium usually adapts its mass and structure to altered workload, we investigated whether ventricular pacing leads to inhomogeneous hypertrophy and whether such adaptation, if any, affects global left ventricular (LV) pump function.

Methods and Results -Eight dogs were paced at physiological heart rate for six months (AV sequential, AV interval 25 ms, ventricular electrode at the base of the LV free wall). Five dogs were sham operated and served as controls. Ventricular pacing increased QRS duration from $47.2 \pm 10.6$ to $113 \pm 16.5 \mathrm{~ms}$ acutely and to $133.8 \pm 25.2 \mathrm{~ms}$ after 6 months. 2Dechocardiographic measurements showed that LV cavity and wall volume increased significantly by $27 \pm 15 \%$ and $15 \pm 17 \%$, respectively. The early-activated LV free wall became significantly $(17 \pm 17 \%)$ thinner, whereas the late-activated septum thickened significantly $(23 \pm 12 \%)$. Calculated sector volume did not change in the LV free wall but increased significantly in the septum by $39 \pm 13 \%$. In paced animals, cardiomyocyte diameter was significantly $(18 \pm 7 \%)$ larger in septum than in LV free wall, whereas myocardial collagen fraction was unchanged in both areas. LV pressure-volume analysis showed that ventricular pacing reduced LV function to a similar extent after 15 min and 6 months of pacing.

Conclusions - Asynchronous activation induces asymmetric hypertrophy and LV dilatation. Cardiac pump function is not affected by the adaptational processes. These data indicate that local cardiac load regulates local cardiac mass of both myocytes and collagen.

V entricular pacing causes asynchronous electrical activation of the ventricles. In previous canine studies, ${ }^{23}$ we have shown that ventricular pacing decreases fiber shortening, contractile work, myocardial blood flow, and oxygen consumption in early-activated regions and increases these parameters in late-activated regions. The ventricular wall is known to adapt to changes in workload by changing cardiomyocyte size and extracellular matrix composition. These processes are supposed to be regulated by neurohumoral factors ${ }^{4.5}$ and cardiac load. ${ }^{\text {os }}$ Although studies on unloaded papillary muscles $^{6}$ and isolated myocytes ${ }^{78}$ and in mathematical simulations support the role of load-regulated growth, it is unknown whether local differences in workload, as in 
cardiac pacing, result in regional differences in myocardial mass. Neither is it known whether such an asymmetrical hypertrophy, if any, results in changes in left ventricular (LV) performance. The latter has to be considered because ventricular pacing reduces ventricular pump function acutely. ${ }^{1210-12}$

It was the aim of the present study to investigate the effect of asynchronous electrical activation of the LV on regional geometry and microscopic structure of the LV wall and on global ventricular geometry and performance. To this end, LV dimensions and regional ventricular wall geometry were determined by means of 2D-echocardiography at various time intervals during long-term ventricular pacing (PACE-group) or during sinus rhythm (SHAM-group). LV function, including LV pressurevolume analysis, was assessed at the beginning and end of the 6-month experimental protocol. Collagen content and myocyte dimensions were determined post mortem in tissue sections from the LV wall.

\section{METHODS}

Animal handling was performed according to the Dutch Law on Animal Experimentation (WOD) and The European Directive for the Protection of Vertebrate: Animals used for
Experimental and other Scientific Purposes (86/609/EU). The protocol was approved by the Animal Experimental Committee of the University of Maastricht.

\section{Implantation procedure}

Thirteen adult dogs were premedicated by an intramuscular injection of acepromazine $0.2 \mathrm{mg} / \mathrm{kg}$, atropine 0.1 $\mathrm{mg} / \mathrm{kg}$ and oxycodon $2 \mathrm{mg} / \mathrm{kg}$. Anesthesia was induced with Thiopental $15 \mathrm{mg} / \mathrm{kg}$ IV and maintained by ventilation with Halothane $(0.75-1.5 \%)$ in a $1: 2$ mixture of $\mathrm{O} 2$ and $\mathrm{N} 2 \mathrm{O}$. The ECG was recorded from the limb leads.

During sterile surgery a Medtronic CapSure sp 4423 lead was positioned into the right atrium and a Medtronic $4951 \mathrm{M}$ unipolar lead was inserted with its fishhook tip into the epicardium of the free wall of the LV, $1 \mathrm{~cm}$ below the base. This site was chosen because with this electrode position both early- and late-activated regions could be visualized in one echocardiographic cross section. In 8 dogs (PAC.E group, $28.9 \pm 9.5 \mathrm{~kg}$ ) a pacemaker (Medtronic Synergist H7027, H7071, Elite Il or Thera DR 7941) was implanted. In 5 dogs (SHAM group, $24.4 \pm 2.3 \mathrm{~kg}$, not significantly different from PACE group) no pacemaker was implanted, but, for assessment of the acute effects of pacing the leads were lemporarily connected to a pacemaker.

After closure of the pacemaker pocket and the thorax, LV cavity and ascending aortic pressure were measured with a dual tip micromanometer catheter (Sentron, Roden, The Netherlands) and cardiac output was measured by thermodilution. $\mathrm{LV}$ cavity volume was measured by use of a 12-electrode dual-field conductance catheter (7F, Sentron), advanced into the LV via the left carotid 
artery, connected with a Leycom Sigma 5DF signal conditioner processor (CardioDynamics). Parallel' conductance was estimated by injection of $5 \mathrm{ml}$ of hypertonic saline $(8 \%)$ into the pulmonary artery." Ventricular function was estimated from the slope and infercept of the end-systolic pressure-volume relation. Preload reduction, necessary to derive these values, was induced by inflating a balloon in the inferior caval vein.

Hemodynamics and ECG recordings were made under baseline conditions and 15 minutes after ventricular pacing was initiated. Hemodynamic signals were digitized with 12 bits at 200 $\mathrm{Hz}$ by use of a DASH 16 G2 A/D converter and stored on a personal computer for further off-line analysis.

\section{Pacing protocol}

In the PACE group, ventricular pacing was started approximately two weeks after implantation, when the dogs had fully recovered from surgery. The heart was stimulated at its own rhythm by AV sequential pacing (DDD-mode, upper rate 175 beats/min). The A-V stimulation interval was $25 \mathrm{~ms}$ to ensure complete ventricular capture. Proper pacemaker function and pacing thresholds were checked regularly and adjusted when necessary.

\section{Echocardiographic follow-up}

Two-dimensional echocardiographic images of the LV were made by means of a Hewlett Packard ultrasound system (77020A) with a $3.5 \mathrm{MHz}$ transducer $(21206 \mathrm{~A})$ and were recorded on Super VHS video-tape. Recordings were made at 0, 0.5, 1, 2, 3, 4, 5 and 6 months after onset of pacing in the paced animals and at 0 , 3 and 6 months in the sham animals while they were lying; on their right side. Animals were sedated with a mixture of acepromazine $(0.2 \mathrm{mg} / \mathrm{kg})$ and oxycodone $(1.2 \mathrm{mg} / \mathrm{kg})$. Long-axis images were made as well as parasternal short-axis cross-sectional images, taking care that the LV appeared as circular as possible and that the tip of the papillary muscles and the pacing lead were visible.

\section{Terminal procedure}

After 6 months, the dogs were operated again, using the same anesthetic and catheter-implantation procedures. Hemodynamic measurements (see above) were performed with the pacemaker still functioning and 15 min after the pacemaker had been switched off.

After these measurements were taken, the heart was arrested in diastole by perfusion with ice-cold $\mathrm{CdCl}_{2}(0.1 \mathrm{M})$. The heart was quickly removed, and the left ventricle was weighed. For histological analysis, a transmural tissue block was taken from each heart from the LV free wall, at or near the pacing site, and one from the septum, opposite to that site. These blocks were immersion fixed in phosphate-buffered formalin $10 \%$ and embedded in paraffin. Morphometry was performed with a Quantimed 570 image analyzer (Leica, Cambridge, UK). In a $5 \mu \mathrm{m}$ thick section, stained with a modification of the Azan technique," myocyte diameter and area were determined from 100 myocytes for each section by use of a final magnification of $X 400$. Only those myocytes in which the nucleus; was centrally located within the cell were digitized and analyzed to ensure that the short axis of the myocyte was perpendicular to the microscope objective. ${ }^{15}$ In a $6 \mu \mathrm{m}$ thick section stained with Sirius Red" (Polysciences, Warrington, PA) the collagen-positive area: was determined in 45 fielas (magnification 
X250), excluding epicardial and endocardial as well as perivascular areas. ${ }^{\text {C }}$ Collagen content was expressed as fraction of the total area examined. The sample size for myocyte diameter and collagen assay was chosen on the basis of a progressive means test, indicating that with the sample sizes used the mean values were within $3 \%$ and $8 \%$, respectively, of the mean value obtained by use of a larger sample.

All histological measurements were performed while the observer was blinded for the experimental group and the wall sector the tissue was taken from.

\section{Hemodynamic data analysis}

Hemodynamic data were analyzed off-line using software developed in our laboratory. The dedicated data acquisition and analysis software package CONDUCT-PC (CardioDynamics) was applied for conductance catheter-related data analysis. We calculated absolute LV cavity volumes, by calibrating systolic conductance changes to stroke volume as determined from thermodilution cardiac output and heart rate. ${ }^{13}$ The time constant of monoexponential LV pressure decline $(\tau)$ was calculated using $P(t)=P(0)$ - $\exp (-t / \tau)$, where $P(t)=L V$ pressure at time $=t$ and $P(0) L V$ pressure at time $\mathrm{LVdP} / \mathrm{dtmin}$.

\section{Determination of regional ventricular geometry}

For each measurement, 3 consecutive end-diastolic video images were digitized off-line by use of a video frame grabber $(8$ bits gray-scale, 768 × 578 pix, DT3155, Data Translation, Inc., Marlboro, MA). The digitized images were analyzed by use of NIH Image software (V 1.52) by an experienced echocardiographist who was unaware of the specific time points of the images. Regional geometry (wall thickness and wall volume; see below) was determined within 6 wall sectors, as depicted in Figure 5.1. Sectors 1 through 3 and 6 are situated at the LV free wall, sectors 4 and 5 at the interventricular septum. In the echocardiographical images, sector 6 was not always clearly visible and therefore was excluded from the analysis. In all animals, the location of the pacing lead fell withir wall sector 2 , and sector 5 was most remote from this sector.

In the analysis of the 2D-echo images, a total of 50-70 contour points on the endocardial, epicardial and papillary contours were marked manually (Figure 5.1A and B). Epi and endocardial contour coordinates were converted to a polar representation, with the center of the LV cavity as the origin and the bisector of the angle between the papillary muscles and the center of the LV cavity as $0^{\circ}$ reference. Inner $\left(r_{i}\right)$ and outer $\left(r_{0}\right)$ radin in the sectors were determined by fitting the original epicardial and endocardial contourpoints to a model to limit the highest circular frequency to the fourth harmonic:

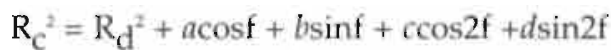

$$
\begin{aligned}
& +e \cos 3 \mathrm{f}+f \sin 3 \mathrm{f}+g \cos 4 \mathrm{f}+h \sin 4 \mathrm{f}
\end{aligned}
$$

where $R_{c}$ and $R_{d}$ are the calculated and measured radius $\left(r_{i}\right.$ or $\left.r_{0}\right)$, respectively, and $a$ through $h$ are constants. Wall thickness (WT) of a sector was calculated as $\mathrm{WT}=\mathrm{r}_{\mathrm{o}}-\mathrm{r}_{\mathrm{i}}$. Wall sector area $\left(\mathrm{A}_{\text {sector }}\right)$ was derived from the thus obtained $r_{j}$ or $r_{0}$ by integration over each sector (Figure 5.1D). Sector wall volume ( $\mathrm{V}_{\text {sector }}$ ) was calculated as $A_{\text {sector }} \cdot{ }^{-I_{m}}$, assuming that growth in the radial and base to apex direction was equal. The median radius $\left(r_{m}\right)=\left[\left(r_{0}{ }^{2}+r_{i}{ }^{2}\right) / 2\right]^{0.5}$. 

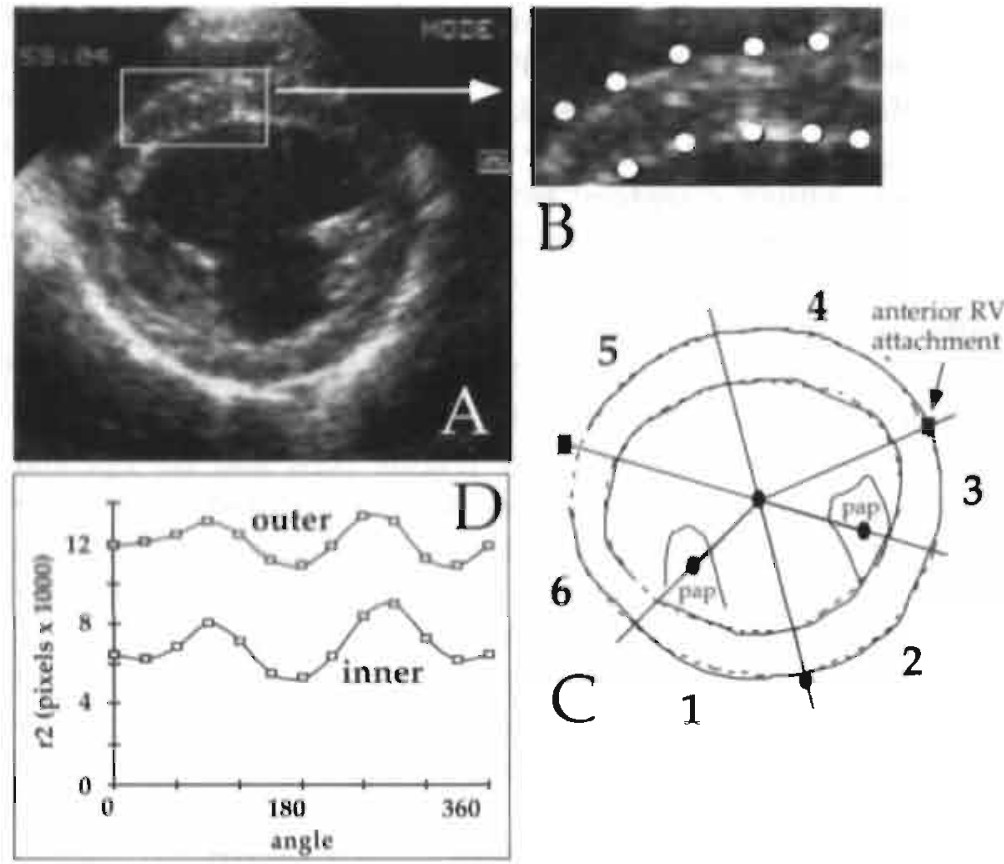

Intraobserver and interobserver variability for the measurements of regional wall thickness were $5.7 \%$ and $5.8 \%$, respectively.

\section{Determinations of global LV dimensions}

Cavity and wall volume of the entire LV were calculated from the cross-sectional images and long axis dimensions by use of cylinder-ellipsoid model calculations. ${ }^{1020}$ We correlated LV wall volume at $\mathrm{t}=6$ months with the gravimetrically determined postmortem LV mass.
Figure 5.1 Schematic representation of method to analyze echocardiographic images. In digitized echocardiographic cross sections of the left ventricle (A) the inner and outer contours are marked at 50-70 sites (B). Contours ane calculated from the set of endocardial and epicandial contour points using modified Fourier analysis (Equation 1). C, Contours draton by connecting the marked points (broken lines) and calculated contours (drawn lines). Also indicated are the 6 sectors, which are defined by the following anatomical landmarks: centers of anterior and posterior pupillary muscles and center of anterior attachment of right sentricular (RV) unall to left ventricular (LV) wall. $D$, Square value of the outer and inner nadii $\left(r^{2}\right.$, in pixels $)$ as a function of the angle, $0^{*}$ being the line separating sector 1 and 2 , as depicted in $C$.

\section{Statistical analysis}

Paired hemodynamic data were analyzed using a Wilcoxon signed rank test, the Mann-Whitney- $U$ test was used to evaluate differences between groups. For morphometric data, the samples were first assessed for normality of distribution by the Kolmogorov-Smirnow test. Then, a nested analysis of variance (ANOVA) was used.15 ANOVA for repeated measurements was used to evaluate changes of echocardiographic variables during the course of the experiment. If significant differences were found, significant points were isolated using Bonferroni-Dunn correction. Data are presented as mean $\pm 1 \mathrm{SD}$. $\mathrm{P}<0.05$ was considered significant.

\section{RESULTS}

No dog in this study showed signs of cardiac failure or other illnesses during the entire study period. In all dogs in the PACE group, cardiac pacing was possible throughout the study period. 


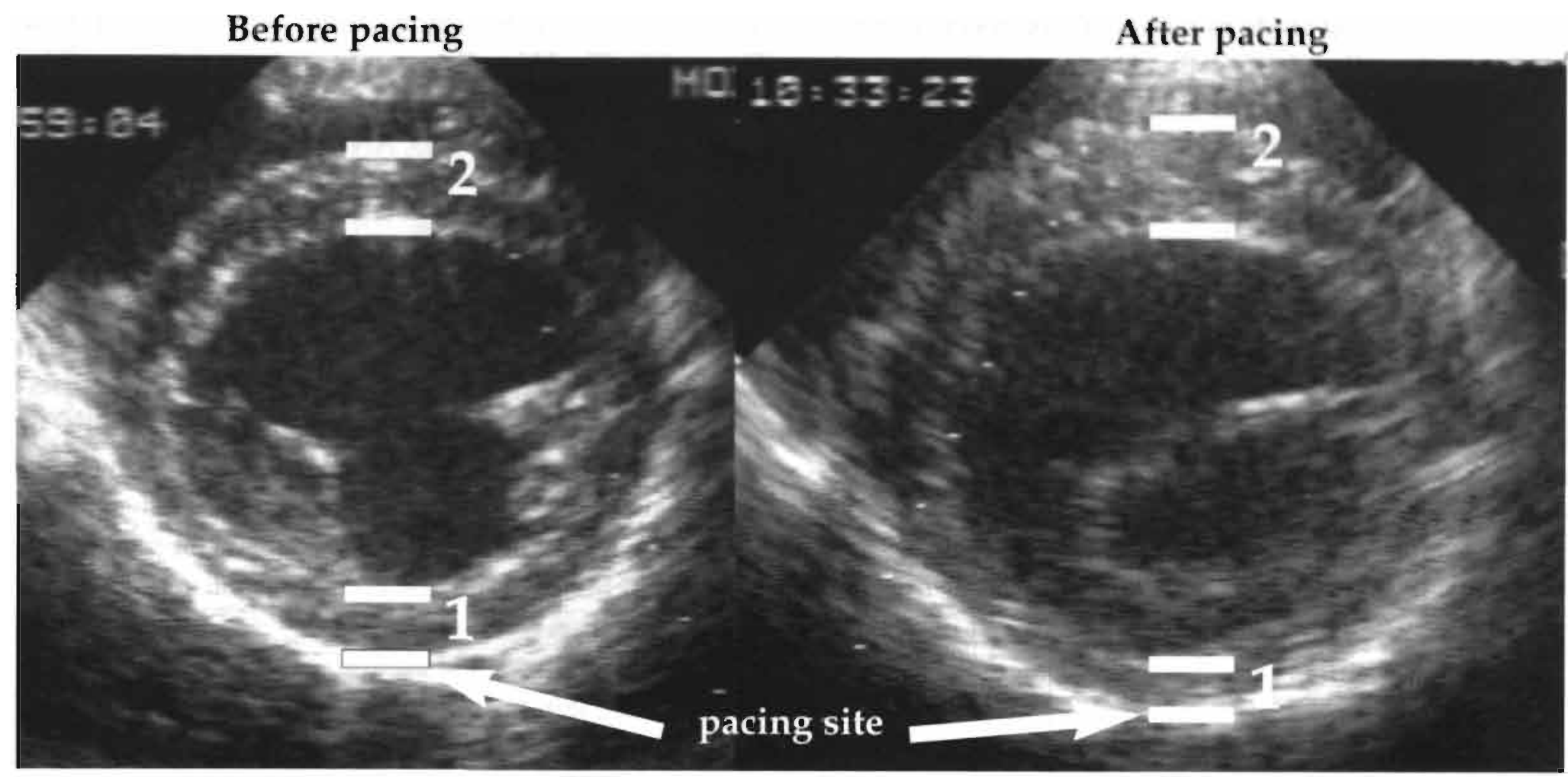

Figure 5.2 Cross sectional LV short axis echocardiographic images, recorded from a dog before and six months after onset of LV free wall pacing. Note the decreased LV free wall thickness (1), the increased septal wall thickness (2) and the increased LV cavity in the image after 6 months of pacing compared with baseline image.

\section{Echocardiographic changes}

Figure 5.2 shows representative echocardiographic images of a heart before and 6 months after onset of pacing at the LV free wall. These images illustrate that 
ventricular pacing leads to global enlargement of the LV cavity and wall, whereas the LV free wall (the earlyactivated region) becomes thinner and the septum (the late-activated region) becomes thicker.

\section{Global changes}

In the SHAM group $(n=5), L V$ cavity volume and wall mass remained constant during the experimental period (data not shown). In the PACE group $(n=8), L V$ cavity

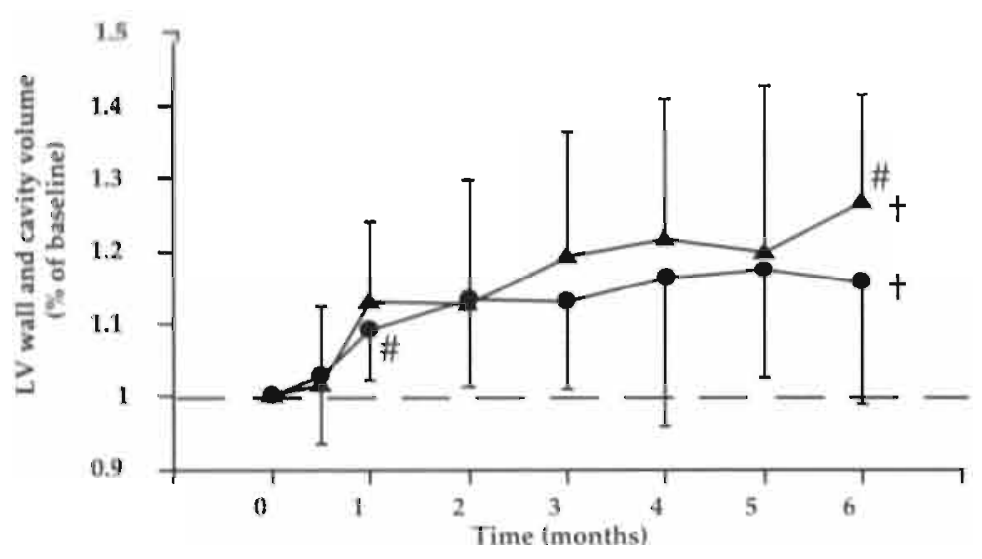

Figure 5.3 Changes in global LV cavity volume and wall mass during 6 months of ventricular pacing. + significant change over time by ANOVA; \# $p<0.05$ compared with baseline. volume and LV mass significantly increased over time (Figure 5.3). The LV vall-to-cavity area ratio, decreased by $7 \pm 11 \%$ and $10 \pm 16 \%$ after 1 and 6 months of ventricular pacing, respectively $(p<0.05$, ANOVA).

\section{Regional changes}

Within 1 morth of ventricular pacing wall thickness tended to decrease in the early-activated LV free wall and to increase in the late-activated septum (Figure 5.4A): the LV free wall/septum thickness ratio decreased significantly by $17 \pm 12 \%$. Between 1 and 6 months of pacing, this ratio further decreased to $33 \pm 15 \%$ below baseline, owing to a $23 \pm 12 \%$ increase in septal thickness and a $17 \pm 17 \%$ decrease in LV free wall thickness compared with baseline. In SHAM animals, no changes in regional geometry were observed (Figure 5.4A).

Compared with baseline, sector volume of the septum was significantly increased by $20 \pm 16 \%$ after 1 month and by $39 \pm 13 \%$ after 6 months of pacing, but sector volume of the LV free wall did not change significantly (Figure 5.4B). Sector volume did not significantly change in regions 1 and 3 (adjacent to the earliest activated $L V$ free wall region; $-0.7 \pm 10.4 \%$ and $11.1 \pm 15.4 \%$, respectively; (see Figure 5.1) but significantly increased in sector 4 , adjacent 

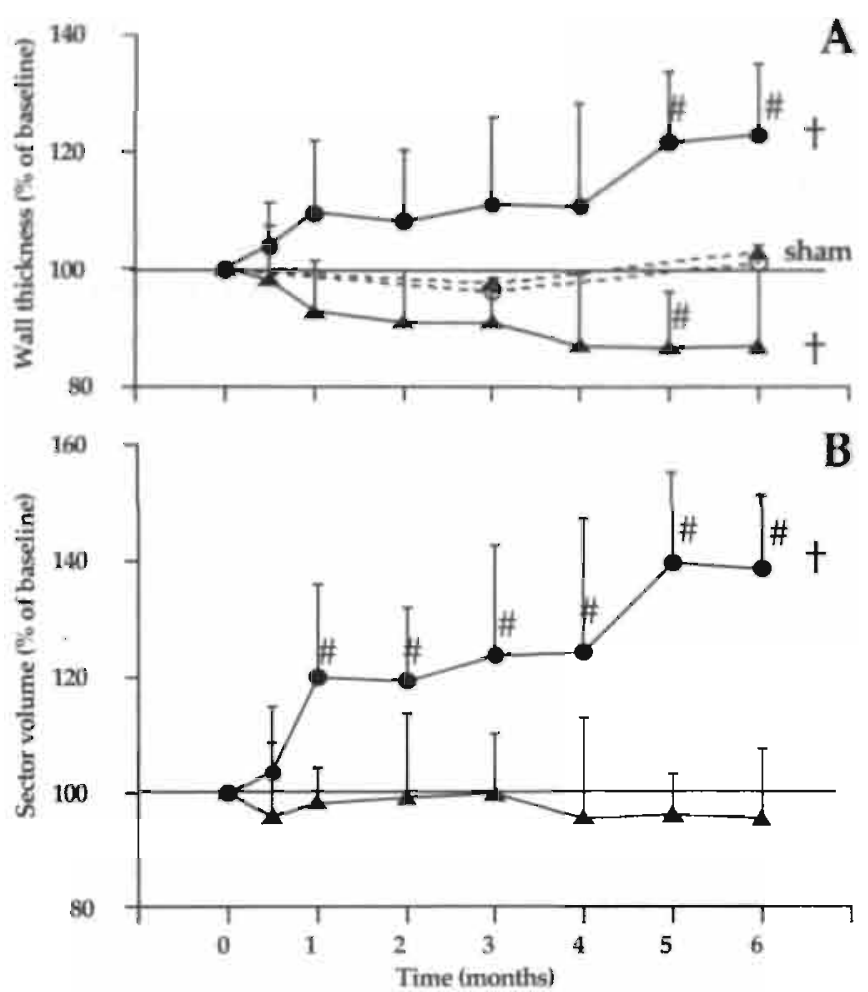

Figure5.4: Changes in wall thickness (top) and wall sector volume (bottom) over time in the early-activated LV free wall ( $\mathbf{\Delta}$, sector 2 in Figure 5.1) and the late-actioated septum (- sector 5 in Figure 5.1). Open symbols are corresponding regions in the sham group. + significant change over time by ANOVA; \# $p<0.05$ compared with baseline. to the most remote septal region $(30.3 \pm 15.3 \%)$.

\section{Post mortem observations}

The echocardiographically determined LV wall volume ( $\left(V_{\text {echo }}\right)$ was highly correlated with post mortem $\mathrm{LV}$ weight ( $\left.\mathrm{LV}_{\text {post mortem }}\right)$ and the relation could be described by a linear relation:

$$
\text { (2) } \mathrm{LV}_{\text {post mortem }}=-1.78+1.23 \cdot \mathrm{LV}_{\text {echo }}, \mathrm{r}=0.92
$$

The LV/body weight ratio was significantly larger in PACE than in SHAM animals $(6.16 \pm 0.85$ and $4.91 \pm 0.47 \mathrm{~g} / \mathrm{kg}$, respectively).

In the PACE group, myocytes were significantly thicker in the septum $(24.2 \pm 2.6 \mathrm{~mm})$ than in the LV free wall $(20.6 \pm 1.7 \mathrm{~mm})$. In the SHAM group myocyte thickness was not significantly different in these regions $(22.3 \pm 1.9$ and $22.3 \pm 3.0 \mathrm{~mm}$, respectively, Figure 5.5$)$. The free wall-to-septum ratio of myocyte diameter was significantly smaller in the PACE than in the SHAM group $(0.82 \pm 0.07$ and $0.99 \pm 0.09$, respectively).

Ventricular pacing did not influence the myocardial collagen fraction. The collagen fraction in the LV free wall and septum was $4.1 \pm 0.7 \%$ and $4.6 \pm 0.3 \%$, respecti- 


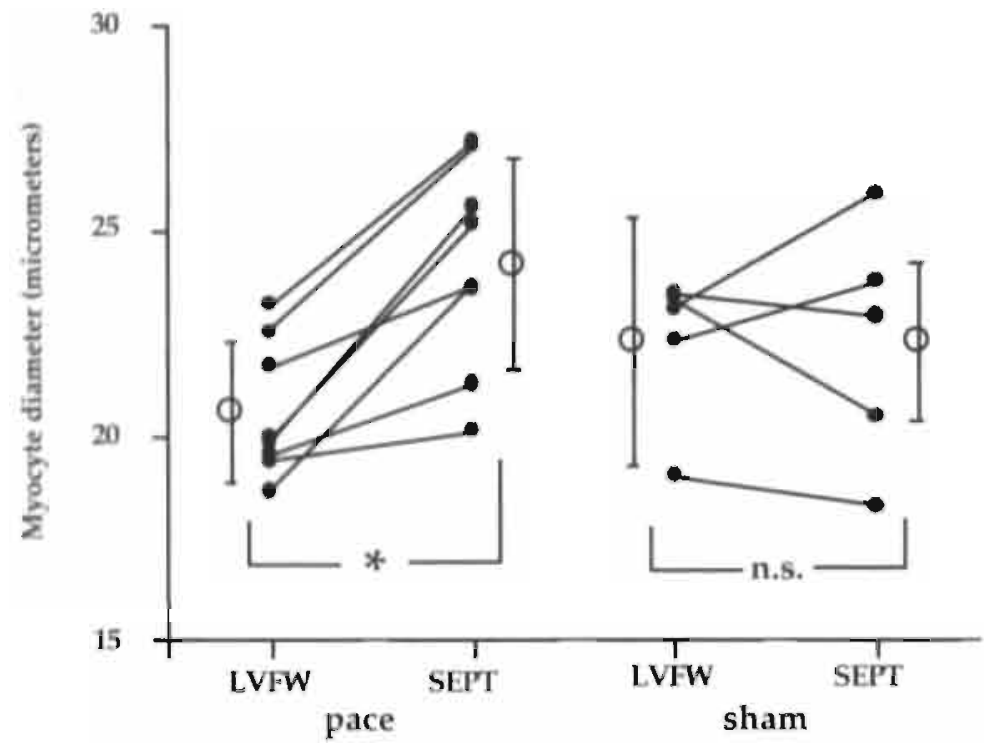

Figure 5.5 Myocyte diameter in the LV free wall (LVFW) and septum (SEPT) of the PACE and SHAM groups. Each closed symbol is the mean value of 100 myocytes from one individual region. All groups of myocytes were distributed normally. Lines connect data from the same animal. SD of the diameter of 100 myocytes ranged from 3.8 to $7.2 \mu \mathrm{m}$. Open symbols and bars indicate mean values and SD per negion. " $p<0.05$ between LV free wall and septum of the same heart (nested analysis ANOVA). n.s.: not significantly different. vely, in the PACE group, and $4.0 \pm 0.8 \%$ and $4.1 \pm 0.7 \%$, respectively, in the SHAM group.

\section{Electrophysiology and hemodynamics}

After 15 min of ventricular pacing the duration of the QRS complex more than doubled as compared with sinus rhythm (Table 5.1). After 6 months of pacing the width of the QRS complex further increased significantly by $20 \pm 23 \%$ of the value after $15 \mathrm{~min}$ of pacing.

During the implantation procedure, hemodynamics were not significantly different between the SHAM and PACE groups, and the hemodynamic effects of pacing were similar in both groups (Table 5.1). Pacing significantly reduced stroke volume index, dPlv/dtmax and $\mathrm{dPlv} / \mathrm{dtmin}$ and significantly increased heart rate. Pacing increased end-diastolic LV pressure significantly in the PACE group, but the increase did not reach the level of significance in the SHAM group. Systolic LV pressure and cardiac index did not change significantly compared with sinus rhythm (Table 5.1). Pressure-volume analysis showed that ventricular pacing significantly increased the slope of the end-systolic pressure-volume relationship but also the volume at which end-systolic LV pressure reached a value of $75 \mathrm{mmHg}$ (V75, see Figure 5.6 for examples). 


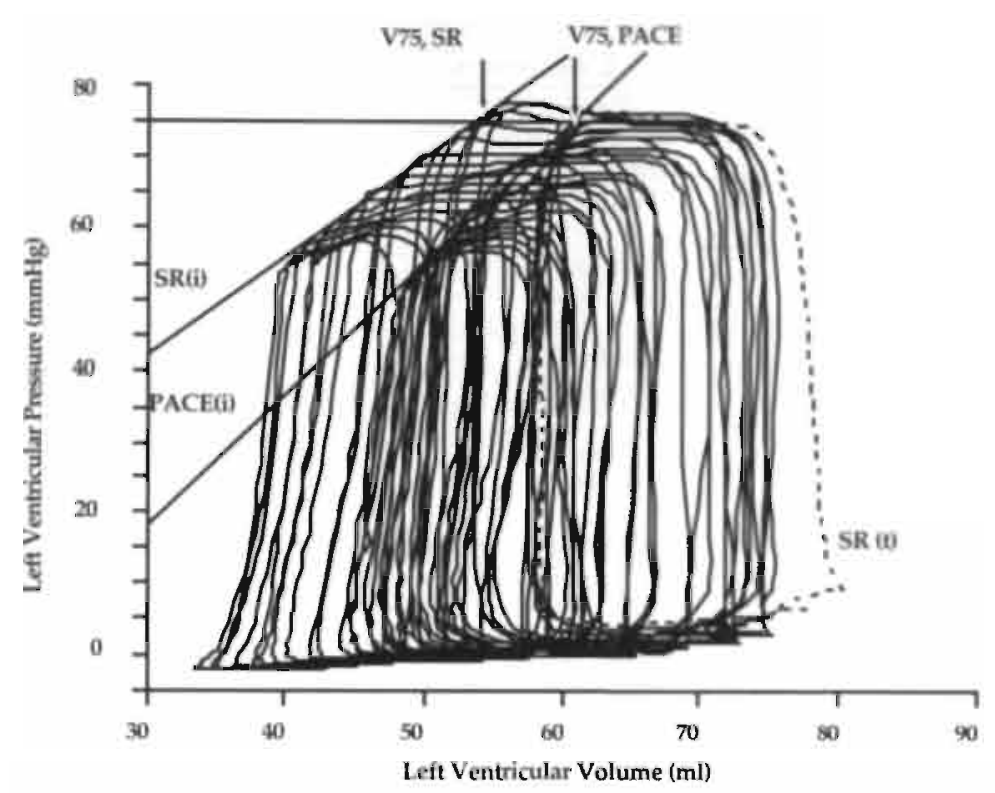

Figure 5.6. Pressure-volume diagrams during simus rhythm (SR) and ventricular pacing at implantation (index $i$ ). Thickly drawn lines represent a steady-state heartbeat. Thinner lines represent loops during progressive preload reduction. Regression lines connecting the end-systolic points (endsystolic pressure-volume relation) during SR and pacing are also indicated. Note the rightward shift of end-systolic pressure-volume relation during ventricular pacing. Volume at which an end-systolic pressure of $75 \mathrm{mmHg}$ is reached (V75) is indicated by arrows. Broken line indicates steady-state pressure-volume relation during SR after 6 months of pacing. Note the rightwand shift of the latter loop as compared with loop during $S R$ at implantation.
Ventricular pacing did not acutely change end-diastolic LV volume (Table 5.1).

After 6 months of pacing, hemodynamic variables except for heart rate and end-diastolic LV pressure, during sinus rhythm and ventricular pacing were not significantly different during the terminal procedure from the corresponding values during implantation (Table 5.1). In both groups, the hemodynamic changes due to the switch from ventricular pacing to sinus rhythm were not significantly different in the implantation and the termination procedure.

\section{DISCUSSION}

The findings in the present study demonstrate that long-term asynchronous electrical activation, as induced by ventricular pacing, leads to increased LV cavity volume and wall mass and asymmetrical changes in LV wall thickness. The early-activated regions become thinner and the late-activated regions become thicker. This asymmetry in wall thickness is associated with unchanged sector wall volume in early-activated regions and increased sector wall volume in late-activated regions. The increase in sector wall volume in the late activated regions results 


\section{Chapter 5}

\begin{tabular}{|c|c|c|c|c|c|c|c|c|}
\hline \multirow[b]{2}{*}{ Variable Group } & \multicolumn{3}{|c|}{ Implantation } & \multicolumn{5}{|c|}{ Termination } \\
\hline & SR & Pace & $\mathrm{S} / \mathrm{P}$ & SR & Pace & $\mathrm{S} / \mathrm{P}$ & $S / S$ & $\mathrm{P} / \mathrm{P}$ \\
\hline \multicolumn{9}{|c|}{ QRS duration (ms) } \\
\hline pace & $51.9 \pm 10.6$ & $112.6 \pm 18.5$ & $\dot{*}$ & $59.4 \pm 7.8$ & $13 ! .8+25.2$ & • & & • \\
\hline sham & $39.8 \pm 5.0$ & $115.6 \pm 14.6$ & $\bullet$ & $40.6 \pm 4.6$ & $1112+13.1$ & & & \\
\hline \multicolumn{9}{|l|}{ HR (bpm) } \\
\hline pace & $104.6 \pm 18.5$ & $110.2 \pm 19.7$ & * & $110.2 \pm 19.7$ & $126,4 \pm 24.2$ & $\cdot$ & & * \\
\hline \multirow{2}{*}{\multicolumn{2}{|c|}{ PLVsys (mmHg) }} & $127.8 \pm 20.8$ & wentuse & $107.2 \pm 12.6$ & $12.1 \pm 8.4$ & & & \\
\hline pace & $86.8 \pm 11.2$ & $87.3 \pm 11.5$ & & $93.4 \pm 13.2$ & $91.8 \pm 11.9$ & & & \\
\hline sham & $91.2 \pm 13.3$ & $84.9 \pm 5.3$ & 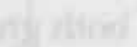 & $101 \pm 14.17$ & $98.7 \pm 10.7$ & & & \\
\hline \multicolumn{9}{|l|}{ PLVed $(\mathrm{mmH})$} \\
\hline pace & $8.2+2.7$ & $10.1 \pm 3.2$ & 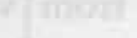 & $6.9 \pm 4.6$ & $5.8 \pm 4.4$ & * & & * \\
\hline $\begin{array}{l}\text { sham } \\
\mathrm{SVI}(\mathrm{ml} / \mathrm{m} 2)\end{array}$ & $7.1 \pm 2.3$ & $7.9 \pm 1.6$ & & $8.9 \pm 2.8$ & $8.0 \pm 1.2$ & & & \\
\hline \multicolumn{7}{|l|}{$\mathrm{SVI}(\mathrm{ml} / \mathrm{m} 2)$} & & \\
\hline $\begin{array}{l}\text { pace } \\
\text { sham }\end{array}$ & $22.7 \pm 3.4$ & $18.1 \pm 5.1$ & & $\begin{array}{l}30.2 \pm 3.0 \\
29.2 \pm 6.6\end{array}$ & $24.3 \pm 5.6$ & 4 & * & \\
\hline \multicolumn{9}{|c|}{$\mathrm{dP} / \mathrm{d} \mathrm{t} \max (\mathrm{mmH} / \mathrm{g} / \mathrm{s})$} \\
\hline pace & $1125 \pm 228$ & $1054+202$ & & $1359 \pm 389$ & $1238 \pm 335$ & & & \\
\hline sham & $1283 \pm 174$ & $1176 \pm 120$ & & $1336 \pm 167$ & $1253 \pm 178$ & & & \\
\hline \multicolumn{9}{|c|}{$\mathrm{dP} / \mathrm{dt} \min (\mathrm{mmHg} / \mathrm{s})$} \\
\hline pace & $1128+212$ & $968 \pm 120$ & & $1336 \pm 358$ & $1216 \pm 343$ & & 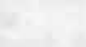 & \\
\hline sham & $1366 \pm 327$ & $1102+302$ & & $1432+172$ & $1270 \pm 159$ & & & \\
\hline \multicolumn{9}{|l|}{ Tiuu (ms) } \\
\hline $\begin{array}{l}\text { pace } \\
\text { sham }\end{array}$ & $\begin{array}{l}38.1 \pm 5.4 \\
37.3+8.6\end{array}$ & $7.6 \pm 9.1$ & * & $36.0 \pm 9.7$ & $37.2 \pm 11.3$ & & & \\
\hline \multicolumn{9}{|l|}{ Ees $(\mathrm{mmH} / \mathrm{g} / \mathrm{mi})$} \\
\hline pace & $1.18 \pm 0.70$ & $1.38 \pm 0.58$ & $1 / 4$ & $0.90 \pm 0.22$ & $1.12 \pm 0.16$ & $0 / 4$ & $1 / 4$ & $1 / 3$ \\
\hline sham & $1.53 \pm 0.76$ & $2.77 \pm 1.67$ & $1 / 4$ & $1.20 \pm 0.39$ & $1.91 \pm 0.79$ & $1 / 4$ & $1 / 4$ & $1 / 4$ \\
\hline \multicolumn{9}{|l|}{$\mathrm{V} 75(\mathrm{mi})$} \\
\hline pace & $42.1 \pm 17.1$ & $53.1 \pm 20.3$ & $1 / 4$ & $42.3 \pm 14.4$ & $47.0 \pm 19.8$ & $2 / 2$ & $2 / 2$ & $1 / 3$ \\
\hline \multicolumn{7}{|l|}{$\operatorname{EDV}(\mathrm{ml})$} & $1 / 4$ & $1 / 4$ \\
\hline pace & $78.3 \pm 21.8$ & $79.5 \pm 23.6$ & $2 / 3$ & $85.5 \pm 30.1$ & $84.1 \pm 21.1$ & $2 / 2$ & $4 / 0$ & $2 / 2$ \\
\hline sham & $54.1 \pm 13.3$ & $52.2 \pm 13.6$ & $2 / 3$ & $67.1 \pm 26.5$ & $63.9 \pm 21.8$ & $3 / 2$ & $5 / 0$ & $4 / 1$ \\
\hline \multicolumn{8}{|l|}{$\mathrm{EF}(\%)$} & \\
\hline $\begin{array}{l}\text { Pace } \\
\text { sham }\end{array}$ & $38.6 \pm 5.7$ & $39.1 \pm 9.0$ & $2 / 3$ & $43.1 \pm 9.7$ & $39.1 \pm 8.8$ & $4 / 1$ & $4 / 1$ & $2 / 3$ \\
\hline
\end{tabular}


Table 5.1 Hemodynamic effects of ventricular pucing during the implantation procadure and 6 months later, during the termantion procedure

$=p<0.05$ for following comparisons:

S/P: ventricular pacing as compared with sinus rhythen at the same day

S/S: values of simus rhythm during implanticion as compared with those during termination

P/P: values of ventricular pacing during implantation as conmand with those during termination

Data on LV pressure-rolume measuranents were obtainat in 5 animals of both groups, except for termination in the PACE group ( $n=4)$

\#/\# : number of cases where oalues were higher/lower during termination than during implantation

from growth of cardiomyocytes and a proportional increase in collagen content. These data indicate that longterm asynchronous electrical activation induces asymmetrical hypertrophy and ventricular enlargement. Because workload has been shown to be lower in early than in late activated regions, ${ }^{23}$ the findings also indicate that local cardiac load is an important regulator of local cardiac growth.

\section{Asymmetrical hypertrophy}

Asymmetry in hypertrophy is most likely related to the pronounced regional differences in contraction pattern during ventricular pacing. In early-activated regions, rapid early systolic shortening is followed by strongly reduced shortening later in systole. In contrast, in late-activated regions, considerable early systolic prestretch is followed by pronounced systolic shortening. ${ }^{242}$ Although stretch has been applied frequently to evoke growth responses in isolated myocytes"' the real stimulus for hypertrophy is as yet unknown. In mathematical model studies Arts et al." simulated structural adaptation of the LV wall to pressure and volume overload. They proposed that the development of hypertrophy of the entire left ventricle can be explained by local myocyte growth that is regulated by early systolic stretch. On the basis of the local stretch patterns mentioned above, selective hypertrophy and wall thickening in lateactivated septum and the absence of hypertrophy in the early-activated LV free wall are in accordance with the theory of Arts et al.9 More in general, the present data comply with the idea that local cardiac load is an important determinant of local cardiac growth, as

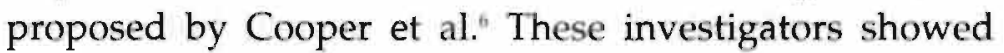
papillary muscle atrophy after cutting its chordae tendinae. In the experiments of Cooper et al. ${ }^{6}$ and in the present study, all myocardial regions were subjected to the same plasma levels of potentially growth-promoting humoral factors like noradrenaline and angiotensin II. ${ }^{4,5}$ This is important because in many experimental and 
pathological conditions potential growth promoting actions of noradrenaline or angiotensin may have been confounded by their hemodynamic effects (see reference 22 for review). The results of the present study do not exclude a role of autocrine or paracrine angiotensin release in mediating stretch-induced myocyte growth. ${ }^{x \geq 3}$

Locally different growth is demonstrated both echocardiographically and histologically. The relative changes in myocyte diameter, however, appear to be less pronounced than those in wall thickness. Therefore, other factors such as increased myocyte length and hyperplasia may have contributed to macroscopic growth. Although hyperplasia is usually confined to more severe degrees of hypertrophy, its presence can not be excluded in the present study.

The early-activated $L V$ free wall probably becomes thinner owing to LV cavity dilation. This dilation did not occur immediately after onset of pacing, but became evident after one month of pacing. The cavity dilation may be a secondary stimulus for hypertrophy throughout the LV wall, which may have enforced growth in the late activated regions and may have prevented atrophy to occur in the early activated regions.

The histological measurements indicate that after 6 months of pacing, colagen fractions have not changed. This implies that locally the collagen content increased in proportion with myocyte growth. Hypertrophy with unchanged collagen factions is also seen with volume overload hypertrophy ${ }^{25}$ In other forms of hypertrophy, however, collagen fractions are increased, presumably owing to high plasma levels of angiotensin or aldosterone.*

\section{LV pump function}

As shown by others ${ }^{1,220-12,27}$ ventricular pacing acutely reduces global ventricular function. The present study demonstrates that this reduction is similar after 15 minutes and 6 months of pacing. The observation that ventricular function recovers as much when pacing is stopped after 6 months as it decreases when pacing is started during implantation indicates that the myocardium is not failing and that the asymmetric hypertrophy is still compensated.

Pacing with $30 \mathrm{~ms}$ AV interval did not change enddiastolic volume and tended to icnrease end-diastolic LV pressure (Table 5.1), indicating that ventricular preload was not reduced and that the observed decrease in contractility is due to the asynchronous electrical activation. Ventricular pacing did reduce early ventricular 
relaxation, as indicated by the decrease in $\mathrm{LVdP} / \mathrm{dtmin}$ and the increased $\tau$.

In the present study, we used AV sequential pacing with a short $\mathrm{AV}$ interval $(30 \mathrm{~ms})$ to ensure activation of the entire ventricle from the ectopic site. This approach was preferred above induction of AV block, which may cause myocardial damage, potentially interfering with the structural adaptations to be studied. Moreover, the setup used enabled us to study LV function during ventricular pacing and sinus rhythm at termination of the experiment as well. In patients, changing the AV interval from 100-130 to $0 \mathrm{~ms}$ decreased cardiac output by approximately $20 \%{ }^{122829}$ In a separate series of experiments in $5 \mathrm{AV}$ blocked dogs, however, we found that switching the AV interval from $100 \mathrm{~ms}$ to $25-30 \mathrm{~ms}$ changed cardiac output only by -10 to $+3 \%$ (Peschar and Prinzen, unpublished data, 1997). This is in agreement with the observation of Rosenqvist et al., ${ }^{30}$ that hemodynamic performance in dogs is not influenced when the AV interval is varied between 60 and $150 \mathrm{~ms}$. Therefore, in dogs pacing at short AV interval seems to affect cardiac pump function only to a minor degree. Most importantly, even if the short AV interval had impaired global ventricular function, it is very unlikely that it would have caused the asymmetric hypertrophy as induced by asynchronous electrical activation, the major finding in the present study.

\section{Pacing site}

The site of ventricular pacing in the present study (base of the LV free wall) is different from the one used clinically, that is, the RV apex. The LV free wall was chosen as site of pacing because in this situation, myocardial wall thickness in the early activated LV free wall and in the late-activated septum could be determined in one shortaxis echocardiographic image. Despite this difference, the findings in the present study are clinically relevant because comparable degrees of asynchrony are obtained during pacing at the RV apex and at the LV free wall.' Moreover, in the present study the duration of the QRS complex during pacing was similar to that during RV apex pacing in both dogs ${ }^{30}$ and humans. In addition, in a recent study, using MRI tagging, we were able to show that both LV base and RV apex pacing create a more than doubling of the heterogeneity of regional workload as compared to atrial pacing. Of course, the sites of early and late activation were at virtually opposite locations during the two modes of pacing. ${ }^{21}$

Our findings are the first to show that in vivo 
asynchronous electrical activation can lead to locally different growth responses within the same ventricle of adult hearts of a large animal species that are presumably quite comparable to human hearts. It would be of interest to know whether pacing at a site causing less asynchrony, such as the high ventricular septum, ${ }^{x}$ leads to a lesser degree of asymmetrical hypertrophy, especially because pacing from this site resulted in fewer histological abnormalities than pacing from the RV apex.".

\section{Conclusions}

The present study shows that chronic asynchronous activation of the ventricles leads to asymmetrical hypertrophy. This demonstrates that in the LV local cardiac mass is a function of local cardiac load, which is higher in late- than in early-activated regions, and emphasizes the importance of physiological, fairly synchronous electrical activation of the left ventricle.

\section{REFERENCES}

1. Lister JW, Klotz DH. Jomain SL_. Stuckey JH, Hoffman BF. Effect of pacemaker site on cardiac output and ventricular activation in dogs with complete heart block. Am ] Cardiol 1964: 14:494-503.

2. Prinzen FW, Augustijn CH, Arts T, Allessie MA, Reneman RS, Redistribution of myocardial fiber strain and blood flow by asynchronous activation. Am I Physiol $1990 ; 259: \mathrm{H} 300-\mathrm{H} 308$
3. Delhaas T, Arts T, Prinzen FW, Reneman RS. Regional fibre stress-fibre strain area as estimate of regional blood flow and oxygen demand in the canine heart. I Physiol (London) 1994: 47734481-496.

4. Dzau VJ. Tissue renin-angiotensin system in myocardial hypertrophy and failure. Arch Int Med 1993; 1539937-942.

5. Long CS, Kariya K, Karns L. Simpson PC. Trophic factors for cardiac myocytes. J Hypertension 1990; 8 (suppt. 7) $\$ 219-\$ 223$.

6. Cooper IV G, Kent RL, Uboh CE, Thompson EW, Marino TA. Hemodynamic versus adrenergic control of cat right ventricular hypertrophy. I Clin Invest 1985; 75:1403-1414.

7. Sadoshima II, Takahashi T, Jahn. L. Irumo S Roles of mechano-sensitive ion channels, cytoskeleton, and contractile activiry in stretch-induced immediategene expression and hypertrophy of cardiac myocytes. Proc Nat Acad Sci USA. Cell Biol 1992; 89-9905-9909.

8. Kent RL. McDermott PJ. Passive load and angiotensin II evoke differential nesponses of gene expression and protein synthesis in cardiac myocytes. Circ Res $1996 ; 78: 829-838$.

9. Arts T, Prinzen FW, Snoeckx LHEH, Rijcken JM, Reneman RS. Adaptation of cardiac structure by mechanical teedback in the environment of the cell: A model study. Biophysical J 1994; 66:953-961.

10. Park RC little. WC, O'Rourke RA. Effect of alteration of left ventricular activation sequence on the left ventricular end-systolic pressure-volume relation in closed-chest dogs. Circ Res 1985; 57:706-71\%.

11. Burkhoff D, Oikawa RY, Sagawa K. Influence of pacing site on left ventricular contraction. Am J Physiol 1986; 251:H428-H435.

12. Daggett WM, Bianco JA. Powell WI. Austen WG. Relative contribution of the atrial systole-ventricular systole interval and of patterns of ventricular activation to ventricular function during electrical pacing of the dog heart. Circ Res 1970; 27:69-79.

13. Baan J, Van Der Velde ET, De Bruin HG, Smeenk G, Koops J. Van Dijk A, Temmerman D, Senden J, Buis B. Continuous measurement of left ventricular volume in animals and hurnans by conductance catheter. Circulation 1984: 70:812-823.

14. Vliegen HW, Laarse van der A, Huysman JAN, Wijnvoord EC, Mentar M, Cornelisse Cl, Eulderink F. Morphometric quantification of myocyte dimensions validated in normal growing rat hearts and applied to hypertrophic human hearts. Cardiovasc. Res 1987; 21:352-357.

15. Urabe Y, Mann DL., Kent Ri., Nakano K, Tomanek RJ, Carabello BA, Cooper IV G. Cellular and ventricular contractile dysfunction in experimental canine mitral regurgitation. Circ Res 1992; 70:131-147

16. Junqueira LCU, Bignolas. G. Brentani RR. Picrosirius staining plus polarization microscopy, a specific method for collagen detection in tissue sections. Histochem ] 1979; 11:447-455: 
17. Van Krimpen C, Smits JFM, Cleutjens JPM, Debets IMM. Schoemaker RG, Struyker-Boudier HA, Bosman FT, Daemen MIAP. DNA synthesis in the noninfarcted cardiac interstitium after left coronary artery ligation in the rat effects of captopril. I Mol Cell Cardiol 1991; 23:1245-1253.

18. Marino P, Kass D, Lima J, Maughan WL, Graves W, Weiss IL Influence of vite of regional ischemia on LV cavity shape change in dogs Am I Physiol 1988; 254:H547-H557.

19. Gaynor JW, Feneley MI, Gall SA, Maier GW, Kisslo IA, Davis IW, Rankin IS, Glower DD. Measurements of left ventricular volume on normal and volumeoverloaded canine hearts. Am J Physiol 1994; 2f,6: $1329-\mathrm{H}_{3} 40$.

20. Wyatt HI. Heng MK, Meerbaum S, Hestenes ID, Cobo JM, Davidson RM, Corday E. Cross-selectional echocardiography. I. Analysis of mathematic models for quantifying mass of the left ventricle in doga. Circulation 1979; 60-1104-1113

21. Prinzen FW, Hunter WC. McVeigh ER. Mapping of contractile inhomogeneity

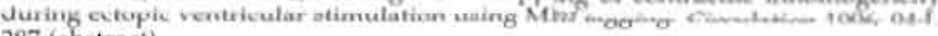
387 (abstract).

22. Cooper IV G. Cardiocyte adaptation to chronically altered load. Ann Rev Physiol 1987: 49:501-518.

23. Sadoshima J, Xu Y, Slayter HS, lzumo S. Autocrine release of angiotensin II mediates stretch-induced hypertrophy of cardiac myocytes in vitro. Cell 1993; 75.977-984

24. Anversa P, Kajstura J, Cheng W, Reiss K, Cigola E, Olivetri G. Insulin-like growth factor-1 and myocyte growth: the danger of a dogma. Part II. Induced myocardial growth: pathologic hypertrophy. Cardiovase Res 1996; 32:484-495

25. Michel J-B, Salzmann J-L, Nlom MO, Bruneval P, Barres D, Camilleri J-P. Morphometric analysis of collagen network and plasma perfused capillary bed in the myocardium of rats during evolution of cardiac hypertrophy. Basic Res Cardiol 1986; 81:142-154

26. Brilla CG, Pick R, Tan LP, Janicki IS. Weber KT. Remodeling of the rat right and left ventricles in experimental hypertension. Circ Res 1990; 67:1355-1364.

27. Rosenqvist M, Isaaz K, Botvinick EH, Dae MW, Cockrell J, Abbott JA, Schiller NB, Griffin IC. Relative importance of activation sequence compared to atrioventricular synchrony in left ventricular function. Am J Cardiol 1991; 67:148-156.

28. Samet P, Castillo P, Bemstein WH. Hemodynamic consequences of sequential atrioventricular pacing. Am J Cardiol 1968;21:207-212.

29. Faerestrand S, Oie B, Ohm O-f. Noninvasive assessment by doppler and M-mode echocardiography of hemodynamic responses to temporary pacing and to ventriculoatrial conduction. PACE 1987; 10:871-885.

30. Rosenqvist M, Bergfeldt L, Haga Y, Ryden I, Ryden L Owali A. The effect of ventricular activation sequence on cardiac performance during pacing. PACE 1996; 19:1279-1286

31. Vassallo JA, Cassidy DM, Miller IM, Buxton AE, Marchlinski FE, Josephson ME. Left ventricular endocardial activation during right ventricular pacing: effect of underlying heart disease. I Am Coll Candiol 1986; 7:1228-1233.
32. Karpawich PP, Justice $C D$, Chang C.H. Gause CY, Kuhns L.R. Septal ventriculat pacing in the immature canine heart A new perspective. Am Heart I 1991; 21-827-833. 


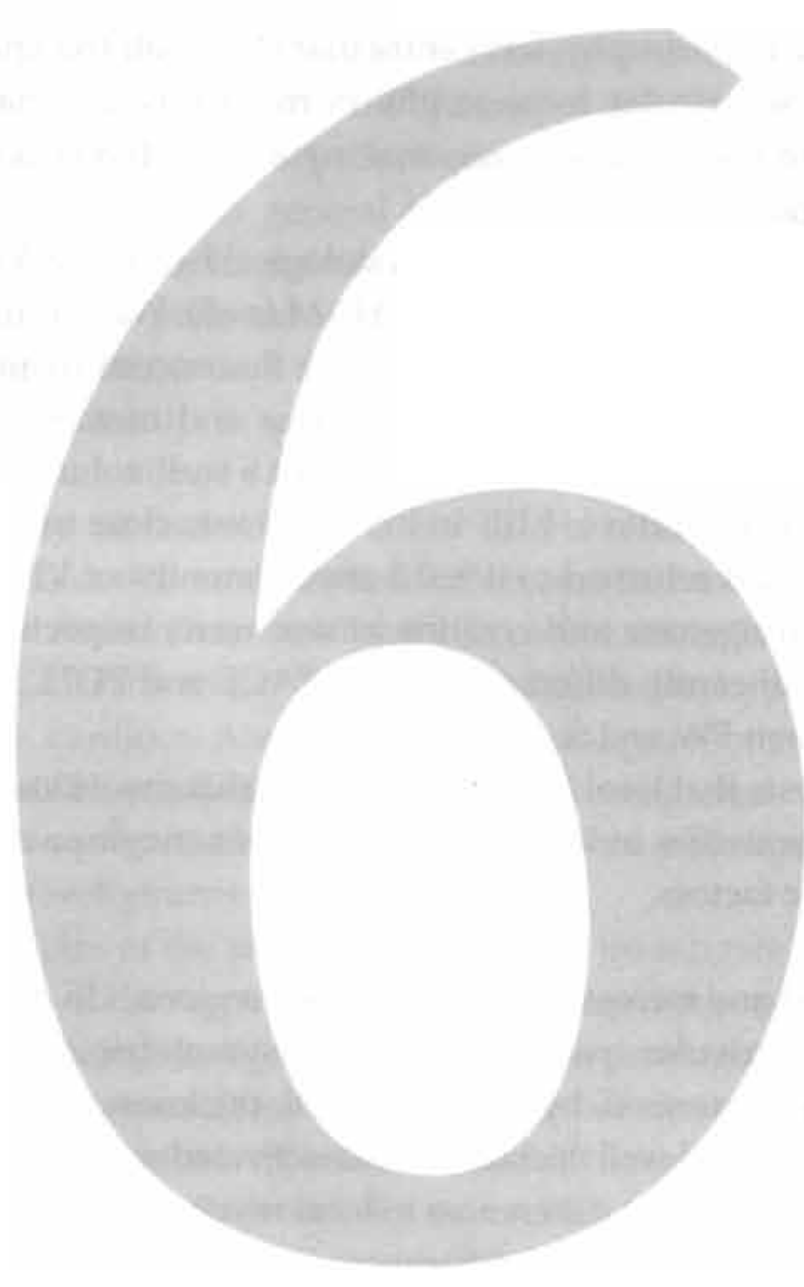

\section{REGIONAL BLOOD FLOW AND ACTIVITY OF METABOLIC ENZYMES IN CANINE HEARTS WITH PACING INDUCED ASYMMETRIC HYPERTROPHY}

M.F.M. van Oosterhout, F.W. Prinzen, J.F.C. Glatz, A.M.J. Rousseau, W.Y.R. Vanagt, T. Arts, R.S. Reneman

Cardiovascular Research Institute Maastricht (CARIM), The Netherlands

(Submitted) 
Background - Chronic ventricular pacing (VP) leads to asymmetric hypertrophy, left ventricular (LV) wall thickness being decreased in early and increased in late activated regions. Ventricular hypertrophy is frequently associated with diminished perfusion and altered metabolism. We investigated the changes in regional myocardial blood flow (MBF) and metabolic enzyme activities due to VP induced asymmetric hypertrophy.

Methods and Results - Experiments were performed on 3 groups of dogs: PACE (VP atphysiological heart rate, VP site: LV free wall, $n=8$ ); POH (pressure overload hypertrophy, by aortic constriction, $n=4)$ and SHAM ( $n=5$ ). Wall volume in the LV free wall (FW) and septum (S) was determined echocardiographically and NBF with fluorescent microspheres. Myocyte diameter and number of capillaries were determined with histological techniques and metabolic enzyme activities using biochemical techniques. In PACE animals FW wall volume did not thange but $\mathrm{S}$ wall volume increased by $39 \pm 13 \%$. Myocyte diameter was $18 \pm 7 \%$ larger in S than in FW. The ratio of MBF n FW and S was close to one during baseline sinus rhythm, decreased to $0.7 \pm 0.5$ after 15 min of VP and returned to $0.9 \pm 0.3$ after 6 months of VP. Activities of fructose-6-phosphate kinase, citrate synthase, lactate dehydrogenase and creatine kinase were, respectively, $52 \%$, $32 \%, 17 \%$ and $17 \%$ lower in PACE than in SHAM and not significantly different between PACE and POH. In none of the groups enzyme activities were significantly different between FW and S.

Conclusions - The uniform MBF distribution after chronic VP suggests that local hypertrophy normalizes workload per unit of tissue volume. The uniformly decreased metabolic enzyme activities in VP hearts indicate that enzyme activities are not determined by local hypertrophy but probably by systemic factors.

A ventricular pacing, creates regional differences in workload in the left ventricular (LV) wall, i.e. reduced workload in early activated regions and increased workload in late activated regions. As a consequence myocardial blood flow and oxygen uptake are reduced in early activa- ted and increased in late activated regions. ${ }^{1.2}$ In dogs chronic ventricular pacing leads to asymmetric hypertrophy, characterized by reduced wall thickness in early- and increased wall thickness in late-activated regions. ${ }^{3}$ These data indicate that differences in local myocardial growth can be regulated by local differences in myocardial workload. 
It is unknown, however, whether perfusion and metabolism also show local differences in the asymmetrically hypertrophied hearts. Such changes seem possible because in hearts with general LV hypertrophy various derangements occur. In global LV hypertrophy, especially when induced by pressure overload $(\mathrm{POH})$, the formation of new capillaries is less than the degree of myocardial growth. Especially in pressure overload hypertrophy, this leads to diminished vasodilatory capacity and increased susceptibility to ischemia, mainly in the endocardial layers. ${ }^{410}$ Moreover, modifications in myocardial metabolism like decreased Creatine Kinase (CK) and Lactate Dehydrogenase (LDH) activity ${ }^{11-18}$ have been observed in hypertrophic myocardium. Alternatively, such changes in metabolism and blood flow might be the consequence of changes in systemically acting factors, often accompanying the development of hypertrophy.

The aim of the present study was to investigate to what extent local hypertrophy, induced by long term ventricular pacing, leads to local changes in the degree of capillarization, perfusion and activity of metabolic enzymes. In case of the metabolic enzymes, a comparison was made between their activity in various parts of the asymmetrically hypertrophic ventricles, of normal hearts and of hearts with pressure overload hypertrophy (POH), as induced by aortic constriction. The $\mathrm{POH}$ group served as a positive control, displaying the changes in globally hypertrophied myocardium. To this purpose regional hypertrophy (2D echo) and blood flow (fluorescent microspheres) were measured in the early activated LV free wall and the late activated septum of dogs paced at the LV free wall for 6 months (PACE) and in sham operated controls (SHAM). These measurements were performed at the moment of implantation of the pacemakers and before the termination after the 6 months study period. Post mortem, tissue samples from the LV free wall and the septum were taken for analysis of myocyte diameter, collagen content and the activity of metabolic key enzymes. Similar post mortem measurements were performed in samples taken from hearts with $\mathrm{POH}$.

\section{METHODS}

Animal handling was performed according to the Dutch Law on Animal Experimentation (WOD) and the Guide for the Care and Use of Laboratory Animals published by the US National Institutes of Health (NIH Publication No. 85-23, revised 1996). The protocol was approved by the Experimental Animal Committee of the Maastricht University. Experiments were performed on dogs 
divided into three groups: dogs chronically paced from the LV free wall (PACE, $n=8$ ), sham operated dogs (SHAM, $n=5$ ) and dogs with pressure overload LV hypertrophy ( $\mathrm{POH}, \mathrm{n}=4)$.

\section{Implantation procedure PACE and SHAM groups}

Adult mongrel dogs of either sex were premedicated by an intramuscular injection of a mixture of acepromazine $0.2 \mathrm{mg} / \mathrm{kg}$, atropine $0.1 \mathrm{mg} / \mathrm{kg}$ and oxycodon $2 \mathrm{mg} / \mathrm{kg}$. Anesthesia was induced with Thiopental, $15 \mathrm{mg} / \mathrm{kg} \mathrm{IV}$, and maintained by ventilation with Halothane $(0.75-1.5 \%)$ in a $1: 2$ mixture of $\mathrm{O}_{2}$ and $\mathrm{N}_{2} \mathrm{O}$. Under sterile conditions the thorax was opened and pace leads were implanted in the right atrial cavity (Medtronic capsure sp 4423 lead) and in the epicardium of the free wall of the left ventricle, $1 \mathrm{~cm}$ below the base (Medtronic 4951M unipolar myocardial ventricular lead). In 8 dogs (PACE group, weight $28.9 \pm 9.5 \mathrm{~kg}$ ) a pacemaker (Medtronic Synergist H7027, H7071, Elite II or Thera DR 7941) was implanted. In 5 dogs (SHAM group, weight $24.4 \pm 2.3 \mathrm{~kg}$ ) no pacemaker was implanted, but for assessment of the short-term effects of pacing, the leads were temporarily connected to a pacemaker. A dual tip micromanometer catheter (Sentron) was introduced through the left femoral artery to measure LV cavity and ascending aortic pressure and to inject fluorescent microspheres for blood flow measurements into the left ventricle through its multiple side-hole end. In the right femoral artery a sample catheter was positioned to collect reference blood samples. Microsphere injections were made under baseline conditions and 15 minutes after ventricular pacing was initiated.

\section{Pace and echo protocol}

In the PACE group, ventricular pacing was started approxi- mately two weeks after implantation. The heart was stimulated at its own rhythm by AV sequential pacing (DDD-mode, upper rate 175 beats $/ \mathrm{min}$ ). The A-V stimulation interval was $25 \mathrm{~ms}$ to ensure complete ventricular capture. Proper pacemaker function was checked regularly.

Two-dimensional echocardiographic images of the left ventricle were made, at 0 and 6 months after onset of pacing in the PACE group and at the same time points in the SHAM group. Animals were slightly sedated with a mixture of acepromazine $(0.2$ $\mathrm{mg} / \mathrm{kg}$ ) and oxycodone (1 $2 \mathrm{mg} / \mathrm{kg}$ ). Parasternal short axis crosssectional images were taken, taking care that the LV appeared as circular as possible and that the tip of the papillary muscles and the pacing lead were visible. With these landmarks visible in each echo image it was possible to correlate the site of tissue collection at termination with the echographic image.

\section{Terminal procedure}

Six months after the implantation procedure the dogs of the PACE and SHAM groups were operated upon again, using the same anesthetic procedure and with the catheters introduced as during implantation. Measurements of the various parameters were performed with the pacemaker still functioning (in the PACED group), at the end of at least 15 minutes of temporary pacing (SHAM group), and $15 \mathrm{~min}$ after the pacemaker had been switched off (SHAM and PACE groups). After all measurements had been completed the heart was arrested in diastole by perfusion with ice-cold $\mathrm{CdCl}_{2}(0.1 \mathrm{M})$. The heart was quickly removed, the left and right ventricles were separated and weighed and transmural samples were taken from the LV free wall, at or near the pacing site, 
and from the septum opposite to that site. Non-muscular structures like fat and vessels at the epicardium, and chordae tendinae were removed. Each transmural sample was divided into two layers by midline dissection to obtain a subepicardial and subendocardial sample. For subsequent biochemical analysis samples where quickly immersed in liquid nitrogen and stored at $-80^{\circ} \mathrm{C}$. For microsphere analysis samples were weighed and stored at $-20^{\circ} \mathrm{C}$ together with the reference blood samples. Samples were also taken for analysis of myocyte thickness and collagen fraction as described before.

\section{Capillary to fiber ratio}

In transmural samples, taken from the LV free wall and septum, and embedded in paraffin, cardiomyocyte and capillary basement membranes were stained on $2 \mathrm{~mm}$ thick sections by the Jones silver methamine method. The capillary to fiber ratio was determined on 10 randomly taken fields in one section. The total number of cardiomyocytes and capillaries was counted using a 10 by 10 grid with border correction at a magnification of $400 \times$. $^{1.20}$

\section{Pressure overload hypertrophy (POH)}

In the POH group, $\mathrm{POH}$ was induced by aortic banding in puppies according to Nakano et al. ${ }^{2 i}$ In brief, under general anesthesia in 8-week-old puppies a slightly constricting ligature was placed around the ascending aorta to induce pressure overload. About 12 months later, when stabile LV hypertrophy had developed, the dogs were killed and the hearts removed; the left and right ventricles were weighed and tissue samples for biochemical and histological analysis were taken as described above.

\section{Analysis of echo images}

In an off line analysis, regional myocardial wall volume was estimated from the echo images as described in detail previously.' In brief, epicardial and endocardial contours were marked in a digitized 2D-echo image of the LV short axis at enddiastole. The contour points were converted to a polar representation with the center of the LV cavity as origin, and inner and outer radii were determined by fitting the original epicardial and endocardial contourpoints to a model with a limitation of the highest circular frequency to the fourth circular harmonic. The sector areas were defined by the endocardial and epicardial borders of the myocardial wall between the papillary muscles (LV free wall sector) and of the septal myocardial wall, opposite to the LV free wall sector (septal sector). Regional wall volume was defined as the product of the sector area and the mean LV radius, a measure of general dilatation.

\section{Analysis of blood flow}

The fluorescent microsphere method has been validated in short-term canine experiments.". Recently we showed that in chronic experiments fluorescent microspheres are superior to radioactive microspheres. Microspheres with blue, blue-green, yellow-green, orange, red or crimson fluorescent labels (polystyrene, $15.5 \mu \mathrm{m} \pm 2 \%$, Molecular Probes, Eugene, OR), were injected into the left ventricle. For each determination about $3 \cdot 10^{6}$ microspheres (suspended in 10\% dextran with $0.05 \%$. Tween 80 ) with a specific label were injected. Reference blood samples were taken from the femoral artery at a rate of $10.3 \mathrm{~m} \cdot \mathrm{min}^{-1}$ using a calibrated Harvard suction pump. Withdrawal of blood started $5 \mathrm{~s}$. 
before injection of the microspheres and was continued for at least $1 \mathrm{~min}$ after microsphere injection. Microspheres were isolated from myocardial samples by tissue digestion and subsequent centrifugal sedimentation. ${ }^{2}$ Fluorescence was determined using fluorimetry as described previously. ${ }^{n}$ Myocardial blood flow $\left(\mathrm{Q}_{i}, \mathrm{ml} / \mathrm{min} / \mathrm{g}\right)$ was calculated as:

$$
\mathrm{Q}_{i}=\mathrm{q}_{\text {ref }}\left(\mathrm{I}_{i} / \mathrm{I}_{\mathrm{ref}}\right) \mathrm{W}
$$

where $\mathrm{q}_{\mathrm{ref}}$ is withdrawal speed of the arterial reference sample in $\mathrm{ml} / \mathrm{min}, \mathrm{I}_{i}$ and $\mathrm{I}_{\mathrm{ref}}$ are the fluorescence intensity in sample $i$ and the reference sample, respectively, and $W$ is sample weight. The tissue weights determined post mortem were used for the flow measurements during the termination procedure $\left(\mathrm{W}_{i, 6}\right)$. In order to determine sample weight during the implantation procedure $\left(\mathrm{W}_{i, 0}\right)$ the site of tissue collection at termination was matched with the echographic image by use of echographically and anatomically well defined landmarks such as the papillary muscles and the pacing lead. $W_{i, 0}$ was estimated according to

$$
\mathrm{W}_{i, 0}=\mathrm{W}_{i, 6} \times\left(\mathrm{V}_{\mathrm{t}, 6} / \mathrm{V}_{\mathrm{t}, 0}\right)
$$

where $V_{t, 6} / V_{t, 0}$ is the ratio of the echocardiographically determined myocardial tissue volume at the end and the beginning of the study period, respectively.

\section{Biochemical assays}

Activities of individual metabolic enzymes were measured spectrophotometrically at $37^{\circ} \mathrm{C}$ in supernatants of sonicated muscle homogenates. Citrate synthase (CS) was assayed by the method of

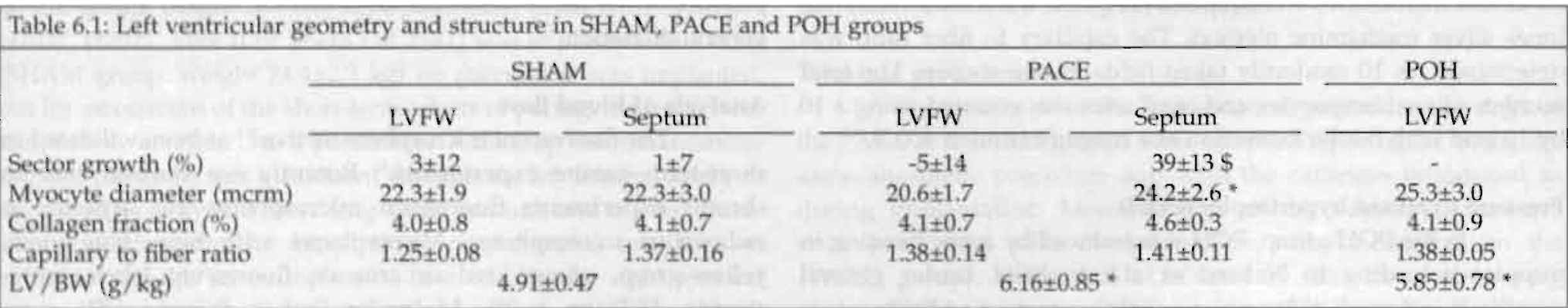

Table 6.1: Left ventricular gcometry (2D echo) and structure (histology) in dogs that were sham operated (SHAM-group), paced for6 months (PACE-group), and had pressure overload laypertroplry ( $\mathrm{POH}$-group). Sector growth: \% change in wall sector oolume as compared with the time of implantation (baseline) zoas determined in the LV free wall (LVFW) and septum, \$: p<0.05 compared to baseline eclto. Post mortem transmuclear myocyte diameter, collagen fraction and capillary to fiber ratio was determined in LVFW and septum of these dogs as well as the LV/body weight ratio (LV/BW). *: p<0.05 vs to sham, Values are means $\pm S D$. 
Shepherd and Garland," fructose-6-phosphate kinase (FPK) activity by the method of Ling et al., ${ }^{24}$ and 3-hydroxy-acyl-CoA dehydrogenase (HAD) according to Bergmeyer et al." Lactate dehydrogenase (LDH) activity (pyruvate as substrate, Boehringer Mannheim, FRG) and creatine kinase (CK) activity (NAC-activated CK test from Merck Diagnostica) were both assayed using standard test kits. All activities are expressed per $\mathrm{mg}$ of total protein. Protein content was determined by the Bicinchoninic acid (BCA) assay (Pierce) with bovine serum albumin as the standard.

\section{Statistical analysis}

Differences within the groups were evaluated for statistical significance with the Wilcoxon signed rank test and between the groups with the Mann-Whitney $U$ test. The level of significance was set at $p<0.05$. Data are presented as mean values with standard deviations (SD).

\section{RESULTS}

\section{Structural changes}

In the PACE and POH groups significant global LV hypertrophy was present, LV/body weight (LV/BW) ratios being respectively $25 \%$ and $19 \%$ higher than in the SHAM group (Table 6.1). In the PACE group, echocardiographic measurements showed a $39 \pm 13 \%$ increase of the septal wall sector volume and no significant change in the LV free wall sector volume as compared witb the time of implantation (baseline). The ratio of free wall and septum sector volume decreased significantly by $36 \pm 14 \%$ compared with baseline (Table 6.1). Histological analysis showed that in the PACE group the diameter of the septal myocytes was $18 \pm 7 \%$ larger than in the $L V$ free wall. Septal myocyte diameter was $9 \%$ larger in the PACE than in the SHAM group, whereas LV free wall myocyte diameter was not significantly different in the PACE and SHAM groups. LV free wall myocyte diameter was $23 \%$ and $13 \%$ larger in the $\mathrm{POH}$ group than in the PACE and in the SHAM group, respectively (Table 6.1). Despite the thicker myocytes in the septum of the PACE group and in the $\mathrm{POH}$ hearts, the number of capillaries per fiber in these regions was not different from that in the SHAM group and in the LV free wall of the PACE group. Collagen fraction was not significantly different between and within groups (Table 6.1).

\section{Regional myocardial blood flow (MBF)}

During the implantation procedure $\mathrm{MBF}$ was not significantly different between the PACE and SHAM groups. MBF during sinus rhythm was not significantly different between the $L V$ free wall and septum (Table 6.2), indicating uniform blood flow distribution. The unifor- 


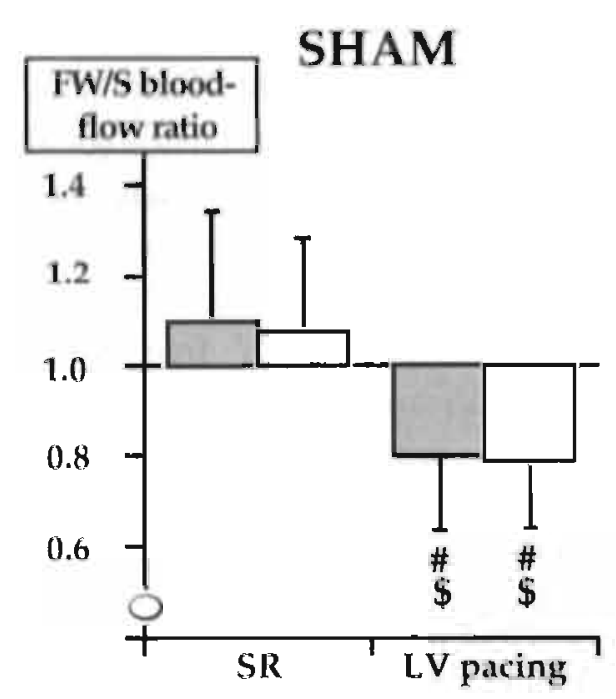

mity of blood flow was further supported by values of the LV free wall to septum (FW/S) blood flow ratio being close to unity (Figure 6.1, left panel). The effect of $15 \mathrm{~min}$ of temporary pacing was similar in both groups. MBF was significantly reduced in the early-activated $L V$ free wall compared with sinus rhythm and was significantly lower than in the septum (Table 6.2). Changes in MBF due to pacing in the late-activated septum were not significant. These changes in blood flow resulted in an approximately $20 \%$ decrease of the FW/S blood flow ratio (Figure 6.1, left panel).

\section{PACE}

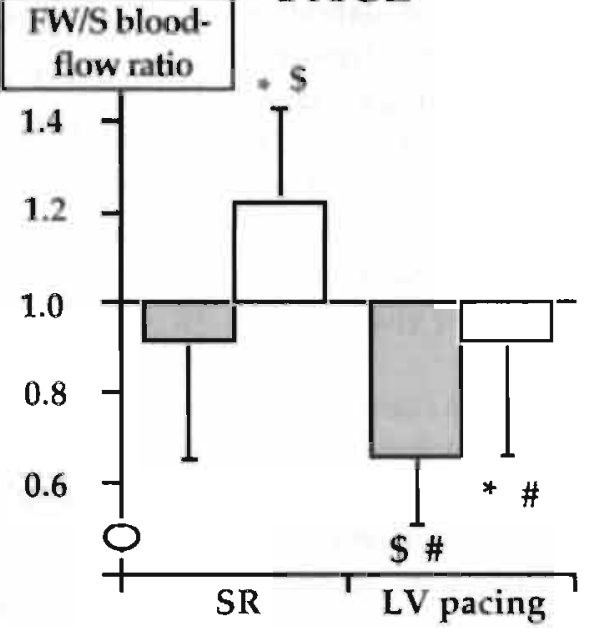

Fig 6.1

The ratio of blood flow in LV free wall and septum (FW/S blood flow natio) during simus ritythm (SR) and IV free wall pacing (LV pacing) in the SHAM (left panel) and PACE (right panel) groups during implantation (hatched bars) and during termination after 6 months (open bars). \#: $p<0.05$ Pacing vs. $S R, *: p<0.05$ termination vs. implantation. \$: significantly different from unity. 
not significantly different (Table 6.2). Moreover, the FW/S blood flow ratio was higher at termination than at implantation, both during sinus rhythm and during pacing. After 6 months of pacing the FW/S blood flow ratio was not significantly different from unity whereas during sinus rhythm this ratio was significantly above unity (Figure 6.1, right panel).

\section{Metabolic enzyme activities}

At first, in a subset of samples from PACE $(n=4)$

\begin{tabular}{|c|c|c|c|c|}
\hline \multicolumn{5}{|c|}{ Table 6.2: Myocardial blood flow } \\
\hline & \multicolumn{2}{|c|}{ SHAM } & \multicolumn{2}{|c|}{ PACE } \\
\hline & LVTW & Septum & LVFW & Septum \\
\hline $\begin{array}{l}\text { Implantation } \\
\text { sinus rhythm } \\
\text { Pacing }\end{array}$ & $\begin{array}{l}0.82 \pm 0.32 \\
0.67 \pm 0.13\end{array}$ & $\begin{array}{l}0.76 \pm 0.26 \\
0.86 \pm 0.18 \$\end{array}$ & $\begin{array}{l}0.79 \pm 0.52 \\
0.51 \pm 0.31 *\end{array}$ & $\begin{array}{l}0.84 \pm 0.46 \\
0.74 \pm 0.34\end{array}$ \\
\hline $\begin{array}{l}\text { Termination } \\
\text { sinus rhythm } \\
\text { Pacing }\end{array}$ & $\begin{array}{l}0.69 \pm 0.06 \\
0.76 \pm 0.22\end{array}$ & $\begin{array}{l}0.65 \pm 0.12 \\
0.98 \pm 0.27 \$\end{array}$ & $\begin{array}{l}0.58 \pm 0.33 \\
0.54 \pm 0.30\end{array}$ & $\begin{array}{l}0.47 \pm 0.24 \\
0.64 \pm 0.44\end{array}$ \\
\hline
\end{tabular}

Table 6.2 Myocardial blood flow ( $\mathrm{ml} / \mathrm{min} / \mathrm{g}$ ) in transmural samples taken from the septum and left ventricular free wall (LVFW) during simus rhythm and pacing at implantation and at termination in dogs that were sham operated (SHAM) and paced for 6 months. (PACE). mean $\pm S D,{ }^{*} p<0.05$ sinus rhythm vs Pacing, same site; \$ p<0.05 LVFW os Septum and SHAM $(\mathrm{n}=3)$ animals, enzyme activity was analyzed in epicardial and endocardial samples separately. Because no consistent transmural differences in enzyme activities were found (data not shown), the data for epicardial and endocardial enzyme activity were pooled for these experiments and compared with the data derived from transmural samples from the other experiments. In none of the groups a significant difference was found between samples from the LV free wall and the septum in the activity of each of the metabolic enzymes, in the wet weight/dry weight ratio and in the protein content (Table 6.3). The values of most of these variables, however, were significantly different between the three groups (Table 6.3). Figure 6.2 presents the data on enzyme activities and wet weight/dry weight ratios after pooling values from septum and free wall. Enzyme activity of FPK was respectively $52 \%$ and $62 \%$ lower in PACE $(p<0.05)$ and $\mathrm{POH}(\mathrm{p}<0.05)$ than in SHAM. Less pronounced decreases were found for the activities of CS, CK and LDH: $32 \%$, $17 \%$ and $17 \%$, respectively, in the PACE group (all p<0.05) and $24 \%(\mathrm{p}<0.05), 9 \%$ and $7 \%$ (both ns), respectively, in the $\mathrm{POH}$ group. HAD activity was not significantly different betwcen the threc groups. Because the protein content (mg protein/mg wet weight) was significantly (about 10\%) 
lower in the pace and the POH than in the SHAM group (Table 6.3) enzyme activity per gram wet weight was even lower in PACE and POH than in SHAM (data not shown). The wet weight/dry weight ratio was significantly elevated (by $\sim 25 \%$ ) in the PACE compared to the SHAM group (Table 6.3, Fig. 6.2).

\section{DISCUSSION}

The present stidy demonstrates that ventricular pacing induces reginal hypertrophy. This regional hypertrophy is characerized by an increase in myocyte diameter, but does no lead to a regional increase in the number of capillaries per fiber. Nevertheless, the distribution of MBF (expressed per unit of volume) that is nonuniform acutely after onset of pacing, becomes uniform within 6 months of ventricular pacing. Assuming

\begin{tabular}{|c|c|c|c|c|c|c|}
\hline & $w 4 x^{2}+3 x$ & & & & 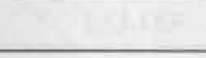 & \\
\hline & LVFW & Septum & LVFW & Septum & LVFW & Septum \\
\hline CK & $21.96 \pm 3.96$ & $22.76 \pm 2.88$ & $17.71 \pm 2.15$ & $19.30 \pm 2.36$ " & $20.93 \pm 2.54$ & $19.70 \pm 1.93$ \\
\hline CS & $0.25 \pm 0.03$ & $0.24 \pm 0.03$ & $0.18 \pm 0.03^{*}$ & $0.17 \pm 0.05^{*}$ & $0.19 \pm 0.05^{*}$ & $0.21 \pm 0.03 *$ \\
\hline HAD & $0.14 \pm 0.02$ & $0.12 \pm 0.01$ & $0.13 \pm 0.03$ & $0.11 \pm 0.04$ & $0.13 \pm 0.01$ & $0.14 \pm 0.01$ \\
\hline LDH & $3.50 \pm 0.42$ & $3.56 \pm 0.28$ & $2.92 \pm 0.28$ * & $2.93 \pm 0.34 *$ & $3.36 \pm 0.58$ & $3.16 \pm 0.41$ \\
\hline FPK & $0.30 \pm 0.10$ & $0.27 \pm 0.08$ & $0.17 \pm 0.11$ & $0.12+0.10^{*}$ & $0.12 \pm 0.10^{*}$ & $0.09 \pm 0.04$ * \\
\hline Prot & $0.19 \pm 0.01$ & $0.19 \pm 0.08$ & $0.16 \pm 0.02^{*}$ & $0.16 \pm 0.02$ * & $0.16 \pm 0.01^{*}$ & $0.17 \pm 0.01$ * \\
\hline Ww/Dw & $4.29 \pm 0.24$ & $3.80 \pm 0.51$ & $5.03 \pm 0.62^{*}$ & $4.81 \pm 0.55^{*}$ & n.d. & n.d. \\
\hline
\end{tabular}

Table 6.3 Enzyme activity (U/microg protein, means \pm SD) of creatine kinase (CK), citrate synthase (CS), 3-hydroxy-acyl-CoA dehydrogenase (HAD), iactate deludrogenase (LDH) and fructose-6-phosphate kinase (FPK) and protein content (Prot, mg/mg wet weight) and the wet weight / dry weight (Ww/Dw) ratio (see legend of Fig. 6.1 for explanation) in paced (PACE), sham openated and pressure overload hypertrophy (POH) dogs. Transmural samples were taken from the leff ventricular free wall (LVFW) and ventricular septum. ${ }^{*} P<0.05$ vs sham. nd. $=$ not determined 


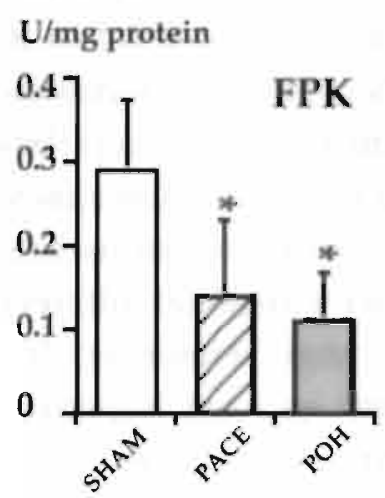

U/mg protein

U/mg protein

$\mathrm{U} / \mathrm{mg}$ protein
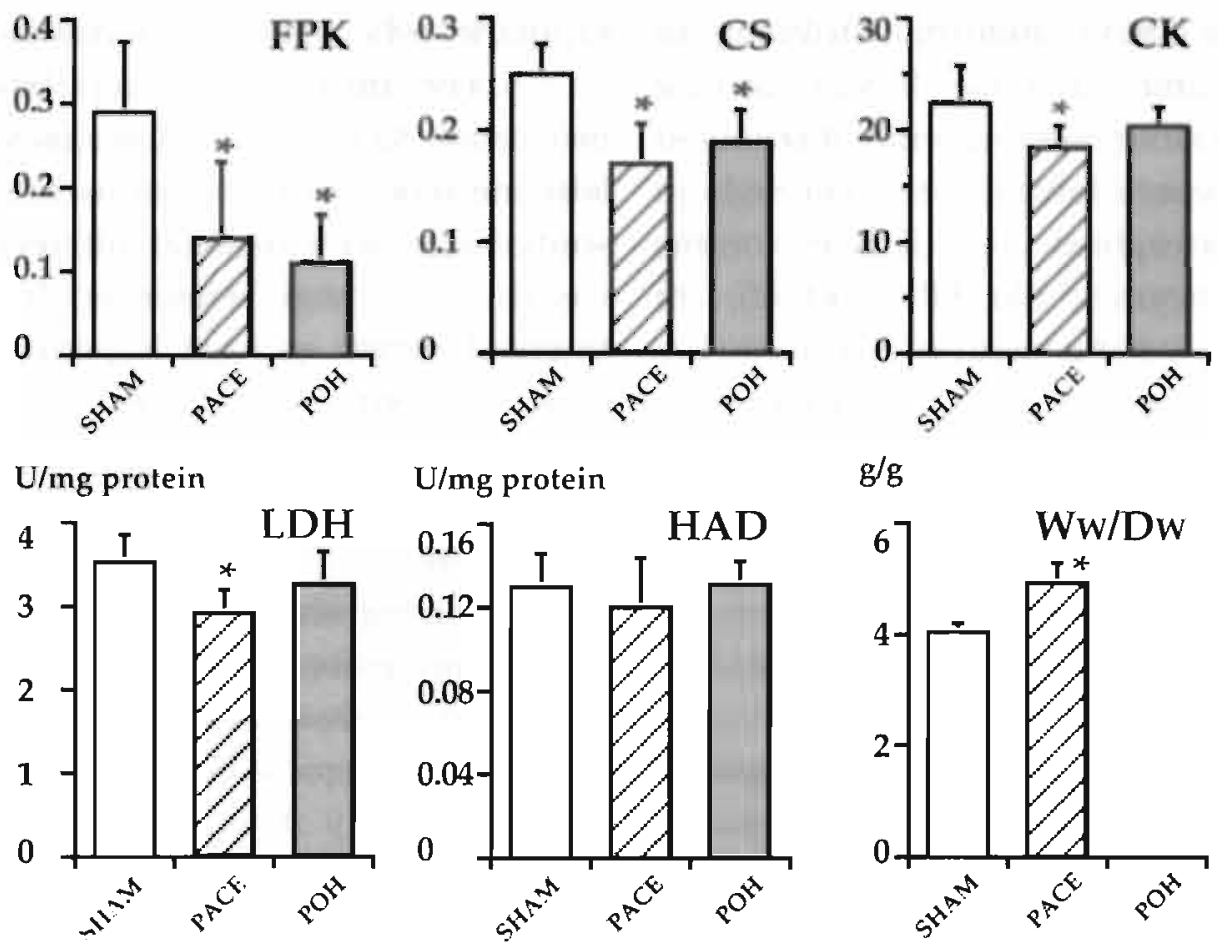

U/mg protein

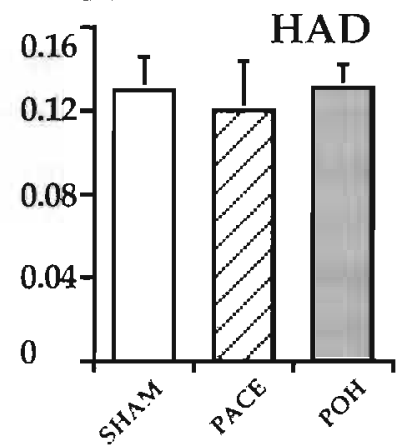

$\mathrm{g} / \mathrm{g}$

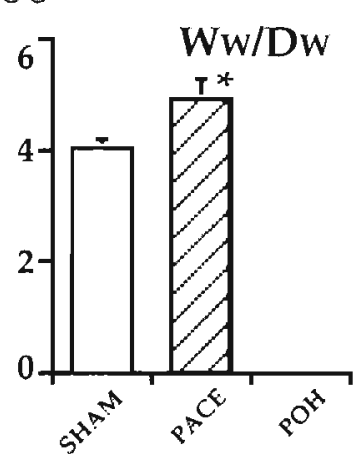

Fig 2: Activity (U/mg protein, mean \pm SD) of creatine kinase (CK), citrate synthase (CS), 3-hydroxy-ncyl-CoA dehydrogenase (HAD), laclate dehydrogenase (LDH) and fructose-6-phosphate kinase (FPK) in LV tissue. Data are average of LV free wall and septum in the SHAM (open bars, $n=5$ ), PACE (black bars, $n=7)$ and POH (hatched bars, $n=4)$ groups. Ww/Du: the wet weight/dry weight ratio, not determined (n.d.) in the POH group. ${ }^{*}:$ p<0.05. vs SHAM. 
that myocardial mechanical load is proportional with blood flow, this finding suggests that the local hypertrophy process has led to a more uniform distribution of load per unit of tissue volume within the LV wall. Also the activities of metabolic enzymes are uniformly distributed in the asymmetrically hypertrophied heart. Throughout the asymmetrically hypertrophied LV wall, however, the activities of metabolic enzymes, like FPK and CS, are significantly reduced and water content is increased. The values of enzyme activity are similar in the paced hearts and in ventricles with $\mathrm{POH}$. These findings indicate that changes in the activity of metabolic enzymes are not directly related to the regional degree of hypertrophy, but rather to systemic factors.

\section{Capillarization}

In the hypertrophied region of the chronically paced hearts and in $\mathrm{POH}$ hearts the capillary to fiber ratio was unchanged whereas myocyte diameter was increased. This indicates that in these regions capillary density (capillaries per unit volume) is lower than in the nonhypertrophied region of the paced hearts and in the myocardium of SHAM animals. Therefore, the well-known notion that $\mathrm{POH}$ is accompanied by reduced capillari- zation $^{\text {th }}$ and vasodilatory capacity ${ }^{\mathrm{WI}}$ appears to be due to the inability at the local level to increase the number of capillaries when cardiomyocytes increase their diameter.

The finding that capillaries do not grow in proportion to myocytes does, most likely, not affect the baseline blood flow values as measured in the present study, because the regional differences are well within the vasodilatory range. However, like in globally hypertrophied ventricles, the hypertrophied region may be more susceptible to ischemia under more compromising hemodynamic circumstances.

\section{Blood flow}

During short-term pacing blood flow in transmural samples located close to the pacing site decreased as compared to sinus rhythm. This is in agreement with findings in subepicardial samples in previous studies. ${ }^{1.2}$ In an earlier study it was shown that the distribution of blood flow is proportional to workload. The initially nonuniform distribution of blood flow disappeared with longer lasting ventricular pacing, as evidenced by a FW/S blood flow ratio not significantly different from unity after 6 months of pacing (Fig. 6.1). Therefore, the distribution of local load is probably more uniform during longer lasting 
than during acute ventricular pacing. This greater uniformity on the long run is most likely caused by the development of local septal hypertrophy. The degree of nonuniformity of blood flow diring acute ventricular pacing is similar to the degree of hypertrophy in the late activated septum. Therefore, the increase in volume in the late activated septum decreases workload per unit volume to the level in early activated regions.

Arter six montns or ventricular pacing the return to sinus rhy thm causes a nonuniform blood flow distribution with a FW/S blood flow ratio above unity. The explanation for this observation may be the following. During sinus rhythm the distribution of workload per sector of the LV wall is uniform, but during 6 months of pacing the septal sectors have hypertrophied so that per unit volume workload is lower in the septum than in the LV free wall.

\section{Regional differences in activity of metabolic enzymes}

In the asymmetrically hypertrophied hearts of chronically paced dogs not only blood flow but also enzyme activities are distributed uniformly, a finding that may endorse a uniform distribution of myocardial metabolism in the chronically paced heart. Enzyme activities in the various (hypertrophied and nonhypertrophied) regions of the chronically paced hearts are decreased as compared to SHAM animals in a way similar to that in ventricles with $\mathrm{POH}$. These findings indicate that myocardial enzyme activity is not directly dependent on the degree of local hypertrophy, Other factors, potentially at a more systemic level, may be responsible for the local changes in enzyme activity. This idea is supported by the findings in other studies. Smith et all ${ }^{20}$ showed that systemic hypertension, inducing LV hypertrophy without RV hypertrophy, is associated with increased tissue levels of myocardial $\mathrm{LDH}$ and decreased levels of CK in both ventricles. In another study, enzymatic changes were reported in hypertensive rabbits without cardiac hypertrophy. ${ }^{27}$

Systemic reactions may have been evoked by the reduction in ventricular function due to ventricular pacing. ${ }^{328,29}$ Such hemodynamic changes may lead to changes in circulating hormones and sympathetic activity, as indicated by the increased myocardial tissue contents of norepinephrine in chronically paced dogs. In rabbits a hemodynamic intervention, like aortic banding, increased plasma renin activity as well as norepinephrine content. This was associated with a transient sodium retention. ${ }^{31}$ 
Sodium retention may explain the elevated myocardial water content in the PACE group, evidenced by lower protein content and higher wet weight/dry weight ratio. Therefore, myocardial enzyme concentrations might be regulated by substances like catecholamines and angiotensin. The similar decrease in myocardial enzyme activity in the PACE and POH group may be explained by similar systemic responses to pressure overload and ventricular pacing.

\section{Global changes in metabolic enzyme activities}

Decreased total CK activity has also been found in studies on hypertrophic hearts. ${ }^{910}$ Reported changes in myocardial LDH activity due to hypertrophy range from a decrease, ${ }^{12}$ like in the present study, to unchanged activity, ${ }^{11}$ or even increased activity. ${ }^{1520 x}$ The finding of decreased CS activity is comparable to that of Gibbs et al. ${ }^{33}$ in $\mathrm{POH}$ in rabbit hearts but not with the unchanged CS activity in patients with aortic stenosis."

The knowledge on activity of HAD and FPK in hypertrophic canine hearts is limited. Therefore, the findings on the decrease in FPK and the lack of change in $\mathrm{HAD}$ in pacing and pressure overload induced hypertrophy is interesting. These findings are more or less surprising, because studies on uptake of labeled glucose and fatty acids showed that in hypertrophic hearts utilization of glycolytic pathways is increased and utilization of fatty acids is often impaired. ${ }^{\text {suszos }}$ The discrepancy between data obtained in these uptake studies and the enzyme activity data obtained in the present study (Figure 6.2) may be explained by the fact that enzyme activity is determined in vitro under optimal, artificial conditions. In contrast, the results obtained in uptake studies are also dependent on the metabolic regulation of the myocardium in vivo. Also differences in species, degree of hypertrophy and presence of heart failure may contribute to the discrepancy between the present study and the uptake studies. In a previous study with a similar design as the present study, 6 months of ventricular pacing was not associated with cardiac failure.

\section{Conclusions}

The findings in the present study show that the capillary density is lower in the hypertrophic than in the non-hypertrophic region of paced hearts. The uniform distribution of MBF after chronic ventricular pacing suggests that within the paced ventricle local hypertrophy 
normalizes load per unit of tissu volume. The uniform decrease of the activity of seveal metabolic enzymes indicates that the activity of hese enzymes is not determined by local hypertrophy lut that systemic factors may affect enzyme activity in pacid hearts.

\section{REFERENCES}

1. Prinzen FW, Augustijn CH, Arts T, Allessie MA Reneman RS. Redistribution of myocardial fiber strain and blood flow by asyndaronous activation. Am I Physiol. 1990;259:H300-H308

2. Delhaas T. Arts T, Prinzen FW Reneman RS. Regional fibre stress-fibre strain area as an. estimate of regional blood flow and oxygen demand in the canine heart. J Physiol (London). 1994:477.3:481-496.

3. Van Oosterhout MFM, Prinzen FW, Arts T; Schreuder JS, Vanagt WYR, Cleutjens IPM Reneman RS, Asynchrounous electrical activation induces asymmetrical hypertrophy of the left ventricular wall. Circulation. 1998-98:588-595.

4. Engelmann GL, Vitullo JC Gerrity RG. Morphometric analysis of cardiac hypertrophy during development, maturation, and senescence in spontaneously hypertensive rats. Circ Res. 1987;60:487-494.

5. Henquell L. Odoroff CL Honig CR. Intercapillary distance and capillary reserve in hypertrophied rat hearts beating in situ. Circ Res. 1977;41:400-408.

6. Rakusan K. Candiac growth response to chronic exercise. In: Tailor, AW, ed. Biochemistry of exercise. Human kinetic publishers, inc.,1990:113-121.

7. Rakusan K, Hrdina PW, Turek Z. Lakatta EG, Spurgeon HA Wolford GD. Cell size and capillary supply of the hypertensive rat heart: quantitative study. Basic Res Cardiol. 1984;79:389-395.

8. Tomanek RI, Schalk KA, Marcus ML. Harrison DG: Coronary angiogenesis during long-term hypertension and left ventricular hypertrophy in dogs. Circ. Res. $1989 ; 65 ; 352-359$

9. Snoeckx LHEH, Var der Vusse GJ, Van der Veen FH, Coumans WA Reneman RS. Recovery of hypertrophied rat hearts after global ischemia and reperfusion at different perfusion pressures. Eur J Physiol (Pfluegers Arch) 1989;413:303-312.

10. Hittinger L, Patrick T, Thara T, Hasebe N, Shen Y-T, Kalthof B, Shannon RP Vatner SE. Exercise induces cardiac dysfunction in both moderate, compensated and severe hypertrophy. Circulation. 1994,89:2219-2231.
11. Ingwall JS, Kramer ME, Fiter MA, Lorell BH, Shemin K, Cirossman W Allen PD. The creatine kinase system in normal and diseased human myocardium. N Engl I Med. 1985:313:1050-1054.

12. Laarse van der A, Vliegen HW, Nat van der KH, Hollaar L. Egas IM, Swier GPH Broek van den AICM. Comparison of myocardial changes between pressure induced hypertrophy and normal growth in the rat heart. Cardiovase Res. $1989,23,305,314$

13. Zhu Y.C. Zhu Y-Z, Spitznagel H. Gohlike P Unger T. Substrate metabolism. hormone interaction, and angiotensin- converting enxyme inhibitors in left ventricular hypertrophy. Diabetes. $1996 \% 45 \$ 599665$.

14. Rossi A Lortet S. Energy metabolism patterns in mammalian myocardium adapted. to chronic physiopathological conditions. Cardiovasc Res. 1994:31:163-171.

15. Bishop SP Altschuld RA. Increased glycolytic metabolism in cardiac hypertrophy and congestive failure. Am J Physiol. 1970,218:153-159.

16. Wittels B Spann Ir IF. Defective lipid metabolism in the failing heart. I Clin Invest. $1968: 47: 1787.17914$

17. Wambolt RB, Henning SL, English DR, Bondy GP Allard MF Regression of cardiac hypertrophy nomalizes glucose metabolism and left ventricular function during reperfusion. J Moll Cell Cardiol. 1997:29:939-948.

18. Massie BM, Schaefer S. Garcia J McKirnan MD, Schwartz GG. Wisneski IA. Weiner MW White FC. Myocardial high-energy phosphate and substrate metabolism in. swine with moderate left ventricular hypertrophy. Circulation. 1995;91:1814-1823,

19. Rakusan K. Flanagan ME, Geva T, Southern I Van Praagh R. Morphometry of human coronary sapillaries during norma! growth and the effect of age in left ventricular pressure-overload hypertrophy. Circulation. 1992;86:83-46.

20. 5abbah HN, Sharov' VG, Lesch MI Goldstein S. Progression of heart failure: a role for interstitial fibrosis. Mol Cell Biochem. 1995;147:29-34.

21. Nakano K, Corin Wi, Spann JF, Biederman RWW, Denslow S Carabello BA. Abnormal subendocardial blood flow ini pressure overload hypertrophy is associated with pacing-induced subendocardial dysfunction. Circ Res. $1989 ; 65:-1555-1564$

22. Van Oosterhout MFM, Willigers HMM, Reneman RS Frinzen FW. Fluorescent microspheres to measure organ perfusion: validation of a simplified sample processing technique. Am ] Physiol. 1995;269:H725-H733.

23. Shepherd D Garland PB. Citrate synthase from rat liver. Methods Enzymol. 1969;13:11-16.

24. Ling KH, Paetkau V, Marcus F Lardy HA. Phosphofructokinase in skeletal muscle, Methods Enzymol. 1966:9:425-429.

25. Bergmeyer HU. New York, NY: Academic Press, 1974 .

26. Smith SH, Kramer MF, Reis I, Bishop SF Ingwall jS. Regional changes in creatine kinase and myocyte size in hyperfensive and nonhypertensive cardiac hypertrophy. Circ Res. 1990;67:1334-1344.

27. Taegtmeyer H Overturf ML. Effects of moderate hypertension on cardiac function and metabolism in, the rabbit. Hypertension. 1988;11:416-426. 


\section{Chapter 6}

28. Rosenqvist M, Isaaz K, Botvinick EH, Dae MW, Cockrell J, Abbott JA, Schiller NB Griffin JC. Relative importance of activation sequence compared to atrioventricular synchrony in left ventricular function. Am J Cardiol. 1991,67:148. 156.

29. Rosenqvist M. Bergfeldt L. Haga Y, Rydén J. Rydén L. Owall A. The effect of ventricular activation sequence on cardiac performance during pacing. Pace. $1996 ; 19: 1279-1286$.

30. Lee MA, Dae MW, Langberg J, Griffin JC, Chin MC, Finkbeiner WE, O'Connell JW, Botvinick E, Scheinman MM Rosenqvist M. Effects of long-term right ventricular apical pacing on left ventricular perfusion, innervation, function and histology. J Am Coll Cardiol. 1994:24:225-232.

31. Whitlow PL Katholi RE. neurohumoral mechanisms in acute aortic coarctation in conscious and anesthetized dogs. Am J Phisiol. 1983-244:H614-621.

32. Laser A, Ingwall JS, Taan R, Reis L, Hu K, Gaudron P, Ertl G Neubauer S. Regional biochemical remodeling in non-infarcted tissue of rat heart post-myocardial infanction. I Mol Cell Cardiol. 1996;28:1531-1538.

33. Gibbs Cl. Wendt IR, Kotsanas G. Ross Young I Woolley G. Mechanical, energetic, and biochemical changes in long-term pressure overload of rabbit heart. Am I Physiol. 1990:259-H849. H859 


\section{VENTRICULAR PACING INDUCES REMODELING IN HYPERTROPHYING DOG HEARTS}

M.F.M. van Oosterhout', T. Arts', A.M.M. Muijtjens ${ }^{3,}$ R.S. Reneman' ${ }^{1}$ F.W. Prinzen'

Departments of Physiology', Biophysics', and Medical Informatics

Cardiovascular Research Institute Maastricht, Maastricht University and University Hospital, Maastricht, The Netherlands

(Submitted) 
Background - In normal hearts asynchronous electrical activation, induced by ventricular pacing (VP), causes diminished mechanical load in early-activated myocardial regions and enhanced mechanical load in late-activated myocardial regions. These conditions ultimately lead to reduced wall thickness in early- and increased wall thickness in lateactivated regions. In the present study we investigated whether VP induces a similar remodeling in hearts with pressure overload hypertrophy $(\mathrm{POH})$.

Methods and Results - POH was induced by aorta banding in puppies. At age 9 months 7 dogs were paced rate at the right ventricular (RV) apex at physiological heart for 6 months (POH-pace group). Four POH dogs served as control (POHcontrol group). 2D-echocardiography and X-ray marker detection were used to measure left ventricular (LV) wall volume and the relative changes in volume of $5 \mathrm{LV}$ wall sectors (apical and basal septum and anterior, posterior and lateral LV wall) between age 9 and 15 months. In the POH-control group. LV wall volume increased, varying from $27 \pm 9 \%$ in the apical septum to $30 \pm 5 \%$ in the lateral LV wall $(\mathrm{x} \pm \mathrm{s}$.d.). In the POH-pace group the increase in regional wall volume in 4 out of 5 sectors ranged from $31 \pm 16$ to $35 \pm 17 \%$. In the early-activated apical septum, however, this increase was significantly snnaller $(17 \pm 21 \%)$. In these hearts myocyte diameter was smaller in the apical septum than in the lateral LV wall. Chronic pacing did not further deteriorate the reduced LV function seen after acute pacing.

Conclusions - In hypertrophying hearts chronic pacing at the RV apex suppresses the development of hypertrophy in the early-activated apical septum. In contrast to normal hearts VP does not lead to excess hypertrophy in late-activated regions.

I

n normal canine hearts asynchronous electrical activation, as induced by ventricular pacing, causes regional differences in workload within the left ventricular (LV) wall, ${ }^{12}$ i.e. reduced workload in early- and increased workload in late-activated areas. These conditions ultimately lead to asymmetric hypertrophy, the LV wall becoming thinner in early- and thicker in lateactivated regions. ${ }^{3}$ These observations indicate that local myocardial load is an important determinant of local myocardial growth. It is as yet unknown whether ventricular pacing can also induce regional changes in myocardial mass in globally hypertrophied hearts. It may quite well 
be that the factors responsible for the induction of global LV hypertrophy, in such disorders as pressure overload, may dominate those involved in local myocardial growth. After all, it has been shown that the growth response to mechanical stimulation is less in hypertrophic than in nonhypertrophic hearts. ${ }^{4}$

Insight into the influence of ventricular pacing on remodeling of the LV wall, if any, is clinically relevant, because pacing is used as therapy in patients with hypertrophic obstructive cardiomyopathy (HOCM). In these patients the basal septum is thickened, which contributes to the dynamic outflow obstruction of the LV. Several studies showed that right ventricular (RV) apex pacing in patients with HOCM acutely improves the LV-aortic pressure gradient and that this improvement increases over time. ${ }^{57}$ Even after termination of chronic pacing the LV-aortic pressure gradient was less than before onset of pacing. The latter observation indicates that in these patients long term pacing has led to structural adaptations. Because during RV apex pacing the septum is earlyactivated and thereby mechanically unloaded, ${ }^{1,2}$ RV apex pacing may induce beneficial remodeling in hypertrophic hearts, i.e., suppression or reversal of hypertrophy in the septum.
In several studies it has been shown that ventricular pacing reduces acutely LV pump function. ${ }^{1.11}$ This reduction is not further affected during chronic ventricular pacing, at least in normal, non-hypertrophic canine hearts. ${ }^{3}$ Whether the latter is also the case in hypertrophic hearts is as yet unknown.

In the present study we investigated the effects of dual-chamber pacing on LV geometry in dogs with developing pressure overload hypertrophy $(\mathrm{POH})$ as induced by aortic banding. The hypothesis was tested that in LV hypertrophy pacing from the RV apex leads to selective suppression of wall hypertrophy of the earlyactivated septum.

Before and at various intervals during 6 months of $\mathrm{RV}$ apex pacing changes in global and regional LV geometry were assessed by $2 \mathrm{D}$-echocardiography and $\mathrm{X}$ ray marker analysis. Global LV function was characterized by LV pressure and aortic flow measurements. Post: mortem, myocyte diameter and collagen fraction were determined in the LV free wall and septum. Non-paced $\mathrm{POH}$ dogs were used as controls. 


\section{METHODS}

Animal handling was performed according to the Dutch Law on Animal Experimentation (WOD) and The European Directive for the Protection of Vertebrate Animals used for Experimental and other Scientific Purposes (86/609/EU). The protocol was approved by the Animal Experimental Committee of the Maastricht University.

\section{induction of pressure overload}

Eleven mongrel puppies, 2 months of age and weighing $7.0 \pm 0.8 \mathrm{~kg}$, underwent aortic banding according to Nakano et al. ${ }^{12}$ In brief, the dogs were premedicated by an intramuscular injection of a mixture of acepromazine $0.2 \mathrm{mg} / \mathrm{kg}$, atropine $0.1 \mathrm{mg} / \mathrm{kg}$ and oxycodon $2 \mathrm{mg} / \mathrm{kg}$. Anesthesia was induced with Thiopental, 15 $\mathrm{mg} / \mathrm{kg}$ IV, and maintained by ventilation with Halothane $(0.75-$ $1.5 \%$ ) in a $1: 2$ mixture of $\mathrm{O}_{2}$ and $\mathrm{N}_{2} \mathrm{O}$. The thorax was opened through the left fifth intercostal space and the aorta was exposed by pericardiotomy. A slightly constricting 5-mm-wide Mersilene ligature was placed around the ascending aorta just above the coronary arteries. In four animals this degree of aortic constriction was associated with a reproducible peak systolic pressure gradient of 5-10 mmHg between the LV and the descending aorta, in the other animals gradients were not determined during the banding procedure. After the induction of pressure overload, body weight and LV wall volume (by 2D-echocardiography, see below) were determined monthly. The second operation was postponed until body weight leveled off, which occurred at the age of $9 \pm 1$ months.

\section{Implantation procedure}

At the age of $9 \pm 1$ months the dogs were operated again. Anesthesia was induced and initially maintained as described above. At least one hour before determination of the hemodynamic parameters anesthesia was switched to intravenous Midazolam $(0.15 \mathrm{mg} / \mathrm{kg} / \mathrm{h})$ and Sufentanyl-forte $(3 \mathrm{mcg} / \mathrm{kg} / \mathrm{h})$, and the dogs were ventilated with room air. Under sterile conditions the thorax was opened through the right fifth intercostal space and the heart was exposed. After implantation of gold beads in the LV wall (see "X-ray marker implantation") the thorax was closed and pace leads were inserted transvenously through the jugular vein. A Medtronic capsure sp 4523 lead was positioned in the right atrial cavity and secured to its endocardial surface. A Medtronic $4057 \mathrm{M}$ unipolar screw-in ventricular lead was inserted into the apex of the RV. In 7 dogs ( $\mathrm{POH}$-pace, weighing $24.3 \pm 3.7 \mathrm{~kg}$ ) a pacemaker (Medtronic Synergist H7027, H7071, Elite II or Thera DR 7941) was implanted. In 4 dogs ( $\mathrm{POH}$-control, weighing $24.1 \pm 0.7 \mathrm{~kg}$ ) leads, but no pacemakers were implanted. For the assessment of the short-term effects of pacing in this group the leads were temporarily connected to an external pacemaker. The ECG was dexived from the limb leads. A dual tip micromanometer catheter (Sentron) was introduced into the left femoral artery to measure LV cavity and ascending aortic pressure (distal to the stenosis). Cardiac output was measured in triplicate by thermodilution during stopped ventilation, using a pulmonary artery catheter and a cardiac output computer (Edwards). Hemodynamic measurements were made under baseline conditions and 15 minutes after initiation of ventricular pacing. Subsequently, catheters were removed, flow in the vessels restored and the dogs were allowed to recover from 


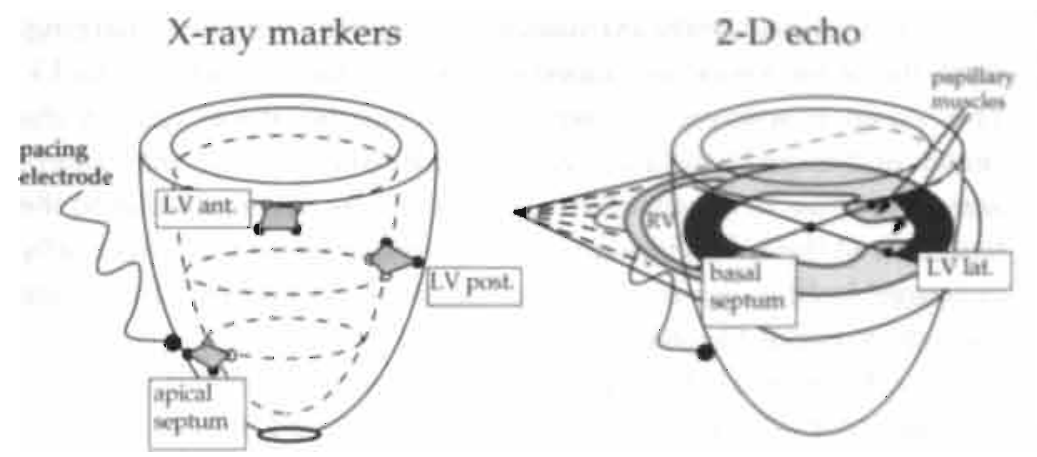

anesthesia and the surgical procedure, while the pacemaker was not functioning.

\section{X-ray marker implantation}

In $\mathrm{POH}$-control and $\mathrm{POH}$-pace animals radiopaque gold beads (diameter $1.5 \mathrm{~mm}$ ) were implanted in the ventricular wall to measure changes in regional myocardial wall volume. Quadruplets of markers were implanted at three different LV sites: A) 'apical septum': in the septum at the level of the RV apex, B) 'LV ant': in the basal anterior wall, near the division of the left anterior descending coronary artery and the left circumflex coronary artery and C) LV post': in the posterior wall, about $2 \mathrm{~cm}$ below the equator of the IV (Figure 7.1, left). Two of the 4 golden beads were implanted subendocardially and the other two underneath the epicardium (Figure 7.1). At each site the group of 4 markers formed the corners of a quadrangle with a circumferential distance of about $1.5 \mathrm{~cm}$ and a transmural distance of about $8 \mathrm{~mm}$. Care was taken that the markers were placed in a short axis plane. For dimensional calibra-
Figure 7.1 Schematic nqpesentation of X-ray marker and 2D echocantiographic moasurements, Lef: Quadruplets of gold bohis located at thre locations: the apioal scptum, (aidjacent to the $R V$ apex), the amterior $L V$ toall AV ant. near the division of the left anterior descrnding and cinciumflex corvnary artery) and the posterior LV wall (LV post, about $2 \mathrm{~cm}$ blow the equator of the LV), Right: 2D-echocandiogram, in a short-axis plane at the tip. of the papillary muscles. The dariker arcas indicate the wall sectors of the lateral LV wall (LV lat.) and the busal septum. Note that the apical septum nzgion is more distal and closely related to the pacing electrode whensis the basal septum ngion is located more proximal in the septum.

tion a golden ring, internal diameter $1 \mathrm{~cm}$, was secured to the tip of the LVapex.

\section{Protocol}

At age $9 \pm 1$ months in the $\mathrm{POH}$-pace group, ventricular pacing was started ( $t=0$, figure 7.2 ) approximately two weeks after the implantation procedure and continued for six months $(t=6$ months). The heart was stimulated at its own sinus rhythm by AV sequential pacing (DDD)-mode, uppei rate 175 beats/min). The AV stimulation interval was $25 \mathrm{~ms}$ to ensure complete ventricular capture. Froper pacemaker function was checked regularly. The $\mathrm{POH}$-control animals remained in sinus rhythm for six months.

Two-dimensional echocardiographic and X-ray images of the LV were made at $0,0.5,1,2,4$ and 6 months after onset of pacing in the POH-pace and at the same time intervals in the POH-control group. To this purpose, the dogs were slightly sedated by a mixture of acepromazine $(0.2 \mathrm{mg} / \mathrm{kg})$ and oxycodon $(1.2 \mathrm{mg} / \mathrm{kg})$. Parasternal short axis cross-sectionai echo images were taken, taking 


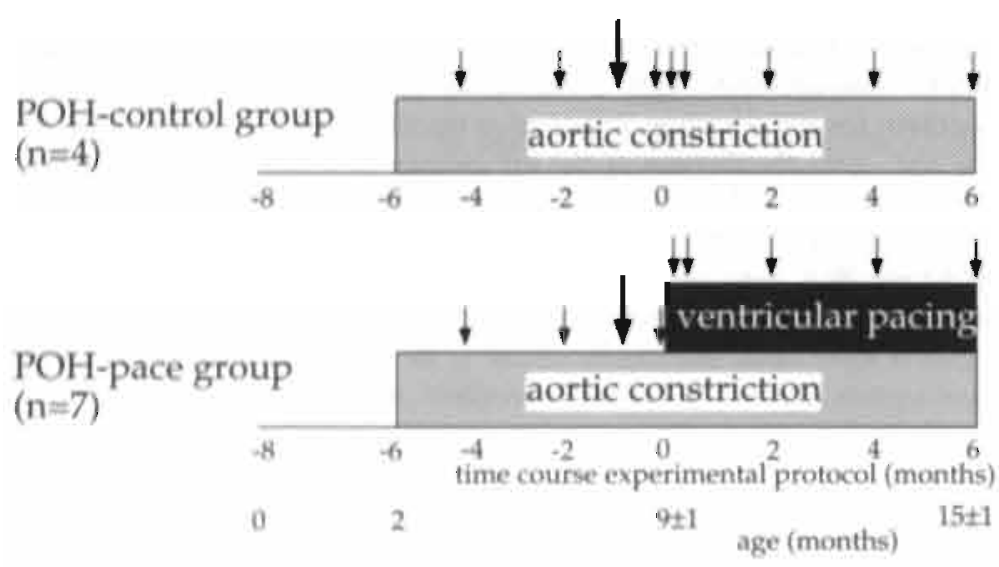

Figure 7.2 Diagram illustrating the experimental protocol. At 2 months of age ( $t=-6$ months) the ascending aorta was banded (shaded block). Before $9 \pm 1$ months of age (large black arrow) pacemakers and X-may markers were implanted. During the pacing period (between $9 \pm 1$ and $15 \pm 1$ months of age) dogs from the POH-pace group were paced (black block), whereas the dogs from the POH-sham group had normal sinus rhythm. At regular time intervals echo and X-ray measurements (small arrows) were performed. At implantation (two wecks before $t=0$ ) and terntination ( $t=6$ months) hemodymamics were determined during simus rhythm and during pacing.

care that the LV appeared as circular as possible and that the tip of the papillary muscles and the "LVant" group of implanted beads were visible Figure 7.1, right). These landmarks in the echo image enabled correlation of the site of tissue collection with the echographic image at termination. Subsequently $\mathrm{X}$-ray images were made with a Siemens Cardioskop U, equipped with a CCD camera
(756 $\times 485$ pixels, C4505, Hamamatsu Photonics) for video imaging. First, the camera was positioned in line with the long axis of the LV. This position was recognized by showing the apical ring in the center of the surrounding 3 groups of markers. Then, the camera was rotated by $90^{\circ}$ so that the camera axis was perpendicular to the long axis of the LV. In this image the markers per group practically appeared in line. For each animal the same camera positions were used throughout the study.

$\mathrm{X}$-ray and echocardiographic images were stored on superVHS tape for off line analysis. The tracing of lead II from the ECG was inscribed in the image by use of an analog video mixer.

\section{Terminal procedure}

After six months of pacing (POH-pace) or sinus thythm (POH-control) the final measurements were made, the dogs having reached the age of $15 \pm 1$ months. Anesthesia and catheter insertion were performed as at implantation (see above). Hemodynamic parameters were measured with functioning pacemaker and $15 \mathrm{~min}$ after the pacemaker had been switched off in the $\mathrm{POH}$-pace group and before and after 15 minutes of temporary pacing in the $\mathrm{POH}$ control group.

The heart was arrested in diastole by perfusion with icecold $\mathrm{CdCl}_{2}(0.1 \mathrm{M})$. The heart was quickly removed and the left and right ventricles were separated and weighed. For histological analysis samples (transmural slices of $15 \times 8 \times 4 \mathrm{~mm}$ ) were taken from the apical septum and from the LV lateral wall. Histological analysis consisted of determination of myocyte thickness, by use of the modified Azan staining, and collagen fraction, by use of the Sirius Red staining as described before. 


\section{Electrophysiologic and hemodynamic measurements}

Hemodynamic and ECG signals were digitized with 12 bits at $200 \mathrm{~Hz}$. Data were analyzed off-line. Using software developed in our laboratory the maximal rates of rise (LVdP/dtpos) and fall of $\mathrm{LV}$ pressure ( $\mathrm{LV} \mathrm{dP} / \mathrm{dtmin}$ ) were calculated as well as the duration of the QRS complex and the QT time of the ECG. The QT time was normalized for heart rate (QTc time) according to the formula of Bazett." The time constant of monoexponential LV pressure decline ( $\tau$ ) was calculated using $P(t)=P(0) \cdot \exp (-t / \tau)$, where $P(t)$ is $L V$ pressure at time $t$ and $P(0)$ is LV pressure at time LVdP/dtmin.

\section{Analysis of echo images}

Two-dimensional echocardiography was used to determine regional LV wall thickness and sector wall volume of a basal septum sector ("basal septum") and a lateral LV wall sector ("LV lat.", Figure 7.1, left panel). In an off line analysis, from digitized end-diastolic echo images wall thickness and volume were estimated as described in detail previously. In brief, epicardial and endocardial contours were marked manually in a digitized 2D-echo image of the LV short axis at end-diastole. Epicardial and endocardial contours were represented in polar coordinates with the center of the LV cavity as origin. Inner and outer radii were determined by fitting the epicardial and endocardial contourpoints to a closed curve with a limitation of the highest circular frequency to the fourth circular harmonic. The wall sector area of the lateral LV wall was defined by the endocardial and epicardial borders of the myocardial wall between the papillary muscles. The wall sector area of the basal septum was located in the short axis plane opposite to the LV free wall sector (Figure 7.1). Wall thickness of a sector was calculated as the difference between the epi and endocardial radius. Sector wall volume was calculated as the sector area multiplied by the mean LV radius, the latter being used as an estimate of general dilatation.' Cavity and wall volume of the entire LV were calculated from the $2 \mathrm{D}$ echo cross-sectional images and long axis dimensions by use of cylinder-ellipsoid model calculations. For calculation of the LV mass to body mass ratio the regression equation of the relation between postmortem LV weight $\left(W_{\text {LVwall,pm }}\right)$ and echocardiographically determined LV wall volume just before termination $\left(\mathrm{V}_{\mathrm{LV}}\right.$ wall, echo $)$ was determined. This relation could be described by the equation:

$$
\text { (1) } \mathrm{W}_{\mathrm{LV} \text { wall, } \mathrm{pm}}=-7.4+1.27 \times \mathrm{V}_{\mathrm{LV}} \text { wall, echo; } \mathrm{r}=0.93
$$

From this relation LV wall volume, determined at each time interval, was converted to LV wall mass.

\section{Analysis and assessment of X-ray images}

The $X$-ray measurements were used to estimate regional myocardial growth. In an off-line procedure for both mutually perpendicular viewing positions end-diastolic video images were selected and digitized, by use of a video frame grabber $(8$ bits grayscale, $768 \times 578$ pixels. DT3155, Data Translation, Inc., Marlboro; MA). ECG information was used to synchronize the pairs of images relative to the cardiac cycle. The images were considered to form a stereo pair and were used to reconstruct the 3D position of a marker. ${ }^{17}$ The digitized images were analyzed by use of $\mathrm{NIH}$ Image software (V 1.52). The image coordinates of each of the 12 markers were determined manually by means of the cross-hair tool. 
Using this method the root mean square error in marker position estimation is about $0.3 \mathrm{~mm}$.

For the estimation of sector wall volume sector wall area ( $A_{\text {sector, }}$ Xray) was calculated in each of the three locations with markers. To that purpose a plane was fitted to the 3-D marker positions. The projections of the marker positions to the plane were obtained and the area of the resulting quadrangle was calculated. Sector wall volume $\left(\mathrm{V}_{\text {sector, }} \mathrm{X}_{\text {ray }}\right)$ was estimated by

$$
V_{\text {sector, }} X_{\text {ray }}=\mathrm{D}_{\mathrm{a}-\mathrm{b}} \cdot \mathrm{A}_{\text {sector, }} \mathrm{X}_{\text {ray }}
$$

where $\mathrm{D}_{\mathrm{a}-\mathrm{b}}=$ the distance between the centers of gravity of the markers located at the RV apex and at the LV base. $D_{a-b}$ was used as an estimate of dimensional changes in the direction perpendicular to the short axis plane.

\section{Statistical analysis}

For each sector, growth, as determined by echocardiograpy or by X-ray marker analysis, was expressed relative to the state at $t=0$. Intra-individual changes in hemodynamics were evaluated by use of the Wilcoxon signed rank test and inter-individual changes by use of the Mann-Whitney-U test. The time course of global and local LV geometry was evaluated by analysis of variance (ANOVA)

Figure 7.3 Time course of body weight (A), LV wall mass (B), LV carvity volume (C) and LV wall mass/body weight ratio (D) in the POH-pace (closed circles) and POH-control group (closed squares). At time $=0$ pacemakers were turned on in the POH-pace gromp. Variables were normalized to their value at $t=0$.
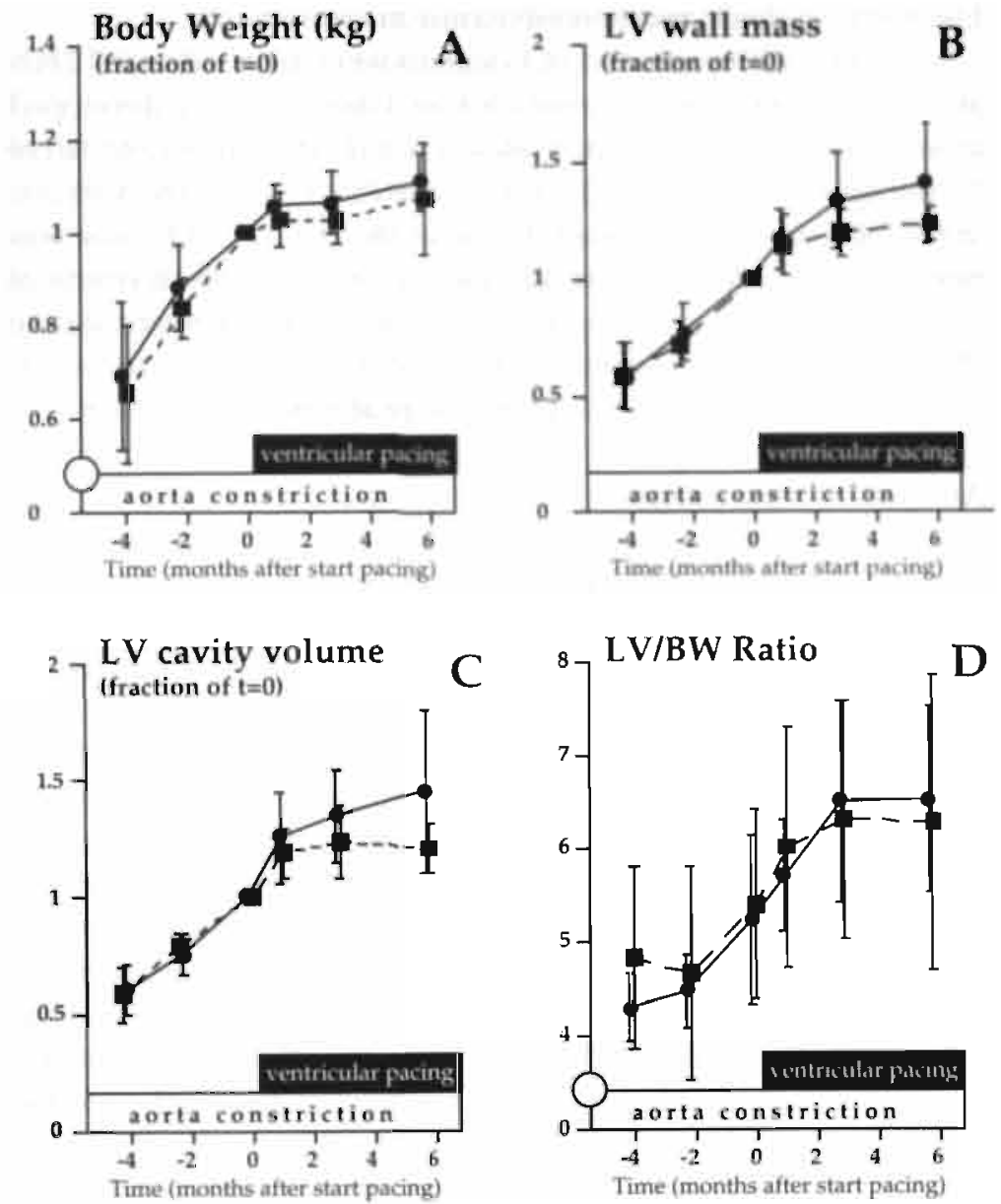
for repeated measurements. If significant differences were found, significant points were isolated by use of Bonferroni-Dunn correction. Data are presented as mean \pm SD. $\mathrm{P}<0.05$ was considered significant.

\section{RESULTS}

In all dogs in the $\mathrm{POH}$-pace group cardiac pacing was possible throughout the study period. None of the dogs in this study showed signs of cardiac failure.

\section{Changes in global cardiac geometry}

Figure 7.3 illustrates that body weight, LV wall mass, LV cavity volume and the LV wall mass/body weight ratio increased during the experimental period. In the $\mathrm{POH}$-pace and in the $\mathrm{POH}$-control group these parameters increased significantly during the months before $t=0$ (age $9 \pm 1$ months, the onset of pacing in the $\mathrm{POH}$-pace group) and during the first two months after $t=0$. The parameters stabilized towards the end of the experimental period. With respect to these parameters the $\mathrm{POH}$-control group and the $\mathrm{POH}$-pace group were not significantly different from each other at any time interval.

\section{Changes in regional cardiac geometry}

In the POH-control group sector wall volume in the five sectors studied (two echocardiographically, Figure $7.4 \mathrm{~A}$, and three by means of X-ray marker detection, Figure $7.4 \mathrm{C}$ ) increased on the average by $28 \%$ between $t=0$ and $t=6$ months. Increases in volume in individual wall sectors ranged from $27.0 \pm 9.2 \%$ in the apical septum to $29.8 \pm 5.8 \%$ in the lateral LV wall, differences between the various sectors being not statistically significant (Figure $7.4 \mathrm{~A}, \mathrm{C}$ ). Between $\mathrm{t}=0$ and $\mathrm{t}=6$ months thickness of the lateral LV wall and the basal septum, as assessed with echocardiography, increased significantly by $10.2 \pm 5.8 \%$ and $9.2 \pm 1.5 \%$, respectively (N.S. between these regions).

In the POH-pace group sector wall volume in the lateral LV wall and in the basal septum (measured by echocardiography) and in the anterior and posterior L.V wall (measured by means of X-ray marker detection) increased on the average by $33.3 \%$, ranging from $31.3 \pm 15.8$ in the basal septum to $35.2 \pm 17.0 \%$ in the posterior $\mathrm{LV}$ wall (Figure 7.4 B,D). In the apical septum, however, sector wall volume increased by only $17.2 \pm 21.5 \%$ between $t=0$ and $\mathrm{t}=6$ months, Figure 7.4D). This increase in apical septal wall volume was significantly smaller than the wall volume increases in the other four regions of the same 

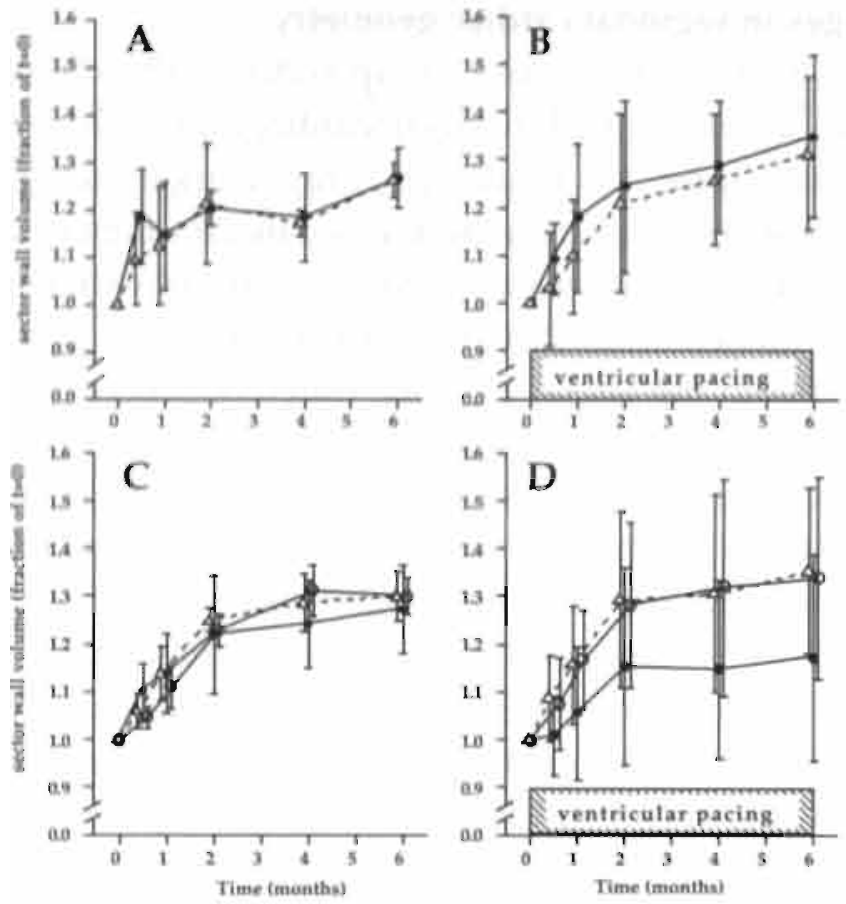

Figure 7.4 Time course of sector wall volume as determined by $2 \mathrm{D}$ echo $(A, B)$ and $X$-ray markers $(C, D)$ in POH-control $(A, C)$ and POH-pace groups $(B, D)$. Sector wall volume determined in $L V$ lat (closed cincles in $A, B$ ), basal septum (open triangles in $A, B$ ), $L V$ ant. (open triangles in $C, D$ ), LV post. (open circles in C,D) and apical septum (closed circles in C,D). For location of these regions see Figure 7.1. Note the suppression of laypertroplyy in the apical septum as compared to the four other regions in the POH-pace group (panel D). hearts. The increase in wall thickness in the lateral LV wall and the basal septum, as assessed with echocardiography, between $\mathrm{t}=0$ and $\mathrm{t}=6$ months, was $7.4 \pm 4.4 \%$ and $16.3 \pm 17.6 \%$, respectively, values not significantly different from those observed in the $\mathrm{POH}$-control group.

\section{Electrophysiology and hemodynamics}

Between $\mathrm{t}=0$ and $\mathrm{t}=6$ months the QRS duration during sinus rhythm increased significantly by $19 \pm 14.0$ $(\mathrm{p}<0.05)$ and $6.6 \pm 5.2 \mathrm{~ms}(\mathrm{p}<0.05)$ in the $\mathrm{POH}$-pace and $\mathrm{POH}-$ control group, respectively (N.S. between groups). In both groups acute $(15 \mathrm{~min})$ ventricular pacing more than doubled the QRS duration as compared with sinus rhythm ( $\mathrm{p}<0.05$, Table 7.1). In the POH-pace group the QRS duration further increased significantly by $24.0 \pm 17.8 \mathrm{~ms}$ during chronic pacing. In the $\mathrm{POH}$-control group QRS duration increased by $12.2 \pm 16.2 \mathrm{~ms}$ in the same period. Acute

Table 7.1 Hemodymamic effects of ventricular pacing during implantation and 6 months later during fermination. $S R=$ simus rhythm, HR = heart rate, PLVsys = systolic $L V$ pressure, $P L V e d=$ end-diastolic $L V$ pressure, $P A O$ sys $=$ systolic ascending (post stenotic) aortic pressure, SV = stroke volume. Mean values $\pm S D$ are presented. $p<0.05$ for the following comparisons: ventricular pacing compared with simus rhythm the same day ("); sinus thythm (t) and/or pacing $(\$)$ during implantation $(t=0)$ compared with those during termination ( $t=6$ months). 
Remodeling of Hypertrophying Myocardium by Pacing

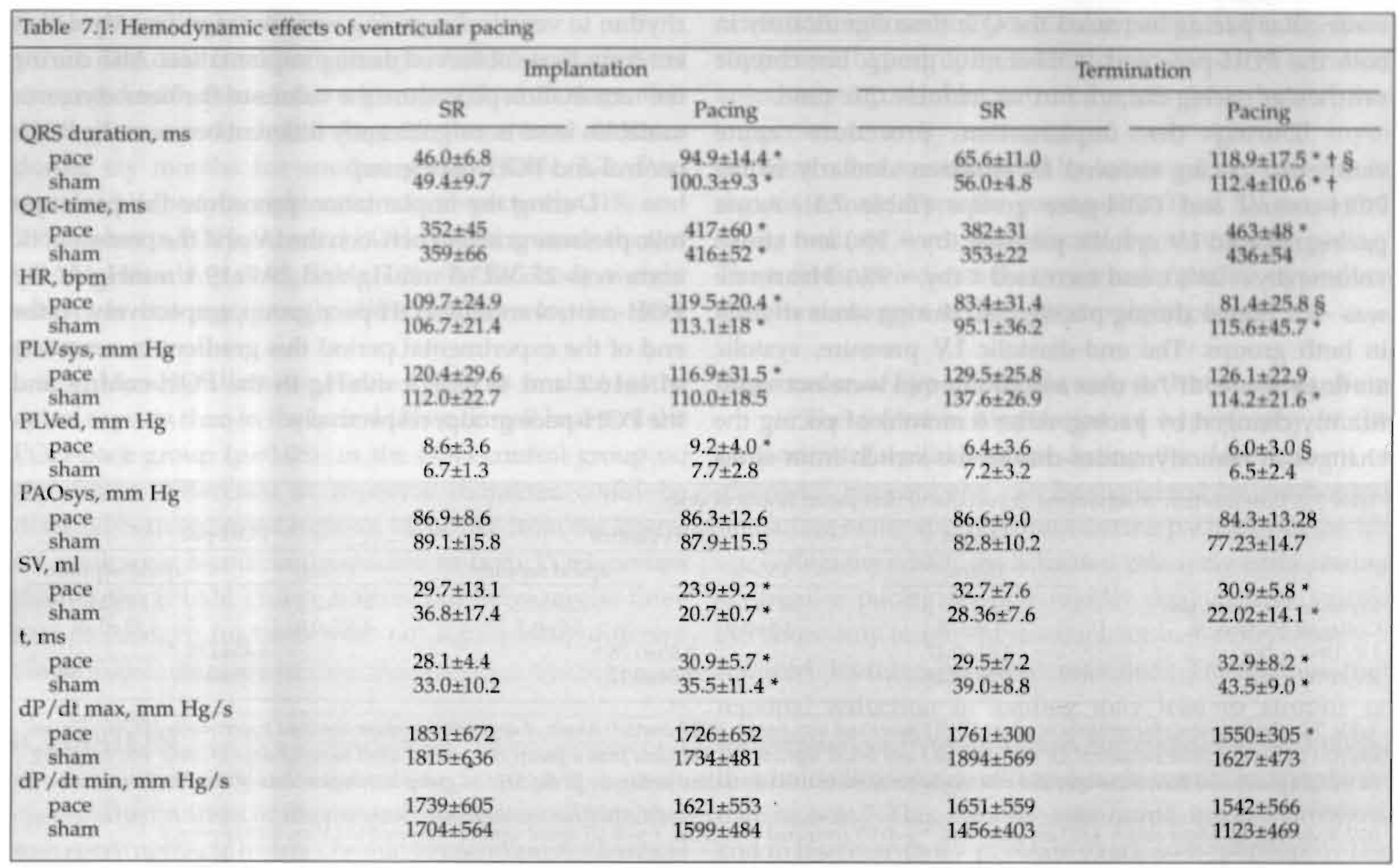


ventricular pacing increased the QTc time significantly in both the $\mathrm{POH}$-pace and $\mathrm{POH}$-control group, but chronic ventricular pacing did not further increase QTc time.

During the implantation procedure acute ventricular pacing reduced LV function similarly in the $\mathrm{POH}$-control and $\mathrm{POH}$-pace groups (Table 7.1). Acute pacing reduced LV systolic pressure (by $\sim 3 \%$ ) and stroke volume (by $\sim 28 \%$ ), and increased $\tau$ (by $\sim 9 \%$ ). Heart rate was $\sim 8 \%$ higher during pacing than during sinus rhythm in both groups. The end-diastolic LV pressure, systolic aortic pressure, $\mathrm{dP} / \mathrm{dt}$ max and $\mathrm{dP} / \mathrm{dt}$ min were not significantly changed by pacing. After 6 months of pacing the changes in hemodynamics due to the switch from sinus rhythm to ventricular pacing were not significantly different from those observed during implantation. Also during the termination procedure the values of the hemodynamic variables were not significantly different between the $\mathrm{POH}$ control and $\mathrm{POH}$-pace group.

During the implantation procedure the peak systolic pressure gradient between the LV and the post-stenotic aorta was $25.0 \pm 23.5 \mathrm{mmHg}$ and $29.7 \pm 19.4 \mathrm{mmHg}$ in the $\mathrm{POH}$-control and the $\mathrm{POH}$-pace group, respectively. At the end of the experimental period this gradient increased to $41.4 \pm 16.2$ and $44.6 \pm 23.4 \mathrm{mmHg}$ in the $\mathrm{POH}$-control and the $\mathrm{POH}$-pace group, respectively.

Table 7.2: Post mortem observations in paced and non-paced hearts of dogs

\begin{tabular}{|c|c|c|c|c|c|}
\hline & \multirow{2}{*}{$\begin{array}{l}\text { SHAM } \\
\text { LVant. }\end{array}$} & \multicolumn{2}{|c|}{ POH-control } & \multicolumn{2}{|c|}{ POH-pac } \\
\hline & & LVant. & apical septum & L.Vant. & apical septum \\
\hline Myocyte diameter, $\mu \mathrm{m}$ & $\begin{array}{r}22.3 \pm 1.9 \\
4.0 \pm 0.8\end{array}$ & $\begin{array}{c}25.1 \pm 2.4 * \\
4.1+0.9\end{array}$ & $\begin{array}{l}22.9 \pm 2.8 \\
3.7 \pm 0.2\end{array}$ & $\begin{array}{l}25.8 \pm 2.1^{*} \\
4.57 \pm 0.9\end{array}$ & $\begin{array}{l}21.7 \pm 2.6+ \\
4.32+0.39\end{array}$ \\
\hline LV/BW, g/kg & $4.91 \pm 0.47$ & \multirow{2}{*}{\multicolumn{2}{|c|}{$\begin{array}{l}5.85 \pm 0.78 \\
2.04 \pm 0.12\end{array}$}} & \multirow{2}{*}{\multicolumn{2}{|c|}{$\begin{array}{l}6.45 \pm 1.01 * \\
1.99 \pm 0.07\end{array}$}} \\
\hline $\mathrm{RV} / \mathrm{BW}, \mathrm{g} / \mathrm{kg}$ & $2.03 \pm 0.28$ & & & & \\
\hline
\end{tabular}

Table 7.2 Post mortem observations in paced (POH-pace) and non-paced (POH-control) hearts of dogs twith pressure overload hypertrophy (POH). Tissue samples were taken from the lateral LV wall ( $L V$ lat.) and apical septum. SHAM: data from a group of 5 healthy adult mongrel dogs of either sex, weighing $24.4 \pm 2.3 \mathrm{~kg}$, who had been sham operated and stayed for 6 months in our amimal facilities.' In the SHAM group histological datn of only the Loant were awailable.

BW: body weight. Mean values \pm SD are presentad. " $p<0.05$ compared to SHAM, $+p<0.05$ apical septum compared to LVlat. within groups. 


\section{Post mortem observations}

The data from both groups of $\mathrm{POH}$ animals were compared with those from a group of 5 adult dogs, that were sham operated and kept in the animal facilities during six months for another study ${ }^{3}$ (Table 7.2). Post mortem LV/body weight ratio was respectively $31 \%$ and $20 \%$ higher in $\mathrm{POH}$-pace and $\mathrm{POH}$-control animals than in SHAM animals $(p<0.05$, Table 7.2$)$; the RV/body weight ratio was not significantly different between the three groups.

Myocyte diameter (Table 7.2) was smaller in the apical septum than in the lateral LV wall in all hearts of the $\mathrm{POH}$-pace group $(\mathrm{p}<0.05)$. In the $\mathrm{POH}$-control group no significant difference in myocyte diameter could be detected between these regions. Myocytes from the lateral LV wall were significantly thicker in both $\mathrm{POH}$ groups than in the SHAM group. Within and between the three groups collagen fractions were not significantly different (Table 7.2).

\section{DISCUSSION}

The findings in the present study demonstrate that in hypertrophying hearts chronic asynchronous electrical activation, induced by ventricular pacing at the RV apex, suppresses hypertrophy selectively in the early-activated apical septum. Unlike in non-hypertrophic hearts' this suppression in hypertrophy of early-activated myocardium is not accompanied by increased hypertrophy in regions remote from the pacing site. Total LV mass and cavity volume were not affected by ventricular pacing. These findings indicate that ventricular pacing can induce structural changes in the hypertrophying heart.

\section{Suppression of hypertrophy in early-activated myocardium}

The selective suppression of hypertrophy in the early-activated apical septum during the development of global LV hypertrophy can be explained by mechanical unloading of the apical septum during pacing from the RV apex. Regions which are activated relatively early during ventricular pacing shorten rapidly during early systole but show only minor shortening later in systole, causing a reduced loading of these regions: ${ }^{1,2}$ The notion that regional reduction in loading may lead to atrophy or suppression of hypertrophy is supported by the finding that unloading of a papillary muscle results in atrophy of that muscle. ${ }^{19}$ This atrophy was found in normal hearts and in hearts with RV pressure overload hypertrophy. The 
finding that in hypertrophying hearts the LV wall grows less near the site of pacing than more remote from this site corroborates the findings in non-hypertrophic canine hearts' and in patients with left bundle branch block. ${ }^{20}$ Unlike in non-hypertrophic canine hearts ${ }^{3}$ wall volume is reduced in the early-activated regions in hypertrophying hearts (present study). This observation indicates that myocardial growth regulation is more susceptible to reduction in mechanical loading in hypertrophying than in non-hypertrophic hearts.

\section{Unchanged hypertrophy in late-activated myocardium}

Unlike in non-hypertrophic hearts ${ }^{3}$ in hypertrophying hearts ventricular pacing does not lead to increased hypertrophy in regions remote from the pacing site. Such an increase in hypertrophy might have been expected because during pacing workload is increased in late-activated regions due to the early systolic stretching followed by pronounced shortening later during systole. ${ }^{12}$ In normal hearts chronic ventricular pacing induces an increase in wall sector volume of $40 \%$ in the late-activated regions. It is unlikely that the lack of further hypertrophy, as found in the present study, is due to a maximum limit of hypertrophy. The increase in LV/body weight ratio of
$30 \%$ in our POH model is moderate as compared to the increases of $50-200 \%$ observed in other experimental studies. ${ }^{2122}$ The absence of increased hypertrophy in the late-activated regions may be caused by a reduced growth response of hypertrophic myocardium to mechanical stimulation as was shown in a study on isolated rat hearts. The reduced growth response in late-activated myocardium leads to less asymmetry in wall mass in paced hypertrophying than in paced non-hypertrophic hearts.

In the present study the dogs were paced from the RV apex, whereas in our previous study on nonhypertrophic hearts the dogs were paced from the LV free wall. It is unlikely that the less pronounced asymmetry of hypertrophy as observed in the present study is due to differences in the asynchrony of activation. Pacing from both sites more than doubles QRS duration. Moreover, MRI tagging studies showed that regional differences in fiber strain and fiber work are similar, though opposite, during RV apex and LV free wall pacing. ${ }^{23}$

\section{Possible clinical implications}

The finding that ventricular pacing reduces septal hypertrophy may explain the long-term effects of pacing 
therapy in HOCM patients. Ventricular pacing acutely reduces the ventriculo-aortic pressure gradient in these patients," an improvement that becomes even more pronounced during chronic ventricular pacing. ' These long-term improvements have been attributed to structural adaptations but in clinical studies the findings on the reduction of septal thickness are contradictory: a reduction in one study ${ }^{7}$ and no significant change in another." This discrepancy may be due to difficulties encountered in measuring or analyzing (apical) septal thickness on echocardiograms. In the present study the regional remodeling was demonstrated by means of $\mathrm{X}$-ray marker detection, a technique where exactly the same piece of tissue is followed over time.

Because the suppression of hypertrophy was most pronounced at the pacing site, LV function in patients with HOCM might be further improved by positioning the pacing lead closer to the basal septum. In this way reduction of the thickness of the basal septum, which is increased in HOCM patients, will be most effective. This may be at the cost of the acute reduction in LV-aortic pressure gradient, which was shown to be absent when pacing high in the septum. ${ }^{24}$ These investigators, however, aimed at minimal duration of the QRS complex and optimal acute hemodynamic responses, whereas local remodeling is anticipated to be optimal during chronic asynchronous activation.

The findings in the present study have to be extrapolated to HOCM patients with care. It is possible that the response of the myocardium of HOCM patients is different from that of hypertrophic canine myocardium due to pressure overload. Hypertrophy in HOCM patients is caused by a genetic disorder and (at least initially) not due to pressure overload. Besides, the thickened septum has an abnormal structure.

\section{Hemodynamic function}

The acute reduction of ventricular pump function during pacing has been well established. ${ }^{1.325}$ The findings in the present study demonstrate that in hypertrophic, as in non-hypertrophic hearts, ${ }^{3}$ LV function is not further affected by pacing for a period of 6 months.

\section{Experimental approach}

In the present study the pacing period started about 6-8 months after the banding procedure, when body weight reached a plateau. At that time LV hypertrophy was still developing. The $30 \%$ increase in LV mass found 
in the present study is in the lower range of that found by others ${ }^{21223}$ and was not associated with interstitial fibrosis, profound LV dysfunction or clinical signs of congestive heart failure. Therefore, the dogs in the present study had a moderate, compensated LV hypertrophy.

We used AV sequential pacing with a short AV interval ( $30 \mathrm{~ms}$ ) to ensure activation of the entire ventricle from the ectopic site. This setup enabled us to study cardiac function during sinus rhythm and ventricular pacing at implantation and termination. In patients changing the AV interval from 100 to $30 \mathrm{~ms}$ decreased cardiac output by $\sim 20 \% .{ }^{2,25}$ In a separate series of experiments in AV-blocked dogs we did not find a significant difference between pacing at AV intervals of 100 and $25 \mathrm{~ms}$ (Peschar and Prinzen, 1998, unpublished observations). More importantly, even if the short AV interval had impaired global LV function, it is very unlikely that it would have caused the regional remodeling as induced by pacing, the major finding of the present study.

\section{Conclusions}

The present study shows that chronic asynchronous activation of the hypertrophying left ventricle leads to remodeling of the LV wall. RV apex pacing selectively suppresses the development of hypertrophy in the apical septum. The absence of excessive hypertrophy in late-activated regions indicates that in hypertrophying myocardium myocardial growth has a reduced responsiveness for increased mechanical loading. The potential to induce such remodeling in hypertrophying myocardium may explain the long-term hemodynamic improvement in paced HOCM patients. Chronic RV apex pacing in hypertrophying hearts is not associated with further deterioration of LV function.

\section{REFERENCES}

1. Prinzer FW, Augustijn CH, Arts T, Allessie MA, Reneman RS. Redistribution of myocardial fiber strain and blood flow by asynchronous activation. Am J Physiol. 1990;259:H300-H308.

2. Delhaas T, Arts T, Prinzen FW, Reneman RS. Regional fibre stress-fibre strain area as an estimate of regional blood flow and oxygen demand in the canine heart. J Physiol (London). 1994;477,3;481-496.

3. Van Oosterhout MFM, Prinzen FW, Arts T, Schreuder JS, Vanagt WYR, Cleutjens JPM: Renernan RS. Asynchrounous electrical activation induces asymmetrical hypertrophy of the left ventricular wall. Circulation. 1998;98:588-595.

4. Schumkert $\mathrm{H}_{4}$ Weinberg EO, Bruckschlegel $\mathrm{G}$, Riegger AJ, Lorell BH. Alteration of growth response in established cardiac pressure overload hypertrophy in rats with aortic banding. I Clin Invest. 1995:96:2768-2774.

5. Kappenberger L, Linde C. Daubert C, McKenna W, Meisel E, Sadoul N, Chojnowska L. Guize I., Gras D, Jeannenaud X, et al. Pacing in hypertrophic obstructive cardiomyopathy. Eur. Heart ]. 1997;18:1249-1256.

6. Jeanrenaud X, Goy J-J. Kappenberger L. Effects of dual-chamber pacing in hypertrophic obstructive candiomyopathy. Lancet. 1992;339:1318-1823.

7. Fananapazir L. Epstein ND, Curiel RV, Panza JA, Tripodi D, McAreavey D. Long-term results of dual-chamber (DDD) pacing in obstructive hypertrophic cardiomyopathy. Evidence for progressive symptomatic and hemodynamic improvement and reduction of left ventricular hypertrophy. Circulation. 1994,90:2731-2742. 
8. Lister JW, Klotz DH, Jomain SL., Stuckey JH, Hoffman BF. Effect of pacemaker site on cardiac output and ventricular activation in doges with complete heart block. Am J Cardiol. 1964;14:494-503

9. Park RC, Little WC, O'Rourke RA. Effect of alteration of left ventricular activation sequence on the left ventricular end-systolic pressure-volume relation in closed: chest dogs. Circ Res. 1985:57:706-717.

10.Burkhoff D. Oikawa RY, Sagawa K. Influence of pacing site on canine left ventricular contraction. Am J PhysioL. 1986,251:H428-H435

11. Daggett WM. Bianco JA. Powell W]. Austen WG Relative contribution of the atrial systole-ventricular systole interval and of patterns of ventricular activation to ventricular function during electrical pacing of the dog heart. Circ Res. 1970;27:69 79.

12. Nakano K, Corin WJ, Spann IF, Biederman RWW, Denslow S, Carabello BA Abnormal subendocardial blood flow in pressure overload hypertrophy is associated with pacing-induced subendocardial dysfunction. Circ Res. $1989.65 \cdot 1555-1564$

13.Omens JH, Covell JW. Transmural distribution of myocardial tissue growth by volume-overload hypertrophy in the dog. Circulation. 1991;84:1235-1245.

14. Bazett HC. An analysis of the time relations of electrocardiograms. Heart $1920: 7 \cdot 353-368$

15.Gaynor JW, Feneley MP, Gạll SA, Maier GW, Kisslo JA, Davis JW, Rankin JS, Glower ir. DD. Measurement of left ventricular volume in normal and volumeoverloaded canine hearts. Am I physiol. 1994:266:H329-H340.

16. Wyatt HI. Heng MK. Meerbaum S, Hestenes ID, Cobo !M, Davidson RM, Corday E. Cross-selectional echocardiography $\mathrm{L}$. analysis of mathematic models for quantifying mass of the left ventricle in dogs. Circ. 1979,60:1104-1113.

17Muiitiens AMM, Roos IMA, Van Oosterhout MFM, Prinzen FW, Egmont-Petersen M. Arts T, Reneman RS. Measuring regional hypertrophy by radiopaque myocardial markers viewed in 3-D with a single X-ray camera. In: Computers in cardiology 1997. IEFE computer society Press, Washington,. 565- 568.

18. Muititiens AMM Roos IMA. Arts T, Hasman A Reneman RS. Simultaneous estimation of stereo correspondence and camera geometry from marker tracks. In: Computers in cardiology 1995. IEEE Computer Society Press, Washington. 577-580.

19. Cooper IV G, Kent RL., Uboh CE. Thompson EW, Marino TA. Hemodynamic versus adrenergic control of cat right ventricular hypertrophy. J Clin Invest. 1985;75:1403-1414.

20.Prinzen FW, Cheriex EC, Delhaas T, Van Oosterhout MFM, Arts T. Wellens HJJ. Reneman RS. Asymmetric thickness of the left ventricular wall resulting from asynchronous electric activation: A study in dogs with ventricular pacing and in patients with left bundle branch block. Am Heart J. 1995:130:1045-1053.

21. Tagawa H, Koide M, Sato H, Zile MR, Carabello BA, Cooper IV G. Cytoskeletal role in the transition from compensated to decompensated hypertrophy during adult canine left ventricular pressure overloading. Circ Res. 1998:-82.751-761.

22. Hittinger L. Mirsky L, Shen Y-T, Patrick TA, Bishop SP, Vatner SF. Hemodynamic mechanisms responsible for reduced subendocardial coronary reserve in dogs with severe left ventricular hypertrophy. Circulation. 1995-92:978-986.
23. Prinzen FW. Hunter WC, Wyman 8I. McVeigh. ER. Mapping of regional myocandial strain and work during ventricular pacing: experimental study using MRI tagging. JACC, in press. 1999 ,

24.Gadler E. Linde C, Juhlin-Dannfeldt A, Ribeiro A, Kydên L. Influence of tight ventricular pacing site on left ventricular outflowtract obstruction in patients with hypertrophic obsinuctive cardiomyopathy. I Am Coll Cardiol. 1906:27:1219-1224.

25 Rosenqvist M, Bergfeldt L. Haga X. Ryden I. Ryden L. Owall A. The effect of ventricular activation sequence on cardiac performanoe during pacing. Pace $199619-1279-1286$

26. Urabe Y, Mann DL, Kent RL. Nakano K, Tomanek RI, Carabello BA, Cooper IV G. Cellular and ventricular contractile dysfunction in experimental canine mitral regurgitation. Circ Res. 1992:70:131-147.

27 Samet B. Castillo P. Bernstein WH. Hemodynamic consequences of sequential atrioventricular pacing. Am I Candiol. 1968,21:207-212

28. Faerestrand S, Oie B, Ohm O- . Noninvasive assessment by doppler and M-mode echocardiography of hemodynamic responses to temporary pacing and to ventriculoatrial conduction, PÁCE. 1987:10:871-885. 


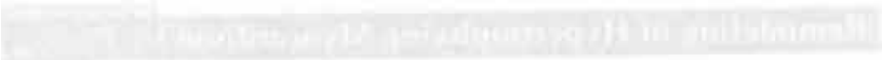




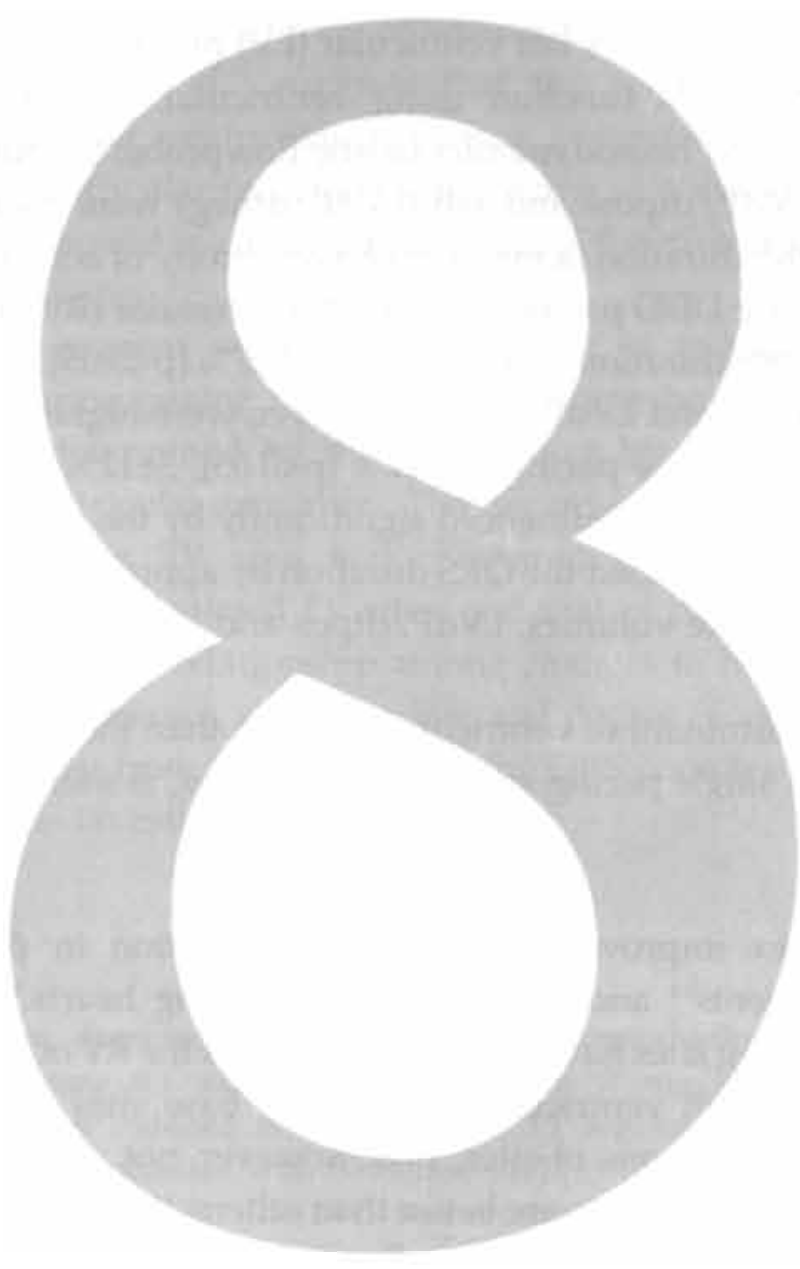

\section{OPTIMIZATION OF VENTRICULAR FUNCTION BY IMPROVING THE ACTIVATION SEQUENCE DURING VENTRICULAR PACING}

F.W. Prinzen, M.F.M. van Oosterhout, W.Y.R. Vanagt, C. Storm*, R.S. Reneman

Department of Physiology, Cardiovascular Research Institute Maastricht (CARIM) and Heart Center*, Rotterdam, The Netherlands 
Background - Abnormal electrical activation during ventricular pacing reduces left ventricular (LV) pump function.

Methods and Results - Two strategies were compared to optimize LV function using ventricular pacing: minimal asynchrony and optimal sequence of electrical activation. ECG and hemodynamics (aortic flowprobe, thermodilution cardiac output, LV pressure and its maximal rates of rise (LVdP/dtpos) and fall (LVdP/dtneg) were measured in anesthetized open-chest dogs ( $n=7$ ) with healthy hearts. The QRS duration (a measure of asynchrony of activation) was $47 \pm 5$ ms during sinus rhythm and increased to $110 \pm 12 \mathrm{~ms}$ during DDD pacing at the right ventricular (RV) apex with short AV-interval. During pacing at the LV apex and LV base, QRS duration was $8 \pm 7 \%$ and $15 \pm 7 \%$ [p<0.05] longer than during RV apex pacing, respectively. Stroke volumes, LVdP/dtpos and LVdP/dtneg, however, were higher during LV apex $(15 \pm 16 \%, 10 \pm 12 \%[\mathrm{p}<0.05]$ and $15 \pm 10 \%$, respectively) and LV base pacing $(11 \pm 12 \%$ [p<0.05], $3 \pm 12 \%$ and $3 \pm 11 \%$, respectively) than during RV apex pacing. Systolic LV pressure was not influenced significantly by the site of pacing. Biventricular pacing (RV apex together with one or two LV sites) decreased the QRS duration by approximately $20 \%$ as compared with RV apex pacing, however, it did not improve stroke volumes, LVdP/dtpos and LVdP/dtneg beyond those during pacing at the LV apex alone.

Conclusions - The sequence of electrical activation is a stronger determinant of ventricular function than the synchrony of activation. For optimal LV function the selection of an optimal single pacing site, like the LV apex, is more important than pacing from multiple sites.

$\mathbf{V}$ entricular pacing is known to reduce ventricular function in terms of contractility, relaxation and stroke volume. ${ }^{\text {is }}$ Although the timing of ventricular activation with respect to atrial activation is an important determinant of this reduced function, ${ }^{34}$ the site of pacing has been recognized to influence pump function. ${ }^{1}$ Recent interest has developed in pacing sites other than the RV apex improving hemodynamic function in pacemaker patients $^{-s}$ and in patients with failing hearts. ${ }^{2.12}$ Several pacing sites have been tested, such as the RV outflow tract, the high ventricular septum, LV base sites and various combinations of sites. It is, however, not yet understood why some sites are better than others. While the results of several studies suggest that ventricular function can be 
optimized by better synchrony of ventricular activation, a study of Little et al. ${ }^{13}$ suggests that the sequence of activation of the ventricular wall is a potential determinant of ventricular function, since pacing at a number of LV sites resulted in consistently better LV function than pacing at RV sites.

The present study was designed to evaluate whether, during pacing of the normal canine heart, LV function is determined by the sequence or by the synchrony of ventricular activation. The hemodynamic effect of pacing at the RV apex was compared with that of pacing at various isolated LV sites, and that of pacing at multiple sites. The relationship among changes in hemodynamic performance, site of pacing, and degree of asynchrony derived from the duration of the QRS complex of the ECG was investigated.

\section{METHODS}

Seven dogs were premedicated with acepromazine 0.2 $\mathrm{mg} / \mathrm{kg}$, atropine $0.1 \mathrm{mg} / \mathrm{kg}$ and oxycodone $2 \mathrm{mg} / \mathrm{kg} \mathrm{IM}$. Anesthesia was induced with thiopental $15 \mathrm{mg} / \mathrm{kg}$ IV and maintained by ventilation with halothane $(0.75$ to $1.5 \%)$ in a $1: 2$ mixture of $\mathrm{O}_{2}$ and $\mathrm{N}_{2} \mathrm{O}$. The ECG was recorded from the limb leads. LV pressure was measured with a double-tipped cathetertip manometer (Sentron), introduced through a carotid artery. After opening the chest, an aortic flowprobe (Transonic) was positioned around the ascending aortic to measure instantaneous aortic flow. Absolute values of aortic flow were calibrated in each experiment with use of thermodilution (Baxter catheter and cardiac output computer). Pacing leads (Medtronic, temporary myocardial pacing leads, type 6500) were attached to the right atrium, the endocardium of the RV apex, and the epicardium of the LV apex and LV base. The leads were connected with a Medtronic Synergist II pacemaker. For multisite pacing the various ventricular leads were connected to each other with a metal clamp. In all cases the pacing electrodies served as a cathode. The pacemaker case, positioned subcutaneously, served as an anode.

After completion of the preparation and a stabilization period, measurements were performed during pacing from the various sites alone and during pacing at the $\mathrm{RV}$ apex in combination with one or two LV sites. Pacing was performed in the DDD mode with short $(25-30 \mathrm{~ms}) \mathrm{AV}$ intervals, to assure that the entire ventricle was activated from the ectopic site(s).

Hemodynamic performance was assessed from LV pressure, cardiac output (thermodilution, aortic flow probe) and derived parameters. Pressure, aortic flow and ECG signals were digitized at $200 \mathrm{~Hz}$ and stored on disk for off-line analysis, Stroke volume was calculated from the time integral of aortic flow velocity during the ejection phase, as measured by the flow probe. The maximal first positive ( $\mathrm{LVdP} / \mathrm{dtpos}$ ) and negative derivative of $\mathrm{LV}$ pressure (LVdP/dtneg) were calculated from the digitized signals using MATLAB software and were used as measures for contractility and relaxation, respectively. An ECG recorded from the 
limb leads was used to obtain QRS duration. QRS duration was used as measure for the synchrony of ventricular activation. Measurements were performed over 5-10 heart beats, 3 min after the onset of a particular pacing mode. The various pacing sites were paced in random order. After studying two or three different pacing modes, measurements were obtained again during sinus rhythm to

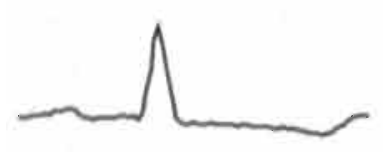

sinus rhythm

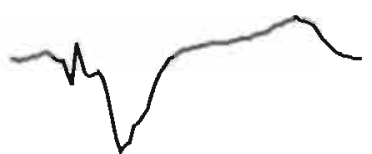

RV apex + LV base pacing

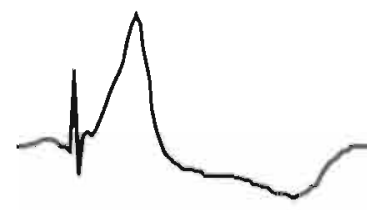

RV apex pacing

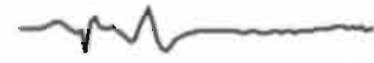

LV base pacing

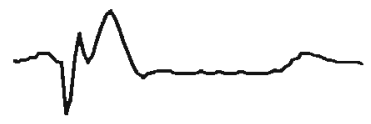

RV apex + LV apex pacing

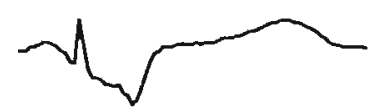

$\mathrm{LV}$ apex pacing $\overline{100 \mathrm{~ms}}$

Figure 8.1 Tracings of ECG (lead II) in a dog during simus ritythm and during pacing at the various left-and rightoentricular sites. verify the stability of the preparation.

The statistical significance of the difference of variables during pacing at the various sites was evaluated using the Wilcoxon's rank sum test. A P value $<0.05$ was considered significant.

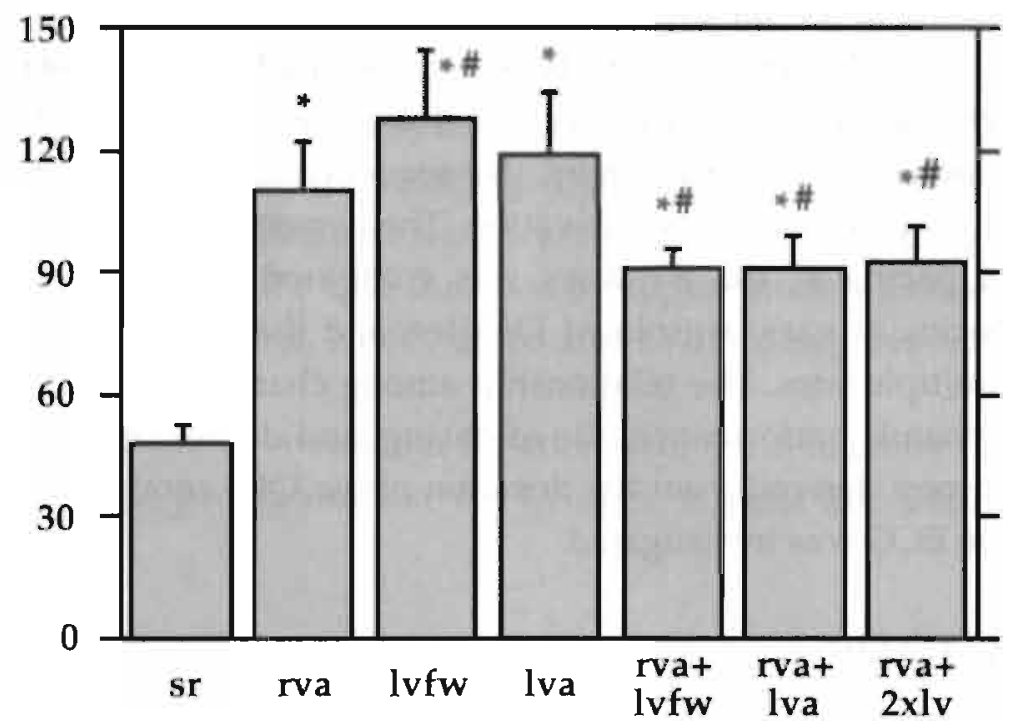

Figure 8.2 QRS duration (ms) during sinus rhythm (SR), RV apex (roa), LV base (Ivb), LV apex (loa) pacing and during pacing at the RV apex in combination with one or two LV sites. Pacing was DDD pacing with $A V$ interoal 25 -30 ms. * $P<0.05$ vs $S R$; \# $P<0.05$ vs RVA pacing. 


\section{RESULTS}

Heart rate was $108 \pm 21$ beats/min during sinus rhythm and did not change significantly throughout the experimental protocol. Systolic and end-diastolic LV

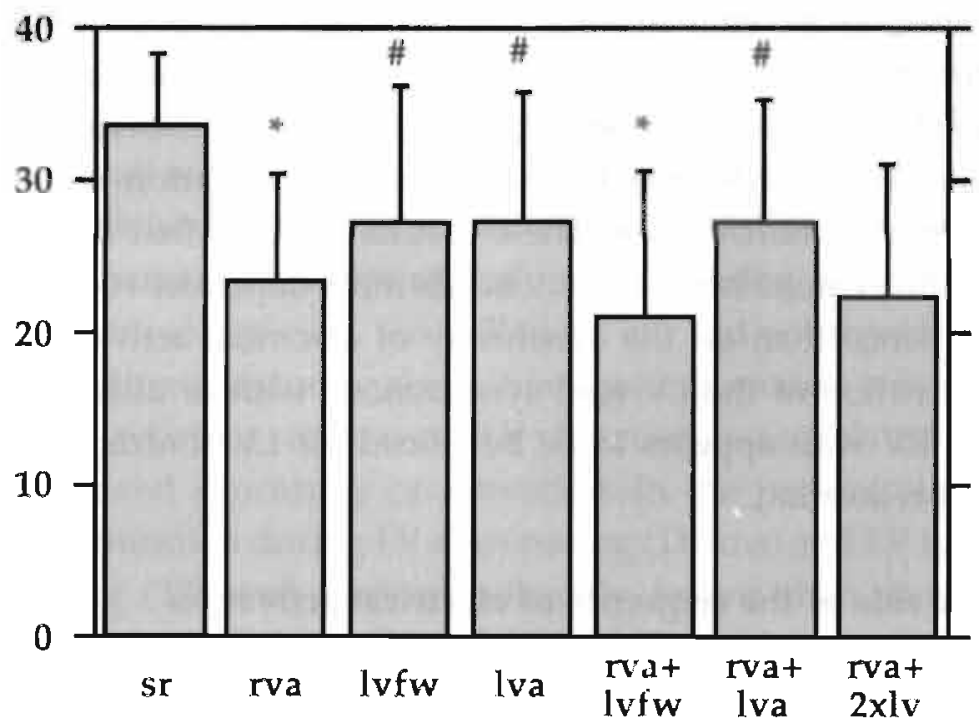

Figure 8.3 Stroke volume $(m l)$ during simus rhythm (SR), RV apex (roa), LV base (lvb), LV apex (lva) pacing and during pacing at the RV apex in combination with one or two LV sites. Pacing was DDD pacing with AV interval $25-30$ ms. * $P<0.05$ vs $S R ; \# P<0.05$ vs RVA pacing. pressure were $102 \pm 7 \mathrm{mmHg}$ and $5 \pm 4 \mathrm{mmHg}$, respectively, during sinus rhythm and were not significantly changed by ventricular pacing. Figure 8.1 presents ECG tracings (lead II) obtained during pacing at the various sites and combination of sites, As compared with sinus rhythm all modes of ventricular pacing increased QRS duration significantly. RV apex pacing increased QRS duration less than LV apex pacing and LV base pacing (Figure 8.2). Biventricular pacing (RV apex in combination with LV apex, LV base or both LV sites) shortened QRS duration by approximately $20 \%$ as compared with RV apex pacing alone (Figure 8.2).

During RV apex pacing stroke volume was approximately $30 \%$ lower than during sinus rhythm and $15 \%$ lower than during LV apex and LV base pacing (Figure 8.3). There was no significant difference in stroke volume between sinus rhythm and LV apex or LV base pacing. Biventricular pacing resulted in stroke volume values higher than during RV apex but similar to those during $\mathrm{LV}$ apex (Figure 8.3). The changes in LVdP/dtpos and $\mathrm{LVdP} / \mathrm{d}$ tneg induced by pacing at the various sites were similar to those for stroke volume (Figure 8.4). 


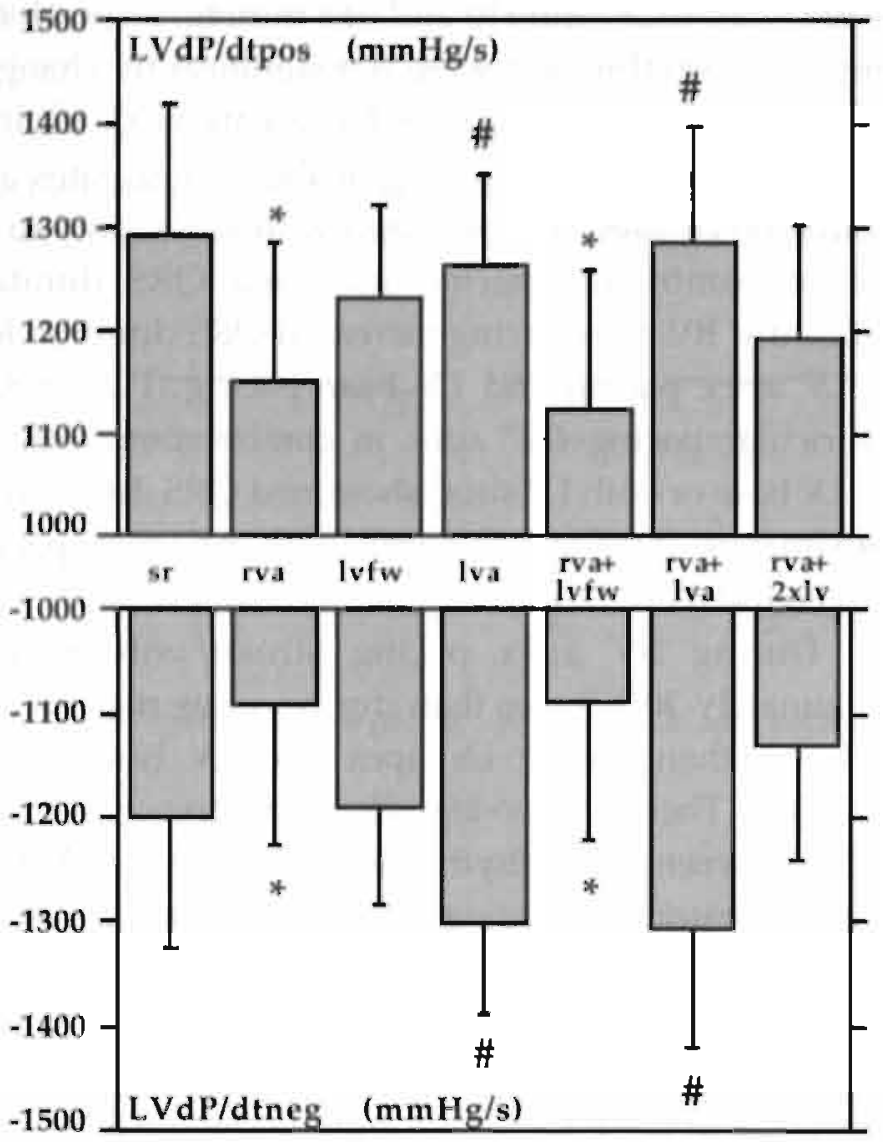

Figure 8.4 LVdP/dtpos and LVdP/Atneg during single or multisite pacing. Abbreviations as in Figure 8.2.

\section{DISCUSSION}

The present study, performed in the normal canine heart, demonstrates that pacing with short AV interval at a single LV site results in better cardiac function than pacing at the conventional site (RV apex), although QRS duration was longer during, LV pacing. Ventricular function was comparable during LV apex and biventricular pacing, despite a 30\% longer QRS duration when pacing at the LV apex. These results indicate that in the healthy canine heart LV function is more dependent on the sequence than on the synchrony of electrical activation. Activation of the LV wall synchronous with or ahead of the RV wall appears to be beneficial for LV contractility and relaxation.

\section{The role of the sequence of electrical activation}

The deminished LV function during pacing at the RV apex has been known for decades. ${ }^{135,13}$ Recent studies in animals and humans have shown improved hemodynamic function when pacing from alternative sites, such as the high ventricular septum ${ }^{7 s}$ and the RV outflow tract. ${ }^{0.12}$ Since pacing at these sites leads to narrower QRS complexes, optimal synchronization of activation appeared 
to be important to improve ventricular function. The present study shows that RV apex pacing causes poorer LV function than pacing at the LV wall, despite a shorter QRS duration during RV apex pacing. These findings appear in agreement with observations of Blanc et al. ${ }^{n}$ in patients with congestive heart failure and abnormally long intraventricular impulse conduction (QRS duration $171 \pm 31 \mathrm{~ms}$ ). In these patients pacing at the endocardial LV free wall was superior to pacing at the RV apex. Moreover, in the study of Blanc et al. " as well as in the present study, LV function was comparable during LV pacing and during biventricular pacing. Since Blanc et al." did not report the QRS duration during the various pacing modes, it is not clear whether improved LV function was related to improved synchrony of activation. In the present study QRS duration during LV apex pacing ( $119 \mathrm{~ms}$ ) and LV base pacing (128 ms) were significantly longer than during. biventricular pacing $(91 \mathrm{~ms})$, indicating that improved LV function as compared with RV apex pacing does not depend strictly on more synchronous activation. The superiority of LV pacing over RV apex pacing in the normal dog heart: (present study) and in the failing human heart ${ }^{11}$ may indicate that LV pacing does not simply correct an abnormality of the diseased heart, such as left bundle branch block, but may be due to an intrinsic property of the heart .

A classical theory on the cause of reduced ventricular function during ventricular pacing is the "loss of effective muscle mass". This theory states that during pacing the ventricle looses part of its effective muscle mass due to the reduced function of the early activated regions. Studies of regional mechanics during pacing studies have shown that early activated regions were indeed hypofunctional, but also that late activated regions were hyperfunctional. ${ }^{4.15}$ The degree of reduction of ventricular function could then depend on the degree of asynchrony, but also on the relative size of early and late activated regions. Preliminary results on mapping of mechanical activation of the canine left ventricle using the MRI tagging technique indicate that during LV base pacing impulse conduction spreads gradually, whereas RV apex pacing was associated with a biphasic spread of activation and a relatively large (septal) early activated region, ${ }^{\text {th }}$ which could explain the relatively large loss of function during RV apex pacing.

An alternative explanation could be that early LV activation is preferable because of optimal interventricular coupling. This is supported by studies from Little et al.," 
who compared RV and LV pressure development and septal motion during pacing from various LV and RV sites. These investigators reported better cardiac function when pacing from LV sites. Poorer LV function during pacing from RV sites was associated with paradoxical septal motion due to an earlier rise in RV than LV cavity pressure.

Interestingly, pacing at the various sites had similar effects on measures of contractility (stroke volume, $\mathrm{LVdP} / \mathrm{dtpos}$ ) and relaxation ( $\mathrm{LVdP} / \mathrm{dtneg}$ ). This link between contractility and relaxation could be explained by changes in stroke volume. A larger stroke volume, induced by a higher contractility, would reduce endsystolic load, known to accelerate relaxation. ${ }^{17}$ However, according to the "triple control of relaxation" theory of Brutsaert and Sys, ${ }^{17}$ smaller nonuniformity, presumably associated with minimal asynchrony, would be expected to lead to faster relaxation. The observation that $\mathrm{LVdP} /$ dtneg values are also high during LV pacing, despite relatively wide QRS complexes, suggests that specific contraction and relaxation patterns, rather than asynchrony itself, determine relaxation.

\section{Possible practical implications}

The present study was performed in healthy, anesthetized open-chest dogs. These conditions differ in various respects from the situation in cardiac patients with pacemakers. Therefore, extrapolation of the findings in the present study to patients should be done with care. Nevertheless, its results may have some relevance for the use of pacing in patients. While it was known for a long time that the conventional RV apex pacing site was not optimal for hemodynamic function, the present study indicates that optimal ventricular function can be obtained by pacing at a single LV pacing site, particularly the LV apex. Pacing at multiple sites did not seem advantageous, which facilitates practical application. The beneficial hemodynamic results of LV pacing in normal canine hearts as well as in failing human hearts ${ }^{11}$ indicates that patients with conventional pacemaker indications as well as patients with heart failure may benefit from left ventricular pacing.

Epicardial LV pacing sites, as used in the present study, are also accessible via the coronary veins for longterm pacing. ${ }^{18}$ The LV apex, the site resulting in the best hemodynamic performance, may, however, be difficult to reach, since it would require advancement of the leads to the most apical parts of the veins. Endocardial LV pacing, as used in short-term studies by Blanc et al,," need more 
investigation because of the risk of thromboembolic complications.

\section{Conclusions}

Pacing the LV apex results in better ventricular function than pacing at the RV apex and was comparable to biventricular pacing, despite a longer QRS duration when pacing from the LV apex. Therefore, the sequence of electrical activation of the ventricles may be a more important determinant of ventricular function than the synchrony of activation.

\section{REFERENCES}

1. Lister JW, Klotz DH, fomain SL, Stuckey JH, Hoffman BF. Effect of pacemaker site on cardiac output and ventricular activation in dogs with complete heart block. Am / Cardiol 1964:14:494-503.

2. Heyndrickx GR, Vantrimpont P., Rousseau MF, Pouleur H. Effects of asynchrony on myocardial relaxation at: rest and during exercise in conscious dogs. Am Physiol 1988:254:H817-1:1823

3. Daggett WM, Bianco JA. Powell WJ, Austen WG. Relative contribution of the atrial systole-ventricular systole interval and of patterns of ventricular activation to ventricular function during electrical pacing of the dog heart. Circ Res $1970: 27: 69-79$

4. Samet P. Castillo P, Bernstein WH. Hemodynamic consequences of sequential atrioventricular pacing. Am J Cardiol 1968;21:207-212.

5 Burkhoff D, Oikawa RY, Sagawa $K$. Influence of pacing site on left ventricular contraction Am I Physiol 1986-251:H428-H435.

6. Iberer F. Cardiac pacing via the right ventricular outflowtract. A human open chest study. Acta Chirurg Austriaca 1990:22:3-14.

7. Rosenqvist M. Bengfeldt L. Haga Y, Rydén L. Owall A. The effect of ventricular activation sequence on cardiac performance during pacing. PACE 1996;19:1279. 1287.
8. Karpawich PB, Justice CD, Chang C-H, Gause CY, Kuhns L.R Septal ventricular pacing in the immature canine heart: $\mathrm{A}$ new perspective. Am Heart I $1991121827-833$

9. Buckingham TA. Candinas R. Schlapfer I, et al. Acute hemodynamic effects of atrioventricular pacing at different sites in the right ventricle individually and simultaneously. PACE 1997:20:909-915.

10. Cazeau S, Ritter P. Lazarus A, et al. Multisite pacing for end-atage heart failure sarly experience. PACE 1996; 19-1748-1757.

11. Blanc II, Etienne Y, Gilard M, et al. Evaluation of different ventricular pacing siter in patients with severe heart failure: results of an acute hemodynamic study Circulation 1997,96,3273-327\%

12. De Cock CC, Meijer A, Kamp O, Visser CA. Hemodynamic benefits of right ventricular outflow tract pacing comparison with right ventricular apex pacing PACE 1998:21:536-541

13. Little WC, Reeves RC. Arciniegas I, Katholi RF, Rogen HW. Mechanism of abnormal interventricular septal motion during delayed left ventricular activation. Circ Res 1982,65:1486-1490.

14. Prinzen FW, Augustin CH, Arts T, Allessie MA, Reneman RS. Redistribution of myocardial fiber strain and blood flow by asynchronous activation. Am / Physio 1990,259-H300-H308.

15. Prinzen FW, Hunter WC, McVeigh ER. Mapping of contractile inhomogeneity during ectopic ventricular stimulation using MRI tagging. Circulation 1996,94:I. 387.

16. Prinzer FW, Wyman BT, Hunter WC, McVeigh ER. Non-invasive mapping of mechanical activation and work in the left ventricular wall during asynchmonous electrical activation using MRI tagging. PACE 1998: Cardiostim 98, session $193 / \mathrm{PW}$

17. Brutsaert DL. Sys SU. Relaxation and diastole of the heart. Physiol Rev 1989:69:1228-1301

18. Daubert JC, Ritter $\mathrm{P}$, Le Breton $\mathrm{H}$, et al. Permanent left ventricular pacing with transvenous leads inserted into the coronary veins. PACE 1998;21:239-245. 


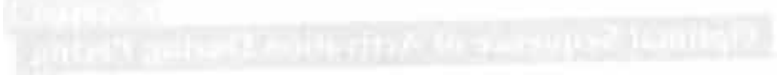




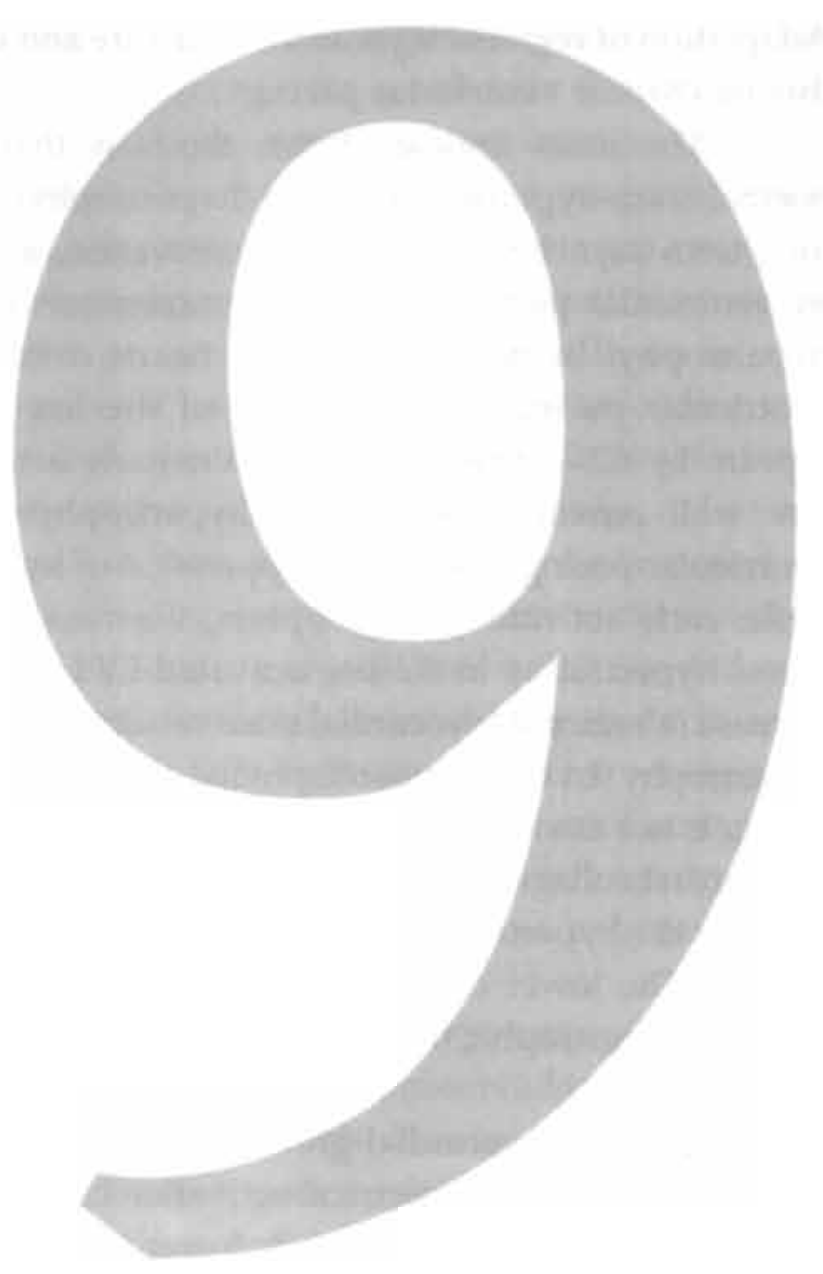

GENERAL DISCUSSION 
A. Imost 400.000 pacemakers are implanted worldwide each year.' Surprisingly, the impact of long term asynchronous activation on myocardial structure and function are not well-known. The aim of this thesis was to study the adaptation of the myocardium to asynchronous electrical activation, as induced by chronic ventricular pacing in long term animal experiments. The major question addressed in this thesis is whether a long lasting condition of local differences in workload between various regions in the left ventricle leads to locally different degrees of myocardial growth (hypertrophy). Nonuniform distribution of workload was achieved by pacing induced asynchronous electrical activation. In this respect it was investigated whether structural changes, induced by pacing, are different between normal and hypertrophying hearts. Furthermore, it was investigated whether local differences in the degree of hypertrophy are associated with local differences in the activity of metabolic enzymes and myocardial blood flow. To the latter end the fluorescent microsphere method to measure myocardial blood flow was improved and validated for the use in chronic experiments. The effect of chronic pacing on global pump function was investigated as well. Finally, it was studied whether in pacing hemodynamic function can be optimized by pacing from different site(s).
Adaptation of regional myocardial structure and geometry during chronic ventricular pacing

The main finding of this thesis is that in both normal (non-hypertrophied) and hypertrophying hearts long-term asynchronous electrical activation, as induced by ventricular pacing, leads to asymmetrical ventricular hypertrophy. In non-hypertrophic hearts, 6 months of ventricular pacing increases mass of the late activated septum by $40 \%$, whereas mass of the early activated LV free wall remains constant. In hypertrophying hearts ventricular pacing results in a suppression of hypertrophy in the early activated apical septum, whereas there is no excess hypertrophy in the late activated LV free wall. The increase in regional myocardial mass results from myocyte hypertrophy. In both hypertrophying and normal hearts pacing is not associated with a disproportional increase in myocardial collagen. In normal hearts capillary density is lower in the hypertrophied than in the non-hypertrophied regions. The lower capillary density is also observed in globally hypertrophic hearts due to pressure overload.

\section{Load regulated myocardial growth}

Asynchronous electrical activation causes regional differences in workload, which is lower in early- than in 
late-activated myocardial regions. ${ }^{23}$ Therefore, the findings of the present thesis corroborate the hypothesis that myocardial structure adapts locally to local mechanical load. This hypothesis is based on several observations. The possibility to regulate myocyte growth at the local level was demonstrated in a mathematical model of LV adaptation to hemodynamic changes, ${ }^{4}$ In an in vivo study Cooper et al..$^{5}$ showed that regional unloading of a papillary muscle in an intact right ventricle is associated with atrophy of the unloaded myocardial region. In vitro studies showed that stretching of cardiomyocytes is associated with a hypertrophic response in these cells." Recently, the importance of local load in the regulation of local growth was further delineated by a study in an in situ porcine heart model. In this study stretch was applied locally to the right ventricle. After $30 \mathrm{~min}$ of stretch the immediate early genes c-fos and c-myc were upregulated, which is an early sign of the onset of a hypertrophic response. $^{8}$

Asymmetric myocardial hypertrophy, similar to the one in the present study was observed in patients with left bundle branch block. ${ }^{9}$ This disorder is associated with an electrical activation pattern similar to that during RV apex pacing. In these patients the early activated septum was found to be thinner than the late activated posterior wall." The similarity between the findings of asymmetric hypertrophy induced by ventricular pacing and those induced by left bundle branch block support the idea that asymmetric hypertrophy is caused by asynchronous electrical activation of any kind, leading to differences in workload of myocardial regions.

During ventricular pacing opposite growth patterns are observed in early and late activated regions and the growth response differs between hypertrophying and nonhypertrophic hearts. In the chronically paced hypertrophying hearts there is no excess hypertrophy in the late activated myocardial areas, as is the case in the nonhypertrophic hearts. This finding may indicate that hearts. with pre-existent hypertrophy are less susceptible to extra mechanical stimulation. This idea is supported by the experiments of Schunkert et al. In a study on isolated rat hearts these investigators found a reduced growth-response of hypertrophic myocardium to mechanical stimulation. ${ }^{10}$

\section{Distribution of myocardial blood flow during chronic pacing}

The present study supports previous findings that during short-term pacing myocardial blood flow decreases 
in regions located close to the pacing site as compared with sinus rhythm, thus resulting in non-uniform distribution of blood flow during pacing. ${ }^{23}$ This initially nonuniform distribution of blood flow disappears after 6 months of ventricular pacing. Because the distribution of blood flow is proportional to workload, the distribution of local workload becomes probably more uniform during long-term than during acute ventricular pacing. This greater uniformity on the long run is most likely caused by the development of local septal hypertrophy, decreasing workload per unit volume of the muscle to the level of that in early activated regions. These findings support the notion that hypertrophy, at least initially, is a functional process to normalize load per unit volume of muscle.

Despite this normal flow density, it can not be excluded that the hypertrophied regions are more susceptible to ischemia, because of the increased diffusion distance due to myocyte fiber thickening. In globally hypertrophied ventricles due to pressure overload, after all, the decreased capillary density is associated with a higher susceptibility to ischemia under more compromising hemodynamic circumstances." Decreased capillary density and increased diffusion distances have also been reported for the hypertrophied, non-infarcted region of hearts with coronary ligation. ${ }^{12}$
In a study on myocardial perfusion and function in pacemaker patients, Tse et al. ${ }^{13}$ found that myocardial perfusion deteriorates between 2 and 4 years of pacing. This change was also associated with a reduction in ejection fraction. Such a reduction in myocardial blood flow and ejection fraction was not observed within the 6 months experimental period in the present canine studies. This may indicate that either the longer duration and/or certain patient related factors underlie the aberrations observed by Tse et al. ${ }^{13}$

\section{Activity of metabolic enzymes}

In chronically paced asymmetrically hypertrophied left ventricles the metabolic enzyme activity was found to be uniformly distributed. This, combined with the observation that myocardial blood flow per gram tissue is also uniformly distributed, indicates that myocardial metabolism is distributed uniformly in these hearts. Compared to SHAM animals, however, the activities of such enzymes as citrate synthase and fructose-6phosphate kinase decreased similarly in the various regions of the chronically paced left ventricles with asymmetric hypertrophy. These findings do not corroborate our initial working hypothesis that in the asym- 
metrically hypertrophied hearts enzyme activities would be nonuniformly distributed. The absence of any regional difference in enzyme activities in chronically paced hearts indicates that changes in myocardial enzyme activity are not related to the degree of hypertrophy. The mechanism responsible for the globally reduced enzyme activity in paced hearts is subject to further investigation.

\section{Does chronic pacing lead to heart failure?}

The present study shows that ventricular pacing reduces acutely LV function in both normal and hypertrophic hearts. The reduction in LV function induced by ventricular pacing, however, is similar after 15 minutes and 6 months of pacing, indicating that, although acute pacing decreases cardiac function acutely, the myocardium is able to adapt in such a manner that cardiac function is maintained during long-term pacing. In normal canine hearts the adaptation to long term pacing consists of global LV hypertrophy (increase in LV/body weight ratio by $25 \%$ and dilation of the LV cavity by $18 \%$ ). Such a perturbation in cardiac geometry is reached after only 6 months of pacing. Because hypertrophy in general is a risk factor for heart failure and myocardial enzyme activities were found to be reduced to a similar extent in paced and globally hypertrophic hearts, it can not be excluded that during pacing of longer duration cardiac function will be further impaired. Findings in a large prospective patient study are in support of this notion. In an 8-year follow-up study of 225 patients with sick sinus syndrome, single chamber atrial pacing was compared to single chamber ventricular pacing. Atrial pacing was associated with a significantly higher survival rate, fewer thromboembolic complications, less heart failure and a decreased consumption of diuretics. ${ }^{1}$ Moreover, ventricularly paced patients showed a decreased LV fractional shortening and an increased dilation of the left atrium. ${ }^{\text {15 }}$ Interestingly, these changes were not statistically significant after a mean followup of 3.3 years. ${ }^{10}$ The latter finding indicates that transition to heart failure only occurs many years after onset of ventricular pacing, which may explain the absence of an additional reduction of cardiac function after 6 months of pacing in our experiments. The observations in these studies indicate that it is worthwhile to look for pacing sites which cause less asynchronous activation and/or reduction in pump function.

\section{Can pacing be more physiologic?}

In a first attempt to approximate physiological 
pacing, various pacing sites and combinations of pacing sites were compared with respect to their acute hemodynamic effect (chapter 8): An interesting observation in this study on normal hearts was that in multisite pacing cardiac function poorly correlates with QRS duration, despite the fact that QRS duration is shortened significantly, In normal hearts, optimal cardiac function could be obtained by pacing from one single site (i.e. LV apex) despite a slightly longer QRS duration than during RV apex pacing. These findings indicate that the sequence of electrical activation is a more important determinant of cardiac function than the synchrony of activation.

\section{Pacing in hypertrophic obstructive cardiomyopathy}

In hypertrophying hearts pacing at the RV apex suppresses hypertrophy selectively in the earliest activated septal regions. These findings indicate that asynchronous electrical activation can induce remodeling even in hypertrophic hearts. Furthermore, these findings may explain part of the beneficial effects of ventricular pacing in patients with hypertrophic obstructive cardiomyopathy (HOCM). In these patients pacing at the RV apex causes an acute hemodynamic improvement, followed by a progressive improvement during at least the first year of pacing. Because in patients part of the improvement is still evident after switching off the pacemaker, remodeling has been proposed as mechanism to explain these findings.

The suppression of hypertrophy in the apical septum, as obtained by RV apex pacing, is less relevant for HOCM patients, because in these patients usually the basal septum shows the most pronounced thickening. One may argue that a pacing site in the septum closer to the base of the LV may be preferable to RV apex pacing. This pacing site, however, has been avoided since Gadler et al. ${ }^{\prime \prime}$ showed that pacing from this site does not lead to acute hemodynamic improvement. These investigators chose a high septal pacing site to induce minimal asynchrony of activation. This thesis, however, shows that asynchronous activation is required to obtain local remodeling. Therefore, it seems worthwhile to search in HOCM patients for a pacing site, which induces asynchronous activation and is high enough in the septum to cause selective thinning of the basal septum. This, however, will potentially be the cost of the acute decrease in LV-aortic pressure gradient. It may quite well be that high septal pacing is less effective in patients with severe HOCM, but that high septal pacing has to be started early in the course of the disease, when LV outflow tract obstruction is minor, to reduce progression of the disease. 
The chronic pace model to study myocardial adaptation in vivo

Recently the mechanical loading conditions during pacing have been quantified in great detail in studies employing MRI tagging. ${ }^{10}$ The present thesis further characterizes the chronic effects of ventricular pacing.

Chronic pacing may be a suitable model to study load regulated growth locally, and to uncouple local load from systemically acting factors, because during ventricular pacing all regions of the myocardium are subjected to the same level of circulating factors with potentially growth promoting activity, like noradrenaline and angiotensin. Therefore, the effects of local load and more globally acting factors can be distinguished.

\section{Myocardial blood flow determination with fluorescent microspheres}

Microspheres lodging in the microcirculation are widely used in experimental research to obtain information about regional perfusion within organs. Microspheres labeled with radioactive isotopes are considered to be the 'gold standard' for blood flow measurements in an experimental setting. The use of radioactivity, however, has serious drawbacks, especially in chronic experiments: special animal housing is needed and, more importantly, with longer duration radioactivity of the unstable isotopes may decline to undetectable levels. As an alternative for radioactively labeled microspheres (RM) Glenny and others validated the use of fluorescent microspheres (FM) ${ }^{1020}$ In their method negative pressure filtration of the digested tissue was used to recover the microspheres from the samples. This filtration method, however, is laborious, relatively expensive and has potential pitfalls inherent to transfer of the filter from one tube to another. Therefore, a different approach to extract FM from the tissue was developed, i.e. extraction by sedimentation. It was shown that with the use of the sedimentation method blood flow could be determined as accurate as with RM in normal and ischemic myocardium, and in various other organs. Furthermore, compared with the filtration method ${ }^{19}$ a reduction in time and costs was achieved.

To find out whether uniformity of myocardial blood flow is affected during chronic pacing, the FM method was validated in chronic experiments. FM proved to be superior to RM in chronic experiments. The reliability of the RM method decreased over time due to leaking of label from the beads. Therefore, the variability in blood flow as assessed with RM increased over time and blood 
flow was systematically underestimated with some of the radioactive labels used. These findings are surprising considering the fact that RM have been used for chronic experiments for more than 20 years. ${ }^{2124}$

\section{Fluorescent microsphere technique}

Using the sedimentation method saves time and money, compared with the filtration method. Nevertheless the time needed for sample processing and determination of fluorescence is still substantially longer than in the RM method. This disadvantage still discourages investigators to switch from RM to FM, especially when the number of samples is large. Therefore, automation of the FM method is an important issue for the near future.

The FM method consists of two major steps: the recovery of microspheres (sample processing) and the time needed for fluorimetry. Several options are available to shorten the latter step. With a wellplate reader the fluorescence in 96 microtiter wells can be read in an automated fashion, significantly reducing the user time for fluorimetry. This method, however, has been shown to be less reliable than cuvette reading and is therefore not recommended. ${ }^{19}$ Another option to reduce time for fluorimetry may be the use of an autosanpler in combination with a fluorimeter. A potential problem in this approach is the larger sample volumes required to avoid carry-over, which increases the risk of introducing air bubbles and, thus, inaccurate measurements. When these potential problems are solved, the autosampler may be an asset, because it will substantially reduce time for fluorimetry. Most of the total time per measurement, however, is spent on sample processing.

Recently a modular robot system has been introduced that might be a breakthrough in FM technology. ${ }^{23}$ In this robot system digestion in specially designed filtration tubes is followed by negative pressure filtration for microsphere isolation, while fluorescence extraction and fluorimetry are performed automatically. This system, however, requires large investments (in the order of \$200.000) while the filtration devices (one needed per sample, for one-time use only) are expensive (about $\$ 2$ a piece).

Another method used is quantification of FM in dissolved tissue by means of a fluorescence activated cell sorter (FACS). ${ }^{20.26}$ The advantage of the FACS method is that the entire sample is analyzed which could save time because solved tissue samples do not have to be filtered. With the development of tailor made counters for FM as a cheaper alternative to a FACS analyzer, ${ }^{20}$ this method is 
probably the most promising.

Alternatively, regional blood flow can be determined by counting FM in tissue sections. A microscope coupled to a video camera with up to five fluorescence filters detects the location of each bead in each slice, yielding a 3D localization of all microspheres. ${ }^{*}$ Combination with a fully automated cryomicrotome may provide a time saving and highly accurate way to determine regional myocardial blood flow.

\section{CONCLUSIONS}

- In the LV wail local cardiac load is an important regulator of local cardiac growth.

- In normal hearts chronic asynchronous electrical activation, as induced by ventricular pacing, leads to asymmetric hypertrophy, i.e. reduced wall thickness in early and increased wall thickness in late activated regions. In these hearts the increased wall thickness in late activated regions is associated with myocyte hypertrophy.

- In hypertrophying hearts due to pressure overload chronic asynchronous electrical activation leads to a suppression of hypertrophy development in early activated myocardium, which is associated with reduced myocyte thickness.

- The distribution of myocardial blood flow within the LV wall becomes nonuniform during acute pacing, i.e. a decreased blood flow in the early and increased blood flow in the late activated regions. During chronic pacing blood flow (per unit volume) becomes uniform again. Assuming that the distribution of blood flow is proportional to workload this finding indicates that local growth regulation can normalize workload per unit of tissue volume.

- In chronically paced hearts the activities of metabolic enzymes, like citrate synthase and fructose-6-phosphate kinase, are not locally different but are reduced throughout the LV wall, similar to those in left ventricular hypertrophy owing to pressure overload. This indicates that enzyme activities are not directly related to the degree of hypertrophy but rather to some systemic factor(s),

- Ventricular pacing reduces heart function acutely, but does not further reduce it within 6 months of pacing. Unlike in hypertrophying hearts in normal hearts chronic ventricular pacing is associated with increased LV wall mass and LV cavity volume.

- The site of pacing, and therefore the sequence of 
electrical activation, is a more important determinant of cardiac function than the synchrony of electrical activation. The LV apex appears to be a favorable pacing site.

- The fluorescent microsphere method becomes more attractive when the centrifugal sedimentation method is used for microsphere isolation from the tissues.

- Fluorescent microspheres are superior to radioactive microspheres for the determination of myocardial blood flow in chronic experiments.

\section{LITERATURE}

1. Kusumoto FM, Goldschlager N. Medical progress: cardiac pacing. N Engl J Med. 1996:334:89-?

2. Prinzen FW, Augustijn CH, Arts T, Allessie MA, Reneman RS. Redistribution of myocardial fiber strain and biood flow by asynchronous activation. Am j Physiol. 1990;259: 11300-11308.

3. Delhaas $T$, Arts T, Frinzen FW, Reneman RS. Regional fibre stress-fibre strain area as an estimate of regional blood flow and oxygen demand in the canine heart. J Physiol (London) 1994:477.3:481-496.

4. Arts T, Prinzen FW, Snoeckx LHEH, Rijcken JM, Reneman RS, Adaptation of candiac structure by mechanical feedback in the environment of the cell: A model study. Biophysical 1. 1994;66:953-961.

5. Cooper IV G. Cardiocyte adaptation to chronically altered load. Ann Rev Physiol $1987 ; 49: 501-518$.

6. Sadoshima J, Izumo S. The cellular and molecular response of cardiac myocytes to mechanical stress. Annu Rey Physiol 1997:59:551-571.

7. Sadoshima J, Xu Y, Slayter HS, Izumo S. Autocrine release of angiotensin II mediates stretch-induced hypertrophy of cardiac myocytes in vitro. Cell. $1993,75 \cdot 977-984$

8. Meghii P, Nazir SA. Dick DI, Baily MES, Johnson KI. Lab MI. Regional workload induced changes in electrophysiology and immediate early gene expression in intact in situ porcine heart. J Mol Cell Cardiol. 1997:29:3147-3155.

9. Prinzen FW, Cheriex EC, Delhaas T, Van Oosterhout MFM, Arts T, Wellens HIJ Reneman RS. Asymmetric thickness of the left ventricular wall resulting from asynchronous electric activation: A study in dogs with ventricular pacing and in patients with left bundle branch block. Am Heart J. 1995:130:1045-1053

10. Schunkert H. Weinberg EO, Bruckschlegel G, Riegger Al, Lorell BH. Alteration of growth response in established cardiac pressure overload hypertrophy in rats with aortic banding. I Clin Invest 1995,96-2768-2074

11. Snoeckx L.HE.H, Van der Vusse G], Van der Veen FH, Coumans WA, Reneman RS. Recovery of hypertrophied rat hearts after global ischemia and reperfusion at different perfusion pressures. Eur I Physiol (Pfluegers Arch). 1989-413:303-312.

12. Anversa P. Beghi C. Kilkkawa Y. Olivetti G. Myocardial infarction in rats. Cinc Res. 1986,58:26-37.

13. The H-F, Lau C.P. Long-term effect of right ventricular pacing on myocardial perfusion and function. I Am Coll Cardiol 1997/29:744-749

14. Andersen HR, Nielsen JC, Thomsen PEB, Thuesen L, Mortensen PT, Vesterlund T Pedersen AK. Long-term follow-up of patients from a randomised trial of atrial versus ventricular pacing for sick-kinus syndrome. Lancet. 1997:350:1210-1216

15. Nielsen IC, Andersen HR. Thomsen PEB. Thuesen L. Mortensen PT. Vesterlund T. Pedersen AK. Heart failure and echocardiographic changes during long-term follow-up of patients with sick sinus syndrome randomized to single-chamber atrial or ventricular pacing. Circulation. 1998-97:-987-995.

16. Faenestrand S, Ohm O-J. A. time-related study of the hemodynamic benefit of atrioventricular synchronous pacing evaluated by doppler echocandiography. PACE 1985;8:838-848:

17. Gadler F, Linde C, Juhlin-Dannfeldt A, Ribeiro A, Ryden L. Influence of right ventricular pacing site on left ventricular outflowtract obstruction in patients with hypertrophic obstructive cardiomyopathy. I Am Coll Cardiol. 1996;27:1219-1224.

18. Prinzen FW. Hunter WC. Wyman BT McVeigh ER. Mapping of regional myocardial strain and work during ventricular pacing: experimental study using MR! tagging. JACC, in press. 1999;

19. Glenny RW, Bernard S, Brinkley M. Vatidation of fluorescent-labeled microspheres for measurement of regional organ perfusion. I Appl Physiol. 1993;74:2585-2597.

20. Austin GE, Martino-Salzman D, Justicz AG, Brooks AC. Tuvlin MB, Hunter RI Thompson NK. Determination of regional myocardial blood flow using: fluorescent microspheres. Am / Candiovasc Pathol. 1993:4:352-357.

21. Kersten IR. Pagel PS, Warltier DC. Protamine inhibits coronary collateral developments in a canine model of repetitive coronary occlusion. Am ] Physiol. 1995;268: $\mathrm{H} 720-\mathrm{H} 728$.

22. Grover GI Houghton IM Weis HR Propranotol and thyroxine-induced hypertrophic rabbit hearts: effect on heart size and regional $\mathrm{O}_{2}$. supply/consumption variables. Basic Res Cardiol. 1988;83:268-276.

23. Symons ID, Pitsillides KF, Longhurst IC Chronic reduction of myocardial ischemia does not attenuate cononary collateral development in miniswine Circulation. 1992;86:660-671.

24. Youngelman DF, Kahng KU, Rosen BD, Dresner LS, Wait RB. Effects of chronic cyclosporine administration on renal blood flow and intrarenal blood flow dirtribution. Transplantation. 1991;51:503-509. 
55. Raab 5, Thein E, Harris AG, Kleen M. Hicer O Mesmer K Vatidation of an auteasurement of regional organ operfusion. ASEB J 1998;12:A390

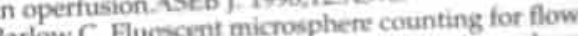
26. Rorvick L., Kelly J, Glentry Rarlow measurements. Proceedin.

methods, Seattle, 1998. 27. Bernand SL Glenny RW, Polissar NL., Lucht DL Lakstmmensured by fluorescent of pulmonary and bronchial blood supply

microspheres. I Appl Physiol. 1996;80:430- felly I Hetrrogeneity of flow and

28. Ewen I, Bariow C, Vaupel J. Glenny R, elly I. Procesdings of the 3rd int metabolism in rat heart during graded inemia. Procerding. 1998.

conference of fluorescent microsphere methis, Seattic, 1978 .

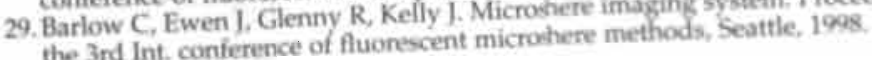




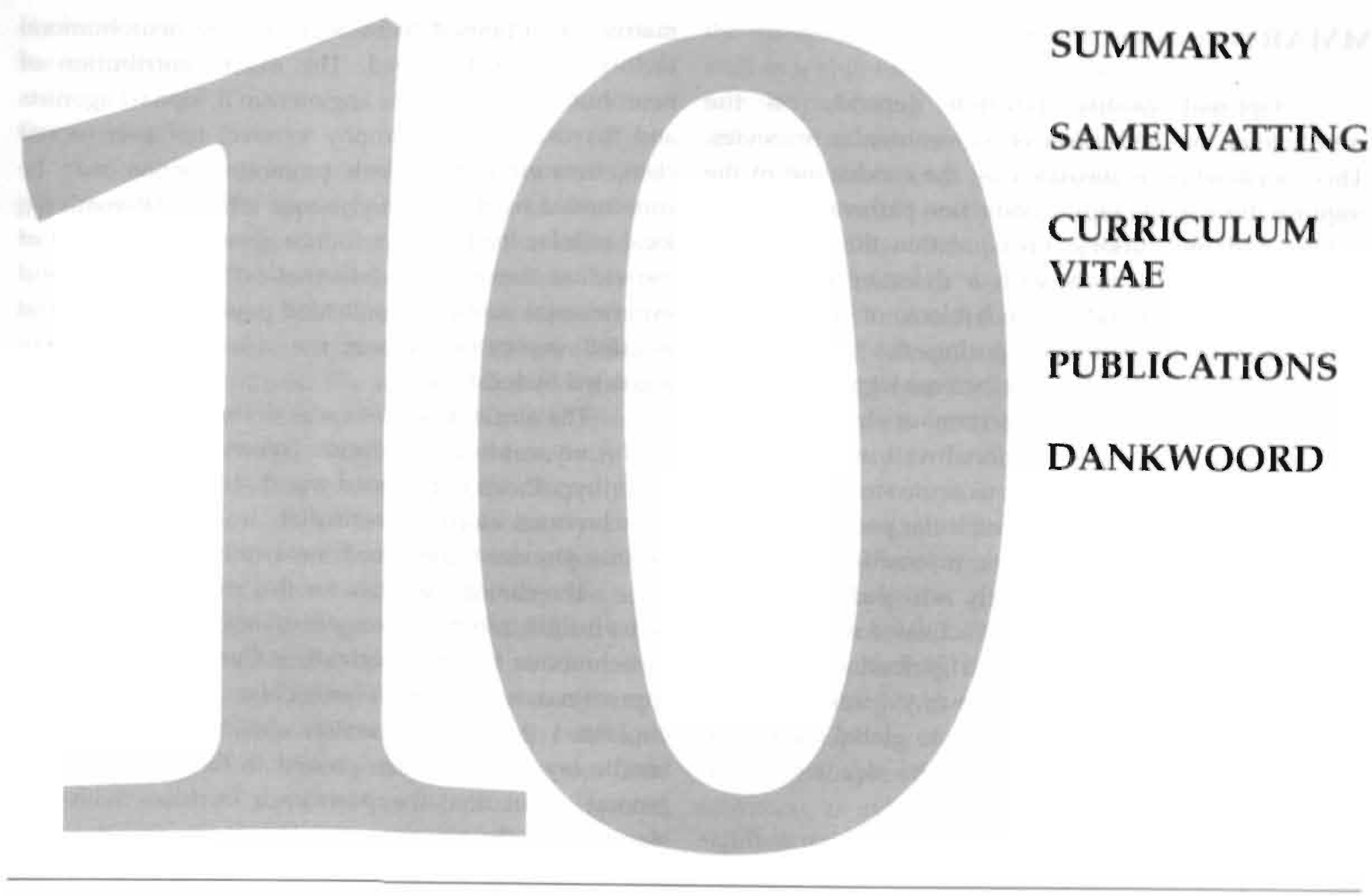




\section{SUMMARY}

Optimal cardiac function depends on the coordination of contraction of all ventricular myocytes, This coordination is mediated by the conduction of the impulse through the rapid conduction pathways (the His bundle and the Purkinje fibers) within the ventricular myocardium. In patients with a defective conduction system, as in left bundle branch block, or in disturbed conduction, as in ventricular pacing, the time for total ventricular depolarization may increase significantly. It is well known that abnormal asynchronous electrical activa-tion has a great impact on regional wall motion and on global pump function. In previous acute studies from this laboratory it was shown that ventricular pacing decreases fiber shortening, contractile work, myocardial blood flow and oxygen consumption in early activated regions and increases these parameters in late activated regions.

The consequences of this redistribution of myocardial workload on the long run are as yet unknown. The ventricular wall is known to adapt to global changes in workload by changing cardiomyocyte size and extracellular matrix composition.

Changes in cardiomyocyte size and extracellular matrix are supposed to be regulated by neurohumoral factors and cardiac load. The exact contribution of neurohumoral factors, like angiotensin II, alpha-1 agonists and thyroxin, to hypertrophy in vivo, however, is not clear, because their growth promoting action may be confounded by their hemodynamic effects. Alternatively, local cellular loading may induce growth at the level of individual myocytes. Mathematical simulations and experimental studies on unloaded papillary muscles and isolated myocytes support the role of local growth regulated by local load.

The aim of this thesis was to study the adaptation of the myocardium in chronic ventricular pacing. The major hypothesis to be tested was that in the in situ heart asynchronous electrical activation leads to asymmetric hypertrophy due to a non-uniform distribution of workload.

The clinical rationale for this study was to obtain better insight into the etiology of myocardial adaptation in asynchronous electrical activation. Currently, worldwide approximately 400.000 ventricular pacemakers are implanted yearly. Conduction abnormalities, like left bundle branch block, are present in $0.02-0.3 \%$ of the general population, the prevalence increases with age. Nevertheless, the consequences of long term asynchronous 
electrical activation for myocardial function and structure are not well-known.

\section{Overview of the thesis}

Because the occurrence of hypertrophy was anticipated and myocardial hypertrophy is often associated with myocardial underperfusion, first a method was developed to measure blood flow in chronic animal studies. Conventionally, blood flow measurements in the experimental setting are performed by means of microspheres labeled with radioactive isotopes. The use of radioactivity, however, has serious drawbacks: the special animal housing that is required, personel is exposed to radioactivity and the radioactive label shows decay over time. Recently, the use of microspheres labeled with fluorescent dyes was introduced as an alternative. In chapter $\mathbf{3}$ a novel method for tissue digestion and microsphere recovery, the sedimentation method, is described and validated for the use in normal and ischemic myocardium and various other organs in dogs. In anesthetized dogs with coronary artery stenosis up to six different fluorescently and five different radioactively labeled microspheres were injected. Two fluorescent and two radioactive labels were injected simultaneously to enable determination of bias between the fluorescent and radioactive microsphere methods as well as within each of the methods. Using the developed sample processing method nearly $100 \%$ of the microspheres was recovered. Blood flow values obtained by means of fluorescent microspheres corresponded very well with those obtained with the use of radioactive microspheres. The bias within the fluorescent and radioactive microsphere methods was equal small. In addition the centrifugal sedimentation method was faster and cheaper than previously described isolation procedures for fluorescent microspheres. In chapter 4 the fluorescent microsphere technique is validated for the use in chronic experi-ments. Accuracy of the fluorescent microsphere method did not decrease over time. In contrast, differences in blood flow values obtained with simultaneously injected radioactive microspheres increased when the time between microsphere injection and sacrifice of the animal was longer than one week. The error varied per isotope and per organ, indicating that labels leack from the beads. Therefore, in long-term experiments (weeks to months) fluorescent microspheres are superior to radioactive microspheres.

In chapter $\mathbf{5}$ the effects of chronic asynchronous activation, as induced by left ventricular (LV) pacing, on regional geometry and microscopic structure of the LV 
wall and on global ventricular geometry and performance were studied. Eight adult dogs were paced at physiological heart rate and with short AV interval from the LV free wall for six months. Five dogs were sham operated and served as controls. LV geometry (regional wall thickness, wall sector volume and global LV wall and cavity volume) was studied by means of 2D-echocardiography and LV function by means of pressure-volume analysis. Post mortem myocyte diameter and collagen fraction were determined by means of histological techniques.

Ventricular pacing more than doubled the QRS duration acutely. After six months of pacing, the QRS duration further increased by $20 \%$ of the value after 15 minutes of pacing. Within 6 months of pacing LV cavity and wall volume became $27 \%$ and $15 \%$ larger, respectively. The early activated LV free wall became $17 \%$ thinner, while the late activated septum became $23 \%$ thicker. Wall sector volume did not change in the LV free wall, but became $39 \%$ larger in the septum. Accordingly myocyte diameter in the septum was $18 \%$ larger than in the LV free wall. Myocardial collagen fraction was unchanged in both areas. LV pressure-volume analysis showed that ventricular pacing reduced LV function to a similar extent after $15 \mathrm{~min}$ and 6 months of pacing. From this study it is concluded that asynchronous activation induces asymmetric hypertrophy and LV dilatation. Cardiac pump function is not affected by the adaptational processes. These data indicate that local cardiac load regulates local cardiac mass of both myocytes and collagen.

Ventricular hypertrophy is frequently associated with diminished perfusion and altered metabolism. In chapter $\mathbf{6}$ it was investigated whether local differences in the degree of hypertrophy within the same heart were associated with local differences in myocardial perfusion and in the activity of various metabolic enzymes. Measurements were performed in the same animals as presented in chapter 5. An other group of dogs $(n=4)$ with hypertrophied hearts due to pressure overload was used as positive control.

Histological studies showed that the number of capillaries per myocyte was not different between early and late activated regions in the paced hearts and was similar in these hearts and the globally hypertrophied hearts. Absolute values of myocardial blood flow, determined by means of fluorescent microspheres, did not change during the 6 months pacing period. The uniformity of regional blood flow, defined as the ratio of myocardial blood flow in LV free wall and septum, however, changed. The LV 
free wall/septum blood flow ratio was close to one during baseline sinus rhythm, decreased to 0.7 after 15 minutes of ventricular pacing and returned to 0.9 after 6 months of ventricular pacing. Because the distribution of myocardial blood flow is closely related to the distribution of regional work the more uniform myocardial blood flow after chronic ventricular pacing suggests that local hypertrophy normalizes workload per unit of tissue volume.

In both the hearts with pressure overload hypertrophy and in the paced hearts myocardial tissue activities of fructose-6-phosphate kinase and citrate synthase were lower than in control hearts. In paced hearts the reduction in the activities of these enzymes were not limited to the hypertrophied regions, but were markedly lower $(52 \%$ and $32 \%$, respectively) throughout these hearts. These data indicate that activity of these enzymes is determined by systemic rather than local factors.

While in chapter 5 it was shown that in normal, non-hypertrophic hearts ventricular pacing ultimately leads to reduced wall thickness in early- and increased wall thickness in late-activated regions, in chapter 7 it was investigated whether ventricular pacing can induce similar structural adaptations in hypertrophying hearts. LV hypertrophy wasi induced by coarctation of the ascen- ding aorta in puppies. During the progression of hypertrophy, seven animals were paced for six months from the conventionally used right ventricular apex (the $\mathrm{POH}$-pace group), while five banded but non-paced dogs served as control (POH-control), 2D-echocardiography and X-ray marker detection were used to measure global LV wall volume and the relative changes in volume of $5 \mathrm{LV}$ wall sectors (apical and basal septum and anterior, posterior and lateral LV wall). In the POH-control group LV wall volume increased, varying from $27 \%$ in the apical septum to $30 \%$ in the lateral LV wall. In the POH-pace group the increase in regional wall volume in 4 out of 5 sectors ranged from 31 to $35 \%$. In the early-activated apical septum, however, this increase was significantly smaller $(17 \%)$. In these hearts myocyte diameter was smaller in the apical septum than in the lateral LV wall. Chronic pacing did not further deteriorate the reduced LV function seen after acute pacing. It is concluded that in hypertrophying hearts chronic pacing at the right ventricular apex suppresses the development of hypertrophy in the early-activated apical septum. In contrast to normal hearts ventricular pacing does not lead to excess of hypertrophy in lateactivated regions.

After having demonstrated that chronic asynchro- 
nous electrical activation has potentially unfavorable effects on LV function in chapter 8 it was investigated whether hemodynamic improvements can be obtained by using other pacing sites than the conventional right ventricular apex. Also the hemodynamic effect of multisite pacing was studied in short-term studies in dogs. Two strategies to optimize LV function in ventricular pacing were compared: minimal asynchrony and optimal sequence of electrical activation. ECG and hemodynamics (aortic flow, cardiac output, LV pressure and its maximal rate of rise ( $\mathrm{LVdP} / \mathrm{dtpos})$ and fall $(\mathrm{LVdP} / \mathrm{d}$ tneg) were measured in anesthetized open-chest dogs $(\mathrm{n}=7)$ with normal hearts. QRS duration was $47 \mathrm{~ms}$ during sinus rhythm and increased to $110 \mathrm{~ms}$ during atrium sensed ventricular pacing at the right ventricular apex with short AV-interval. When pacing at the LV apex and LV base QRS duration increased only by $8 \%$ and $15 \%$, respectively, as compared with RV apex pacing. Stroke volume, LVdP/dtpos and $\mathrm{LVdP} / \mathrm{dtneg}$, however, were larger during LV apex (15\%, $10 \%$ and $15 \%$, respectively) and LV base pacing (11\%, 3\% and $3 \%$, respectively) than during right ventricular apex pacing. Systolic LV pressure was not influenced significantly by the site of pacing. Biventricular pacing (right ventricular apex together with one or two LV sites) decreased QRS curation by approximately 20\% as compared with right ventricular apex pacing alone but did not improvi stroke volume, LVdP/dtpos and $\mathrm{LVdP} /$ dtneg beyord those during pacing at the LV apex alone. It is concludid that the sequence of electrical activation is a more impotant determinant of ventricular function than the synchrony of activation. For optimal LV function the selection of anoptimal single pacing site, like the LV apex, is more impotant than pacing from multiple sites. 


\section{SAMENVATTING}

Het hart is de stuwende kracht van de bloedsomloop, een goede hartfunctie is een absolute voorwaarde voor het goed functioneren van de overige organen. Dat het hart normaal gesproken ongeveer zestig keer per minuut klopt komt door de vorming van spontane electrische impulsen in de sinusknoop, gelegen in de rechter boezem van het hart (voor een schematische voorstelling van het hart zie figuur 2.2). Bij slecht functioneren van de sinusknoop klopt het hart te langzaam, met als gevolg dat men zich moe voelt, duizelingen heeft of flauw valt. Ook andere hartziekten kunnen een traag hartritme veroorzaken. De normale hartfrequentie kan hersteld worden met behulp van een pacemaker ('gangmaker'). Een moderne pacemaker is klein en kan, onder plaatselijke verdoving, onderhuids worden ingebracht. De pacemaker is met draden (electroden) aan het hart verbonden, die de noodzakelijke elektrische prikkel aan het hart doorgeven. Tijdens de pacemaker implantatie worden deze draden door de aders opgeschoven en in de rechter boezem en/of rechter hartkamer gelegd. Jaarlijks worden wereldwijd 400.000 pacemakers geïmplanteerd. Een pacemaker kan weliswaar de hartfrequentie weer normaliseren, maar de manier waarop de electrische prikkel door het hart geleid wordt is abnormaal: de prikkel verspreidt zich langzaam vanaf de pace-electrode in alle richtingen door de hartkamers.

De hartkamers functioneren vooral goed als het samentrekken van de verschillende gebieden van de hartspier zoveel mogelijk tegelijkertijd, synchroon, verloopt. In harten met een pacemaker verloopt het samentrekken van de hartspier niet gelijkmatig (asynchroon) vanwege de abnormale electrische prikkel geleiding. Hierdoor trekt het gebied dichtbij de plaats van de pace-elektrode vroeg in de hartcyclus samen, en de verder af gelegen gebieden later. Voorgaand onderzoek heeft aangetoond dat de mechanische belasting van vroeg samentrekkende gebieden lager en die van de laat samentrekkende gebieden hoger is dan normaal.

In dit proefschrift gaat het met name om de vraag wat de gevolgen zijn van deze abnormale manier van samentrekken op de structuur en de functie van het hart op langere termijn. Spieren passen zich aan aan de mechanische belasting: het spiergewicht neemt toe (hypertrofie) bij langdurig zware belasting en neemt af bij inactiviteit. Dit is ook bekend voor het hart: de hartspier wordt groter wanneer, bijvoorbeeld door hoge bloeddruk, de belasting 
voor het hele hart hoger is. Bij aanvang van dit onderzoek was het de verwachting dat het hart zich zou gaan aanpassen, adapteren, aan de veranderde electrische activatie op grond van de lokale verschillen in mechanische belasting.

Het is bekend dat vergroting van het hele hart kan leiden tot stoornissen in doorbloeding, stofwisseling, en pompfunctie van het hart en tot een toename van bindweefsel. Daarom is in dit proefschrift op deze aspecten extra nadruk gelegd. Om de doorbloeding van het hart in dierproeven te kunnen meten werd een bestaande methode, de microsfeer methode, aangepast. Microsferen zijn kleine bolletjes (15 micrometer, dat is ongeveer twee keer zo groot als een rode bloedcel) die worden ingespoten in de bloedsomloop. De vele (drie miljoen per keer) bolletjes worden meegenomen in het bloed en verdelen zich dan evenredig aan de doorbloeding per (stuk) weefsel. Omdat deze bolletjes vastlopen in de haarvaatjes is de hoeveelheid microsferen die na afloop van een experiment bepaald wordt een maat voor de doorbloeding op het moment van injectie. Traditioneel zijn de microsferen gemerkt met radioactieve stoffen. Hoewel dit een zeer betrouwbare methode is zijn er wel nadelen aan verbonden: radioactiviteit is schadelijk voor de gezondheid, afvoer en verwerking van het radioactief afval is kostbaar en, voor dit proefschrift belangrijk, voor lange termijn onderzoek is de methode minder geschikt vanwege verval van radioactiviteit.

Om deze redenen leek ons de fluorescerende microsfeer methode, in 1993 geïntroduceerd door Dr. Glenny (Seattle), geschikter voor het geplande onderzoek. In hoofdstuk 3 wordt een nieuwe methode beschreven voor het isoleren van microsferen uit bloed en weefselmonsters, die sneller en goedkoper is dan bestaande isolatietechnieken. Ook is de nauwkeurigheid van de gehele methode in normaal en ischemisch hartspierweefsel en in verschillende andere organen onderzocht. In honden met een vernauwing van een kransslagader werden 6 verschillende soorten fluorescerend en 5 verschillende soorten radioactief gelabelde microsferen gebruikt. Twee fluorescerende en twee radioactieve label's werden tegelijkertijd ingespoten om zowel de fout binnen elke methode als tussen beide methodes te kunnen bepalen. Met de in deze studie ontwikkelde microsfeer isolatie methode konden bijna $100 \%$ van alle microsferen herwonnen worden. De doorbloed'ings waarden verkregen met de fluorescerende microsferen kwamen goed overeen met die verkregen met de radioactieve microsferen. Beide methoden bleken even nauwkeurig. 
In hoofdstuk 4 wordt de fluorescerende microsfeer techniek gevalideerd in lange-termijn experimenten. De nauwkeurigheid van de fluorescerende microsfeer-methode bleef onverminderd hoog tijdens een test periode van maximaal twee maanden. De nauwkeurigheid van de radioactieve microsferen bleek daarentegen in de tijd sterk terug te lopen. Na een week bleken er al verschillen tussen de twee methodes te bestaan en tussen twee radioactieve tracers die gelijktijdig werden ingespoten. Deze verschillen namen toe naarmate de experimenten langer duurden. Bovendien varieerde de fout bij de radioactieve microsfeermethode per orgaan en per radioactief isotoop, hetgeen er op duidt dat radioactief label weglekt uit de microsferen. in langetermijn experimenten zijn fluorescerende microsferen dus te prefereren boven radioactieve microsferen.

In hoofdstuk 5 worden de effecten onderzocht van langdurige asynchrone electrische activatie, opgewekt met linker hartkamer pacen, op de regionale en globale geometrie (afmetingen) en microscopische structuur van de linker kamerwand en op de pompfunctie van de linker kamer. Acht volwassen honden werden gedurende 6 maanden gepaced op de vrije wand van de linker kamer. Nog eens vijf honden werden 'sham' (schijn) geopereerd en vormden de controle groep. Linker kamer geometrie (regionale wanddikte, wandsector-volume en globale linker kamerwand- en holtevolume) werd gemeten met twee dimensionale echocardiografie (2D-echo) en hartfunctie door analyse van de linker kamer druk-volumerelatie. $\mathrm{Na}$ afloop van het experiment werden myocyt (hartspiercel) diameter en de collageen (bindweefsel) fractie bepaald met histologische technieken.

Direct na starten van kamer pacen verdubbelde de duur van het QRS complex. Gedurende 6 maanden pacen nam de duur met nog eens $20 \%$ toe. De linker kamerholte en het wandvolume namen met respectievelijk $27 \%$ en $15 \%$ toe gedurende zes maanden pacen. De vroeg geactiveerde linker kamer vrije wand werd $17 \%$ dunner, terwijl het laat geactiveerde septum $23 \%$ dikker werd. In de linker kamer vrije wand bleef het wand sector volume onveranderd maar het nam met $39 \%$ toe in het septum. Myocyt diameter in het septum was $18 \%$ groter dan in de linker kamer vrije wand, een histologische bevestiging van de echografische bevindingen. Collageen fractie was onveranderd in beide gebieden. Pomp functie vermindering van de linker kamer ten gevolge van kamer pacen was hetzelfde na vijftien minuten en na zes maanden pacen. Uit deze studie kan worden geconcludeerd dat asynchrone electrische activatie leidt tot asymmetrische hypertrofie en 
vergroting van de linker kamerholte. De pompfunctie van de linker kamer wordt niet beïnvloed door deze structurele aanpassingen. Deze gegevens geven aan dat lokale belasting van de hartspier bepalend is voor de lokale massa van zowel myocyten als collageen.

Hypertrofie van de hartkamers gaat vaak samen met verminderde doorbloeding van de hartspier en een veranderd metabolisme (stofwisseling). In hoofdstuk 6 wordt onderzocht of lokale verschillen in de mate van hypertrofie binnen hetzelfde hart samen gaan met lokale verschillen in hartspier doorbloeding en in de activiteit van verschillende metabole enzymen. Deze metingen werden gedaan bij de honden die in hoofdstuk 5 werden beschreven. Een extra groep honden $(n=4)$, met hypertrofe harten ten gevolge van linker kamerdruk overbelasting, werden gebruikt als positieve controle. Met histologische technieken werd aangetoond dat het aantal capillairen per myocyt gelijk was in de wel en niet hypertrofe delen van de linker kamerwand en ook niet verschillend waren van honden met een hypertroof hart. Omdat de diameter van de myocyten in hypertrofe hartspier groter is, houdt dit in dat de diffusie afstand voor zuurstof ook groter is, en daarmee de zuurstofvoorziening moeilijker.

De hartspier doorbloeding, gemeten met fluores- cerende microsferen en uitgedrukt per gram weefsel, veranderde niet tijdens chronisch pacen. De gelijkmatigheid van de verdeling van de doorbloeding, gedefinieerd als de verhouding van de doorbloeding in linker kamer vrije wand en septum veranderde echter wel. Deze verhouding was ongeveer 1 tijdens normaal hartritme (sinus ritme), nam af tot 0.7 na vijftien minuten pacen en normaliseerde naar 0.9 na zes maanden pacen. Het is bekend dat de verdeling van de doorbloeding van de hartspier nauw gerelateerd is aan de verdeling van regionale arbeid. De gelijkmatige doorbloeding na zes maanden pacen suggereert daarom dat de lokale hypertrofie de arbeid per eenheid weefsel volume normaliseert.

De activiteit van fructose-6-fosfaat en citraat synthase, twee belangrijke enzymen van de stofwisseling in de hartspier, was respectievelijk $52 \%$ en $32 \%$ lager in de harten met druk overbelastings hypertrofie en in de gepacede harten dan in de controle harten. Deze enzymen waren niet alleen verlaagd in het hypertrofe gebied van de gepacede harten, maar ook in de andere gebieden. Deze gegevens geven aan dat de activiteit van deze enzymen vooral door systemische in plaats van lokale factoren wordt bepaald.

In hoofdstuk 5 werden de gevolgen beschreven 
van pacen in normale harten, in hoofdstuk 7 wordt onderzocht of pacen in hypertrofe harten leidt tot dezelfde structurele aanpassingen als beschreven in hoofdstuk 5 . Linker kamer hypertrofie werd opgewekt door bij jonge honden (8-10 weken oud) een bandje om de grote lichaams slagader te doen (aorta-banding). De honden groeien, het bandje groeit echter niet mee, waardoor er een vernauwing ontstaat. Hierdoor wordt de bloeddruk in de linker kamer veel hoger dan normaal en ontstaat er hypertrofie. Zeven honden werden gedurende zes maanden gepaced in een fase waarbij de hypertrofie zich nog aan het ontwikkelen was. Bij deze honden werd de punt van de rechter kamer gebruikt als paceplaats, de plaats waar ook bijna altijd voor gekozen wordt bij patiënten. De controlegroep bestond uit vier honden, die wel gebandeerd waren maar niet gepaced werden. Met echocardiografie en röntgen-marker detectie werd zowel de globale groei als de groei op vijf verschillende plaatsen van de linker kamerwand gemeten (linker kamer voor-, zij- en achterwand en basale en apicale septum). In de controlegroep varieerde de toename van het linker kamer wandvolume, van $27 \%$ in het apicale septum tot $30 \%$ in de linker kamer zijwand. In de groep honden die wel gepaced werden was er op vier van de vijf plaatsen een wandvolume toename die varieerde van $31 \%$ tot $35 \%$. Het vroeg geactiveerde apicale septum echter, nam slechts met $17 \%$ toe, significant minder dan in de andere gebieden. Daarbij was de myocyt doorsnede kleiner in het apicale septum dan in de linker kamer zijwand. Na kortdurend pacen was er een afname van de pompfunctie van het hart, welke niet verder afnam na langdurig pacen. Deze gegevens geven aan dat rechter kamer apex pacen in hypertrofiërende harten leidt tot een onderdrukking van de ontwikkeling van hypertrofie in het vroeg geactiveerde apicale septum. In tegenstelling tot normale harten ontstaat er geen extra hypertrofie in de laat geactiveerde gebieden van de linker hartkamer.

Uit de studies beschreven in de hoofdstukken 5 en 6 blijkt dat langdurig pacen en de daarmee gepaard gaande asynchrone activatie mogelijk schadelijke effecten op het hart heeft. In hoofdstuk 8 wordt daarom onderzocht of pacen op andere paceplaatsen dan de rechter kamer apex, of op meerdere paceplaatsen tegelijk, kan leiden tot een verbeterde pompfunctie. Twee manieren om de pompfunctie te verbeteren zijn vergeleken: met de ene manier wordt gestreefd naar het minimaliseren van de asynchronie, met de andere manier wordt gestreefd naar het optimaliseren van de volgorde van electrische 


\section{Chapter 10}

activatie. In 7 genarcotiseerde honden werd het electrocardiogram (hartfilmpje), het linker kamer slagvolume en bloeddruk en de snelheid van samentrekken (dP/dtpos) en ontspanning ( $\mathrm{dP} / \mathrm{dtneg}$ ) van de linker kamer gemeten. De duur van het QRS complex van het ECG (een maat voor de electische asynchronie) was $47 \mathrm{~ms}$ tijdens sinus ritme en nam toe tot $110 \mathrm{~ms}$ tijdens rechter kamer apex pacen. Vergeleken met rechter kamer apex pacen nam de QRS duur met slechts $8 \%$ en $15 \%$ toe door te pacen van respectievelijk de linker kamer apex of de basis van de linker kamer. Het slagvolume, de dP/dtpos en dP/dtneg waren echter groter tijdens LV apex (respectievelijk 15\%, $10 \%$ en 15\%) en linker kamer basis pacen (respectievelijk $11 \%, 3 \%$ en $3 \%$ ) dan tijdens pacen van de rechter kamer apex. De linker kamer bloeddruk werd niet significant beïnloed door de paceplaats. In vergelijking met pacen van de rechter kamer apex alleen was de QRS duur 20\% lager tijdens pacen van twee plaatsen tegelijk (rechter kamer apex met een of twee linker kamer plaatsen). Pacen van meerdere plaatsen leverde echter geen verbetering van de pompfunctie op ten opzichte van pacen van de linker kamer apex alleen. Deze gegevens geven aan dat de volgorde van de electrische activatie een belangrijkere factor in de pompfunctie van het hart is dan de synchroniciteit van de activatie. Een optimale functie van de linker hartkamer kan beter bereikt worden door een goede enkele pace plaats te zoeken, zoals de linker kamer apex, dan door te pacen van meerdere kanten tegelijk. 


\section{CURRICULUM VITAE}

\section{Mattheus Franciscus Maria van Oosterhout}

23 juni 1966 Geboren te Amsterdam

1978 - 1985 Montessori Lyceum Amsterdam - VWO

1985 - 1992 Studie Geneeskunde aan de Universiteit van Amsterdam

1989 Doctoraal

$1992 \quad$ Artsexamen

1989 - 1990 Propedeuse Filosofie aan de Universiteit van Amsterdam
1992

1993 - 1997 Promotie-onderzoek bij de vakgroep Fysiologie, cardiovasculair research instituut Maastricht, Universiteit Maastricht

1994 - 1996 Docent Fysiologie, Vroedvrouwenschool, Kerkrade

1997 - 1998 Assistent Kindergeneeskunde Academisch Ziekenhuis Maastricht

1998 - heden AGIO Pathologie, Academisch Ziekenhuis Utrecht 


\section{PUBLICATIONS}

\section{Full Papers}

-Prinzen FW, Cheriex EC, Delhaas T, Van Oosterhout MFM, Arts T, Wellens HJJ, Reneman RS. Asymmetric thickness of the left ventricular wall resulting from asynchronous electric activation: A study in dogs with ventricular pacing and in patients with left bundle branch block. Am Heart J. 1995;130:1045-1053.

-Van Oosterhout MFM, Willigers HMM, Reneman RS Prinzen FW. Fluorescent microspheres to measure organ perfusion: validation of a simplified sample processing technique. Am J Physiol. 1995;269:H725-H733.

- Prinzen FW, Van Oosterhout MFM, Arts T, Reneman RS. Local functional and structural changes in the myocardium during ventricular pacing. J interven Cardiol. 1996;9:319-326.

- Prinzen FW, Van Oosterhout MFM, Vanagt WYR, Storm C, Reneman RS. Optimization of ventricular function by improving the activation sequence during ventricular pacing. PACE. 1998;21:2256-2260.

-Van Oosterhout MFM, Prinzen FW, Arts T, Schreuder JS, Vanagt WYR, Cleutjens JPM, Reneman RS. Asynchrounous electrical activation induces asymmetrical hypertrophy of the left ventricular wall. Circulation. 1998;98:588-595.

-Van Oosterhout MFM, Prinzen FW, Sakurada S, Glenny RW, Hales RS. Fluorescent microspheres are superior to radioactive microspheres in chronic blood flow measurements. Am J Physiol. 1998;275:H110-H115.

-Van Oosterhout MFM, Arts T, Muijtjens AMM, Reneman RS, Prinzen FW. Ventricular pacing induces remodeling in hypertrophying dog hearts (Submitted)

- Van Oosterhout MFM, Prinzen FW, Glatz JFC, Rousseau AMJ, Vanagt WYR, Arts T, Reneman RS. Regional blood flow and activity of metabolic enzymes in canine hearts with pacing induced asymmetric hypertrophy (Submitted) 


\begin{abstract}
s
- Prinzen FW, Van Oosterhout MFM, Cheriex EC, Arts T, •Van Oosterhout MFM, Prinzen FW, Reneman RS. Wellens HJJ, Reneman RS. Chronic asynchronous Comparison of the fluorescent and radioactive electrical activation of left ventricle leads to regional microsphere methods to assess blood flow in normoxic differences in ventricular wall thickness. PACE. and ischaemic myocardium and in other organs. J Physiol. 1994;17:775. 1994;479P:125P.
\end{abstract}

- Prinzen FW, Van Oosterhout MFM, Delhaas T, Arts T, Reneman RS. Asymmetrical geometry of left ventricular wall thickness induced by chronic ventricular pacing at physiological heart rate. FASEB J. 1994;8:A794.

- Prinzen FW, Van Oosterhout MFM, Cleutjens JPM, Arts $T_{\text {t }}$ Reneman RS. Ventricular pacing leads to asymmetrical changes in ventricular wall thickness. Circulation. 1994;90:[-106.

- Van Oosterhout MFM, Prinzen FW, Reneman RS. The fluorescent microsphere method for determination of organ blood flow. FASEB J. 1994;8:A854.

-Van Oosterhout MFM, Prinzen FW, Reneman RS. The fluorescent microsphere method for determination of myocardial blood flow. Eur Heart J. 1994;15:269.

-Van Oosterhout MFM, Prinzen FW, Arts T, Reneman RS. The effects of chronic ventricular pacing on local myocardial blood flow and wall thickness. Eur J Physiol. 1995;430 (suppl):R162.

- Hales JRS, Prinzen FW, Glenny RW, Gazibarich GJ, Van Oosterhout MFM. Long term in vivo stability of radionuclide and fluorescent dye labels on microspheres used to measure blood flow. Proc Austr Physiol Pharmacol Soc. $1995 ; 26: 45 \mathrm{P}$. 
-Van Oosterhout MFM, Prinzen FW, Arts T, Reneman RS, Chronic ventricular pacing at physiological heart rate affects local myocardial blood flow and local wall thickness. JACC. 1996;27 (suppl A):344A.

-Van Oosterhout MFM, Prinzen FW, Arts T, Reneman RS. Chronic ventricular pacing induces local differences in myocardial wall thickness (WT) and changes in blood flow. FASEB J. 1996;10:A309.

- Van Oosterhout MFM, Prinzen FW, Arts T, Reneman RS. Chronic ventricular pacing affects local and global myocardial structure in dogs. Pfluegers Arch.-Eur. J. Physiol. 1996. 432:R4.

- Peschar M, Rousseau AMJ, Van Oosterhout MFM, de Groot SHM, Vos MA, Reneman RS, Snoeckx LHEH Prinzen FW. SERCA2 Protein contentin the adult canine heart hypertrophied due to pressure or volume overload. FASEB J. 1998;12:A977.
-Prinzen FW, Van Oosterhout MFM, Sakurada S, Glenny RW, Hales JRS, Arts T, Reneman RS. Fluorescent microspheres are superior to radioactive microspheres for chronic in vivo organ flow measurements. FASEB J. 1998;12:A697.

-Van Oosterhout MFM, Prinzen FW, Vanagt WYR, Arts T, Reneman RS. Chronic ventricular pacing in pressure overloaded hearts does not lead to local remodeling. J of Cardiovasc Diagn and Proced. 1998;15:PI-4, 139.

- Prinzen, FW, Van Oosterhout MFM, Vanagt WYR, Storm C, Reneman RS. Optimization of ventricular function by improving the activation sequence during ventricular pacing. PACE. 1998. 21:2256-2260.

\section{Book Chapter}

- Muijtiens AMM, Roos JMA, Van Oosterhout MFM, Prinzen FW, Egmont-Petersen M, Arts T, Reneman RS. Measuring regional hypertrophy by radiopaque myocardial markers viewed in 3-D with a single X-ray camera. In: Computers in cardiology 1997. IEEE computer society Press, Washington, 565 - 568. 


\section{DANKWOORD}

$\mathrm{Na}$ zes jaar onderzoek realiseer ik mij dat zeer velen hebben bijgedragen aan het tot stand komen van dit proefschrift. Zonder hun bijdrage zou dit boekje er niet zijn geweest en, zeker niet in de laatste plaats, zou mijn verblijf in Maastricht veel minder aangenaam zijn geweest. Al deze mensen, en met name die personen die ik niet meer bij naam zal noemen, wil ik hier bedanken voor hun bijdrage.

Frits Prinzen, mijn directe begeleider en copromotor, wil ik als eerste bedanken. Frits, vanaf het begin heb jij mij met raad en daad bijgestaan, en ingewijd in de edele (?) experimenteertechnieken. Je grenzeloze optimisme over, en het vertrouwen in het welslagen van dit werk hebben mij door mijn momenten van twijfel heen geholpen. Jouw ondersteuning die ik een jaar moest missen in verband met je Sabbatical leave (hoewel, missen.. bijna dagelijks werd er ge E-mailed) heb je tot op de laatste punt van dit proefschrift ruimschoots gecompenseerd door je hulp bij het schrijven van de teksten, discussies over het onderzoek en al het andere. Je hebt mij ook een heel andere kijk op het onderzoekers-bestaan gegeven, namelijk het congres-bezoek (liefst in verre oorden). Tijdens de vele uren vliegen en de andere (uiteraard zeer schaarse) vrije tijd tijdens die reizen was het zeer goed toeven met jou. De steeds krappere onderzoeksbudgetten noopten tot bezuinigingen op alle fronten (PeterPan Inn: uitstekende prijs/kwaliteit verhouding; twee dagen door Amerika rijden om een vliegticket uit te sparen; zo maar een greep uit de kostenbesparende maatregelen die iij wist te bedenken).

De promotoren Prof.dr. R.S. Reneman en Prof. dr.ir. T. Arts.

Rob, bedankt voor de vele inspirerende discussies gedurende de zes jaar onderzoek en de kritische pen waarmee je mijn werk gecorrigeerd hebt. Door je persoonlijke aanpak en je betrokkenheid bij mij en bij mijn werk kon ik letterlijk alles met je bespreken,

Theo, het analyseren van groei met röntgenmarkers bleek geen sinecure. In de vele gesprekken die wij daarover gevoerd hebben, heb je mij veel geleerd over analysetechnieken en beeldverwerken. De vaak mathematisch getinte onderwerpen behandelde je op een manier die zelfs voor een arts te volgen was, en met een gevoel voor humor die ik zeer waardeer. Theo, heel veel dank voor jouw bijdrage aan dit proefschrift. 
De leden van de beoordelingscommissie, Prof.dr. G.J. van der Vusse (voorzitter), Prof.dr. M.J.A.P. Daemen, Prof.dr. H.J.J. Wellens en Prof.dr. N. Westerhof wil ik bedanken voor het kritisch doorlezen van het manuscript. Also, I would like to thank Prof.dr. L. Kappenberger for reviewing the manuscript.

Het zenuwcentrum van de OK fysiologie wordt gevormd door de koffiekamer annex liever-nietrookkamer annex werkkamer van Ruud en Theo. Hier werden de operaties degelijk voorbereid ('gaarne uw operatievoorstel in drievoud inleveren') en wetenschappelijke discussies van hoog niveau gevoerd. Door het open karakter was het er altijd druk en gezellig, een plaats onontbeerlijk voor mijn onderzoek. I $\mathrm{k}$ wil iedereen bedanken die voor deze sfeer gezorgd heeft. Ruud Kruger, Theo van der Nagel en Ferenc van der Hulst wil ik bedanken voor hun onmisbare hulp bij alle experimenten.

Ruud, zonder jouw gouden handen zouden er wellicht nog meer gouden kogeltjes doelloos in de thorax rondzwerven. Ook dank voor de goede zorgen voor de honden, die in jouw dierenpension konden verblijven.

Theo, behalve je enorme technische inzicht ('...een kanaaltje even doorfluiten', 'staat er wel 'prik' op die kabel ' en 'loodschorten dragen als er met atomen gewerkt wordt'...) en chirurgische vaardigheden was je altijd bereid nog even een handje te helpen bij die oneindige hoeveelheid echo's die wekelijks gemaakt werden.

Ferenc, bedankt voor je chirurgische hulp, het zal niet eenvoudig zijn sneller door het leven te bewegen dan in de achtbaan ( 800 baan zou een betere benaming zijn) van München.

De andere medewerkers van de OK, Jet en Jo dank ik voor het 'even' tussendoor helpen. Eric van der Veen en Marc Vos wil ik bedanken voor hun interesse in ons onderzoek en het beschikbaar stellen van apparatuur.

Maaik.e, jij kwam als opvolger-AIO mij helpen met mijn laatste experimenten. Het was een vruchtbare samenwerking, voor beide partijen. Voor mij een enorme hulp om de laatste experimenten rond te krijgen, voor jou om inzicht te krijgen in hoe je de zaken zelf zou gaan aanpakken (en waarschijnlijk vooral hoe je ze níet wilde aanpakken). Heel veell dank voor je bijdrage en gezelligheid en veel succes met jouw boekje.

Ward, jij kwam ons als student assistent 'toevallen', en bleek al snel een grote aanwinst te zijn voor het onderzoek. Je hebt mij door je snelle en accurate werkwijze heel wat werk uit handen genomen. 
Jan Schreuder dank ik voor de hulp bij het 'draaien' van de PV-loops. Daarnaast heb ik in de 'world according to Jan' veel nieuwe inzichten verkregen in de medische wereld.

Cock Storm, cardioloog (Hart Centrum Rotterdam) wil ik graag bedanken voor zijn bijdrage aan onze multisite-pace experimenten.

De medewerkers van electrofysiologie, en met name Frits Schmitz, Jan Hollen en Rick Dorland will ik bedanken voor hun assistentie bij de mappingexperimenten.

Prof. dr. Allessie, dank voor het ter beschikking stellen van de mapping-apparatuur en je interesse in dit onderzoek.

Luc Snoeckx en Jan Glatz bedank ik voor hun belangstelling en bijdrage aan het onderzoek.

Het microsfeer onderzoek zou zo'n vaart niet hebben gelopen zonder de hulp van Annita Rousseau. Annita, door de hoeveelheid buisjes die jij te verwerken kreeg zal het je wel eens blue, blue-green, yellow-green en orange voor de ogen hebben gezien. Heel veel dank voor je hulp.

Henriette, jou wil ik op deze (microsfeer) plaats bedanken. We zijn zo'n beetje tegelijk bij fysiologie terechtgekomen en werden daar geconfronteerd met de microsferen van Frits. Met veel humor wist je ook de leuke kanten in te zien van het aanvankelijk mislukken van onze experimenten met onwillige filters. Bedankt voor je hulp, de gezelligheid en de vele discussies over van alles en nog wat. Ik hoop dat jij ook snel je onderzoek kunt afronden.

I would like to thank Dr. Rob Glenny and Dr Bob Hales for their invaluable help in the microsphere experiments.

Bewegende markers, van papier of van gouden bollen, hebben mij verbonden met de vakgroep Biofysica. Alle medewerkers van deze vakgroep wil ik bedanken.

Jan Corsel, veel dank voor het mij inwijden in de 'video-toren' en het aanhoren van de frustaties over het werken hiermee.

Jean Willigers, bedankt voor je hulp bij allerhande computer zaken.

De AIO's en post-docs van toen, Frank, Jons en Michaël wil ik bedanken voor hun stimulerende discussies over zinnige en minder zinnige zaken.

Jef Roos en Arno Muijtjens (Medische informatica): bedankt voor het marker-analyse werk.

Ton vd Bogaard, Huub Simons en Frans Slangen van het CPV hebben uitstekend voor de proefdieren 
gezorgd. Doordat ik wist dat de dieren goed verzorgd werden voelde ik mij minder bezwaard bij het uitvoeren van mijn experimenten. Bedankt voor het ontlasten van mijn geweten.

Mijn kamergenoten Richard, José, Michaël, en Ad en vaste kamer bezoeker Leon bedankt voor jullie - vaak luidruchtige - gezelligheid.

De secretaresses Claire, Karin en Jos wil ik bedanken voor het vinden van lege plekjes in de ruim volgeboekte agenda van Rob.

De collega's van Kindergeneeskunde te Maastricht en Pathologie te Utrecht wil ik bedanken voor hun meeleven, begrip en gezelligheid.

Lonneke Winkels (lay-out). Lon, nog voordat er een letter op papier stond had jij al aangeboden de lay-out van dit boekje op je te nemen, toen nog denkend dat well in een weekendje in elkaar te draaien. Ik vind dat dit boekje er prachtig uitziet, zonder jouw hulp was het niet meer geworden dan 'wat A4-tjes met een nietje erdoor'. Heel veel dank voor het opofferen van je vrije uren.

Paranimfen joost en Bas. Joost, ik ben blij dat je deze taak op je wilt nemen. Je hebt eigenlijk niets met het onderzoek te maken gehad, alleen van een - veel te - grote afstand de verhalen aangehoord. Heel veel dank voor je morele steun, de fietstochten, en het hardlopen. Binnenkort kunnen wij dan eindelijk die fietstocht in Frankrijk gaan maken.

Bas, jij weet inmiddels heel wat van mijn onderzoek en bent ook in die zin een waardig paranimf. Ik wil je bedanken voor je programmeer-werk en het niet aflatende ventileren van je ideeën van hoe ik het eigenlijk had moeten aanpakken (zelfs als het om leggen van parket gaat). Daarnaast ben ik je steeds meer gaan waarderen vanwege je soms eigenzinnige ideeèn, stelligheden en onze vriendschap die eruit voortvloeide.

Familie en vrienden. Ik wil een ieder van jullie bedanken voor je meeleven, voor het aanhoren van mijn verhalen en voor het niet al te boos worden als ik weer eens een balangrijke gebeurtenis miste.

Zonder mijn ouders was dit werk uiteraard nooit tot stand gekomen. Het is een oude vraag of je karakter en wat er van je terecht komt nu bepaald wordt door afkomst of door opvoeding. Voor mij is die vraag niet relevant, ik ben blij met wie ik ben en met wat ik bereikt heb, en jullie hebben daar stevig de hand in gehad. Lieve Anne en Theo, heel veel dank hiervoor. 
Lieve Pauline, het is heel geruststellend als je weet dat er een veilige thuishaven is, een gerustelling die helpt bij het doorstaan van de beproeving van het promoveren en alles wat daarbij komt kijken. Zonder jou had ik dit niet gered. Het mooiste bewijs dat ik niet als een 'nerd' zes jaar alleen maar heb 'gepromoveerd', wordt geleverd door onze twee zoons Lars en Wouter. Jongens, naast vlees snijden op zondag heb ik nu eindelijk eens tijd om met jullie te voetballen.

Truth is truth

To the end of reckoning

William Shakespeare, Measure for Measure Act V. Sc 1.

\section{Liedje}

Het duurt altijd langer dan je denkt, ook als je denkt

het zal wel langer duren dan ik denk dan duurt het toch nog langer dan je denkt.

Het is altijd veel duurder dan je denkt, ook als je denkt het zal wel duurder worden dan ik denk dan wordt het toch nog duurder dan je denkt.

Het kost meer moeite dan je denkt ook als je denkt het zal veel meer moeite kosten dan ik denk dan kost het toch meer moeite dan je denkt.

Judith Herzberg, uit Zoals 
ISBN 90-9012703 - 8

\section{Omslag Pauline M. van Oosterhout \\ Layout Lonneke Winkels \\ Drukwerk Elinkwijk BV Utrecht}

(C) 1999 Matthijs F.M. van Oasterhout, Houten, The Netherlands

All rights reserved. No part of this publication may be reproduced or transmitted in any form or by any means, electronic or mechanical, including photocopy, recording or any informationstorage or retrieval system, without permission in writing from the copyright owner. 

' 